\title{
Redox-Active Esters in Fe-catalyzed C-C Coupling
}

Fumihiko Toriyama $^{\dagger \S}$ Josep Cornella, ${ }^{\dagger \S}$ Laurin Wimmer, ${ }^{\dagger}$ Tie-Gen Chen, ${ }^{\dagger}$

Darryl D. Dixon, ${ }^{\ddagger}$ Gardner Creech, ${ }^{\ddagger}$ Phil S. Baran ${ }^{\dagger, *}$

${ }^{\dagger}$ Department of Chemistry, The Scripps Research Institute, 10550 North Torrey Pines Road, La Jolla, California 92037

${ }^{\ddagger}$ Chemical Development, Bristol-Myers Squibb,

One Squibb Drive, New Brunswick, New Jersey 08903 


\section{Table of contents}

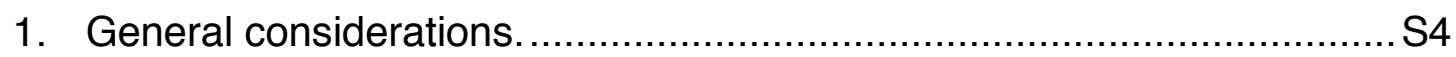

2. General Procedure A: Synthesis of NHPI and TCNHPI esters................S5

3. General Procedure for the Preparation of Diphenylzinc Reagent 2.......S13

3.1. Preparation of PhMgBr.LiCl.................................................... 13

3.2. Graphical Guide for the Preparation of PhMgBr.LiCl......................S13

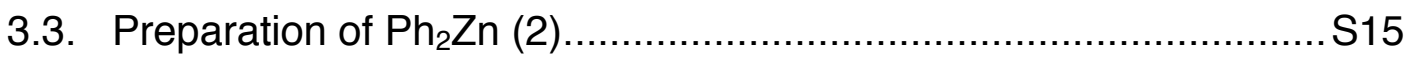

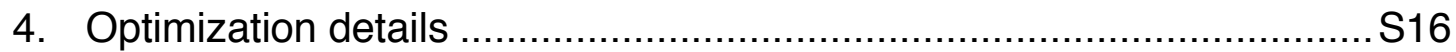

4.1. Time course of the model reaction. .............................................. 20

5. General Procedure for the Fe-catalyzed Negishi cross-coupling with redox-active esters and diarylzinc reagents (General Procedure B).

5.1. Graphical guide for the Fe-catalyzed Negishi cross-coupling with redox-active esters.

6. General Procedure for the Fe-catalyzed Negishi cross-coupling with redox-active esters generated in situ (General Procedure C).

6.1. Graphical guide for the Fe-catalyzed Negishi cross-coupling with in situ-formation of the redox-active esters.

7. General Procedure for the Fe-catalyzed Kumada-Corriu cross-coupling of redox-active esters and aryl Grignards (General Procedure D)................... S26

7.1. Graphical guide for the Fe-catalyzed Kumada-Corriu cross-coupling...

8. General Procedure for the Ni-catalyzed Negishi cross-coupling of redoxactive esters and arylzinc reagents (General Procedure E). S28

9. General Procedure for the Ni-catalyzed Negishi cross-coupling of in situ generated redox-active esters and arylzinc reagents (General Procedure F)...

10. Guide for choosing reaction conditions .......................................... $\mathrm{S} 30$

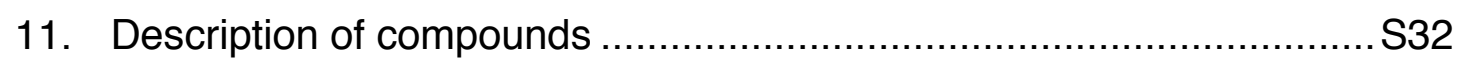

12. Procedure for a mock-medicinal chemistry gram-scale Fe-catalyzed Negishi cross-coupling with redox-active esters generated in situ................S69

12.1. Graphical Guide for the Fe-catalyzed Negishi coupling at gram scale. S70

13. Procedure for the mock-process chemistry scale-up and isolation of the Fe-catalyzed Negishi cross-coupling. S72

13.1. Graphical guide for the mock-process chemistry scale-up and isolation of the Fe-catalyzed Negishi cross-coupling......

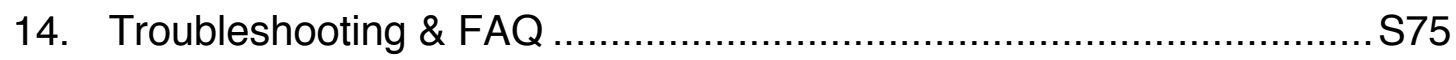

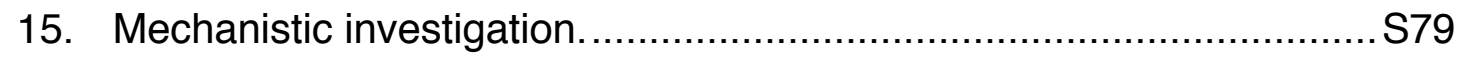

16. Iterative diarylation of cubane carboxylic acid. ................................. 880 
16.1. Graphical guide for the diarylation of cubane carboxylic acid. ......S83

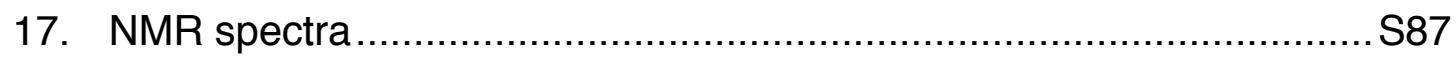

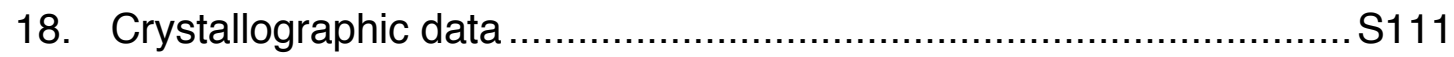




\section{General considerations.}

Tetrahydrofuran (THF), toluene (PhMe), and dichloromethane $\left(\mathrm{CH}_{2} \mathrm{Cl}_{2}\right)$ were obtained by passing the previously degassed solvents through an activated alumina column. Reagents were purchased at the highest commercial quality and used without further purification, unless otherwise stated. $\mathrm{Fe}(\mathrm{acac})_{3}$, $\mathrm{FeCl}_{3} \cdot 6 \mathrm{H}_{2} \mathrm{O}$ and $\mathrm{FeCl}_{3}$ anhydrous were purchased from Strem and Aldrich. $\mathrm{Ni}(\mathrm{acac})_{2}$ was purchased from Aldrich. Yields refer to chromatographically and spectroscopically ( ${ }^{1} \mathrm{H}$ NMR) homogeneous material, unless otherwise stated. Reactions were monitored by GC/FID, GC/MS, and thin layer chromatography (TLC). TLC was performed using $0.25 \mathrm{~mm}$ E. Merck silica plates (60F-254), using short-wave UV light as the visualizing agent or $\mathrm{I}_{2}$ or $\mathrm{KMnO}_{4}$ and heat as developing agents. NMR spectra were recorded on Bruker DRX-600, DRX500 , and AMX-400 instruments and are calibrated using residual nondeuterated solvent $\left(\mathrm{CHCl}_{3},{ }^{1} \mathrm{H}\right.$ NMR at $7.26 \mathrm{ppm},{ }^{13} \mathrm{C} \mathrm{NMR}$ at $\left.77.16 \mathrm{ppm}\right)$, TMS or hexafluorobenzene $\left({ }^{19} \mathrm{~F}\right.$ NMR at $\left.-164.9 \mathrm{ppm}\right)$ as an internal reference. The following abbreviations were used to explain multiplicities: $s=\operatorname{singlet}, d=$ doublet, $\mathrm{t}=$ triplet, $\mathrm{q}=$ quartet, $\mathrm{m}=$ multiplet, $\mathrm{br}=$ broad. Column chromatography was performed using E. Merck silica gel (60, particle size 0.043-0.063 $\mathrm{mm}$ ), and preparative TLC was performed on Merck silica plates (60F-254). High-resolution mass spectra (HRMS) were recorded on an Agilent LC/MSD TOF mass spectrometer by electrospray ionization time of flight reflectron experiments. Melting points were recorded on a Fisher-Johns 12144 melting point apparatus and are uncorrected. 
2. General Procedure A: Synthesis of NHPI and TCNHPI esters.

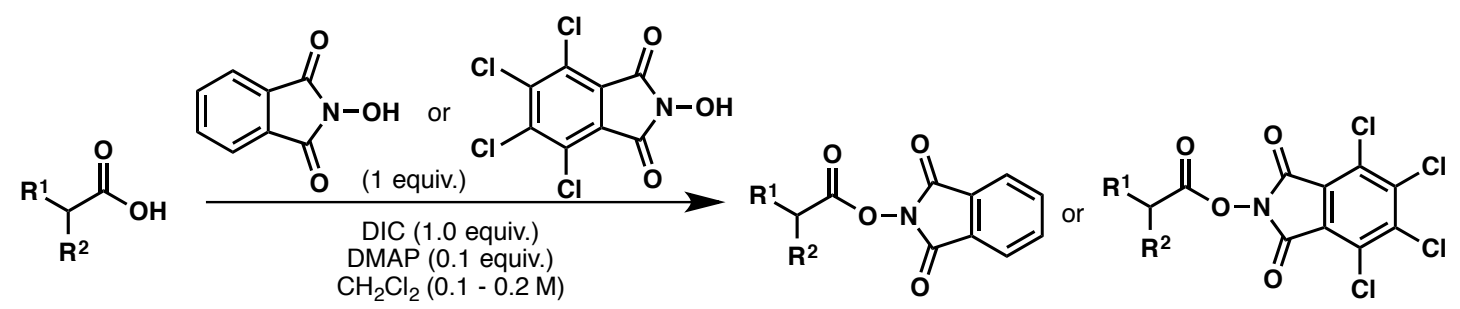

A round-bottom flask or culture tube was charged with (if solid) carboxylic acid (1.0 equiv), nucleophile ( $N$-hydroxyphthalimide, tetrachloro- $N$ hydroxyphthalimide, 1.0 equiv) and DMAP (0.1 equiv). Dichloromethane was added $(0.1-0.2 \mathrm{M})$, and the mixture was stirred vigorously. Carboxylic acid (1.0 equiv) was added via syringe (if liquid). DIC (1.1 equiv) was then added dropwise via syringe, and the mixture was allowed to stir until the acid was consumed (determined by TLC). Typical reaction times were between 0.5 to $12 \mathrm{~h}$. The mixture was filtered (over Celite, $\mathrm{SiO}_{2}$, or through a fritted funnel) and rinsed with additional $\mathrm{CH}_{2} \mathrm{Cl}_{2}$. The solvent was removed under reduced pressure, and purification by column chromatography afforded corresponding activated esters, which were used without further purification unless otherwise noted. Note: Some esters are prone to hydrolysis on silica gel during column chromatography and should be purified as quickly as possible to obtain reasonable separation.

We have previously reported the synthesis of redox-active esters shown below, including graphical support on their preparation. ${ }^{1,2,3}$ 

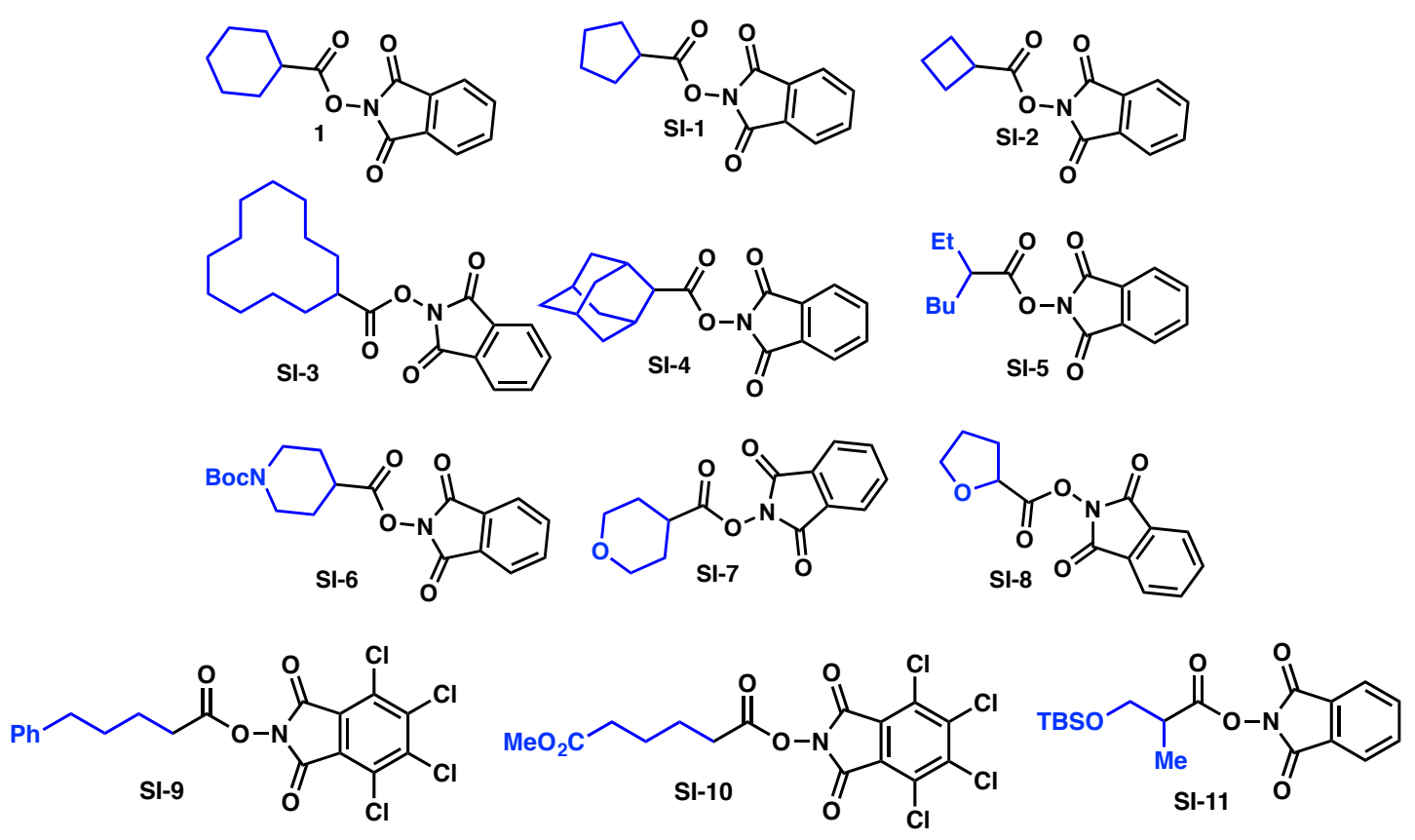<smiles></smiles><smiles>CCCCC/C=C\C/C=C\CCCCCCCC(=O)ON1C(=O)c2c(Cl)c(Cl)c(Cl)c(Cl)c2C1=O</smiles><smiles></smiles><smiles>CC(C)(C)C(=O)ON1C(=O)c2ccccc2C1=O</smiles><smiles>CC1(C(=O)ON2C(=O)c3ccccc3C2=O)CCCCC1</smiles><smiles>O=C(CCCCCBr)ON1C(=O)c2c(Cl)c(Cl)c(Cl)c(Cl)c2C1=O</smiles><smiles>O=C(ON1C(=O)c2c(Cl)c(Cl)c(Cl)c(Cl)c2C1=O)C1CCOCC1C(=O)ON1C(=O)c2c(Cl)c(Cl)c(Cl)c(Cl)c2C1=O</smiles> 


\section{1,3-Dioxoisoindolin-2-yl (R)-2-(1,3-dioxoisoindolin-2-yl)-4-}

methylpentanoate (SI-26).<smiles>CCCCCCC(C)C(=O)ON1C(=O)c2ccccc2C1=O</smiles>

Compound SI-26 was prepared following General Procedure A, using $(R)$-2(1,3-dioxoisoindolin-2-yl)-4-methylpentanoic acid (2.00 g, $7.7 \mathrm{mmol})$. Product SI-26 was isolated by column chromatography (hexanes:ethyl acetate 5:1) to afford $1.7 \mathrm{~g}(54 \%)$ as a white solid.

m.p. $140-141^{\circ} \mathrm{C}$.

$\mathbf{R}_{\boldsymbol{f}}=0.20$ (hexanes:ethyl acetate 5:1).

${ }^{1} \mathrm{H}$ NMR (600 MHz, $\left.\mathrm{CDCl}_{3}\right): \delta 7.92-7.89(\mathrm{~m}, 2 \mathrm{H}), 7.86-7.84(\mathrm{~m}, 2 \mathrm{H}), 7.78-$ $7.74(\mathrm{~m}, 4 \mathrm{H}), 5.37(\mathrm{dd}, J=12.0,4.8 \mathrm{~Hz}, 1 \mathrm{H}), 2.58-2.53(\mathrm{~m}, 1 \mathrm{H}), 2.11-2.06$ $(\mathrm{m}, 1 \mathrm{H}), 1.61-1.54(\mathrm{~m}, 1 \mathrm{H}), 1.01(\mathrm{~d}, J=7.2 \mathrm{~Hz}, 3 \mathrm{H}), 0.97(\mathrm{~d}, J=7.2 \mathrm{~Hz}, 3 \mathrm{H})$. ${ }^{13} \mathrm{C}$ NMR (151 MHz, $\left.\mathrm{CDCl}_{3}\right): \delta$ 167.1, 166.7, 161.5, 134.9, 134.5, 131.8, 128.9, 124.2, 123.9, 49.0, 37.4, 25.0, 23.2, 21.2.

(S)-1-Tert-butyl 2-(1,3-dioxoisoindolin-2-yl) pyrrolidine-1,2-dicarboxylate (SI-27).<smiles>O=C(ON1C(=O)c2ccccc2C1=O)C1CCCN1</smiles>

Compound SI-27 was prepared following General Procedure, using (S)-1(tert-butoxycarbonyl)pyrrolidine-2-carboxylic acid $(2.15 \mathrm{~g}, 10.0 \mathrm{mmol})$. Product SI-27 was isolated by column chromatography (hexanes:ethyl acetate $4: 1$ ) to afford $2.6 \mathrm{~g}(72 \%)$ as a white solid.

$\mathbf{R}_{\boldsymbol{f}}=0.28$ (hexanes:ethyl acetate $4: 1$ )

${ }^{1} \mathrm{H}$ NMR (600 MHz, $\mathrm{CDCl}_{3}$ ): $\delta 7.88(\mathrm{dd}, J=5.4,3.0 \mathrm{~Hz}, 2 \mathrm{H}), 7.79$ (dd, $J=5.4$, $3.0 \mathrm{~Hz}, 2 \mathrm{H}), 4.61(\mathrm{dd}, J=8.4,3.6 \mathrm{~Hz}, 1 \mathrm{H}), 3.65-3.61(\mathrm{~m}, 1 \mathrm{H}), 3.51-3.47$ 
(m, 1H), $2.47-2.40(\mathrm{~m}, 1 \mathrm{H}), 2.38-2.33(\mathrm{~m}, 1 \mathrm{H}), 2.11-2.05(\mathrm{~m}, 1 \mathrm{H}), 2.01-$ $1.95(\mathrm{~m}, 1 \mathrm{H}), 1.51(\mathrm{~s}, 9 \mathrm{H})$.

${ }^{13} \mathrm{C}$ NMR (151 MHz, $\mathrm{CDCl}_{3}$ ): $\delta$ 169.8, 161.8, 153.7, 134.9, 129.1, 124.1, 81.3, $57.3,46.4,31.5,28.2,23.7$.

Spectral data are in accordance with those reported in the literature. ${ }^{4}$

\section{1-(1,3-Dioxoisoindolin-2-yl) 3-methyl cis-cyclopentane-1,3-dicarboxylate} (SI-28).<smiles>CC(=O)C1CCC(C(=O)ON2C(=O)c3ccccc3C2=O)C1</smiles>

Compound SI-28 was prepared following General Procedure A, using cis-3(methoxycarbonyl)cyclopentane-1-carboxylic acid (410 $\mathrm{mg}, 2.38 \mathrm{mmol})$ at 0 ${ }^{\circ} \mathrm{C}$ (reaction run at $0{ }^{\circ} \mathrm{C}$ to hamper the formation of the bis-phthalimide ester, which was observed at r.t.). Product SI-28 was isolated by column chromatography (hexanes:MTBE 0 to $50 \%$ gradient) to afford $641 \mathrm{mg}$ (84\%) as a crystalline solid.

$\mathbf{R}_{\boldsymbol{f}}=0.35$ (silica gel, 2:1 heptane:MTBE)

${ }^{1} \mathrm{H}$ NMR (500 MHz, $\left.\mathrm{CDCl}_{3}\right) \delta 7.92-7.87(\mathrm{~m}, 2 \mathrm{H}), 7.83-7.78(\mathrm{~m}, 2 \mathrm{H}), 3.71$ (s, 3H), $3.24-3.15(\mathrm{~m}, 1 \mathrm{H}), 2.95-2.86(\mathrm{~m}, 1 \mathrm{H}), 2.49-2.41(\mathrm{~m}, 1 \mathrm{H}), 2.37-$ $2.28(\mathrm{~m}, 1 \mathrm{H}), 2.26-2.12(\mathrm{~m}, 2 \mathrm{H}), 2.12-1.99(\mathrm{~m}, 2 \mathrm{H})$.

${ }^{13} \mathrm{C}$ NMR (126 MHz, $\left.\mathrm{CDCl}_{3}\right) \delta 175.0,171.6,162.0,134.8,129.0,124.0,52.0$, 44.0, 40.8, 33.2, 29.4, 29.2. 


\section{1,3-Dioxoisoindolin-2-yl 4-phenyl-2-oxabicyclo[2.2.2]octane-1-}

carboxylate (SI-29).<smiles>O=C1c2ccccc2C(=O)N1OC(=O)C12CCC(c3ccccc3)(CC1)O2</smiles>

Compound SI-29 was prepared following General Procedure A, using 1,3dioxoisoindolin-2-yl 4-phenyl-2-oxabicyclo[2.2.2]octane-1-carboxylate (60 mg, $0.26 \mathrm{mmol})$. Product SI-29 was isolated by column chromatography $\left(\mathrm{CH}_{2} \mathrm{Cl}_{2}\right)$ to afford $71 \mathrm{mg}(78 \%)$ as a white solid.

$\mathbf{R}_{\boldsymbol{f}}=0.45\left(\mathrm{CH}_{2} \mathrm{Cl}_{2}\right)$

m.p. $139-140^{\circ} \mathrm{C}$

${ }^{1} \mathrm{H}-N M R\left(600 \mathrm{MHz} \mathrm{CDCl}_{3}\right) \delta 7.90(\mathrm{dd}, J=5.4,3.1 \mathrm{~Hz}, 2 \mathrm{H}), 7.80(\mathrm{dd}, J=5.5$, $3.1 \mathrm{~Hz}, 2 \mathrm{H}), 7.42(\mathrm{dd}, J=8.4,1.3 \mathrm{~Hz}, 2 \mathrm{H}), 7.34(\mathrm{t}, J=7.8 \mathrm{~Hz}, 2 \mathrm{H}), 7.28-$ $7.22(\mathrm{~m}, 1 \mathrm{H}), 4.36(\mathrm{t}, J=1.4 \mathrm{~Hz}, 2 \mathrm{H}), 2.40-2.27(\mathrm{~m}, 4 \mathrm{H}), 2.24-2.13(\mathrm{~m}$, $4 \mathrm{H})$.

${ }^{13} \mathrm{C}-\mathrm{NMR}\left(151 \mathrm{MHz}, \mathrm{CDCl}_{3}\right) \delta$ 171.2, 162.0, 145.3, 134.9, 129.1, 128.3, 127.2, 124.8, 124.1, 72.6, 69.5, 39.5, 32.6, 28.0.

HRMS (ESI-TOF): calc'd for $\mathrm{C}_{22} \mathrm{H}_{20} \mathrm{NO}_{5}[\mathrm{M}+\mathrm{H}]^{+}$378.1336; found 378.1337.

\section{1-(1,3-Dioxoisoindolin-2-yl) 3-methyl bicyclo[1.1.1]pentane-1,3-} dicarboxylate (SI-30).<smiles>COC(=O)C12CCC(C(=O)ON3C(=O)c4ccccc4C3=O)(CC1[Si])C2</smiles>

Compound SI-30 was prepared following General Procedure A, using 3(methoxycarbonyl)bicyclo[1.1.1]pentane-1-carboxylic acid (150 mg, 0.89 $\mathrm{mmol}$ ). Product SI-30 was isolated by column chromatography (hexanes:ethyl acetate 8:2) to afford $202 \mathrm{mg}(72 \%)$ as a white solid.

$\mathbf{R}_{\boldsymbol{f}}=0.17$ (hexanes:ethyl acetate $4: 1$ )

m.p. $193-197^{\circ} \mathrm{C}$.

${ }^{1} \mathrm{H}-N M R\left(600 \mathrm{MHz}, \mathrm{CDCl}_{3}\right) \delta 7.89(\mathrm{dd}, J=5.5,3.1 \mathrm{~Hz}, 2 \mathrm{H}), 7.80(\mathrm{dd}, J=5.5$, $3.1 \mathrm{~Hz}, 2 \mathrm{H}), 3.72(\mathrm{~s}, 3 \mathrm{H}), 2.56(\mathrm{~s}, 6 \mathrm{H})$. 
${ }^{13}$ C-NMR (151 MHz, $\left.\mathbf{C D C l}_{3}\right) \delta 169.0,164.8,161.8,135.0,129.0,124.2,53.8$, 52.2, 38.7, 35.6.

HRMS (ESI-TOF): calc'd for $\mathrm{C}_{16} \mathrm{H}_{14} \mathrm{NO}_{6}[\mathrm{M}+\mathrm{H}]^{+} 316.0816$; found 316.0816 .

1-(1,3-Dioxoisoindolin-2-yl) 4-methyl cubane-1,4-dicarboxylate (SI-31).<smiles>CC(=O)c1ccccc1C(=O)ON1C(=O)c2ccccc2C1=O</smiles>

Compound SI-31 was prepared following General Procedure A, using 1-(1,3dioxoisoindolin-2-yl) 4-methyl cubane-1,4-dicarboxylate $(1.71 \mathrm{~g}, 8.3 \mathrm{mmol})$. Product SI-31 was isolated by column chromatography $\left(\mathrm{CH}_{2} \mathrm{Cl}_{2}\right.$ :ethyl acetate 10:1), and subsequent crystallization from $\mathrm{CH}_{2} \mathrm{Cl}_{2}$ :hexanes to afford $2.21 \mathrm{~g}$ (76\%) as a white solid.

$\mathbf{R}_{\boldsymbol{f}}=0.66\left(\mathrm{CH}_{2} \mathrm{Cl}_{2}\right.$ :ethyl acetate $\left.10: 1\right)$

m.p. $202-203^{\circ} \mathrm{C}$

${ }^{1} \mathrm{H}-\mathrm{NMR}\left(600 \mathrm{MHz}, \mathrm{CDCl}_{3}\right) \delta 7.89(\mathrm{dd}, J=5.4,3.1 \mathrm{~Hz}, 2 \mathrm{H}), 7.79$ (dd, $J=5.5$, $3.1 \mathrm{~Hz}, 2 \mathrm{H}), 4.51-4.45(\mathrm{~m}, 3 \mathrm{H}), 4.39-4.33(\mathrm{~m}, 3 \mathrm{H}), 3.73(\mathrm{~s}, 3 \mathrm{H})$.

${ }^{13} \mathrm{C}$ NMR (151 MHz, $\left.\mathrm{CDCl}_{3}\right) \delta 171.6,167.0,162.1,134.9,129.1,124.1,55.9$, 53.2, 51.9, 47.8, 47.7.

HRMS (ESI-TOF): calc'd for $\mathrm{C}_{19} \mathrm{H}_{14} \mathrm{NO}_{6}[\mathrm{M}+\mathrm{H}]^{+} 352.0816$; found 352.0817 .

\section{1,3-Dioxoisoindolin-2-yl 1-phenylcyclopropane-1-carboxylate (SI-32).}<smiles>O=C1c2ccccc2C(=O)N1OC(=O)C1(c2ccccc2)CC1</smiles>

Compound SI-32 was prepared following General Procedure A, using 1,3dioxoisoindolin-2-yl 1-phenylcyclopropane-1-carboxylate $(1.62 \mathrm{~g}, 10 \mathrm{mmol}$ ). Product SI-32 was isolated by column chromatography (hexanes: $\mathrm{CH}_{2} \mathrm{Cl}_{2}$ :ethyl acetate 5:1:1.5), and subsequent crystallization from $\mathrm{CH}_{2} \mathrm{Cl}_{2}$ :hexanes to afford $2.54 \mathrm{~g}(83 \%)$ as a white solid.

$\mathbf{R}_{\boldsymbol{f}}=0.45$ (hexanes: $\mathrm{CH}_{2} \mathrm{Cl}_{2}$ :ethyl acetate $5: 1: 1.5$ )

m.p. $176-177^{\circ} \mathrm{C}$ 
${ }^{1} \mathrm{H}-\mathrm{NMR}\left(600 \mathrm{MHz}, \mathrm{CDCl}_{3}\right) \delta 7.85(\mathrm{dd}, J=5.5,3.1 \mathrm{~Hz}, 2 \mathrm{H}$ ), 7.76 (dd, $J=5.5$, $3.1 \mathrm{~Hz}, 2 \mathrm{H}), 7.52(\mathrm{dd}, J=8.2,1.3 \mathrm{~Hz}, 2 \mathrm{H}), 7.37(\mathrm{t}, J=7.4 \mathrm{~Hz}, 2 \mathrm{H}), 7.34-$ $7.29(\mathrm{~m}, 1 \mathrm{H}), 1.91(\mathrm{~m}, 2 \mathrm{H}), 1.52-1.46(\mathrm{~m}, 2 \mathrm{H})$.

${ }^{13} \mathrm{C}-\mathrm{NMR}\left(151 \mathrm{MHz}, \mathrm{CDCl}_{3}\right) \delta 171.2,162.0,137.1,134.8,130.7,129.1$, 128.6, 128.1, 124.0, 123.9, 27.4, 18.8 .

HRMS (ESI-TOF): calc'd for $\mathrm{C}_{18} \mathrm{H}_{14} \mathrm{NO}_{4}[\mathrm{M}+\mathrm{H}]^{+} 308.0917$; found 308.0918 .

1,3-Dioxoisoindolin-2-yl 1-(4-chlorophenyl)cyclopropane-1-carboxylate (SI-33).<smiles>O=C1c2ccccc2C(=O)N1OC(=O)C1(c2ccc(Cl)cc2)CC1</smiles>

Compound SI-33 was prepared following General Procedure A, using 1-(4chlorophenyl)cyclopropanecarboxylic acid $(1.97 \mathrm{~g}, 10.0 \mathrm{mmol})$. Product SI-33 was isolated by column chromatography (hexanes:ethyl acetate $6: 1$ ) to afford $2.7 \mathrm{~g}(75 \%)$ as a white solid.

$\mathbf{R}_{f}=0.32$ (hexanes:ethyl acetate 4:1)

m.p. $139-140^{\circ} \mathrm{C}$

${ }^{1} \mathrm{H}$ NMR (600 MHz, $\left.\mathrm{CDCl}_{3}\right): \delta 7.87-7.84(\mathrm{~m}, 2 \mathrm{H}), 7.78-7.74(\mathrm{~m}, 2 \mathrm{H}), 7.46-$ $7.44(\mathrm{~m}, 2 \mathrm{H}), 7.34-7.32(\mathrm{~m}, 2 \mathrm{H}), 1.91(\mathrm{q}, J=3.6 \mathrm{~Hz}, 2 \mathrm{H}), 1.46$ (q, $J=3.6$ $\mathrm{Hz}, 2 \mathrm{H})$.

${ }^{13} \mathrm{C}$ NMR (151 MHz, $\left.\mathrm{CDCl}_{3}\right): \delta 170.8,161.9,135.6,134.8,134.0,132.1$, 129.0, 128.8, 124.0, 26.8, 18.9.

HRMS (ESI-TOF): calc'd for $\mathrm{C}_{18} \mathrm{H}_{13} \mathrm{CINO}_{4}[\mathrm{M}+\mathrm{H}]^{+} 342.0528$; found 342.0522 . 
1,3-dioxoisoindolin-2-yl 1-(trifluoromethyl)cyclopropane-1-carboxylate (SI-34).<smiles>O=C1c2ccccc2C(=O)N1OC(=O)C1(C(F)(F)F)CC1</smiles>

Compound SI-34 was prepared following General Procedure A, using 1(trifluoromethyl)cyclopropane-1-carboxylic acid $(1.54 \mathrm{~g}, 10 \mathrm{mmol})$. Product SI34 was isolated by column chromatography (hexanes:ethyl acetate 3:1), and subsequent crystallization from $\mathrm{CH}_{2} \mathrm{Cl}_{2}: \mathrm{MeOH}$ to afford $1.80 \mathrm{~g}(60 \%)$ as a white solid.

$\mathbf{R}_{f}=0.43$ (hexanes:ethyl acetate 3:1)

m.p. $105-106^{\circ} \mathrm{C}$

${ }^{1} \mathrm{H}$ NMR (600 MHz, $\left.\mathrm{CDCl}_{3}\right) \delta 7.90-7.86(\mathrm{~m}, 2 \mathrm{H}), 7.81-7.77(\mathrm{~m}, 2 \mathrm{H}), 1.84-$ $1.80(\mathrm{~m}, 2 \mathrm{H}), 1.66-1.62(\mathrm{~m}, 2 \mathrm{H})$.

${ }^{13} \mathrm{C}$ NMR (151 MHz, $\left.\mathrm{CDCl}_{3}\right) \delta 165.2,161.5,135.1,128.8124 .2,123.7$ (q, $J=$ $273.2 \mathrm{~Hz}), 25.7(\mathrm{q}, J=35.9 \mathrm{~Hz}), 14.8$.

${ }^{19} \mathrm{~F}$ NMR (376 $\mathrm{MHz}, \mathrm{CDCl}_{3}$ ) $\delta-67.8$.

HRMS (ESI-TOF): calc'd for $\mathrm{C}_{13} \mathrm{H}_{9} \mathrm{~F}_{3} \mathrm{NO}_{4}[\mathrm{M}+\mathrm{H}]^{+} 300.0478$; found 300.0481 .

1,3-dioxoisoindolin-2-yl 5-phenylpentanoate (SI-35).<smiles>O=C(CCCCc1ccccc1)ON1C(=O)c2ccccc2C1=O</smiles>

Compound SI-35 was prepared following General Procedure A, using 5phenylpentanoic acid $(1.78 \mathrm{~g}, 10 \mathrm{mmol})$. Product SI-35 was isolated by column chromatography (hexanes:ethyl acetate 4:1), and subsequent crystallization from $\mathrm{CH}_{2} \mathrm{Cl}_{2}$ :hexanes to afford $2.35 \mathrm{~g}(73 \%)$ as a colorless needle crystal.

$\mathbf{R}_{f}=0.30$ (hexanes:ethyl acetate $4: 1$ )

m.p. $87-88^{\circ} \mathrm{C}$. 
${ }^{1} \mathrm{H}$ NMR (600 MHz, $\left.\mathrm{CDCl}_{3}\right) \delta 7.88(\mathrm{dd}, J=5.5,3.1 \mathrm{~Hz}, 2 \mathrm{H}), 7.78(\mathrm{dd}, J=5.5$, $3.1 \mathrm{~Hz}, 2 \mathrm{H}), 7.29(\mathrm{t}, J=7.5 \mathrm{~Hz}, 2 \mathrm{H}), 7.23-7.17(\mathrm{~m}, 3 \mathrm{H}), 2.71-2.67(\mathrm{~m}, 4 \mathrm{H})$, $1.87-1.76(\mathrm{~m}, 4 \mathrm{H})$.

${ }^{13} \mathrm{C}$ NMR (151 MHz, $\left.\mathrm{CDCl}_{3}\right) \delta 169.6,163.1,141.8,134.9,129.0,128.5$, 128.5, 126.0, 124.1, 35.5, 31.0, 30.5, 24.4.

\section{General Procedure for the Preparation of Diphenylzinc Reagent 2.}

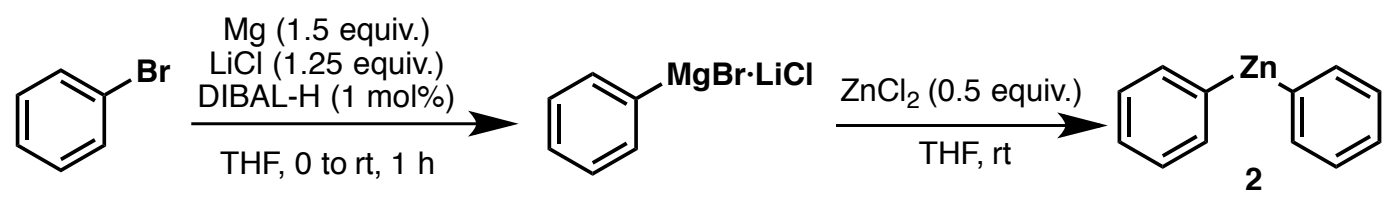

\subsection{Preparation of $\mathrm{PhMgBr} \cdot \mathrm{LiCl}$.}

In an oven-dried $50 \mathrm{~mL}$ 2-necked flask equipped with a double manifold, $\mathrm{Mg}$ turnings (874 $\mathrm{mg}, 36 \mathrm{mmol})$ and $\mathrm{LiCl}(1.27 \mathrm{~g}, 30 \mathrm{mmol})$ were weighed. The flask was sealed, evacuated and back-filled with $\operatorname{Ar}(3$ times). THF (12 mL) was added at r.t. and stirred for 5 minutes. DIBAL-H $(0.24 \mathrm{~mL}, 0.24 \mathrm{mmol}, 1 \mathrm{M}$ in THF) was then added dropwise at r.t. and stirred for 5 minutes. The mixture was then cooled to $0{ }^{\circ} \mathrm{C}$ and $\mathrm{PhBr}(2.52 \mathrm{~mL}, 24 \mathrm{mmol})$ was added dropwise. The mixture was removed from the ice bath and allowed to stir for $1 \mathrm{~h}$ at r.t. Titration of the mixture with $\mathrm{I}_{2}(50 \mathrm{mg}, 0.2 \mathrm{mmol})$ and $\mathrm{LiCl}(1.0 \mathrm{mmol}, 42 \mathrm{mg})$ in THF ( $2 \mathrm{~mL})$ normally affords concentration of about $1-1.3 \mathrm{M}$.

\subsection{Graphical Guide for the Preparation of PhMgBr-LiCl.}
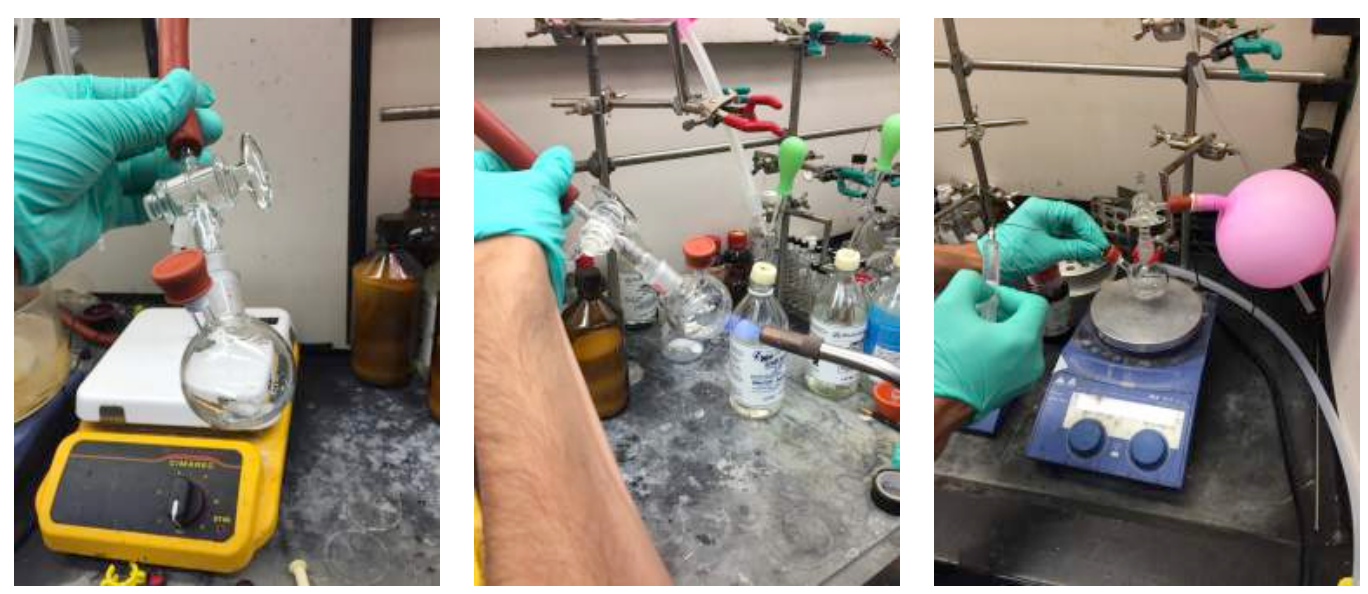

Left: $\mathrm{Mg}$ turnings and $\mathrm{LiCl}$ in a 2-necked flask connected to Ar line. Center: The flask is flame dried with a torch. Right: After cooling down, THF is added. 

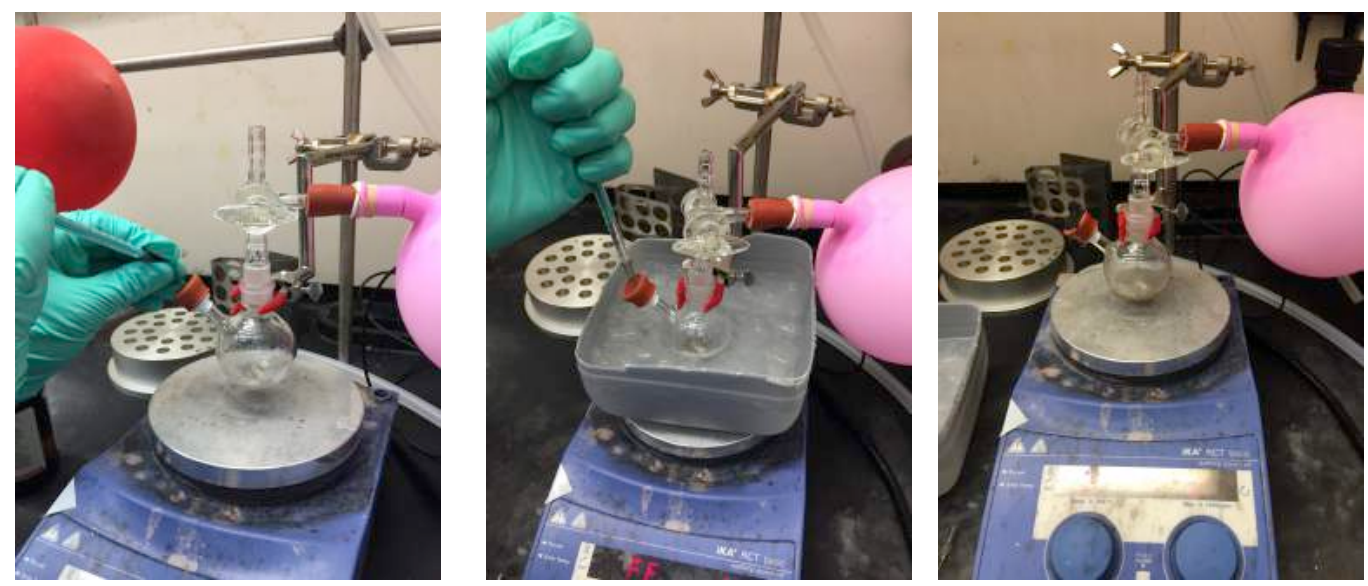

Left: Addition of DiBAL-H (1.0 M) in THF. Center: Addition of $\mathrm{PhBr}$ at $0{ }^{\circ} \mathrm{C}$. Right: After addition of $\mathrm{PhBr}$ and stirred for $1 \mathrm{~h}$ at r.t.
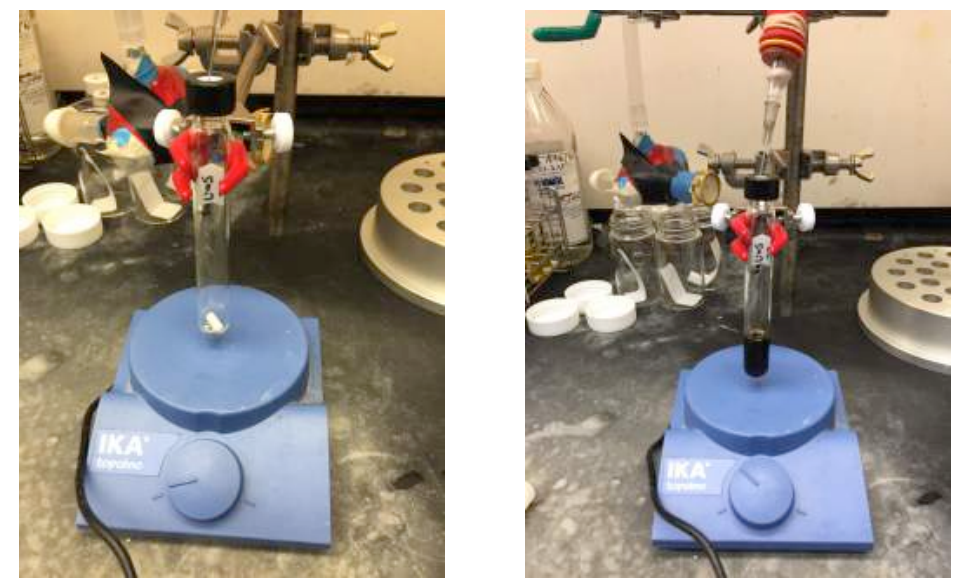

Left: $\mathrm{I}_{2}$ and $\mathrm{LiCl}$ in a culture test tube for titration of the Grignard. Right: Addition of THF.
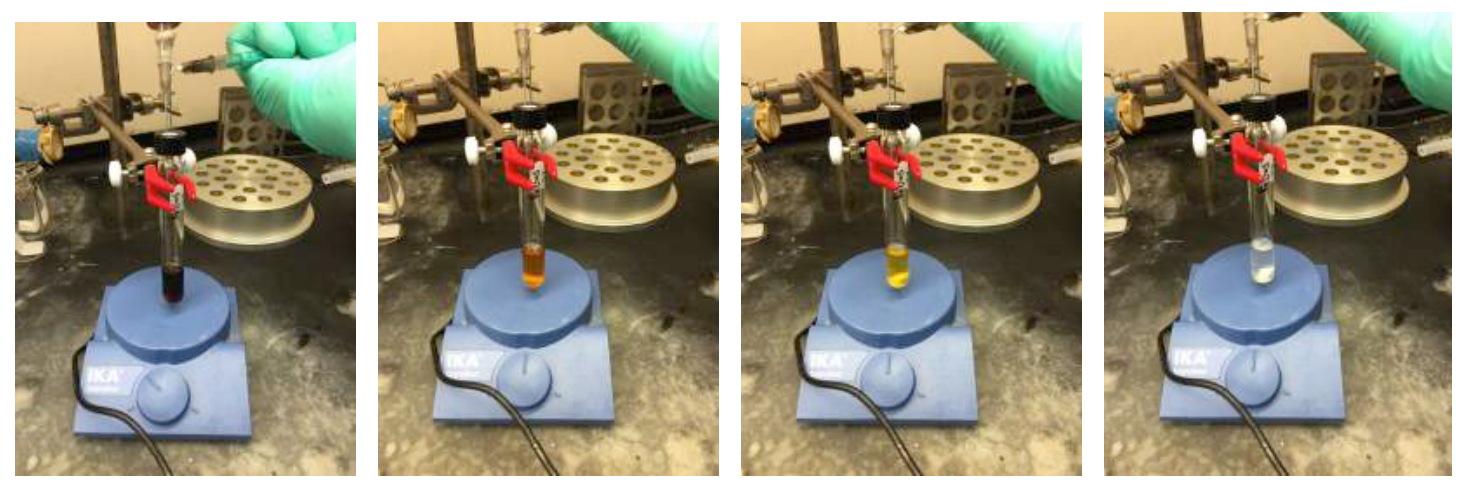

Progress of the titration: Color change upon dropwise addition of $\mathrm{PhMgBr} \cdot \mathrm{LiCl}$ to the $\mathrm{I}_{2}$ solution. 


\subsection{Preparation of $\mathrm{Ph}_{2} \mathrm{Zn}(2)$.}

In an oven-dried 2-necked flask equipped with a double manifold, $\mathrm{ZnCl}_{2}$ was weighed $(680 \mathrm{mg}, 5 \mathrm{mmol})$ and the flask was heated under vaccum for 5 minutes with the help of a heat gun. After cooling down, THF (5 mL) was added under $\mathrm{Ar}$ and the mixture allowed stirring at r.t. for 5 minutes. Then, $\mathrm{PhMgBr} \cdot \mathrm{LiCl}(10 \mathrm{mmol}, 10 \mathrm{~mL}, 1 \mathrm{M})$ was added dropwise. A dense-grey solution was formed and used without further titration $\left(\mathrm{Ph}_{2} \mathrm{Zn}, \mathrm{c}=0.33 \mathrm{M}\right)$.
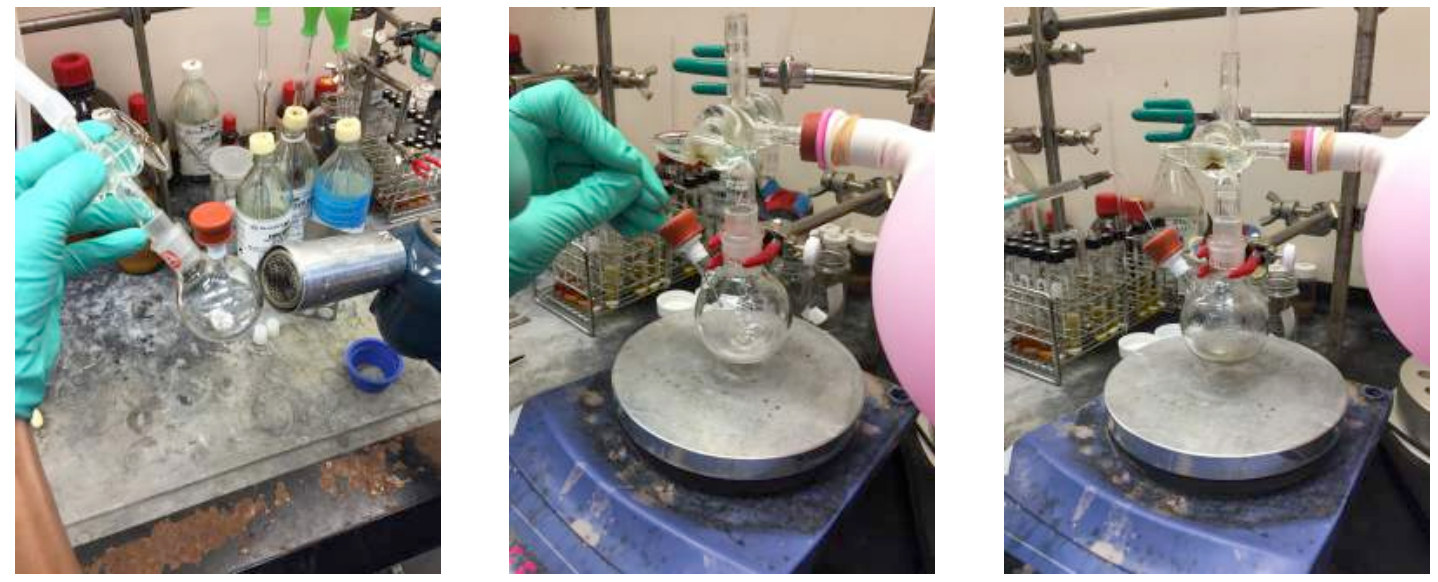

Left: $\mathrm{ZnCl}_{2}$ dried under vacuum with a heatgun. Center: Addition of THF after cooling down.

Right: Addition of $\mathrm{PhMgBr} \cdot \mathrm{LiCl}$. 


\section{Optimization details}

From isolated redox-active ester: A screw-capped culture tube with Teflon ${ }^{\mathrm{TM}}$ septum containing a stirring bar was charged with Fe catalyst, ligand and compound 1 ( $27 \mathrm{mg}, 0.1 \mathrm{mmol}, 1.0$ equiv.) on the bench top. The tube was evacuated and back-filled with Ar. Then, $0.5 \mathrm{~mL}$ of respective solvent were added. The mixture was stirred for 5 minutes under $\mathrm{Ar}$ and $\mathrm{Ph}_{2} \mathrm{Zn}(2)$ in THF was added in one portion at the indicated temperature (do not add it dropwise) to the mixture and stirred at the same temperature. After 1 hour, the reaction was quenched with $1 \mathrm{~N} \mathrm{HCl}$ and diluted with diethyl ether or ethyl acetate. Cyclooctane (internal standard, $0.1 \mathrm{mmol}, 13 \mu \mathrm{L}$ ) was then added to the mixture and an aliquot was analyzed by GC-FID.

From in situ generation of redox-active ester with HATU and HBTU. In a 10 $\mathrm{mL}$ screwed-capped vial cyclohexylcarboxylic acid (13 mg, $0.1 \mathrm{mmol}$ ), HATU (38 mg, $0.1 \mathrm{mmol}$ ) or HBTU (38 mg, $0.1 \mathrm{mmol}$ ) were weighed. The vial was sealed and evacuated and refilled with $\operatorname{Ar} 3$ times. Then, $\mathrm{Et}_{3} \mathrm{~N}(14 \mu \mathrm{L}, 0.1$ $\mathrm{mmol})$ followed by THF $(0.2 \mathrm{~mL})$ were added via syringe under Ar. The mixture was stirred for 2 hours at room temperature (usually, the solution becomes clear after this time). In another $10 \mathrm{~mL}$ screwed-capped vial, $\mathrm{Fe}(\mathrm{acac})_{3}(0.01 \mathrm{mmol})$ and $\mathrm{dppBz}(0.02 \mathrm{mmol})$ were weighed. The vial evacuated and backfilled with Ar 3 times. Then, THF was added $(0.3 \mathrm{~mL})$ to form a red solution. The solution was stirred until all solids were soluble (5 $\min )$. At this point, the mixture was transferred to the redox-active ester vial in one portion at room temperature, followed by the addition of $\mathrm{Ph}_{2} \mathrm{Zn}(0.25$ mmol, 2.5 equiv.) via syringe in one portion (do not add it dropwise). The mixture was stirred for $1 \mathrm{~h}$ at room temperature before quench with $\mathrm{HCl} 1 \mathrm{M}$ and diluted with ether or ethyl acetate. Cyclooctane (internal standard, 0.1 $\mathrm{mmol}, 13 \mu \mathrm{L}$ ) was then added to the mixture and an aliquot was analyzed by GC-FID. 


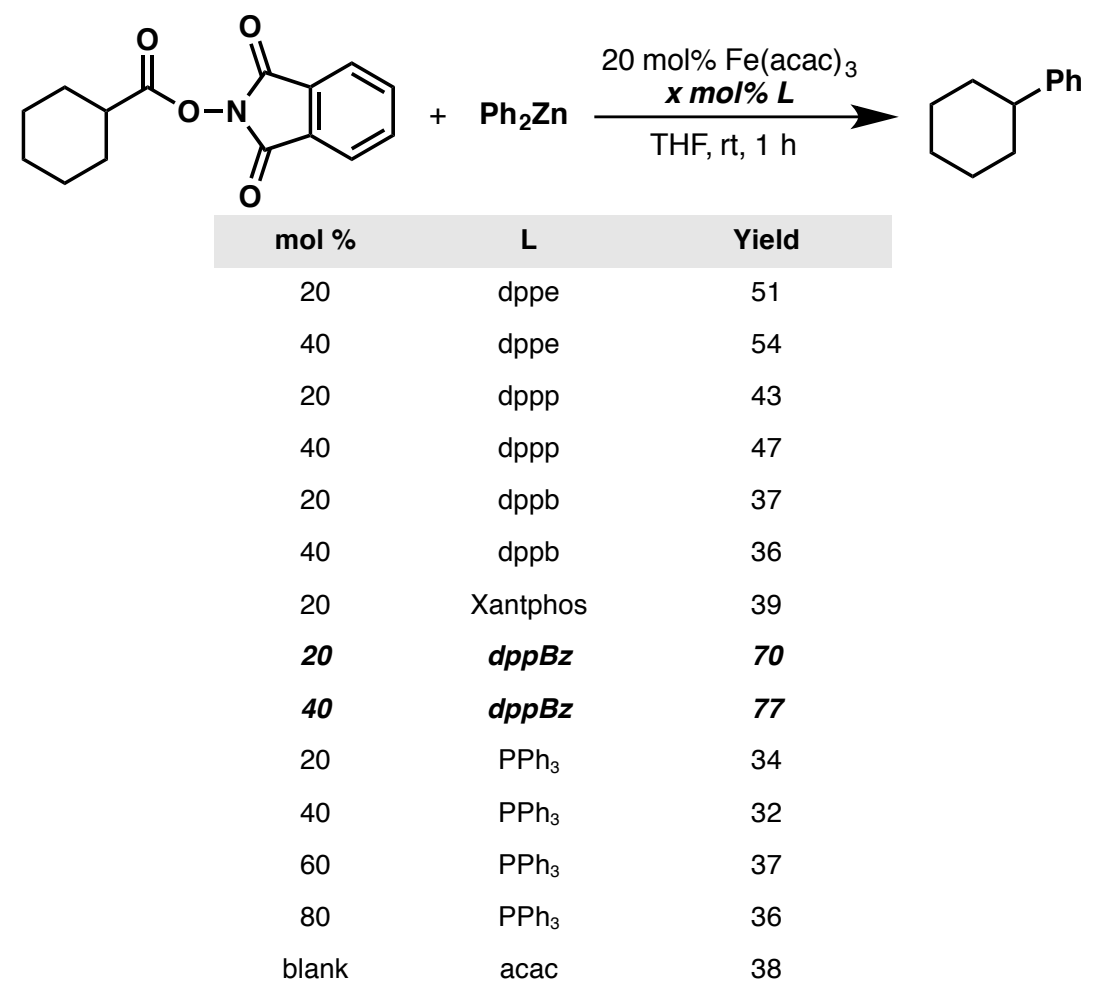

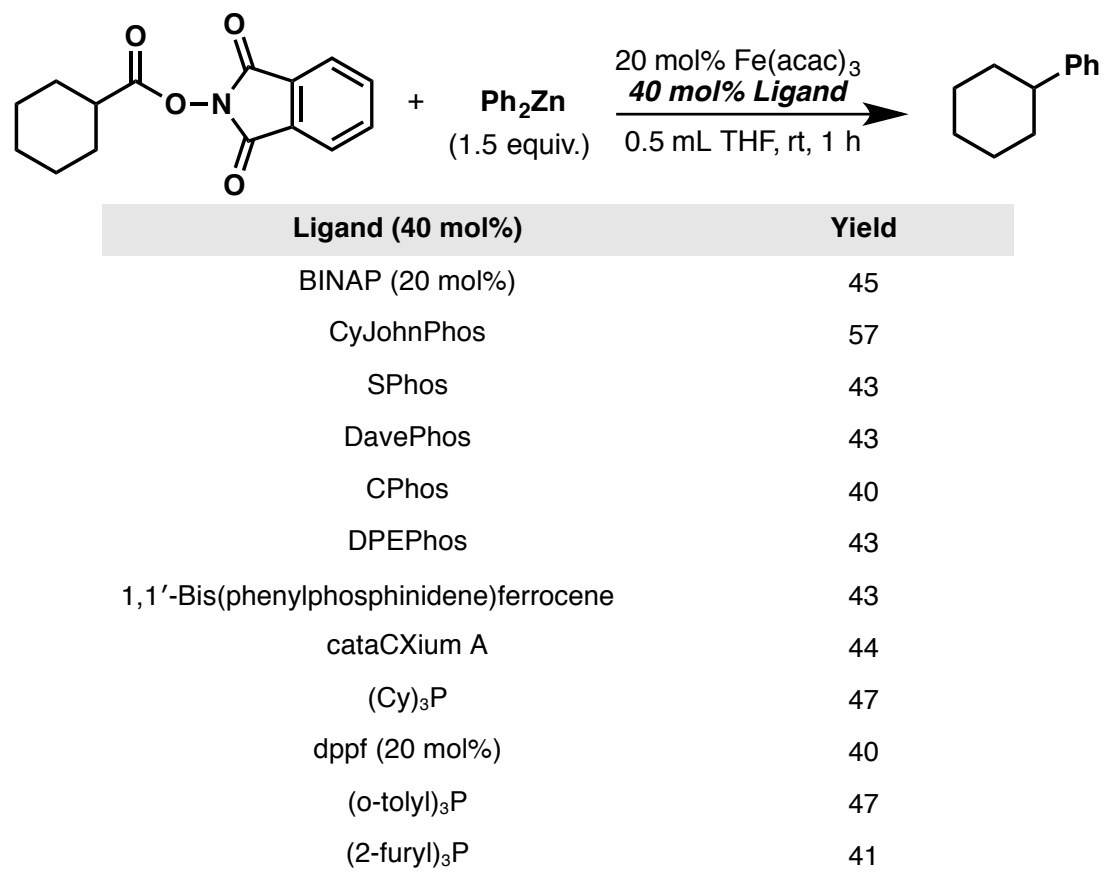




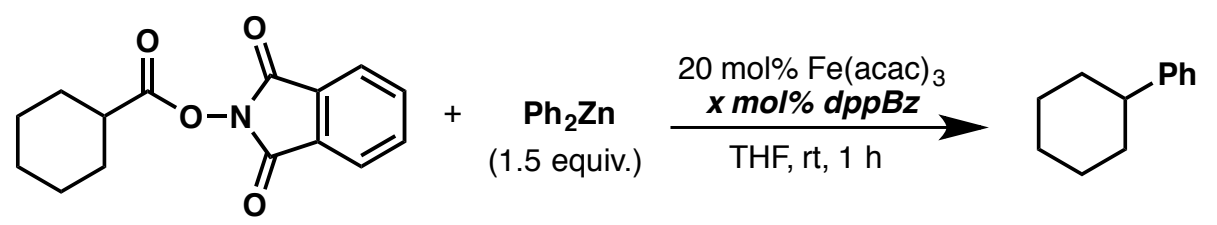

$\begin{array}{cc}\text { dppBz (mol \%) } & \text { Yield } \\ 20 & 74 \\ 22 & 75 \\ 24 & 74 \\ 26 & 75 \\ 28 & 76 \\ 30 & 74 \\ 32 & 73 \\ 34 & 74 \\ 36 & 75 \\ 38 & 75\end{array}$
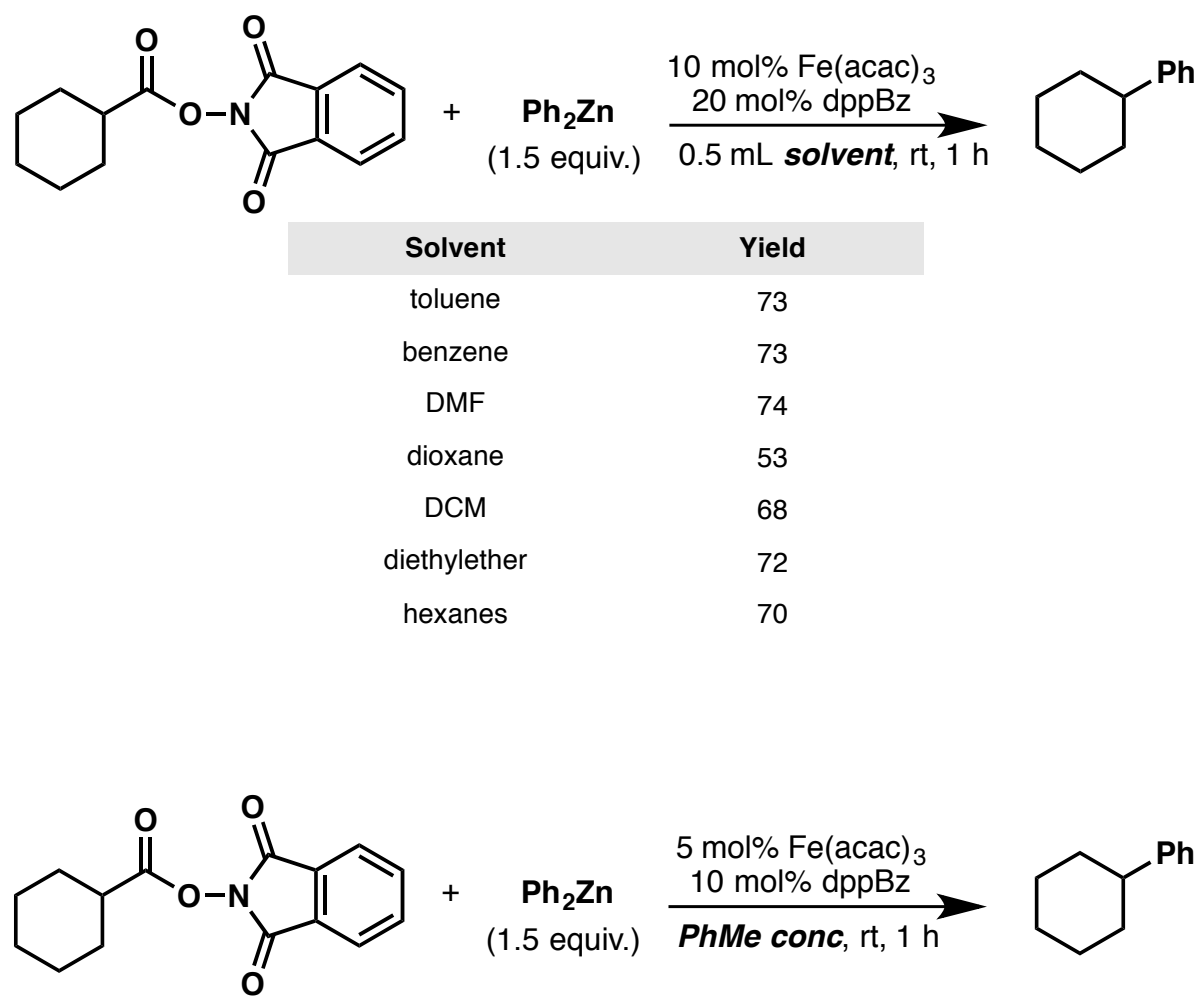

$\begin{array}{cc}\text { conc (PhMe) } & \text { Yield } \\ 0.25 \mathrm{~mL} & 69 \\ 0.5 \mathrm{~mL} & 68 \\ 0.75 \mathrm{~mL} & 68 \\ 1 \mathrm{~mL} & 70 \\ 2 \mathrm{~mL} & 63\end{array}$




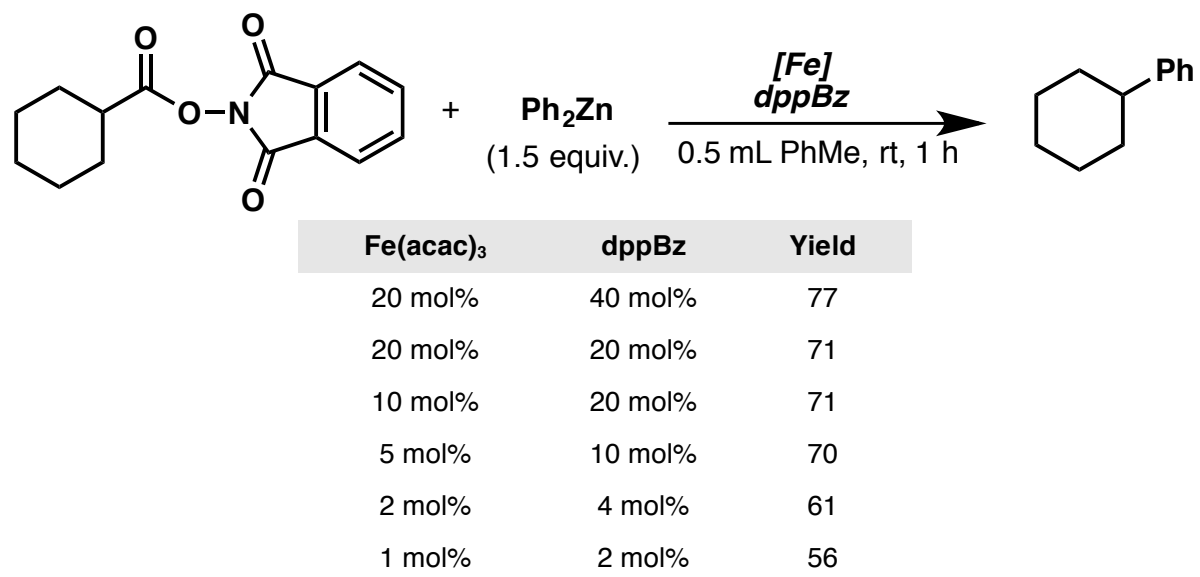




\subsection{Time course of the model reaction.}

Identical reactions were set up following the general procedure for the Fecatalyzed Negishi cross-coupling described for the optimization and quenched at the indicated times of the graphic. As depicted in Graphic S1, the reaction is incredibly fast and in about 5 minutes after the addition of the $\mathrm{Ph}_{2} \mathrm{Zn}$, the reaction is finished. Since we observed different kinetics on different substrates, we standarized the reaction time to 1 hour. It is worth mentioning that the reaction carried out with $\mathrm{Fe}$ affords $75-79 \%$ yield with a $100 \%$ conversion from the redox-active ester. The mass balance corresponds to a $25 \%$ of the starting carboxylic acid, which is hydrolyzed in situ.
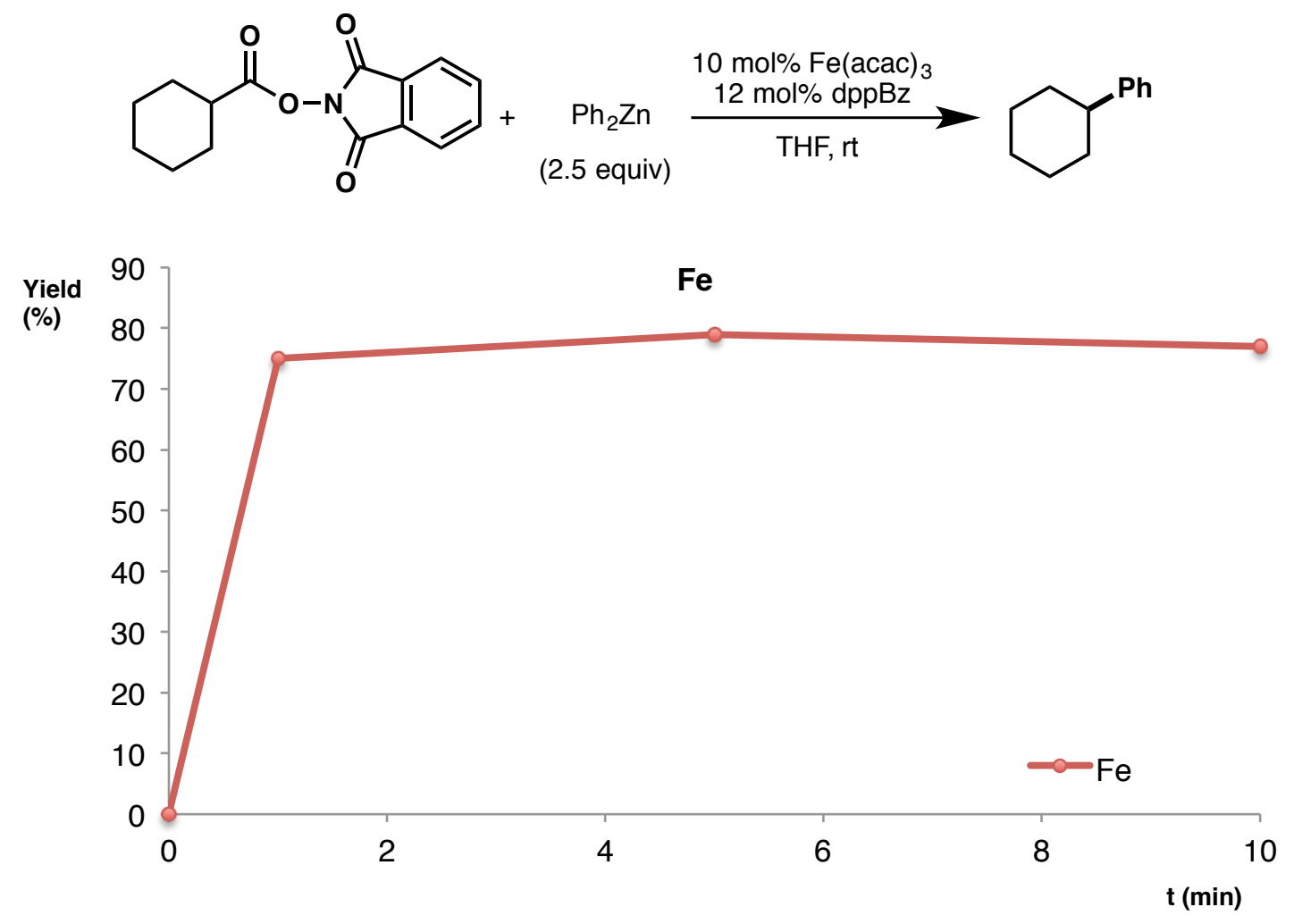

Graphic S1. Kinetic profile for the formation of cyclohexylphenyl (3) with Fe catalysis.

As a comparison, the same reaction was performed but following the protocol for the $\mathrm{Ni}$ catalysis developed by our group. As shown in Graphic S2, the reaction with $\mathrm{Fe}$ is much faster providing higher yields at shorter reaction times when compared to the previously reported reaction using $\mathrm{Ni}$. 

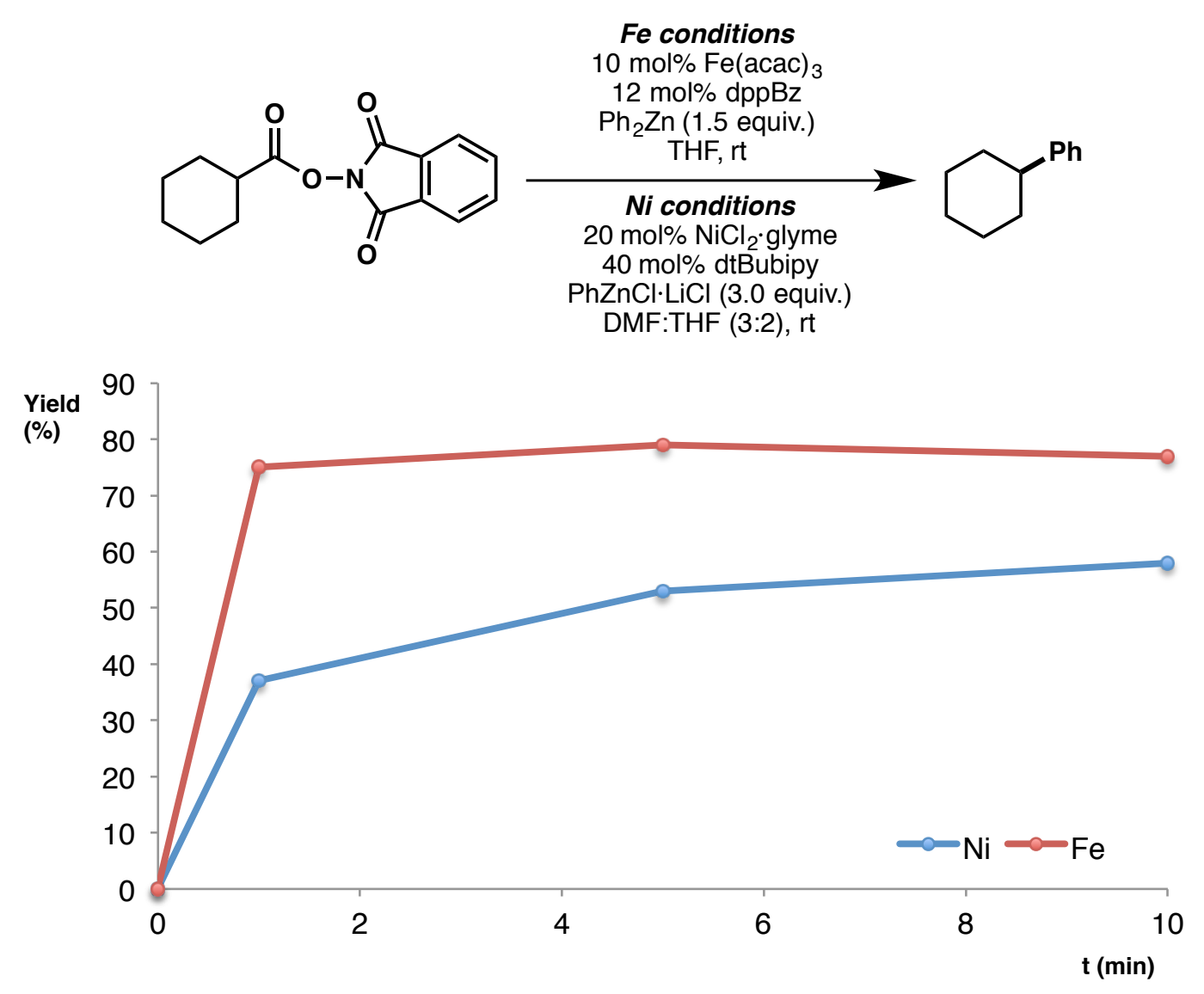

Graphic S2. Comparison of kinetic profiles for the formation of cyclohexylphenyl (3) with Fe and Ni catalysis.

\section{General Procedure for the Fe-catalyzed Negishi cross-coupling with redox-active esters and diarylzinc reagents (General Procedure B).}

In a $10 \mathrm{~mL}$ screwed-capped vial redox-active ester $(0.1 \mathrm{mmol}), \mathrm{Fe}(\mathrm{acac})_{3}$ or $\mathrm{FeCl}_{3}(0.005-0.04 \mathrm{mmol})$ and $\mathrm{dppBz}(0.006-0.048 \mathrm{mmol})$ were weighed. The vial was then sealed, evacuated and back-filled with $\operatorname{Ar}$ (this process was repeated 3 times). Then, $0.5 \mathrm{~mL}$ of distilled THF or toluene were added. The mixture was stirred for 5 minutes under Ar and diarylzinc reagent in THF (0.15 $-0.25 \mathrm{mmol}$, typical concentration after titration is $0.3 \mathrm{M}$ ) was added in one portion at $0-25^{\circ} \mathrm{C}$ (do not add it dropwise) to the mixture and stirred at the same temperature for $1 \mathrm{~h}$. After this time, the reaction was quenched with $\mathrm{HCl}$ $1 \mathrm{~N}$, saturated $\mathrm{NH}_{4} \mathrm{Cl}$ or $\mathrm{H}_{2} \mathrm{O}$ and diluted with diethyl ether. The organic layer was separated and dried over $\mathrm{Na}_{2} \mathrm{SO}_{4}$ anhydrous, filtered and evaporated to 
dryness (for volatile compounds the solvent was carefully evaporated under rotary evaporation at 200 mbar at $25^{\circ} \mathrm{C}$ ). Pure products were obtained after column chromatography or preparative TLC (PTLC).

\subsection{Graphical guide for the Fe-catalyzed Negishi cross-coupling with redox-active esters.}
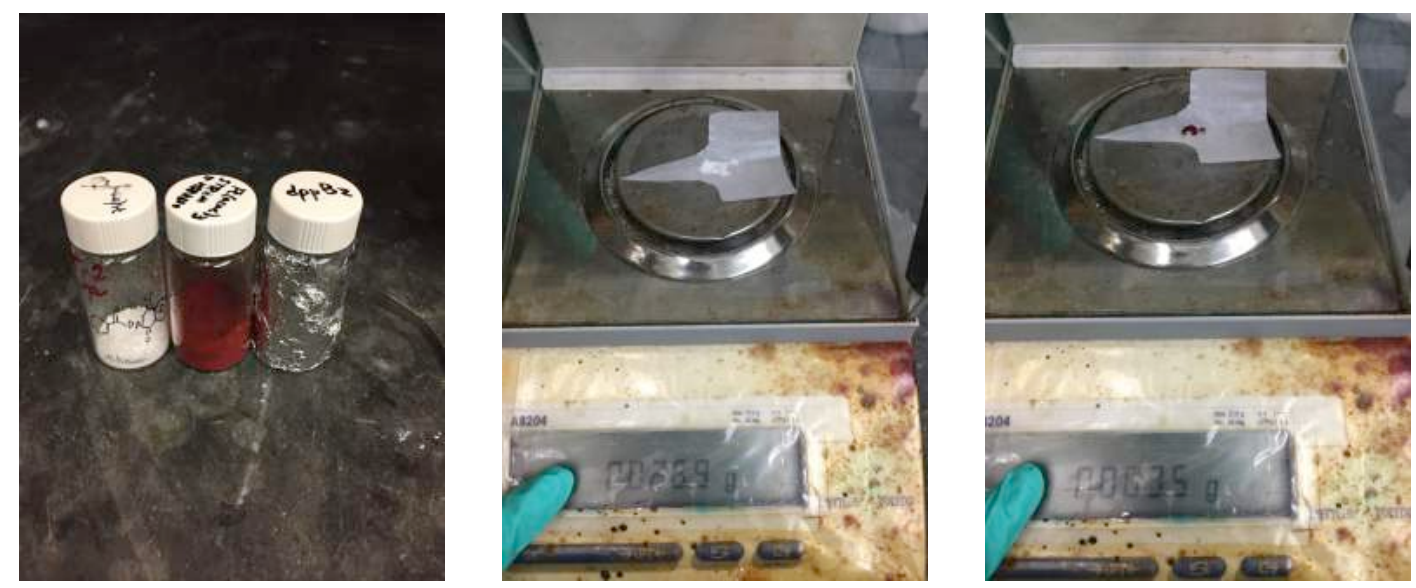

Left: Redox-active ester, $\mathrm{Fe}(\mathrm{acac})_{3}$, dppBz. Center: Redox-active ester weight. Right: $\mathrm{Fe}(\mathrm{acac})_{3}$ weight.
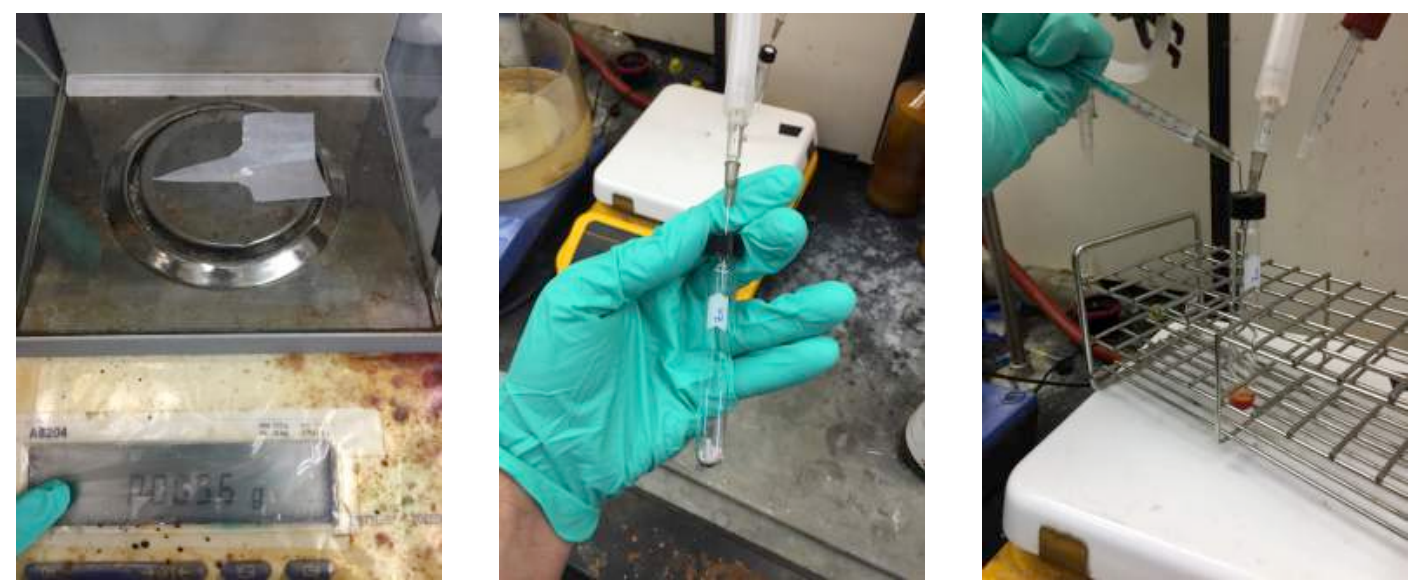

Left: DppBz weight. Center: Reagents in a culture tube under vacuum. Right: Addition of THF to the reaction vessel. 

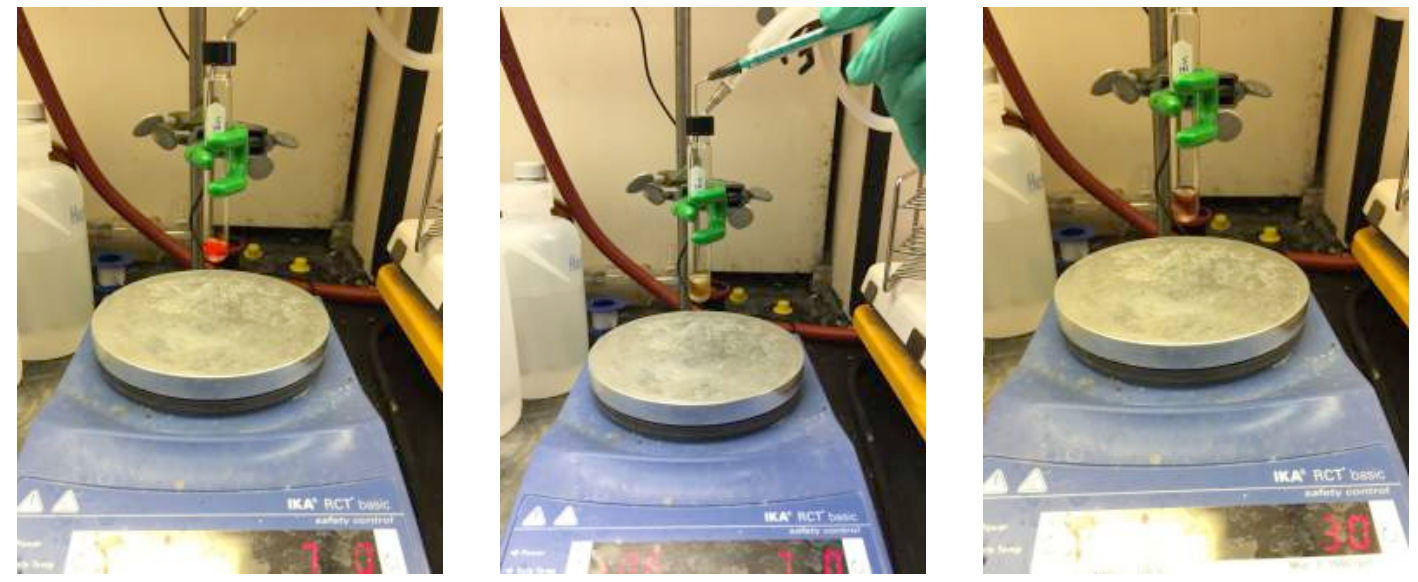

Left: Reaction vessel after addition of THF. Center: Addition of $\mathrm{Ph}_{2} \mathrm{Zn}$. Right: After addition of the $\mathrm{Ph}_{2} \mathrm{Zn}$.
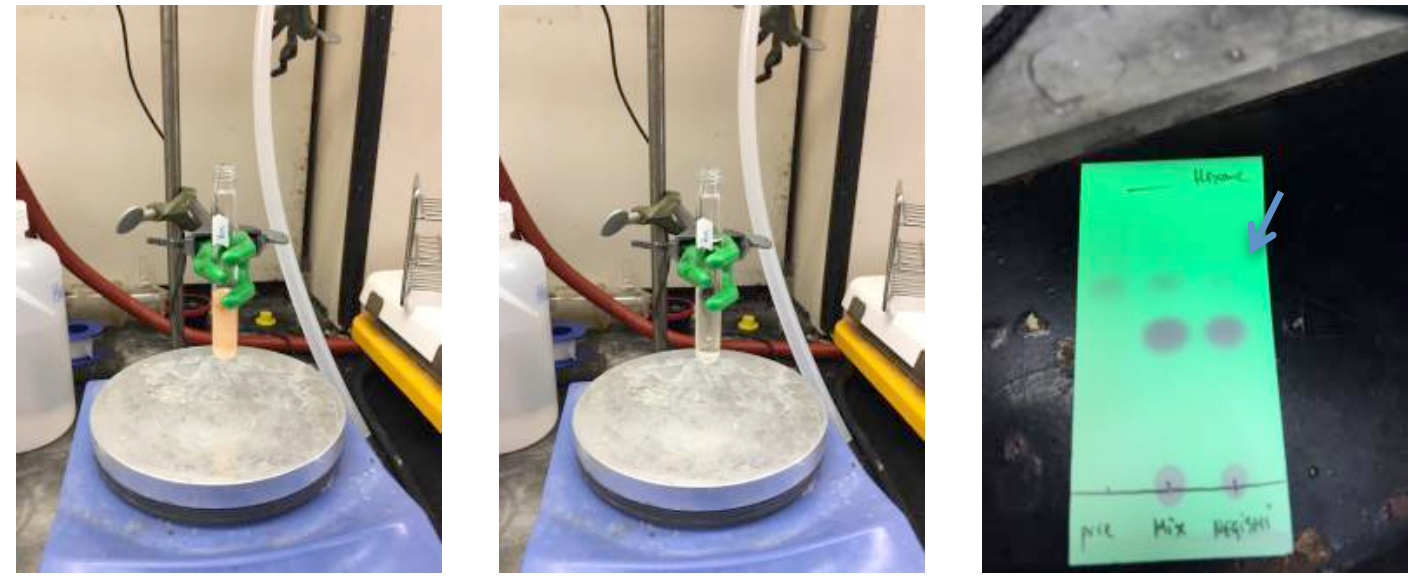

Left. Quench of the reaction after $1 \mathrm{~h}$ with $\mathrm{HCl} 1 \mathrm{M}$. Center: Dilution of the mixture with diethyl ether. Right: TLC plate of the reaction (left: Pure product; center: cross-spot; right: reaction crude).

\section{General Procedure for the Fe-catalyzed Negishi cross-coupling with redox-active esters generated in situ (General Procedure C).}

In a $10 \mathrm{~mL}$ screwed-capped vial carboxylic acid $(0.1 \mathrm{mmol})$, HATU $(38 \mathrm{mg}$, $0.1 \mathrm{mmol}$ ) or HBTU (38 $\mathrm{mg}, 0.1 \mathrm{mmol}$ ) were weighed. The vial was sealed, evacuated and refilled with $\operatorname{Ar} 3$ times. Then, $\mathrm{Et}_{3} \mathrm{~N}(14 \mu \mathrm{L}, 0.1 \mathrm{mmol})$ followed by THF $(0.2 \mathrm{~mL})$ were added via syringe under Ar. The mixture was stirred for $2 \mathrm{~h}$ at room temperature (usually, the solution becomes clear after this time). 
In another $10 \mathrm{~mL}$ screwed-capped vial, $\mathrm{Fe}(\mathrm{acac})_{3}(0.01-0.02 \mathrm{mmol})$ and $\mathrm{dppBz}(0.02-0.04 \mathrm{mmol})$ were weighed. The vial evacuated and backfilled with Ar 3 times. Then, THF $(0.3 \mathrm{~mL})$ was added to form a red solution. The solution was stirred until all solids were soluble $(5 \mathrm{~min})$. At this point, the mixture was transferred to the redox-active ester vial in one portion at $25^{\circ} \mathrm{C}$, followed by the addition of $\mathrm{Ph}_{2} \mathrm{Zn}$ (0.25 mmol, 2.5 equiv.) via syringe in one portion. The mixture was stirred for $1 \mathrm{~h}$ at room temperature before quench with $1 \mathrm{~N} \mathrm{HCl}$, saturated $\mathrm{NH}_{4} \mathrm{Cl}$ or $\mathrm{H}_{2} \mathrm{O}$ and diluted with diethyl ether. The organic layer was separated and dried over $\mathrm{Na}_{2} \mathrm{SO}_{4}$ anhydrous, filtered and evaporated to dryness (for volatile compounds the solvent was carefully evaporated under rotary evaporation at $200 \mathrm{mbar}$ at $25^{\circ} \mathrm{C}$ ). Pure products were obtained after column chromatography or preparative TLC (PTLC).

\subsection{Graphical guide for the Fe-catalyzed Negishi cross-coupling with in situ-formation of the redox-active esters.}
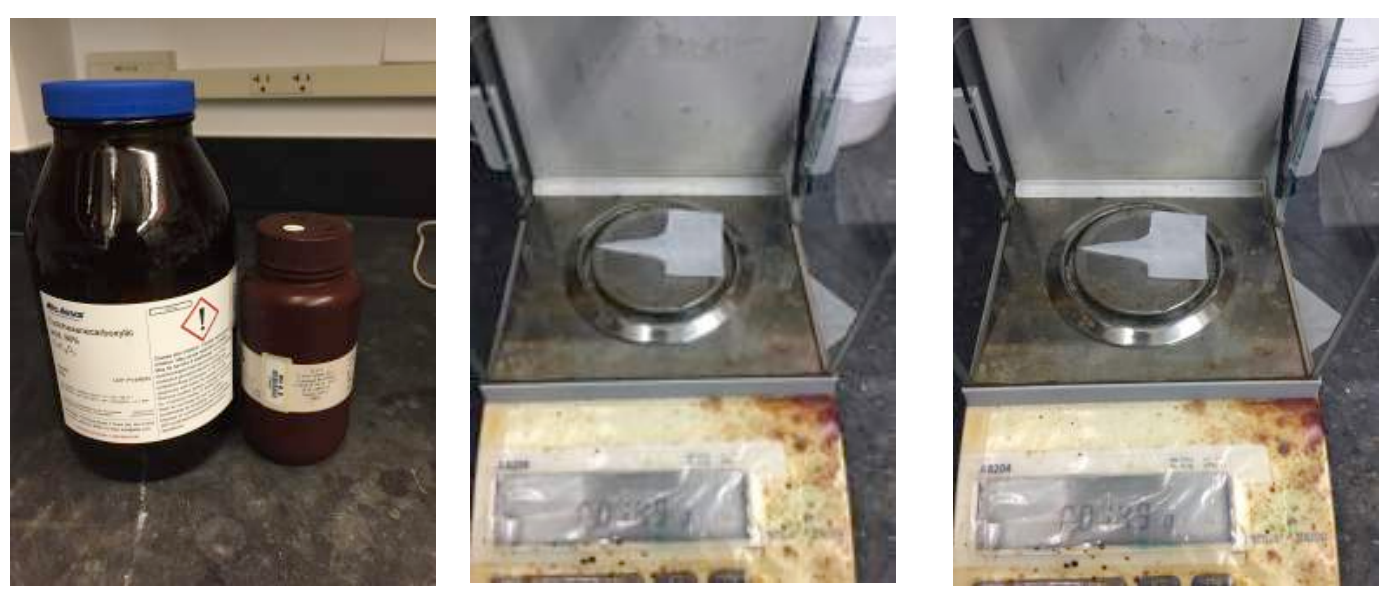

Left: Cyclohexancarboxylic acid and HATU. Center: Weight of Cyclohexancarboxylic acid.

Right: Weight of HATU. 

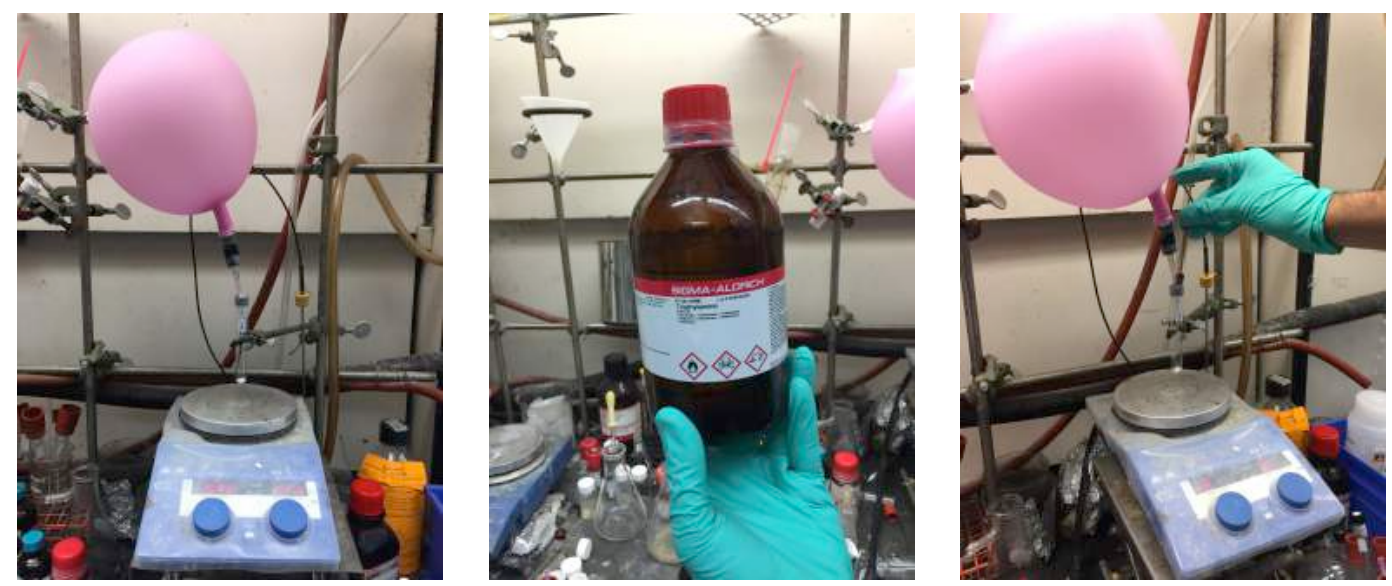

Left: Weighted solids in a culture test tube under Ar. Center: Anhydrous triethylamine utilized. Right: Addition of triethylamine.
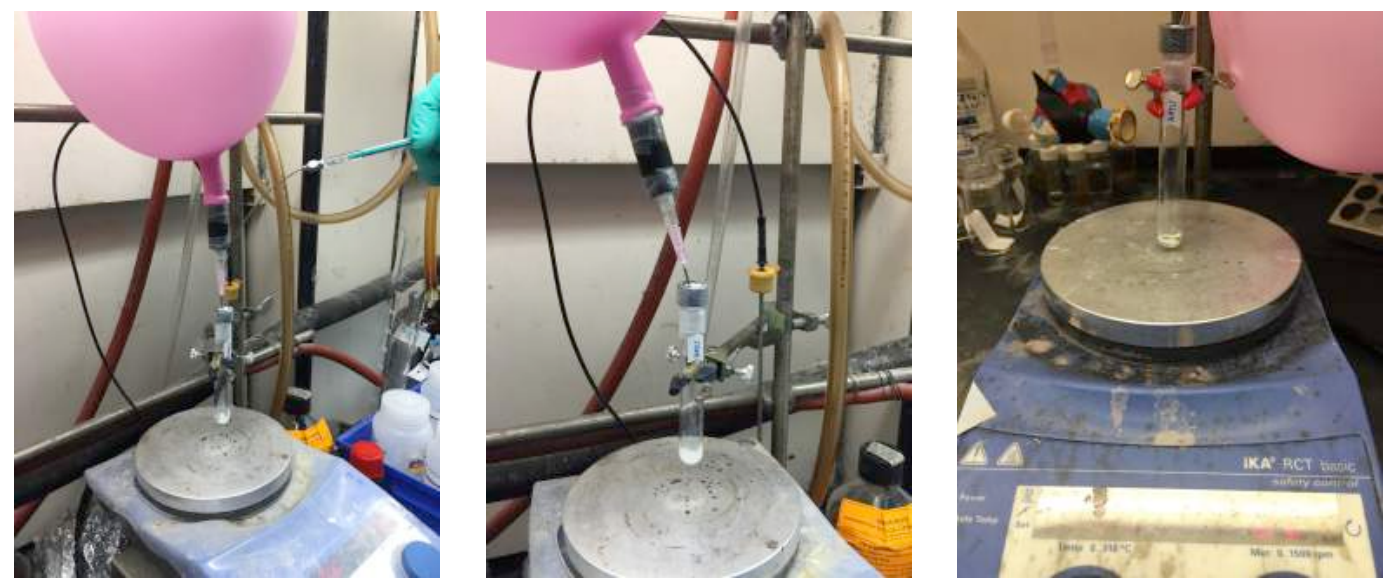

Left: Addition of THF. Center: After addition of THF (white suspension). Right: Solution after 2 hours stirring at r.t. (it becomes a clear solution).
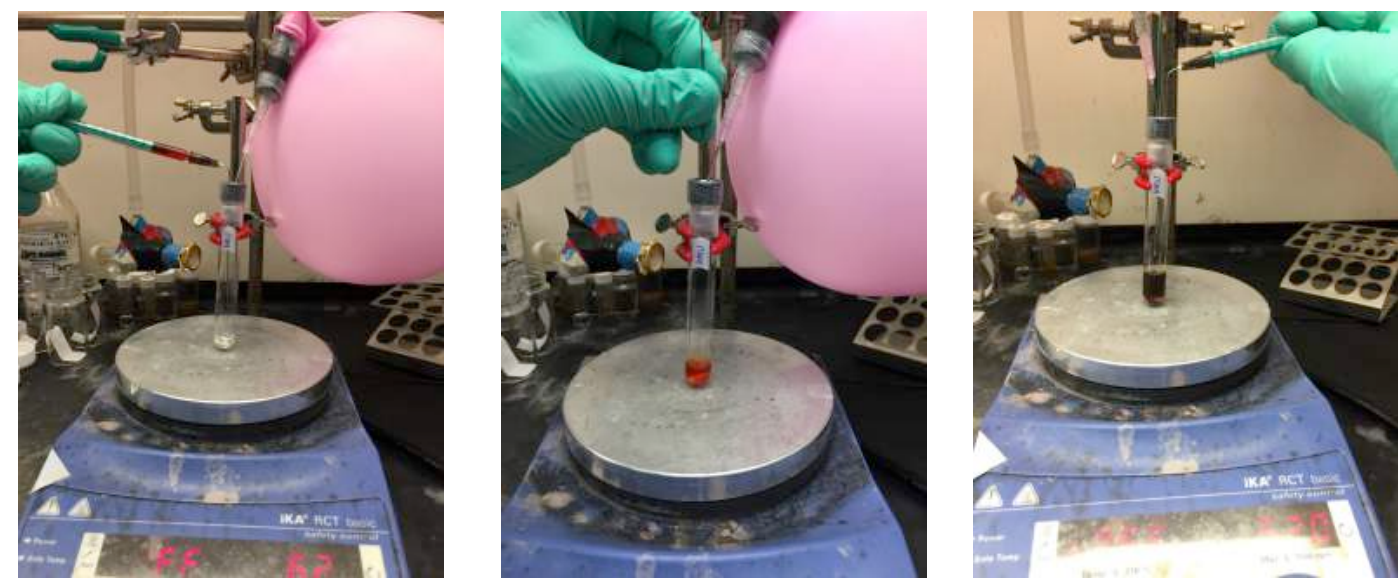

Left: Reaction prior to addition of Fe/ligand solution. Center: Solution after addition of Fe/ligand solution. Right: Addition of $\mathrm{Ph}_{2} \mathrm{Zn}$ in one portion. 

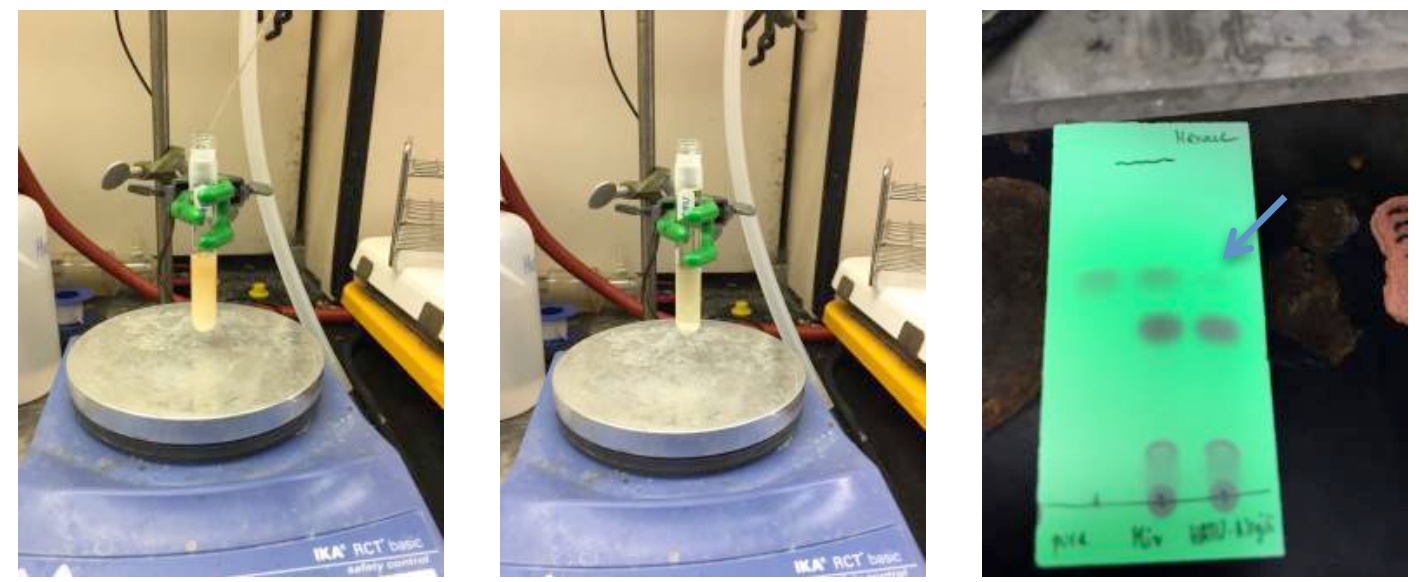

Left. Quench of the reaction after $1 \mathrm{~h}$ with $1 \mathrm{M} \mathrm{HCl}$. Center: Dilution of the mixture with diethyl ether. Right: TLC plate of the reaction (left: Pure product; center: cross-spot; right: reaction crude).

\section{General Procedure for the Fe-catalyzed Kumada-Corriu cross- coupling of redox-active esters and aryl Grignards (General Procedure D).}

In a $10 \mathrm{~mL}$ screwed-capped vial redox-active ester $(0.1 \mathrm{mmol})$ and $\mathrm{Fe}(\mathrm{acac})_{3}$ $(0.02-0.10 \mathrm{mmol})$ were weighed. The vial was then sealed, evacuated and back-filled with $\mathrm{Ar}$ (this process was repeated 3 times). Then, $0.35 \mathrm{~mL}$ of distilled THF and $0.21 \mathrm{~mL}$ of anhydrous DMPU were added. The mixture was stirred for 5 minutes at $25^{\circ} \mathrm{C}$. At this point, $\mathrm{PhMgBr} \cdot \mathrm{LiCl}$ in THF $(0.2-0.3$ $\mathrm{mmol}$, typical concentration after titration is $1.2-1.3 \mathrm{M}$ ) was added in one portion at 0 or $25^{\circ} \mathrm{C}$ (do not add it dropwise) and stirred at this temperature for $1 \mathrm{~h}$. The mixture was quenched with $1 \mathrm{~N} \mathrm{HCl}$, saturated $\mathrm{NH}_{4} \mathrm{Cl}$ or $\mathrm{H}_{2} \mathrm{O}$ and diluted with diethyl ether. The organic layer was separated and dried over $\mathrm{Na}_{2} \mathrm{SO}_{4}$ anhydrous, filtered and evaporated to dryness (for volatile compounds the solvent was carefully evaporated under rotary evaporation at 200 mbar at $25{ }^{\circ} \mathrm{C}$ ). Pure products were obtained after column chromatography or preparative TLC (PTLC). 


\subsection{Graphical guide for the Fe-catalyzed Kumada-Corriu cross-}

\section{coupling.}
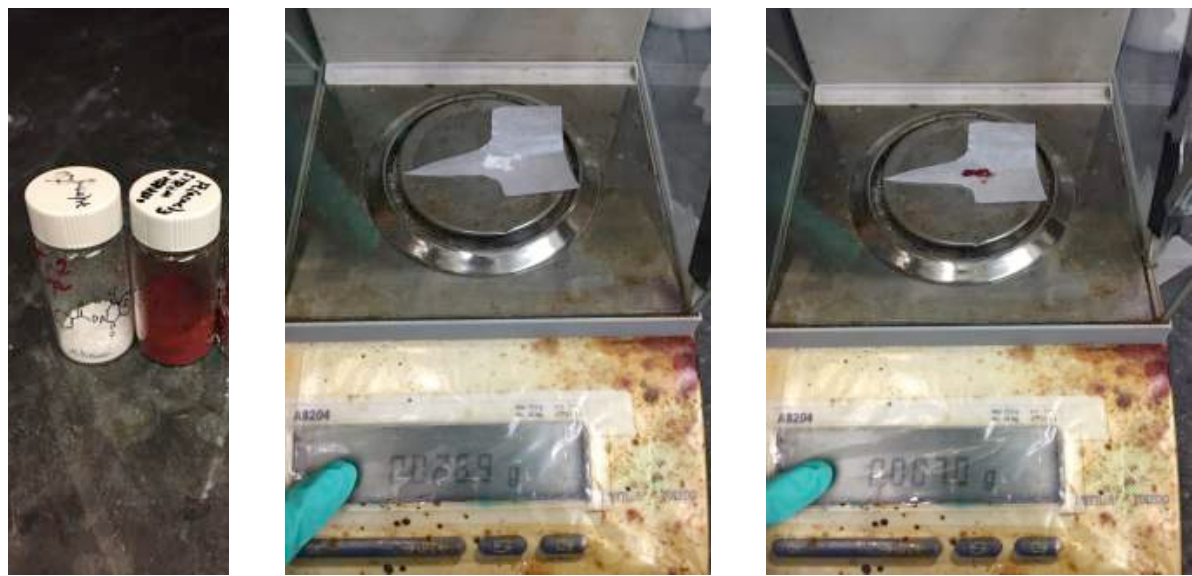

Left: redox-active ester and catalyst. Center: redox-active weight. Right: $\mathrm{Fe}(\mathrm{acac})_{3}$ weight.
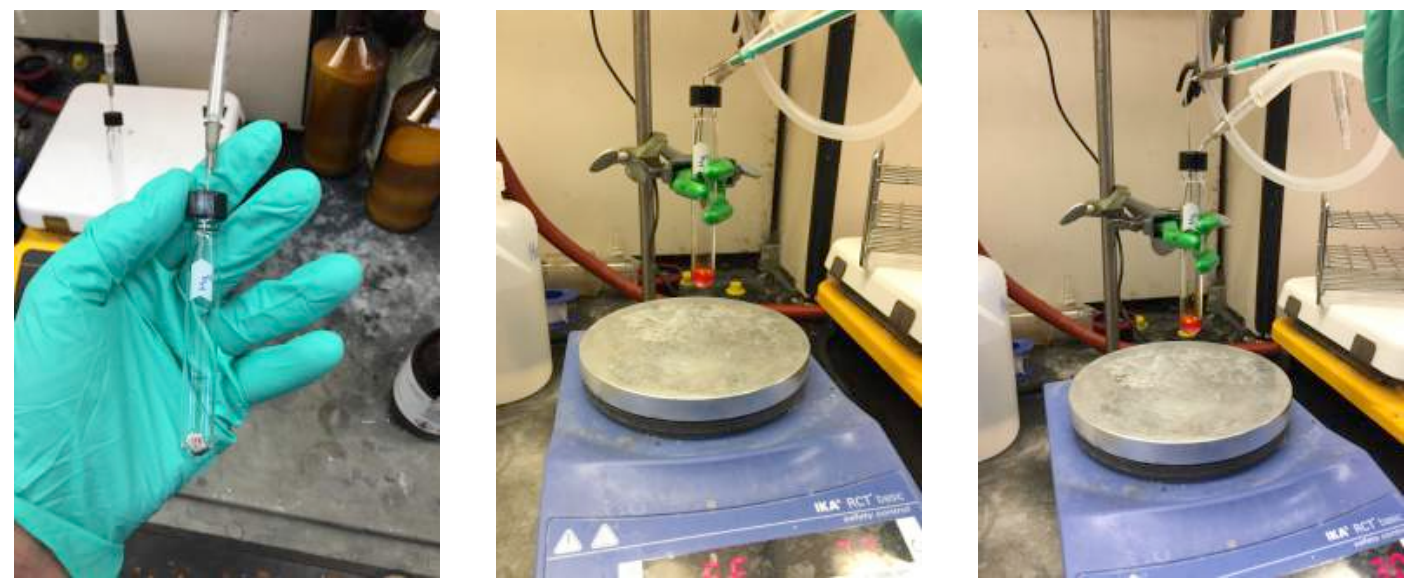

Left: Reagents in a culture tube under vacuum. Center: Addition of THF. Right: Addition of DMPU.
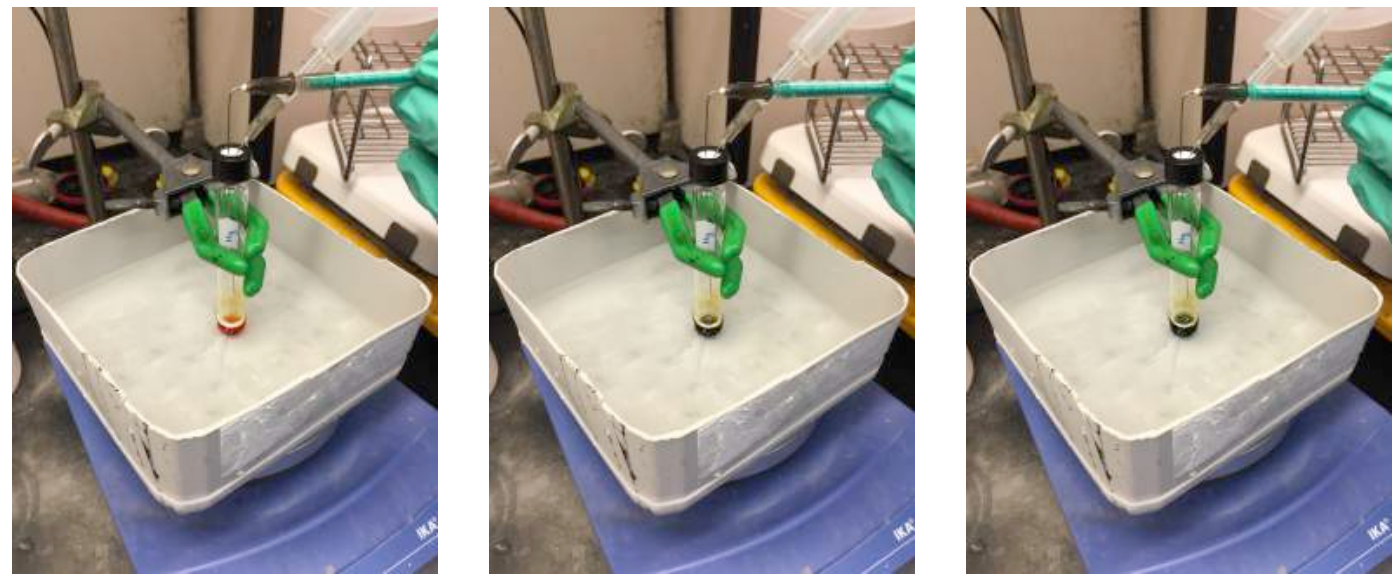

Addition of Grignard. Left: before the addition. Center: during addition. Right: After addition. 

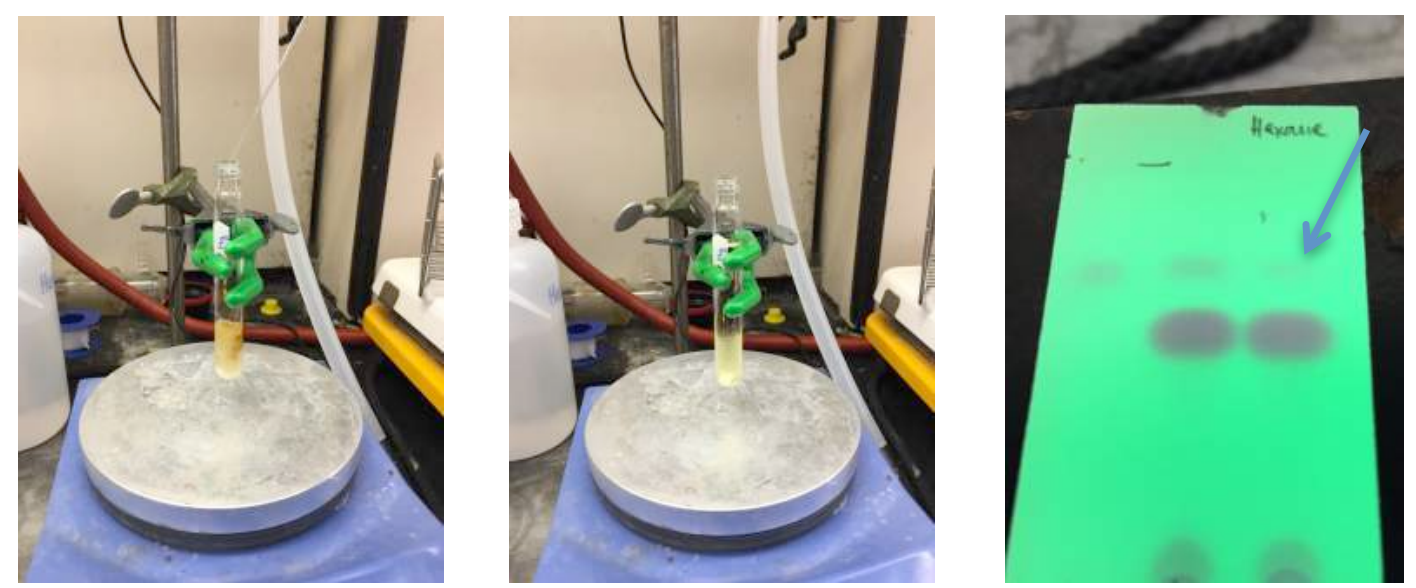

Left: Quench with $\mathrm{HCl} 1 \mathrm{M}$. Center: Dilution with diethyl ether. Right: TLC plate of the reaction (left: Pure product; center: cross-spot; right: reaction crude).

\section{General Procedure for the Ni-catalyzed Negishi cross-coupling of redox-active esters and arylzinc reagents (General Procedure E).}

For detailed information and graphical support on this procedure, see Ref. 1a and $1 \mathrm{~b}$ of the manuscript.

A culture tube with a Teflon ${ }^{\mathrm{TM}}$ septum screw-cap and stir bar was charged with $\mathrm{NiCl}_{2} \cdot$ glyme (20 mol\%), di-tBubipy (40 mol\%) and (if solid) NHPI-ester (1.0 equiv., $0.1 \mathrm{mmol}$ ). The tube was evacuated and backfilled with Ar. Reactions were run with a 3:2 ratio of THF:DMF. The volume of DMF used was calculated based on the titre of the THF solution of the arylzinc chloride reagent. DMF (anhydrous) was added via syringe, and the mixture stirred for 2 minutes at r.t. for solid NHPI-esters. NHPI-ester (if liquid) was dissolved in DMF (anhydrous) and added to the culture tube containing [Ni] and di-tBubipy, and the mixture was stirred for 2 minutes at r.t. Then, arylzinc reagent in THF (3.0 equiv.) was added in one portion, and the mixture was stirred for $12-16$ $h$ at r.t. The mixture was diluted with $\mathrm{EtOAc}$ or $\mathrm{Et}_{2} \mathrm{O}$ and quenched with $1 \mathrm{M}$ $\mathrm{HCl}(\mathrm{aq})$. The reaction can also be quenched with $\mathrm{H}_{2} \mathrm{O}$ or half-saturated $\mathrm{NH}_{4} \mathrm{Cl}$ (aq) solution for acid-sensitive substrates. The organic layer was washed with $\mathrm{H}_{2} \mathrm{O}$ and brine, dried over anhydrous $\mathrm{Na}_{2} \mathrm{SO}_{4}$, and concentrated 
under reduced pressure. The crude material was purified by silica gel column chromatography or preparative TLC.

\section{General Procedure for the Ni-catalyzed Negishi cross-coupling of in situ generated redox-active esters and arylzinc reagents (General Procedure F).}

For detailed information and graphical support on this procedure, see Ref. 1a and $1 \mathrm{~b}$ of the manuscript.

A screw-cap culture tube with Teflon ${ }^{\mathrm{TM}}$ septum and stir bar was charged with carboxylic acid $(0.1 \mathrm{mmol})$ and HATU (38 $\mathrm{mg}, 0.1 \mathrm{mmol}$ ) in DMF (anhydrous, $0.5 \mathrm{~mL}$ ) followed by the addition of TEA (14 $\mu \mathrm{L}, 0.1 \mathrm{mmol})$. The mixture was stir at r.t. for $30 \mathrm{~min}$. A solution of $\mathrm{NiCl}_{2} \cdot g$ lyme (20 mol\%) and di-tBubipy (40 mol\%) in $0.5 \mathrm{~mL}$ of DMF was added to the reaction mixture and stir at r.t. for 5 min. $\mathrm{PhZnCl} \cdot \mathrm{LiCl}$ (in THF, 3 equiv) was added dropwise to the reaction mixture, which was stirred for $12 \mathrm{~h}$. The reaction can also be quenched with $\mathrm{H}_{2} \mathrm{O}$ or half-saturated $\mathrm{NH}_{4} \mathrm{Cl}(\mathrm{aq})$ solution for acid-sensitive substrates. The organic layer was washed with $\mathrm{H}_{2} \mathrm{O}$ and brine, dried over anhydrous $\mathrm{Na}_{2} \mathrm{SO}_{4}$, and concentrated under reduced pressure. The crude material was purified by silica gel column chromatography or preparative TLC. 


\section{Guide for choosing reaction conditions}

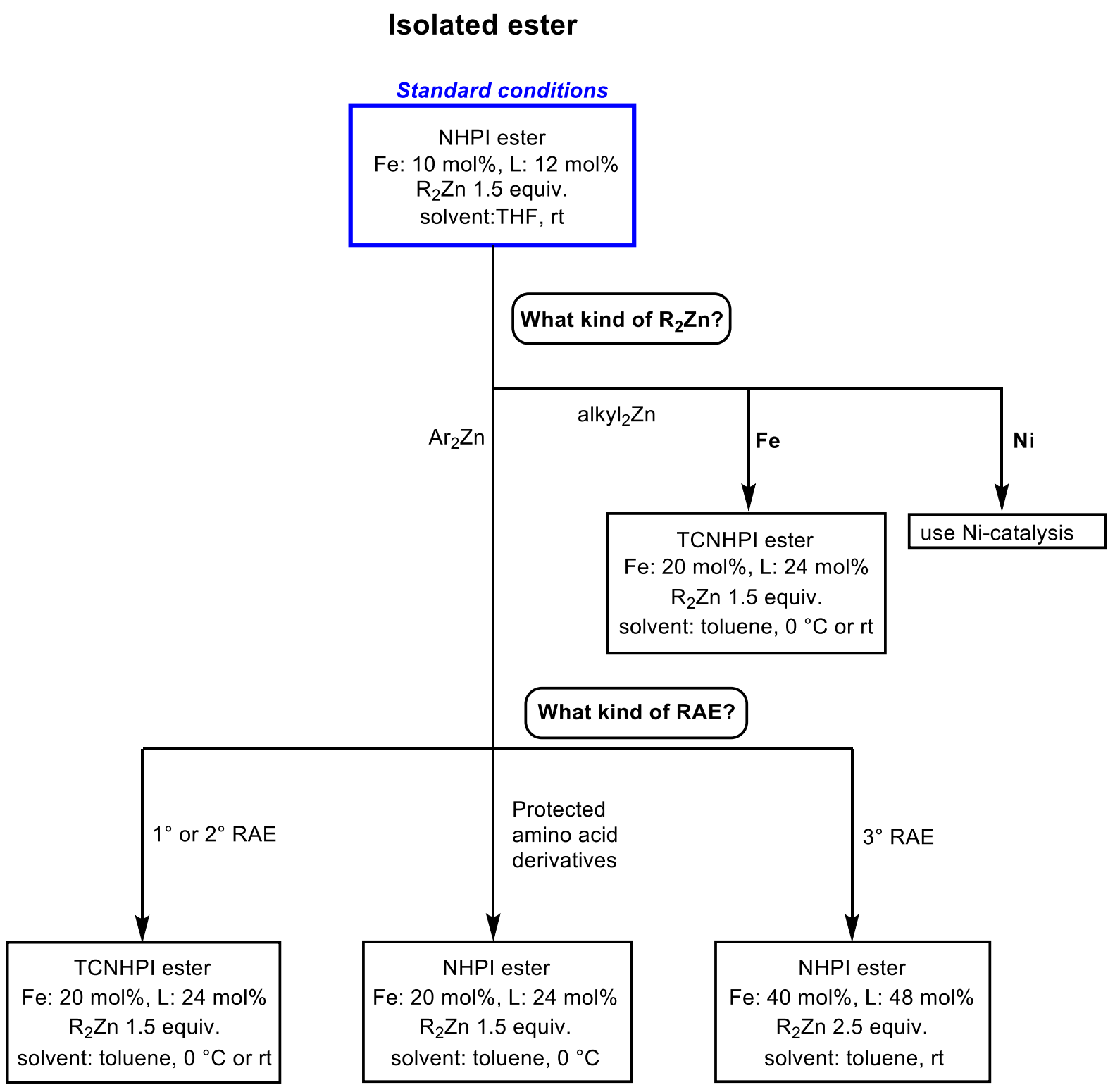




\section{in-situ activation}

\section{from acid}

\section{Standard conditions}

carboxylic acid

HATU 1 equiv, $\mathrm{Et}_{3} \mathrm{~N} 1$ equiv

Fe: $10 \mathrm{~mol} \%$, L: $20 \mathrm{~mol} \%$

$\mathrm{R}_{2} Z \mathrm{n} 2.5$ equiv

solvent: THF, rt

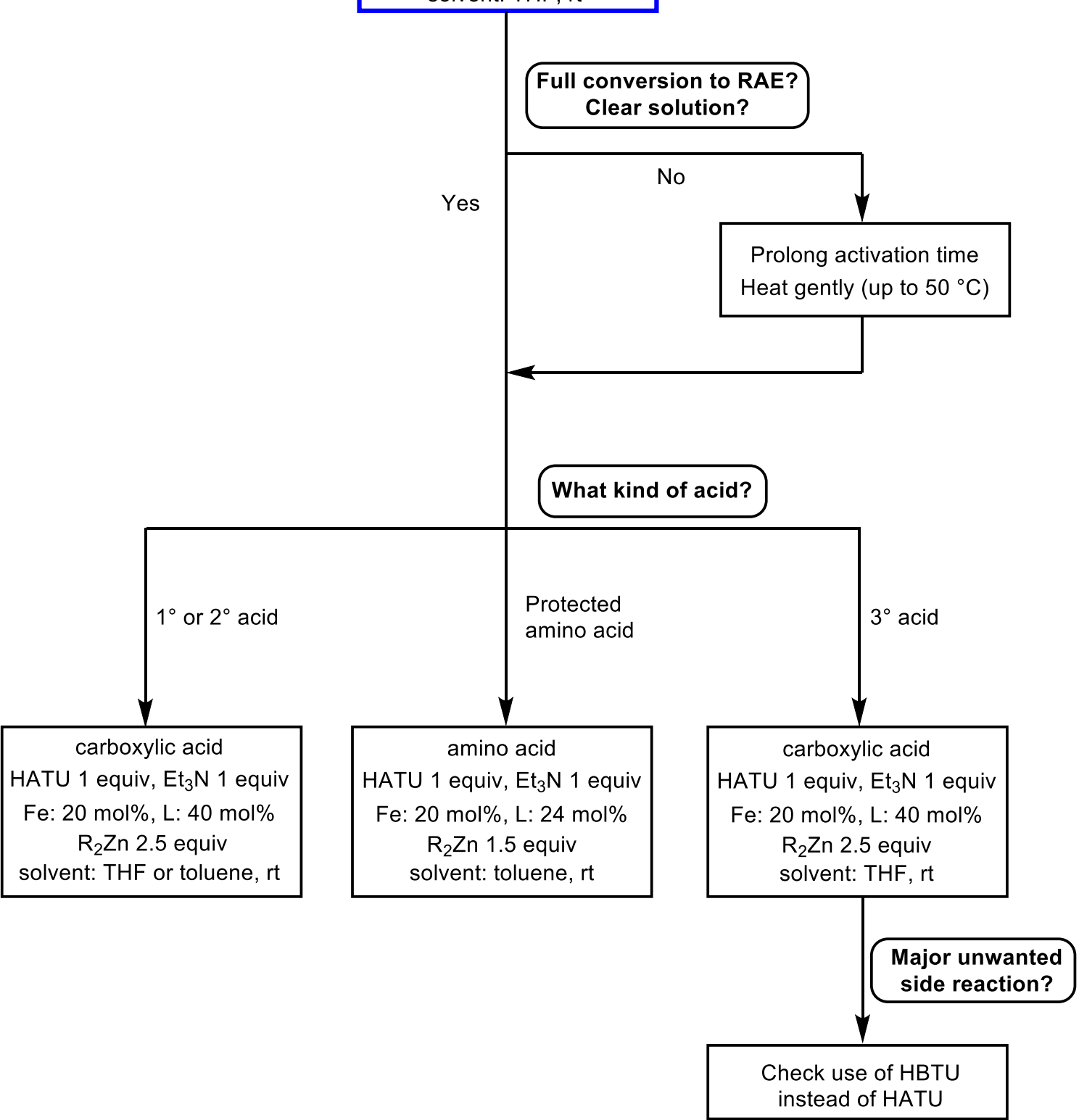




\section{Description of compounds}

Phenylcyclohexane (3).

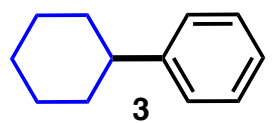

From isolated redox-active ester: Following General Procedure B, with 1 (27 $\mathrm{mg}, 0.1 \mathrm{mmol}), \mathrm{Fe}(\mathrm{acac})_{3}(3.5 \mathrm{mg}, 0.01 \mathrm{mmol}), \mathrm{dppBz}(5.4 \mathrm{mg}, 0.012 \mathrm{mmol})$, $\mathrm{Ph}_{2} \mathrm{Zn}(0.15 \mathrm{mmol})$ at $25^{\circ} \mathrm{C}$. Product 3 was isolated by PTLC (hexanes) to afford $12.2 \mathrm{mg}(75 \%)$ as a transparent oil (note: volatile product).

$1.0 \mathrm{Mmol}$ scale from isolated redox-active ester: Following General Procedure B, with 1 (273 mg, $1.0 \mathrm{mmol}), \mathrm{Fe}(\mathrm{acac})_{3}$ (36 mg, $\left.0.1 \mathrm{mmol}\right), \mathrm{dppBz}$ (54 mg, $0.12 \mathrm{mmol}), \mathrm{Ph}_{2} \mathrm{Zn}(1.5 \mathrm{mmol})$ at $25^{\circ} \mathrm{C}$. Product 3 was isolated by column chromatography (pentane) to afford $130 \mathrm{mg}(81 \%)$ as a transparent oil.

From in situ redox-active ester: Following the General Procedure C with cyclohexancarboxylic acid (13 mg, $0.1 \mathrm{mmol}$ ), HATU (38 mg), $\mathrm{Et}_{3} \mathrm{~N}(14 \mu \mathrm{L})$, $\mathrm{Fe}(\mathrm{acac})_{3}(3.5 \mathrm{mg}, 0.01 \mathrm{mmol}), \mathrm{dppBz}$ (5.4 mg, $\left.0.012 \mathrm{mmol}\right), \mathrm{Ph}_{2} \mathrm{Zn}(0.25$ $\mathrm{mmol}$ ) at $25^{\circ} \mathrm{C}$. Product 3 was isolated by PTLC (hexanes) to afford $11.4 \mathrm{mg}$ $(71 \%)$ as a transparent oil.

Kumada-coupling using Fe-catalysis from isolated redox-active ester. Following General Procedure D: with 1 (27 mg, $0.1 \mathrm{mmol}), \mathrm{Fe}(\mathrm{acac})_{3}(7 \mathrm{mg}$, $0.02 \mathrm{mmol}), \mathrm{PhMgBr} \cdot \mathrm{LiCl}(0.3 \mathrm{mmol})$ at $25^{\circ} \mathrm{C}$. Product 3 was isolated by PTLC (hexanes) to afford $9.9 \mathrm{mg}(61 \%)$ as a transparent oil.

1.0 Mmol scale Kumada-coupling using Fe-catalysis from isolated redoxactive ester. Following General Procedure D: with 1 (273 mg, $1.0 \mathrm{mmol}$ ), $\mathrm{Fe}(\mathrm{acac})_{3}(72 \mathrm{mg}, 0.2 \mathrm{mmol}), \mathrm{PhMgBr} \cdot \mathrm{LiCl}(3.0 \mathrm{mmol})$ at $25^{\circ} \mathrm{C}$. Product 3 was isolated by column chromatography (pentane) to afford $127 \mathrm{mg}(79 \%)$ as a transparent oil. 
Kumada-coupling using Fe-catalysis from in situ generated redox-active ester: Following the General Procedure D with cyclohexancarboxylic acid $(13 \mathrm{mg}$, $0.1 \mathrm{mmol}), \mathrm{HATU}(38 \mathrm{mg}), \mathrm{Et}_{3} \mathrm{~N}(14 \mu \mathrm{L}), \mathrm{Fe}(\mathrm{acac})_{3}(7 \mathrm{mg}, 0.02 \mathrm{mmol})$, $\mathrm{PhMgBr} \cdot \mathrm{LiCl}(0.3 \mathrm{mmol})$ at $25^{\circ} \mathrm{C}$. Product 3 was isolated by PTLC (hexanes) to afford $8.1 \mathrm{mg}(50 \%)$ as a transparent oil.

Kumada-coupling using Ni-catalysis. Following General Procedure D, with 1 (27 mg, $0.1 \mathrm{mmol}), \mathrm{Ni}(\mathrm{acac})_{2}(5.1 \mathrm{mg}, 0.02 \mathrm{mmol}), \mathrm{PhMgBr} \cdot \mathrm{LiCl}(1.5 \mathrm{~mL}, 0.3$ $\mathrm{mmol}$ ) at $25{ }^{\circ} \mathrm{C}$. The yield of product $3(8 \%)$ was determined by NMR using 1,1,2-trichloroethane as internal standard.

Negishi-coupling using Ni-catalysis. Following General Procedure E, with 1 (27 mg, $0.1 \mathrm{mmol}$ ), $\mathrm{NiCl}_{2} \cdot g$ lyme (4.4 mg, $0.02 \mathrm{mmol}$ ), di-tert-butylbipyridine (11 mg, $0.04 \mathrm{mmol}), \mathrm{PhZnCl}(0.3 \mathrm{mmol})$ at $25^{\circ} \mathrm{C}$. The yield of product $3(85 \%)$ was determined by GC using cyclooctane as internal standard.

$\mathbf{R}_{f}=0.78$ (hexanes)

${ }^{1}$ H NMR (600 MHz, $\left.\mathrm{CDCl}_{3}\right) \delta 7.33-7.27(\mathrm{~m}, 2 \mathrm{H}), 7.24-7.20(\mathrm{~m}, 2 \mathrm{H}), 7.19$ (tt, $J=7.2,1.4 \mathrm{~Hz}, 1 \mathrm{H}), 2.51(\mathrm{td}, J=11.5,3.5 \mathrm{~Hz}, 1 \mathrm{H}), 1.91-1.82(\mathrm{~m}, 4 \mathrm{H})$, $1.76(\mathrm{dtt}, J=12.9,3.2,1.6 \mathrm{~Hz}, 1 \mathrm{H}), 1.49-1.33(\mathrm{~m}, 4 \mathrm{H}), 1.31-1.16(\mathrm{~m}, 1 \mathrm{H})$.

${ }^{13} \mathrm{C}$ NMR (151 MHz, $\left.\mathrm{CDCl}_{3}\right) \delta 148.2,128.4,127.0,125.9,44.8,34.6,27.1$, 26.3.

Spectral data are in accordance with those reported in the literature. ${ }^{5}$

\section{1-Cyclohexyl-3-(trifluoromethyl)benzene (4).}

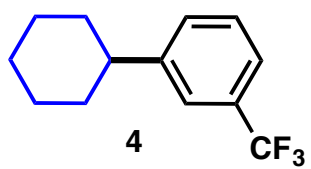

Following the General Procedure B with 1 (27 mg, $0.1 \mathrm{mmol}), \mathrm{Fe}(\mathrm{acac})_{3}(3.5$ $\mathrm{mg}, 0.01 \mathrm{mmol}), \mathrm{dppBz}(5.4 \mathrm{mg}, 0.012 \mathrm{mmol}),\left(3-\left(\mathrm{CF}_{3}\right) \mathrm{Ph}\right)_{2} \mathrm{Zn}(1.25 \mathrm{~mL}, \mathrm{c}=$ $0.12 \mathrm{M}, 0.15 \mathrm{mmol}, 1.5$ equiv) at $25^{\circ} \mathrm{C}$. Product 4 was isolated by PTLC (hexanes/ethyl acetate 9:1) to afford $13.6 \mathrm{mg}(60 \%)$ as transparent oil. 
$\mathbf{R}_{f}=0.65$ (hexanes/ethyl acetate 9:1).

${ }^{1} \mathrm{H}$ NMR (600 MHz, $\left.\mathrm{CDCl}_{3}\right) \delta 7.45(\mathrm{~s}, 1 \mathrm{H}), 7.45-7.41(\mathrm{~m}, 1 \mathrm{H}), 7.41-7.36$ $(\mathrm{m}, 2 \mathrm{H}), 2.63-2.50(\mathrm{~m}, 1 \mathrm{H}), 1.94-1.81(\mathrm{~m}, 4 \mathrm{H}), 1.81-1.71(\mathrm{~m}, 1 \mathrm{H}), 1.49-$ $1.35(\mathrm{~m}, 4 \mathrm{H}), 1.32-1.21(\mathrm{~m}, 1 \mathrm{H})$.

${ }^{13} \mathrm{C}$ NMR (151 MHz, $\left.\mathrm{CDCl}_{3}\right) \delta 149.0,130.7$ (q, $\left.J=31.9 \mathrm{~Hz}\right), 130.4,128.8$, 124.6 (q, $J=272 \mathrm{~Hz}), 123.7$ (q, $J=3.6 \mathrm{~Hz}$ ), 122.8 (q, $J=3.5 \mathrm{~Hz}), 44.6,34.4$, $26.9,26.2$

Spectral data are in accordance with those reported in the literature. ${ }^{6}$ 
4-Cyclopentyl-1,1'-biphenyl (5).

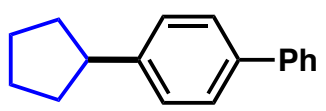

5

Following the General Procedure B with SI-1 (26 mg, $0.1 \mathrm{mmol})$, Fe(acac) 3 (3.5 mg, $0.01 \mathrm{mmol}), \mathrm{dppBz}$ (5.4 mg, $0.012 \mathrm{mmol}),\left(4-(1,1 \text { '-biphenyl) })_{2} \mathrm{Zn}\right.$ (1.27 mL, $0.12 \mathrm{M}, 0.15 \mathrm{mmol}, 1.5$ equiv) at $25^{\circ} \mathrm{C}$. Product 5 was isolated by PTLC (hexanes) to afford $13.7 \mathrm{mg}(62 \%)$ as transparent oil.

$\mathbf{R}_{f}=0.30$ (hexanes).

${ }^{1} \mathrm{H}$ NMR (600 MHz, $\left.\mathbf{C D C l}_{3}\right): \delta 7.60-7.57(\mathrm{~m}, 2 \mathrm{H}), 7.54-7.51(\mathrm{~m}, 2 \mathrm{H}), 7.45-$ $7.40(\mathrm{~m}, 2 \mathrm{H}), 7.35-7.30(\mathrm{~m}, 3 \mathrm{H}), 3.09-2.99(\mathrm{~m}, 1 \mathrm{H}), 2.15-2.05(\mathrm{~m}, 2 \mathrm{H})$, $1.90-1.79(\mathrm{~m}, 2 \mathrm{H}), 1.77-1.67(\mathrm{~m}, 2 \mathrm{H}), 1.67-1.57(\mathrm{~m}, 2 \mathrm{H})$;

${ }^{13} \mathrm{C}$ NMR (150 MHz, $\left.\mathrm{CDCl}_{3}\right): \delta 145.8,141.3,138.8,128.8,127.7,127.2$, 127.1, 127.1, 45.8, 34.8, 25.7.

Spectral data are in accordance with those reported in the literature.1

4-Cyclobutyl-1,1'-biphenyl (6).

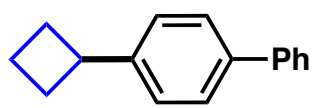

6

Following the General Procedure B with SI-2 (25 mg, $0.1 \mathrm{mmol}), \mathrm{Fe}(\mathrm{acac})_{3}$ (3.5 mg, $0.01 \mathrm{mmol}), \mathrm{dppBz}$ (5.4 mg, $0.012 \mathrm{mmol}),\left(4-(1,1 \text { '-biphenyl) })_{2} \mathrm{Zn}\right.$ (1.27 mL, $0.12 \mathrm{M}, 0.15 \mathrm{mmol}, 1.5$ equiv) at $25^{\circ} \mathrm{C}$. Product 6 was isolated by PTLC (hexanes) to afford $12.9 \mathrm{mg}(62 \%)$ as a transparent oil.

$\mathbf{R}_{f}=0.30$ (hexanes).

${ }^{1} \mathrm{H}$ NMR (600 MHz, $\left.\mathbf{C D C l}_{3}\right): \delta 7.60-7.57(\mathrm{~m}, 2 \mathrm{H}), 7.55-7.52(\mathrm{~m}, 2 \mathrm{H}), 7.45-$ $7.41(\mathrm{~m}, 2 \mathrm{H}), 7.34-7.32(\mathrm{~m}, 1 \mathrm{H}), 7.32-7.28(\mathrm{~m}, 2 \mathrm{H}), 3.64-3.55(\mathrm{~m}, 1 \mathrm{H})$, $2.42-2.32(\mathrm{~m}, 2 \mathrm{H}), 2.25-2.14(\mathrm{~m}, 2 \mathrm{H}), 2.10-1.99(\mathrm{~m}, 1 \mathrm{H}), 1.93-1.84(\mathrm{~m}$, $1 \mathrm{H})$.

${ }^{13} \mathrm{C}$ NMR (150 MHz, $\mathrm{CDCl}_{3}$ ): $\delta$ 145.5, 141.3, 138.8, 128.8, 127.2, 127.1, 127.1, 126.9, 40.2, 30.0, 18.5 . 
Spectral data are in accordance with those reported in the literature.1

\section{Phenylcyclododecane (7).}

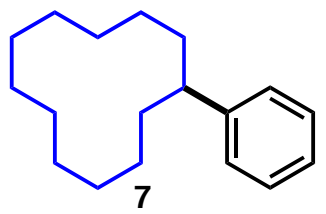

From isolated redox-active ester: Following General Procedure B, with SI-3 (36 mg, $0.1 \mathrm{mmol}), \mathrm{Fe}(\mathrm{acac})_{3}(3.5 \mathrm{mg}, 0.01 \mathrm{mmol}), \mathrm{dppBz}(5.4 \mathrm{mg}, 0.012$ $\mathrm{mmol}), \mathrm{Ph}_{2} \mathrm{Zn}(0.15 \mathrm{mmol})$ at $25{ }^{\circ} \mathrm{C}$. Product 7 was isolated by PTLC (hexanes) to afford $18 \mathrm{mg} \mathrm{(74 \% )}$ as a white solid.

$\boldsymbol{R}_{\boldsymbol{f}}=0.76$ (hexanes).

'H NMR (600 MHz, $\left.\mathrm{CDCl}_{3}\right): \delta 7.32$ (t, $\left.J=7.6 \mathrm{~Hz}, 2 \mathrm{H}\right), 7.26-7.17(\mathrm{~m}, 3 \mathrm{H})$, $2.80(\mathrm{p}, J=6.5 \mathrm{~Hz}, 1 \mathrm{H}), 1.94-1.71(\mathrm{~m}, 2 \mathrm{H}), 1.64-1.00(\mathrm{~m}, 2 \mathrm{H})$.

$\left.{ }^{13} \mathrm{C} \mathrm{NMR} \mathrm{(151} \mathrm{MHz,} \mathrm{CDCl}_{3}\right): \delta 147.2,127.7,127.2,125.2,39.2,31.1,23.5$, 23.5, 23.0, 22.8, 22.3.

Spectral data are in accordance with those reported in the literature.1

\section{2-Phenyladamantane (8).}<smiles>c1ccc(C2C3CC4CC(C3)C2C4)cc1</smiles>

From isolated redox-active ester: Following General Procedure B, with SI-4 (33 mg, $0.1 \mathrm{mmol}), \mathrm{Fe}(\mathrm{acac})_{3}(3.5 \mathrm{mg}, 0.01 \mathrm{mmol}), \mathrm{dppBz}(5.4 \mathrm{mg}, 0.012$ $\mathrm{mmol}), \mathrm{Ph}_{2} \mathrm{Zn}(0.15 \mathrm{mmol})$ at $25^{\circ} \mathrm{C}$. Product 8 was isolated by PTLC (hexanes) to afford $14 \mathrm{mg}(67 \%)$ as a white solid.

$\mathbf{R}_{f}=0.69$ (Hexanes);

${ }^{1} \mathrm{H}$ NMR (400 MHz, $\left.\mathrm{CDCl}_{3}\right): \delta 7.45-7.31(\mathrm{~m}, 4 \mathrm{H}), 7.22(\mathrm{t}, J=7.1 \mathrm{~Hz}, 1 \mathrm{H})$, $3.06(\mathrm{~s}, 1 \mathrm{H}), 2.52(\mathrm{~s}, 2 \mathrm{H}), 2.09-1.74(\mathrm{~m}, 10 \mathrm{H}), 1.60(\mathrm{~d}, J=12.5 \mathrm{~Hz}, 2 \mathrm{H})$.

${ }^{13} \mathrm{C}$ NMR (101 MHz, $\left.\mathrm{CDCl}_{3}\right): \delta 144.4,128.1,126.9,125.2,46.8,39.2,37.9$, $32.0,31.1,28.1,27.8$.

Spectral data are in accordance with those reported in the literature.1 
Heptan-3-ylbenzene (9).<smiles>CCC(C(=O)c1ccccc1)c1ccccc1</smiles>

From isolated redox-active ester: Following General Procedure B, with SI-5 (29 mg, $0.1 \mathrm{mmol}$ ), Fe(acac) 3 (3.5 mg, $0.01 \mathrm{mmol}), \mathrm{dppBz}$ (5.4 mg, 0.012 $\mathrm{mmol}), \mathrm{Ph}_{2} \mathrm{Zn}(0.15 \mathrm{mmol})$ at $25{ }^{\circ} \mathrm{C}$. Product 9 was isolated by PTLC (pentane) to afford $12 \mathrm{mg}(68 \%)$ as colorless oil (note: volatile product).

$\mathbf{R}_{f}=0.76$ (hexanes).

${ }^{1} \mathrm{H}$ NMR (600 MHz, $\left.\mathrm{CDCl}_{3}\right): \delta 7.28(\mathrm{t}, J=7.6 \mathrm{~Hz}, 2 \mathrm{H}), 7.22-7.15(\mathrm{~m}, 1 \mathrm{H})$, $7.15-7.07(\mathrm{~m}, 2 \mathrm{H}), 2.39(\mathrm{tt}, J=9.5,5.3 \mathrm{~Hz}, 1 \mathrm{H}), 1.75-1.59(\mathrm{~m}, 2 \mathrm{H}), 1.59-$ $1.46(\mathrm{~m}, 2 \mathrm{H}), 1.33-1.00(\mathrm{~m}, 4 \mathrm{H}), 0.83(\mathrm{t}, J=7.3 \mathrm{~Hz}, 3 \mathrm{H}), 0.77(\mathrm{t}, J=7.4 \mathrm{~Hz}$, $3 \mathrm{H})$.

${ }^{13} \mathrm{C}$ NMR (151 MHz, $\left.\mathrm{CDCl}_{3}\right): \delta 146.5,128.5,128.1,126.1,48.2,36.61,30.2$, 30.1, 23.2, 14.4, 12.6.

Spectral data are in accordance with those reported in the literature.1

1-(Heptan-3-yl)-3-methoxybenzene (10).

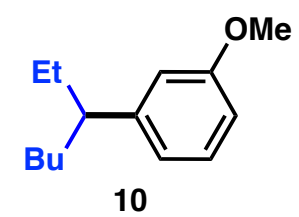

Following the General Procedure B with SI-5 (29 mg, $0.1 \mathrm{mmol})$, Fe $(\mathrm{acac})_{3}$ (3.5 mg, $0.01 \mathrm{mmol}), \mathrm{dppBz}$ (5.4 mg, $0.012 \mathrm{mmol}),(3-\mathrm{OMePh})_{2} \mathrm{Zn} \mathrm{(0.15}$ $\mathrm{mmol}$ ) at $25^{\circ} \mathrm{C}$. Product 10 was isolated by PTLC (hexanes/ethyl acetate 9:1) to afford $12.2 \mathrm{mg}(59 \%)$ as a transparent oil.

$\mathbf{R}_{f}=0.33$ (0.5:99.5 acetone/hexanes).

${ }^{1} \mathrm{H}$ NMR $\left(600 \mathrm{MHz}, \mathrm{CDCl}_{3}\right): \delta 7.20(\mathrm{t}, J=7.8 \mathrm{~Hz}, 1 \mathrm{H}), 6.82-6.71(\mathrm{~m}, 2 \mathrm{H})$, $6.69(\mathrm{~d}, J=1.9 \mathrm{~Hz}, 1 \mathrm{H}), 2.36(\mathrm{tt}, J=9.6,5.3 \mathrm{~Hz}, 1 \mathrm{H}), 1.73-1.58(\mathrm{~m}, 2 \mathrm{H})$, $1.57-1.49(\mathrm{~m}, 2 \mathrm{H}), 1.39-1.19(\mathrm{~m}, 2 \mathrm{H}), 1.20-1.01(\mathrm{~m}, 2 \mathrm{H}), 0.83(\mathrm{t}, J=7.3$ $\mathrm{Hz}, 3 \mathrm{H}), 0.77(\mathrm{t}, J=7.4 \mathrm{~Hz}, 3 \mathrm{H})$. 
${ }^{13} \mathrm{C}$ NMR (151 MHz, $\left.\mathrm{CDCl}_{3}\right): \delta 159.6,148.1,129.1,120.5,113.9,110.7,55.2$, 48.1, 36.4, 30.0, 29.8, 23.0, 14.2, 12.4 .

Spectral data are in accordance with those reported in the literature. ${ }^{1}$

Tert-butyl 4-phenylpiperidine-1-carboxylate (11).

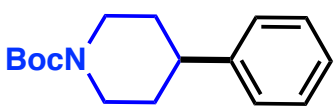

11

From isolated redox-active ester: Following General Procedure B, with SI-6 (37 mg, $0.1 \mathrm{mmol}), \mathrm{Fe}(\mathrm{acac})_{3}(3.5 \mathrm{mg}, 0.01 \mathrm{mmol}), \mathrm{dppBz}(5.4 \mathrm{mg}, 0.012$ $\mathrm{mmol}), \mathrm{Ph}_{2} \mathrm{Zn}(0.15 \mathrm{mmol})$ at $25{ }^{\circ} \mathrm{C}$. Product 11 was isolated by PTLC (hexanes:ethyl acetate 8:2) to afford $17 \mathrm{mg}(67 \%)$ as a colorless oil.

$\mathbf{R}_{f}=0.55$ (6:1 hexanes:ethyl acetate).

${ }^{1} \mathrm{H}$ NMR (400 MHz, $\left.\mathrm{CDCl}_{3}\right)$ : $\delta 7.32-7.30(\mathrm{~m}, 2 \mathrm{H}), 7.22-7.20(\mathrm{~m}, 3 \mathrm{H}), 4.24$ (d, $J=13.3 \mathrm{~Hz}, 2 \mathrm{H}), 2.85-2.76(\mathrm{~m}, 2 \mathrm{H}), 2.64(\mathrm{tt}, J=12.2,3.6 \mathrm{~Hz}, 1 \mathrm{H}), 1.89$ $-1.78(\mathrm{~m}, 2 \mathrm{H}), 1.67-1.58(\mathrm{~m}, 2 \mathrm{H}), 1.48(\mathrm{~s}, 9 \mathrm{H})$.

${ }^{13} \mathrm{C}$ NMR (101 MHz, $\left.\mathrm{CDCl}_{3}\right): \delta 155.0,146.0,128.7,126.9,126.5,79.6,44.6$, 42.9, 33.3, 28.7.

Spectral data are in accordance with those reported in the literature. ${ }^{1}$

4-Phenyltetrahydro-2H-pyran (12).

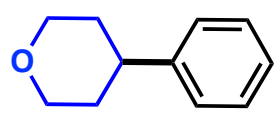

12

From isolated redox-active ester: Following General Procedure B, with SI-7 (28 mg, $0.1 \mathrm{mmol}), \mathrm{Fe}(\mathrm{acac})_{3}(3.5 \mathrm{mg}, 0.01 \mathrm{mmol}), \mathrm{dppBz}(5.4 \mathrm{mg}, 0.012$ $\mathrm{mmol}), \mathrm{Ph}_{2} \mathrm{Zn}(0.15 \mathrm{mmol})$ at $25^{\circ} \mathrm{C}$. Product 12 was isolated by PTLC (hexanes:ethyl acetate 8:2) to afford $10 \mathrm{mg}(63 \%)$ as a yellowish oil.

$\mathbf{R}_{f}=0.53$ (10:1 hexanes:ethyl acetate).

${ }^{1} \mathrm{H}$ NMR (600 MHz, $\left.\mathrm{CDCl}_{3}\right): \delta 7.34-7.31(\mathrm{~m}, 2 \mathrm{H}), 7.24-7.21(\mathrm{~m}, 3 \mathrm{H}), 4.09$ (dd, $J=11.3,4.0 \mathrm{~Hz}, 2 \mathrm{H}), 3.54(\mathrm{td}, J=11.7,2.0 \mathrm{~Hz}, 2 \mathrm{H}), 2.79-2.73(\mathrm{~m}, 1 \mathrm{H})$, $1.91-1.71(\mathrm{~m}, 4 \mathrm{H})$;

${ }^{13} \mathrm{C}$ NMR (151 MHz, $\left.\mathrm{CDCl}_{3}\right): \delta 146.0,128.7,126.9,126.5,68.6,41.7,34.1$. 
Spectral data are in accordance with those reported in the literature. ${ }^{1}$

\section{4-Phenyl-1-tosylpiperidine (13).}

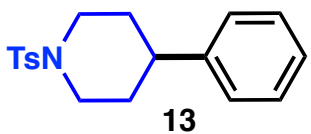

From isolated redox-active ester. Following General Procedure B, with SI-13 (43 mg, $0.1 \mathrm{mmol}), \mathrm{Fe}(\mathrm{acac})_{3}(3.5 \mathrm{mg}, 0.01 \mathrm{mmol}), \mathrm{dppBz}(5.4 \mathrm{mg}, 0.012$ $\mathrm{mmol}), \mathrm{Ph}_{2} \mathrm{Zn}(0.15 \mathrm{mmol})$ at $25{ }^{\circ} \mathrm{C}$. Product 13 was isolated by Column chromatography (hexanes:ethyl acetate 9:1) to afford $24 \mathrm{mg}(76 \%)$ as a white solid.

From redox-active ester generated in situ with HATU: Following General Procedure $\mathrm{C}$, with $\mathrm{N}$-tosylpiperidine-4-carboxylic acid 46 (28 $\mathrm{mg}, 0.1 \mathrm{mmol})$, HATU (38 mg, $0.1 \mathrm{mmol}), \mathrm{Et}_{3} \mathrm{~N}$ (14 $\left.\mu \mathrm{L}, 0.1 \mathrm{mmol}\right), \mathrm{Fe}(\mathrm{acac})_{3}(3.5 \mathrm{mg}, 0.01$ $\mathrm{mmol}), \mathrm{dppBz}(8.9 \mathrm{mg}, 0.02 \mathrm{mmol}), \mathrm{Ph}_{2} \mathrm{Zn}(0.25 \mathrm{mmol})$ at $25^{\circ} \mathrm{C}$. Product 13 was isolated by column chromatography (hexanes:ethyl acetate 9:1) to afford $24 \mathrm{mg}(76 \%)$ as a white solid.

$\mathbf{R}_{\boldsymbol{f}}=0.51$ (hexanes:ethyl acetate $3: 1$ )

${ }^{1} \mathrm{H}$ NMR (600 MHz, $\left.\mathrm{CDCl}_{3}\right) \delta 7.71-7.66(\mathrm{~m}, 2 \mathrm{H}), 7.38-7.32(\mathrm{~m}, 2 \mathrm{H}), 7.32-$ $7.27(\mathrm{~m}, 2 \mathrm{H}), 7.23-7.19(\mathrm{~m}, 1 \mathrm{H}), 7.17-7.12(\mathrm{~m}, 2 \mathrm{H}), 3.96-3.90(\mathrm{~m}, 2 \mathrm{H})$, $2.45(\mathrm{~s}, 3 \mathrm{H}), 2.41$ (tt, $J=11.6,4.2 \mathrm{~Hz}, 1 \mathrm{H}), 2.34(\mathrm{td}, J=11.8,3.3 \mathrm{~Hz}, 2 \mathrm{H})$, $1.92-1.79(\mathrm{~m}, 4 \mathrm{H})$.

${ }^{13} \mathrm{C}$ NMR $\left(151 \mathrm{MHz}, \mathrm{CDCl}_{3}\right) \delta 145.0,143.6,133.3,129.8,128.7,127.9$, 126.8, 126.7, 47.0, 42.0, 32.7, 21.7.

Spectral data are in accordance with those reported in the literature. ${ }^{7}$

\section{2-Phenyltetrahydrofuran (14).}

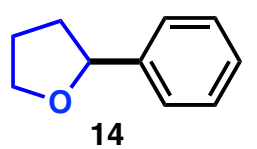

From isolated redox-active ester: Following General Procedure B, with SI-8 (26 mg, $0.1 \mathrm{mmol}), \mathrm{Fe}(\mathrm{acac})_{3}(3.5 \mathrm{mg}, 0.01 \mathrm{mmol}), \mathrm{dppBz}(5.4 \mathrm{mg}, 0.012$ 
$\mathrm{mmol}), \mathrm{Ph}_{2} \mathrm{Zn}(0.15 \mathrm{mmol})$ at $25{ }^{\circ} \mathrm{C}$. Product 14 was isolated by PTLC (hexanes:ethyl acetate 8:2) to afford $11 \mathrm{mg}(68 \%)$ as a yellowish oil. $\boldsymbol{R}_{\boldsymbol{f}}=0.53$ (10:1 hexanes:ethyl acetate).

${ }^{1} \mathrm{H}$ NMR (600 MHz, $\left.\mathrm{CDCl}_{3}\right): \delta 7.35-7.32(\mathrm{~m}, 4 \mathrm{H}), 7.26-7.24(\mathrm{~m}, 1 \mathrm{H}), 4.90$ (t, $J=7.2, \mathrm{~Hz}, 1 \mathrm{H}), 4.20-4.05(\mathrm{~m}, 1 \mathrm{H}), 3.94(\mathrm{td}, J=7.9,6.4 \mathrm{~Hz}, 1 \mathrm{H}), 2.33$ (ddd, $J=12.4,7.2,5.5 \mathrm{~Hz}, 1 \mathrm{H}), 2.07-1.94(\mathrm{~m}, 2 \mathrm{H}), 1.81$ (dq, $J=12.3,7.7$ $\mathrm{Hz}, 1 \mathrm{H})$.

${ }^{13} \mathrm{C}$ NMR (151 MHz, $\left.\mathrm{CDCl}_{3}\right): \delta 143.6,128.4,127.2,125.8,80.8,68.8,34.8$, and 26.2 .

Spectral data are in accordance with those reported in the literature.1

\section{Tert-butyl 2-phenylpyrrolidine-1-carboxylate (15).}<smiles>CC(C)(C)[N+]1CCCC1c1ccccc1</smiles>

From isolated redox-active ester. Following General Procedure B, with SI-27 (36 mg, $0.1 \mathrm{mmol}), \mathrm{FeCl}_{3}(0.8 \mathrm{mg}, 0.005 \mathrm{mmol}), \mathrm{dppBz}(2.7 \mathrm{mg}, 0.006 \mathrm{mmol})$, $\mathrm{Ph}_{2} \mathrm{Zn}(0.15 \mathrm{mmol})$ at $25^{\circ} \mathrm{C}$. Product 15 was isolated by PTLC (hexanes:ethyl acetate $20: 3)$ to afford $15.2 \mathrm{mg}(62 \%)$ as a colorless oil.

$\mathbf{R}_{f}=0.53$ (20:3 hexanes:ethyl acetate).

The product gives two sets of NMR signals, owing to the presence of rotamers.

${ }^{1} \mathrm{H}$ NMR (600 MHz, $\left.\mathbf{C D C l}_{3}\right): \delta 7.27-7.23(\mathrm{~m}, 2 \mathrm{H}), 7.19-7.13(\mathrm{~m}, 3 \mathrm{H}), 4.93$ (brs, 0.3H), 4.73 (brs, 0.7H), $3.60-3.49(\mathrm{~m}, 2 \mathrm{H}), 2.30$ (br, 1H), $1.89(\mathrm{br}, 1 \mathrm{H})$, $1.85-1.75(\mathrm{~m}, 2 \mathrm{H}), 1.43(\mathrm{~s}, 3 \mathrm{H}), 1.15(\mathrm{~s}, 6 \mathrm{H})$.

${ }^{13} \mathrm{C}$ NMR (151 MHz, $\mathbf{C D C l}_{3}$ ): $\delta$ 154.7, 145.3, 144.2, 128.4, 128.2, 126.6, 125.6, 125.5, 79.3, 61.5, 60.8, 47.5, 47.2, 36.2, 35.0, 28.7, 28.3, 23.6, 23.3. Spectral data are in accordance with those reported in the literature.1 
3-(Heptane-3-yl)pyridine (16).

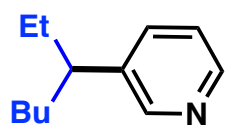

16

Following the General Procedure B with SI-5 (29 mg, $0.1 \mathrm{mmol})$, Fe(acac) 3 (3.5 mg, $0.01 \mathrm{mmol}), \mathrm{dppBz}$ (5.4 mg, $0.012 \mathrm{mmol}),(3-p y r i d y l){ }_{2} Z \mathrm{n}(0.15 \mathrm{mmol})$ at $25{ }^{\circ} \mathrm{C}$. Product 16 was isolated by PTLC (hexanes:ethyl acetate 5:1) to afford $8.4 \mathrm{mg}(44 \%)$ as a transparent oil.

$\mathbf{R}_{f}=0.50$ (hexanes:ethyl acetate 5:1).

${ }^{1} \mathrm{H}$ NMR (400 MHz, $\left.\mathrm{CDCl}_{3}\right): \delta 8.43(\mathrm{dd}, J=4.7,1.7 \mathrm{~Hz}, 1 \mathrm{H}), 8.41-8.37(\mathrm{~m}$, 1H), 7.45 (dt, $J=7.8,2.0 \mathrm{~Hz}, 1 \mathrm{H}$ ), 7.22 (ddd, $J=7.9,4.8,0.9 \mathrm{~Hz}, 1 \mathrm{H}$ ), 2.41 (td, $J=9.3,4.8 \mathrm{~Hz}, 1 \mathrm{H}$ ), 1.69 (dddt, $J=21.6,13.5,10.7,5.5 \mathrm{~Hz}, 2 \mathrm{H}$ ), $1.59-$ $1.49(\mathrm{~m}, 2 \mathrm{H}), 1.32-1.19(\mathrm{~m}, 2 \mathrm{H}), 1.19-1.11(\mathrm{~m}, 1 \mathrm{H}), 1.10-1.03(\mathrm{~m}, 1 \mathrm{H})$, $0.82(\mathrm{t}, J=7.3 \mathrm{~Hz}, 3 \mathrm{H}), 0.76(\mathrm{t}, J=7.4 \mathrm{~Hz}, 3 \mathrm{H})$.

${ }^{13} \mathrm{C}$ NMR (151 MHz, $\left.\mathrm{CDCl}_{3}\right): \delta 150.0,147.5,141.3,134.9,123.5,45.4,36.0$, 29.8, 29.6, 22.8, 14.1, 12.2.

Spectral data are in accordance with those reported in the literature.1

\section{1,4-Diphenylbutane (17).}<smiles>[Te]CCCc1ccccc1</smiles>

From isolated redox-active ester: Following General Procedure B, with SI-9 (47.5 mg, $0.1 \mathrm{mmol}), \mathrm{FeCl}_{3}(1.6 \mathrm{mg}, 0.01 \mathrm{mmol}), \mathrm{dppBz}$ (5.4 mg, 0.012 $\mathrm{mmol}), \mathrm{Ph}_{2} \mathrm{Zn}(0.15 \mathrm{mmol})$ at $25^{\circ} \mathrm{C}$. Product 17 was isolated by PTLC (hexanes) to afford $14.1 \mathrm{mg}(67 \%)$ as yellowish oil.

Kumada-coupling using Fe-catalysis from isolated redox-active ester: with SI35 (32 mg, $0.1 \mathrm{mmol}), \mathrm{Fe}(\mathrm{acac})_{3}(7 \mathrm{mg}, 0.02 \mathrm{mmol}), \mathrm{PhMgBr} \cdot \mathrm{LiCl}(0.2 \mathrm{mmol})$ at $0^{\circ} \mathrm{C}$. The crude product was purified on silica gel (hexanes) resulting in an 
inseparable mixture $(17.8 \mathrm{mg}$ ) of biphenyl and product 17. Percent yield was calculated by NMR resulting in a $57.9 \mathrm{wt} \%$ mixture of $\mathbf{1 7}(10.3 \mathrm{mg}, 49 \%$ yield).

Negishi-coupling using Ni-catalysis. Following General Procedure E, with SI-9 (47.5 mg, $0.1 \mathrm{mmol}$ ), $\mathrm{NiCl}_{2} \cdot$ glyme (4.4 mg, $0.02 \mathrm{mmol}$ ), di-tert-butylbipyridine $(11 \mathrm{mg}, 0.04 \mathrm{mmol}), \mathrm{PhZnCl}(0.3 \mathrm{mmol})$ at $25^{\circ} \mathrm{C}$. Product 17 was isolated by PTLC (hexanes) to afford $14.3 \mathrm{mg}(68 \%)$ as yellowish oil.

$\mathbf{R}_{f}=0.90$ (hexanes).

${ }^{1} \mathrm{H}$ NMR (600 MHz, $\left.\mathrm{CDCl}_{3}\right): \delta 7.30-7.27(\mathrm{~m}, 4 \mathrm{H}), 7.20-7.17(\mathrm{~m}, 6 \mathrm{H}), 2.67-$ $2.64(\mathrm{~m}, 4 \mathrm{H}), 1.72-1.65(\mathrm{~m}, 4 \mathrm{H})$.

${ }^{13} \mathrm{C}$ NMR (151 MHz, $\left.\mathrm{CDCl}_{3}\right): \delta 142.7,128.6,128.4,125.8,36.0,31.2$.

Spectral data are in accordance with those reported in the literature. ${ }^{8}$

Methyl 5-phenylpentanoate (18).<smiles>CC(=O)CCCCc1ccccc1</smiles>

From isolated redox-active ester. Following General Procedure B, with SI-10 (44.3 mg, $0.1 \mathrm{mmol}), \mathrm{FeCl}_{3}(0.8 \mathrm{mg}, 0.005 \mathrm{mmol}), \mathrm{dppBz}(2.7 \mathrm{mg}, 0.006$ $\mathrm{mmol}), \mathrm{Ph}_{2} \mathrm{Zn}(0.15 \mathrm{mmol})$ at $0{ }^{\circ} \mathrm{C}$. Product 18 was isolated by PTLC (hexanes:ethyl acetate $12: 1)$ to afford $12.1 \mathrm{mg}(63 \%)$ as a yellowish oil.

Negishi-coupling using Ni-catalysis. Following General Procedure E, with SI10 (44.3 mg, $0.1 \mathrm{mmol}$ ), $\mathrm{NiCl}_{2} \cdot$ glyme $(4.4 \mathrm{mg}, 0.02 \mathrm{mmol}$ ), di-tertbutylbipyridine (11 mg, $0.04 \mathrm{mmol}), \mathrm{PhZnCl}(0.3 \mathrm{mmol})$ at $25^{\circ} \mathrm{C}$. Product 18 was isolated by PTLC (hexanes) to afford $9.7 \mathrm{mg}(51 \%)$ as yellowish oil.

$\mathbf{R}_{f}=0.50$ (hexanes:ethyl acetate 12:1).

${ }^{1} \mathrm{H}$ NMR (600 MHz, $\left.\mathrm{CDCl}_{3}\right)$ : $\delta 7.29-7.27(\mathrm{~m}, 2 \mathrm{H}), 7.19-7.17(\mathrm{~m}, 3 \mathrm{H}), 3.67$ (s, 3H), $2.63(\mathrm{t}, J=7.2 \mathrm{~Hz}, 2 \mathrm{H}), 2.34(\mathrm{t}, J=7.2 \mathrm{~Hz}, 2 \mathrm{H}), 1.71-1.63(\mathrm{~m}, 4 \mathrm{H})$. 
${ }^{13} \mathrm{C}$ NMR (151 MHz, $\left.\mathrm{CDCl}_{3}\right): \delta$ 174.2, 142.3, 128.5, 128.5, 125.9, 51.6, 35.7, 34.1, 31.0, 24.7.

Spectral data are in accordance with those reported in the literature. ${ }^{9}$

Tert-butyldimethyl(2-phenylpropoxy)silane (19).<smiles>CCCCC([N])c1ccccc1</smiles>

From isolated redox-active ester. Following General Procedure B, with SI-11 (36 mg, $0.1 \mathrm{mmol}), \mathrm{Fe}(\mathrm{acac})_{3}(3.5 \mathrm{mg}, 0.01 \mathrm{mmol}), \mathrm{dppBz}(5.4 \mathrm{mg}, 0.012$ $\mathrm{mmol}), \mathrm{Ph}_{2} \mathrm{Zn}(0.15 \mathrm{mmol})$ at $25^{\circ} \mathrm{C}$. Product 19 was isolated by PTLC (hexanes:ethyl acetate 9:1) to afford $16 \mathrm{mg} \mathrm{(64 \% )}$ as yellowish oil.

$\mathbf{R}_{f}=0.33$ (9:1 hexanes:ethyl acetate).

${ }^{1} \mathrm{H}$ NMR (600 MHz, $\left.\mathrm{CDCl}_{3}\right): \delta 7.31-7.27(\mathrm{~m}, 2 \mathrm{H}), 7.24-7.18(\mathrm{~m}, 3 \mathrm{H}), 3.69$ (dd, $J=9.8,5.9 \mathrm{~Hz}, 1 \mathrm{H}$ ), 3.59 (dd, $J=9.8,7.6 \mathrm{~Hz}, 1 \mathrm{H}), 2.94-2.84(\mathrm{~m}, 1 \mathrm{H})$, 1.29 (d, J=7.0 Hz, 3H), 0.86 (s, 9H), -0.03 (s, 3H), -0.04 (s, 3H).

${ }^{13}$ C NMR (151 MHz, $\left.\mathrm{CDCl}_{3}\right): \delta 144.7,128.3,127.7,126.4,69.4,42.6,26.1$, $18.5,17.6,-5.30,-5.33$.

Spectral data are in accordance with those reported in the literature. 1

\section{Methyl-3-phenylcyclopentane-1-carboxylate.}<smiles>CC(=O)C1CCC(c2ccccc2)C1</smiles>

20

From isolated redox-active ester. Following General Procedure B, with SI-28 (160 mg, $0.5 \mathrm{mmol}), \mathrm{Fe}(\mathrm{acac})_{3}$ (19 mg, $\left.0.054 \mathrm{mmol}\right), \mathrm{dppBz}$ (28 mg, 0.061 $\mathrm{mmol}), \mathrm{Ph}_{2} \mathrm{Zn}(1.5 \mathrm{mmol})$ at $25^{\circ} \mathrm{C}$ for 15 minutes. Upon concentration, a viscous, red oil was obtained with mass of $172 \mathrm{mg}$. Quantitative NMR of the oil $\left(\mathrm{CD}_{3} \mathrm{OD}\right.$, fumaric acid internal standard) revealed a potency of $38 \%$, corresponding to a yield of $63 \%(64.7 \mathrm{mg}, 0.317 \mathrm{mmol})$. The oil was purified by silica gel chromatography (hexanes:MTBE, $0-10 \%$ gradient); the desired product 20 eluted at $5-6 \%$ MTBE, and was concentrated to a colorless oil. 
Using Ni-catalysis. Following General Procedure E, with SI-28 $(100 \mathrm{mg}, 0.32$ $\mathrm{mmol}$ ), NiCl $2 \cdot$ glyme (14.1 mg, $0.063 \mathrm{mmol}$ ), di-tert-butylbipyridine $(35.3 \mathrm{mg}$, $0.13 \mathrm{mmol}), \mathrm{PhZnCl}(2.2 \mathrm{~mL}, 0.95 \mathrm{mmol}, 0.43 \mathrm{M})$ at $25{ }^{\circ} \mathrm{C}$. The oil was purified by silica gel chromatography (hexanes:MTBE, 0-10\% gradient); the desired product 20 eluted at 5-6\% MTBE, and was concentrated to a colorless oil of mass $34.2 \mathrm{mg}$ (53\% yield). The diastereoselectivity, as determined by NMR, was 1.3:1.

$\mathbf{R}_{\boldsymbol{f}}=0.75$ (silica gel, 2:1 heptane:MTBE);

${ }^{1} \mathbf{H}$ NMR (500 MHz, $\left.\mathrm{CDCl}_{3}\right) \delta 7.36-7.19(\mathrm{~m}, 5 \mathrm{H}), 3.74(\mathrm{~s}, 3 \mathrm{H}), 3.29-3.21$ (trans diastereomer, $\mathrm{m}, 1 \mathrm{H}$ ), $3.10-3.02(\mathrm{~m}, 1 \mathrm{H}), 3.01-2.93$ (cis diastereomer, $\mathrm{m}, 1 \mathrm{H}), 2.44-2.36(\mathrm{~m}, 1 \mathrm{H}), 2.26-2.09(\mathrm{~m}, 2 \mathrm{H}), 2.09-1.90$ $(\mathrm{m}, 2 \mathrm{H}), 1.90-1.78$ (cis diastereomer, $\mathrm{m}, 1 \mathrm{H}$ ), $1.78-1.66$ (trans diastereomer, $\mathrm{m}, 1 \mathrm{H})$.

${ }^{13} \mathrm{C}$ NMR (126 MHz, $\left.\mathrm{CDCl}_{3}\right) \delta 177.2,145.0,128.4,127.0,126.1,51.8,44.9$, 43.0, 37.5, 34.6, 30.0.

Spectral data are in accordance with those reported in the literature. ${ }^{10}$

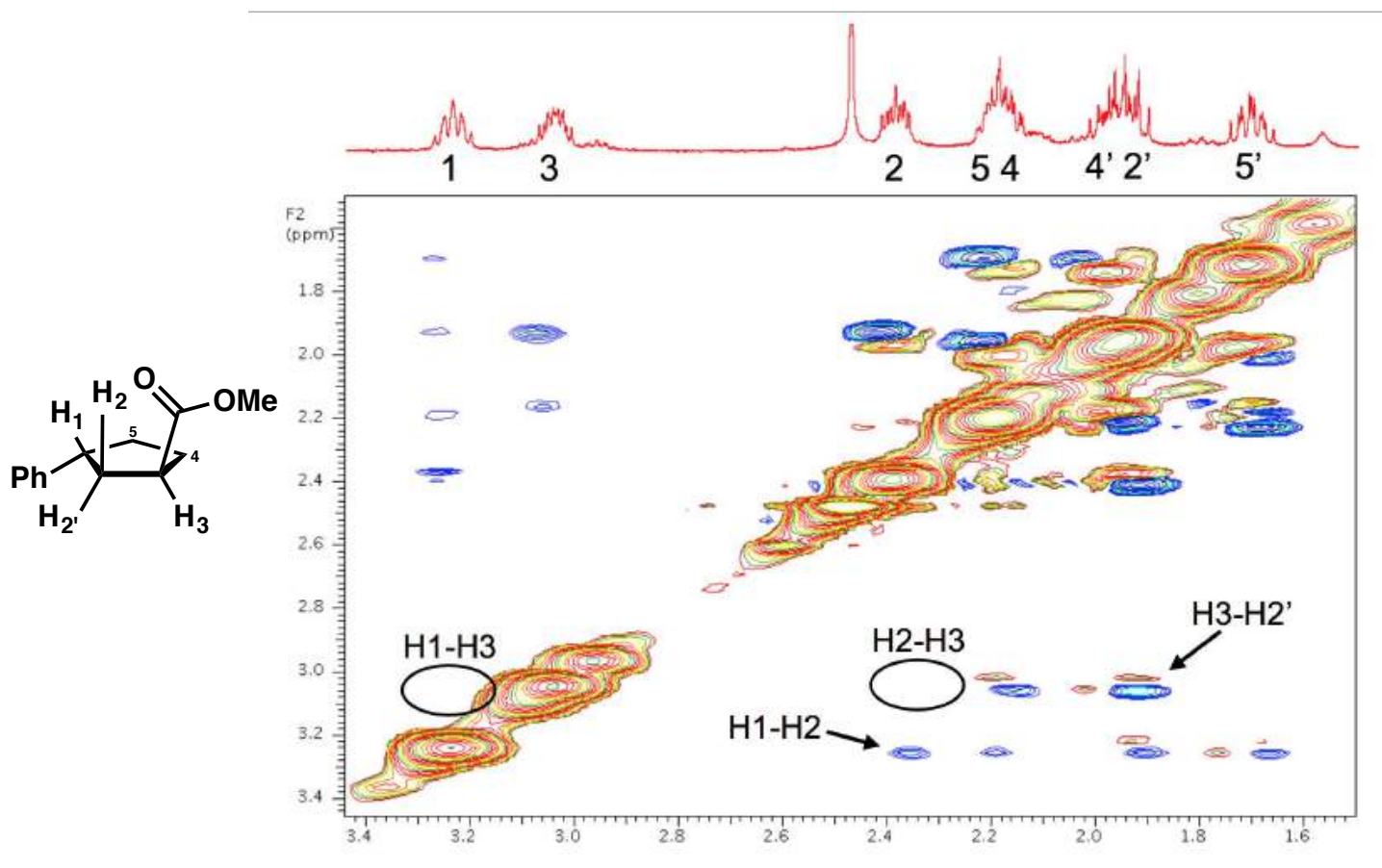


The ROESY spectrum then was able to observe NOE transfer between protons $\mathrm{H} 1$ and $\mathrm{H} 2, \mathrm{H} 3$ and $\mathrm{H} 2$ '. Finally, NOE transfer was observed between neither $\mathrm{H} 1$ and $\mathrm{H} 3$, nor $\mathrm{H} 2$ and $\mathrm{H} 3$, confirming a trans relationship between $\mathrm{H} 1$ and $\mathrm{H} 3$.

\section{1-CyclohexyInaphthalene (21).}<smiles>c1ccc2c(C3CCCCC3)cccc2c1</smiles>

From isolated redox-active ester: Following General Procedure B, with 1 (27 $\mathrm{mg}, 0.1 \mathrm{mmol}), \mathrm{Fe}(\mathrm{acac})_{3}(3.5 \mathrm{mg}, 0.01 \mathrm{mmol}), \mathrm{dppBz}(5.4 \mathrm{mg}, 0.012 \mathrm{mmol})$, THF $(0.5 \mathrm{~mL})$, and $\mathrm{Np}_{2} \mathrm{Zn}(0.15 \mathrm{mmol})$ at $25{ }^{\circ} \mathrm{C}$. The crude white solid was purified on silica gel (heptanes) resulting in an inseparable mixture $(26.8 \mathrm{mg})$ of naphthalene and product 21. Percent yield was calculated by QNMR using trimethoxybenzene as the internal standard resulting in a $46.33 \mathrm{wt} \%$ mixture of 21 (12.4 mg, 60\% yield).

Kumada-coupling using $\mathrm{Fe}$-catalysis from isolated redox-active ester. Following General Procedure D, with 1 (27 mg, $0.1 \mathrm{mmol}), \mathrm{Fe}(\mathrm{acac})_{3}(7.1 \mathrm{mg}$, $0.02 \mathrm{mmol}), 1-\mathrm{NpMgBr} \cdot \mathrm{LiCl}(0.88 \mathrm{M}, 0.3 \mathrm{mmol})$ at $0{ }^{\circ} \mathrm{C}$. Product 21 was isolated by PTLC (hexanes), and subsequent removal of naphthalene in vacuo to afford $12 \mathrm{mg}(57 \%)$ as a colorless oil.

$\mathbf{R}_{\boldsymbol{f}}=0.55$ (hexanes)

${ }^{1} \mathrm{H}$ NMR $\left(600 \mathrm{MHz}, \mathrm{CDCl}_{3}\right) \delta 8.13(\mathrm{~d}, J=8.5 \mathrm{~Hz}, 1 \mathrm{H}), 7.86(\mathrm{~d}, J=8.1 \mathrm{~Hz}$, $1 \mathrm{H}$ ), 7.70 (d, $J=8.0 \mathrm{~Hz}, 1 \mathrm{H}$ ), 7.51 (ddd, $J=8.5,6.8,1.5 \mathrm{~Hz}, 1 \mathrm{H}$ ), 7.47 (ddd, $J$ $=8.0,6.8,1.3 \mathrm{~Hz}, 1 \mathrm{H}), 7.45(\mathrm{~d}, 1 \mathrm{H}), 7.40(\mathrm{dd}, J=7.3,1.3 \mathrm{~Hz}, 1 \mathrm{H}), 3.34(\mathrm{tt}, J$ $=11.3,3.1 \mathrm{~Hz}, 1 \mathrm{H}), 2.09-2.01(\mathrm{~m}, 2 \mathrm{H}), 1.98-1.90(\mathrm{~m}, 2 \mathrm{H}), 1.88-1.83(\mathrm{~m}$, $1 \mathrm{H}), 1.62-1.52(\mathrm{~m}, 4 \mathrm{H}), 1.40-1.32(\mathrm{~m}, 1 \mathrm{H})$.

${ }^{13} \mathrm{C}$ NMR (151 MHz, $\left.\mathrm{CDCl}_{3}\right) \delta 144.0,134.1,131.5,129.1,126.3,125.8$, 125.7, 125.3, 123.3, 122.4, 39.4, 34.4, 27.5, 26.7. 
Spectral data are in accordance with those reported in the literature. ${ }^{11}$ 
(((1R,2S,5R)-2-Isopropyl-5-methylcyclohexyloxy)methyl)benzene (22).<smiles>CC(C)[C@H]1CC[C@@H](C)CC1OCc1ccccc1</smiles>

From isolated redox-active ester. Following General Procedure B, with SI-12 (49.7 mg, $0.1 \mathrm{mmol}), \mathrm{FeCl}_{3}(0.8 \mathrm{mg}, 0.005 \mathrm{mmol}), \mathrm{dppBz}(2.7 \mathrm{mg}, 0.006$ $\mathrm{mmol}), \mathrm{Ph}_{2} \mathrm{Zn}(0.15 \mathrm{mmol})$ at $0{ }^{\circ} \mathrm{C}$. Product 22 was isolated by PTLC (hexanes:ethyl acetate $15: 1)$ to afford $14.2 \mathrm{mg}(58 \%)$ as a yellowish oil.

Negishi-coupling using Ni-catalysis: Following General Procedure E, with SI12 (49.7 mg, $0.1 \mathrm{mmol}$ ), $\mathrm{NiCl}_{2} \cdot$ glyme $(4.4 \mathrm{mg}, 0.02 \mathrm{mmol})$, di-tertbutylbipyridine $(11 \mathrm{mg}, 0.04 \mathrm{mmol}), \mathrm{PhZnCl}(0.3 \mathrm{mmol})$ at $25^{\circ} \mathrm{C}$. Product 22 was isolated by PTLC (hexanes:ethyl acetate 15:1) to afford $12.6 \mathrm{mg}(51 \%)$ as a yellowish oil.

$\mathbf{R}_{f}=0.50$ (hexanes:ethyl acetate 12:1).

${ }^{1} \mathrm{H}$ NMR (600 MHz, $\left.\mathrm{CDCl}_{3}\right): \delta 7.37-7.32(\mathrm{~m}, 4 \mathrm{H}), 7.28-7.26(\mathrm{~m}, 1 \mathrm{H}), 4.66$ (d, $J=11.4 \mathrm{~Hz}, 2 \mathrm{H}$ ), 4.41 (d, $J=11.4 \mathrm{~Hz}, 2 \mathrm{H}), 3.18(\mathrm{td}, J=10.8,4.2 \mathrm{~Hz}, 1 \mathrm{H}$ ), $2.34-2.28(\mathrm{~m}, 1 \mathrm{H}), 2.20-2.18(\mathrm{~m}, 1 \mathrm{H}), 1.69-1.61(\mathrm{~m}, 1 \mathrm{H}), 1.41-1.34(\mathrm{~m}$, $1 \mathrm{H}), 1.33-1.28(\mathrm{~m}, 1 \mathrm{H}), 1.02-0.83(\mathrm{~m}, 10 \mathrm{H}), 0.72(\mathrm{~d}, J=6.6 \mathrm{~Hz}, 3 \mathrm{H})$.

${ }^{13} \mathrm{C}$ NMR (151 MHz, $\left.\mathrm{CDCl}_{3}\right)$ : $\delta 139.3,128.4,128.0,127.6,78.9,70.6,48.5$, $40.5,34.7,31.7,25.7,23.4,22.5,21.2,16.2$.

Spectral data are in accordance with those reported in the literature. ${ }^{12}$ 


\section{3-Phenethylpyridine (23).}

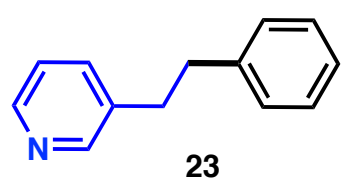

From in situ generation of redox-active ester: Following the General Procedure C with 3-pyridylpropionic acid (15 mg, $0.1 \mathrm{mmol}$ ), HATU (38 mg), $\mathrm{Et}_{3} \mathrm{~N}(14 \mu \mathrm{L}), \mathrm{Fe}(\mathrm{acac})_{3}(3.5 \mathrm{mg}, 0.01 \mathrm{mmol}), \mathrm{dppBz}(8.9 \mathrm{mg}, 0.02 \mathrm{mmol})$, $\mathrm{Ph}_{2} \mathrm{Zn}(0.25 \mathrm{mmol})$ at $25^{\circ} \mathrm{C}$. Product 23 was isolated by PTLC (hexanes:ethyl acetate $1: 1)$ to afford $13.1 \mathrm{mg}(72 \%)$ as a transparent oil.

$1.0 \mathrm{Mmol}$ scale from in situ generation of redox-active ester: Following the General Procedure C with 3-pyridylpropionic acid (150 mg, $1.0 \mathrm{mmol}$ ), HATU (380 mg), Et $\mathrm{E}_{3} \mathrm{~N}$ (140 $\left.\mu \mathrm{L}\right), \mathrm{Fe}(\mathrm{acac})_{3}$ (35 mg, $\left.0.1 \mathrm{mmol}\right), \mathrm{dppBz}$ (89 mg, 0.2 $\mathrm{mmol}), \mathrm{Ph}_{2} \mathrm{Zn}(2.5 \mathrm{mmol})$ at $25^{\circ} \mathrm{C}$. Product 23 was isolated by column chromatography (hexanes:ethyl acetate 1:1) to afford $128 \mathrm{mg}(70 \%)$ as a transparent oil.

Using Ni-catalysis and in situ generation of redox-active ester. Following General Procedure F, with 3-pyridylpropionic acid (15 mg, $0.1 \mathrm{mmol}$ ), HATU (38 mg), $\mathrm{Et}_{3} \mathrm{~N}(14 \mu \mathrm{L}), \mathrm{NiCl}_{2} \cdot g l y m e ~(4.4 \mathrm{mg}, 0.02 \mathrm{mmol})$, di-tert-butylbipyridine (11 mg, $0.04 \mathrm{mmol}), \mathrm{PhZnCl}(0.3 \mathrm{mmol})$ at $25^{\circ} \mathrm{C}$. Product 23 was isolated by PTLC (hexanes:EtOAc 1:1) to afford $6.0 \mathrm{mg}(33 \%)$ as a transparent oil.

$\mathbf{R}_{\boldsymbol{f}}=0.43$ (hexanes:ethylacetate $1: 1$ )

${ }^{1} \mathrm{H}$ NMR (600 MHz, $\mathrm{CDCl}_{3}$ ) $\delta 8.47$ (dd, $\left.J=4.8,1.6 \mathrm{~Hz}, 2 \mathrm{H}\right), 8.45$ (d, $J=2.3$ $\mathrm{Hz}, 1 \mathrm{H}), 7.33-7.29(\mathrm{~m}, 2 \mathrm{H}), 7.25-7.20(\mathrm{~m}, 2 \mathrm{H}), 7.17(\mathrm{dd}, J=8.0,1.3 \mathrm{~Hz}$, $2 \mathrm{H}), 2.95(\mathrm{~s}, 4 \mathrm{H})$.

${ }^{13} \mathrm{C}$ NMR (151 MHz, $\left.\mathbf{C D C l}_{3}\right) \delta 149.5,147.0,140.3,136.4,135.5,128.0$, 127.9, 125.7, 122.8, 37.00, 34.5.

Spectral data are in accordance with those reported in the literature. ${ }^{13}$ 
Tert-butyl 4-(3-(benzyloxy)propyl)piperidine-1-carboxylate (24).<smiles>CC(C)(C)ON1CCC(CCCc2ccccc2)CC1</smiles>

From isolated redox-active ester. Following General Procedure B, with SI-23 (51.2 mg, $0.1 \mathrm{mmol}), \mathrm{Fe}(\mathrm{acac})_{3}(7.1 \mathrm{mg}, 0.020 \mathrm{mmol}), \mathrm{dppBz}(10.7 \mathrm{mg}, 0.024$ $\mathrm{mmol}),\left(\mathrm{PhCH}_{2} \mathrm{CH}_{2} \mathrm{CH}_{2}\right)_{2} \mathrm{Zn}(0.15 \mathrm{mmol})$ at r.t. Product 24 was isolated by PTLC (hexanes:ethyl acetate 5:1) to afford $16.3 \mathrm{mg}(55 \%)$ as yellowish oil. $\mathbf{R}_{\boldsymbol{f}}=0.30$ (hexanes:ethyl acetate 5:1).

${ }^{1} \mathrm{H}$ NMR (500 MHz, $\mathbf{C D C l}_{3}$ ): $\delta$ 7.29-7.26 (m, 2H), 7.19-7.16 (m, 3H), 4.05 (br, 2H), $2.66(\mathrm{br}, 2 \mathrm{H}), 2.60$ (t, $J=7.8 \mathrm{~Hz}, 2 \mathrm{H}), 1.66-1.61(\mathrm{~m}, 4 \mathrm{H}), 1.45(\mathrm{~s}, 9 \mathrm{H})$, 1.41-1.35 (m, 1H), 1.30-1.26 (m, 2H), 1.10-1.03 (m, 2H).

${ }^{13} \mathrm{C}$ NMR (151 MHz, $\left.\mathrm{CDCl}_{3}\right): \delta$ 155.1, 142.7, 128.5, 128.4, 125.8, 79.3, 44.3, $36.3,36.2,36.1,32.3,28.7,28.6$.

HRMS (ESI-TOF): calc'd for $\mathrm{C}_{19} \mathrm{H}_{30} \mathrm{NO}_{2}[\mathrm{M}+\mathrm{H}]^{+}$304.2271; found 304.2271.

4-(3-(Benzyloxy)propyl)tetrahydro-2 H-pyran (25).<smiles>c1ccc(CCCC2CCOCC2)cc1</smiles>

From isolated redox-active ester. Following General Procedure B, with SI-24 (41.3 mg, $0.1 \mathrm{mmol}), \mathrm{Fe}(\mathrm{acac})_{3}(7.1 \mathrm{mg}, 0.020 \mathrm{mmol})$, dppBz (10.7 mg, 0.024 $\mathrm{mmol}),\left(\mathrm{BnOCH}_{2} \mathrm{CH}_{2} \mathrm{CH}_{2}\right)_{2} \mathrm{Zn}(0.15 \mathrm{mmol})$ at r.t.. Product 25 was isolated by PTLC (hexanes:ethyl acetate 8:1) to afford $11.0 \mathrm{mg} \mathrm{(54 \% )} \mathrm{as} \mathrm{a} \mathrm{yellowish} \mathrm{oil.}$ $\mathbf{R}_{\boldsymbol{f}}=0.30$ (hexanes:ethyl acetate 8:1).

${ }^{1} \mathrm{H}$ NMR (600 MHz, $\left.\mathbf{C D C l}_{3}\right)$ : $\delta 7.29-7.27(\mathrm{~m}, 2 \mathrm{H}), 7.20-7.17(\mathrm{~m}, 3 \mathrm{H}), 3.94$ (dd, $J=11.4,4.8 \mathrm{~Hz}, 2 \mathrm{H}), 3.36$ (td, $J=12.0,1.8 \mathrm{~Hz}, 2 \mathrm{H}), 2.60$ (t, J = 7.2 Hz, $2 \mathrm{H}), 1.67-1.58(\mathrm{~m}, 4 \mathrm{H}), 1.53-1.45(\mathrm{~m}, 1 \mathrm{H}), 1.33-1.23(\mathrm{~m}, 4 \mathrm{H})$.

${ }^{13} \mathrm{C}$ NMR (151 MHz, $\mathrm{CDCl}_{3}$ ): $\delta 142.8,128.5,128.4,125.8,68.3,36.7,36.3$, 35.1, 33.3, 28.5.

Spectral data are in accordance with those reported in the literature. ${ }^{14}$ 


\section{4-(3-(Benzyloxy)propyl)-1-tosylpiperidine (26).}<smiles>CC(C)COCCCC1CC[Nb]C1</smiles>

From isolated redox-active ester. Following General Procedure B, with SI-25 (56.6 mg, $0.1 \mathrm{mmol}), \mathrm{Fe}(\mathrm{acac})_{3}(7.1 \mathrm{mg}, 0.020 \mathrm{mmol})$, dppBz (10.7 mg, 0.024 $\mathrm{mmol}),\left(\mathrm{BnOCH}_{2} \mathrm{CH}_{2} \mathrm{CH}_{2}\right)_{2} \mathrm{Zn}(0.15 \mathrm{mmol})$ at $0{ }^{\circ} \mathrm{C}$. Product 26 was isolated by PTLC (hexanes:ethyl acetate 10:1) to afford $21.6 \mathrm{mg}(56 \%)$ as a yellowish solid.

$\mathbf{R}_{\boldsymbol{f}}=0.30$ (hexanes:ethyl acetate 10:1).

${ }^{1} \mathrm{H}$ NMR (600 MHz, $\left.\mathrm{CDCl}_{3}\right): \delta 7.64-7.62(\mathrm{~m}, 2 \mathrm{H}), 7.34-7.27(\mathrm{~m}, 7 \mathrm{H}), 4.48(\mathrm{~s}$, 2H), $3.75(\mathrm{~d}, J=12.0 \mathrm{~Hz}, 2 \mathrm{H}), 3.42$ (t, J=6.6 Hz, 2H), 2.43 (s, 3H), 2.19 (td, J $=12.0,3.0 \mathrm{~Hz}, 2 \mathrm{H}), 1.71(\mathrm{dd}, J=13.2,2.4 \mathrm{~Hz}, 2 \mathrm{H}), 1.59-1.54(\mathrm{~m}, 2 \mathrm{H}), 1.31$

$-1.24(\mathrm{~m}, 4 \mathrm{H}), 1.17-1.10(\mathrm{~m}, 1 \mathrm{H})$.

${ }^{13} \mathrm{C}$ NMR (151 MHz, $\left.\mathrm{CDCl}_{3}\right): \delta$ 143.5, 138.6, 133.3, 129.7, 128.5, 127.9, $127.8,127.7,73.1,70.5,46.6,35.1,32.7,31.6,27.0,21.6$.

Spectral data are in accordance with those reported in the literature. 2

\section{4-cyclopropyl-1-tosylpiperidine (27).}

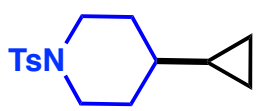

27

From isolated redox-active ester. Following General Procedure B, with SI-25 (56.6 mg, $0.1 \mathrm{mmol}), \mathrm{Fe}(\mathrm{acac})_{3}(7.1 \mathrm{mg}, 0.020 \mathrm{mmol})$, dppBz (10.7 mg, 0.024 $\mathrm{mmol})$, dicyclopropylzinc $(0.15 \mathrm{mmol})$ at r.t. Product 27 was isolated by PTLC (hexanes:ethyl acetate $10: 1)$ to afford $12.6 \mathrm{mg}(45 \%)$ as a pale white solid. $\mathbf{R}_{\boldsymbol{f}}=0.30$ (hexanes:ethyl acetate 10:1).

${ }^{1} \mathrm{H}$ NMR (600 MHz, $\left.\mathrm{CDCl}_{3}\right): \delta 7.63(\mathrm{~d}, J=8.4 \mathrm{~Hz}, 2 \mathrm{H}), 7.30(\mathrm{~d}, J=8.4 \mathrm{~Hz}$, 2H), 3.73 (d, J=11.4 Hz, 2H), $2.42(\mathrm{~s}, 3 \mathrm{H}), 2.19$ (td, $J=12.0,2.4 \mathrm{~Hz}, 2 \mathrm{H})$, $1.77(\mathrm{dd}, J=13.2,1.8 \mathrm{~Hz}, 2 \mathrm{H}), 1.46(\mathrm{qd}, J=12.0,4.2 \mathrm{~Hz}, 2 \mathrm{H}), 0.52-0.47(\mathrm{~m}$, $1 \mathrm{H}), 0.39-0.36(\mathrm{~m}, 2 \mathrm{H}), 0.00-(-0.03)(\mathrm{m}, 2 \mathrm{H})$. 
${ }^{13} \mathrm{C}$ NMR (151 MHz, $\left.\mathrm{CDCl}_{3}\right): \delta 143.4,133.4,129.7,127.9,46.6,40.7,31.3$, 21.7, 16.4, 3.3.

Spectral data are in accordance with those reported in the literature.2

\section{4-Methyl-1-tosylpiperidine (28).}<smiles>CC1CCN(C)CC1</smiles>

From isolated redox-active ester. Following General Procedure B, with SI-25 (56.6 mg, $0.1 \mathrm{mmol}), \mathrm{Fe}(\mathrm{acac})_{3}(7.1 \mathrm{mg}, 0.020 \mathrm{mmol}), \mathrm{dppBz}$ (10.7 mg, 0.024 $\mathrm{mmol}), \mathrm{Me}_{2} \mathrm{Zn}(0.15 \mathrm{mmol})$ at r.t. Product 28 was isolated by PTLC (hexanes:ethyl acetate $10: 1)$ to afford $9.4 \mathrm{mg}(37 \%)$ as a pale white solid. $\mathbf{R}_{f}=0.30$ (hexanes:ethyl acetate 10:1).

${ }^{1} \mathrm{H}$ NMR (600 MHz, $\left.\mathrm{CDCl}_{3}\right): \delta 7.64-7.62(\mathrm{~m}, 2 \mathrm{H}), 7.31(\mathrm{dd}, J=8.4,1.2 \mathrm{~Hz}$, 2H), $3.74-3.71(\mathrm{~m}, 2 \mathrm{H}), 2.43(\mathrm{~s}, 3 \mathrm{H}), 2.22(\mathrm{td}, J=11.4,2.4 \mathrm{~Hz}, 2 \mathrm{H}), 1.66-$ $1.64(\mathrm{~m}, 2 \mathrm{H}), 1.32-1.24(\mathrm{~m}, 3 \mathrm{H}), 0.90(\mathrm{~d}, J=6.0 \mathrm{~Hz}, 3 \mathrm{H})$.

${ }^{13} \mathrm{C}$ NMR (151 MHz, $\left.\mathrm{CDCl}_{3}\right): \delta 143.4,133.5,129.7,127.9,46.6,33.5,30.3$, 21.64, 21.59.

Spectral data are in accordance with those reported in the literature.2

\section{1-(Benzyloxy)-2,4-dichlorobenzene (29).}<smiles>Clc1ccc(OCc2ccccc2)c(Cl)c1</smiles>

From isolated redox-active ester. Following General Procedure B, with SI-14 (50.4 mg, $0.1 \mathrm{mmol}), \mathrm{Fe}(\mathrm{acac})_{3}(7.1 \mathrm{mg}, 0.020 \mathrm{mmol}), \mathrm{dppBz}$ (10.7 mg, 0.024 $\mathrm{mmol}), \mathrm{Ph}_{2} \mathrm{Zn}(0.15 \mathrm{mmol})$ at $0{ }^{\circ} \mathrm{C}$. Product 29 was isolated by PTLC (hexanes) to afford $19.0 \mathrm{mg}(75 \%)$ as a yellowish oil.

From in situ generation of redox-active ester: Following General Procedure C, with 2-(2,4-dichlorophenoxy)acetic acid (22.1 mg, $0.1 \mathrm{mmol})$, HATU (38.0 mg, $0.1 \mathrm{mmol}), \mathrm{Et}_{3} \mathrm{~N}$ (10.1 mg, $\left.0.1 \mathrm{mmol}\right), \mathrm{Fe}(\mathrm{acac})_{3}(7.1 \mathrm{mg}, 0.020 \mathrm{mmol}), \mathrm{dppBz}$ 
(10.7 mg, $0.024 \mathrm{mmol}), \mathrm{Ph}_{2} \mathrm{Zn}(0.25 \mathrm{mmol})$ at r.t. Product 29 was isolated by PTLC (hexanes) to afford $11.4 \mathrm{mg}(45 \%)$ as yellowish oil

$\mathbf{R}_{\boldsymbol{f}}=0.80$ (hexanes).

${ }^{1} \mathrm{H}$ NMR (600 MHz, $\left.\mathrm{CDCl}_{3}\right): \delta 7.44(\mathrm{~d}, J=9.6 \mathrm{~Hz}, 2 \mathrm{H}), 7.41-7.38(\mathrm{~m}, 3 \mathrm{H})$, 7.33 (t, $J=9.0,1.2 \mathrm{~Hz}, 1 \mathrm{H}$ ), 7.15 (dd, $J=10.8,3.6 \mathrm{~Hz}, 1 \mathrm{H}$ ), 6.88 (d, J=10.8 $\mathrm{Hz}, 1 \mathrm{H}), 5.14$ (s, 2H).

${ }^{13} \mathrm{C}$ NMR (151 MHz, $\left.\mathrm{CDCl}_{3}\right): \delta$ 153.2, 136.2, 130.2, 128.8, 128.3, 127.7, 127.2, 126.2, 124.3, 115.0, 71.3.

Spectral data are in accordance with those reported in the literature. ${ }^{15}$

(8R,9S,10S,13R,14S,17R)-10,13-dimethyl-17-(4-phenylbutan-2yl)dodecahydro-3H-cyclopenta[a]phenanthrene-3,7,12(2H,4H)-trione (30).

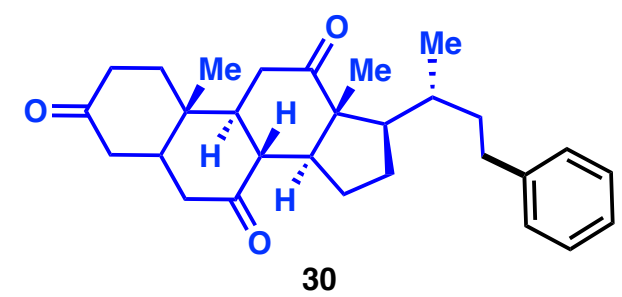

From in situ generation of redox-active ester: Following the General Procedure C with dehydrocholic acid (40 mg, $0.1 \mathrm{mmol}$ ), HATU (38 mg), $\mathrm{Et}_{3} \mathrm{~N}$ (14 $\mu \mathrm{L}), \mathrm{Fe}(\mathrm{acac})_{3}(3.5 \mathrm{mg}, 0.01 \mathrm{mmol}), \mathrm{dppBz}$ (8.9 mg, $\left.0.02 \mathrm{mmol}\right), \mathrm{Ph}_{2} \mathrm{Zn}$ $(0.25 \mathrm{mmol})$ at $25^{\circ} \mathrm{C}$. Product 30 was isolated by PTLC (hexanes:ethyl acetate $3: 2)$ to afford $27 \mathrm{mg}(62 \%)$ as a white solid.

$0.5 \mathrm{Mmol}$ from in situ generation of redox-active ester: Following the General Procedure C with dehydrocholic acid (201 mg, $0.5 \mathrm{mmol})$, HATU (190 mg), $\mathrm{Et}_{3} \mathrm{~N}(70 \mu \mathrm{L}), \mathrm{Fe}(\mathrm{acac})_{3}$ (18 mg, $\left.0.05 \mathrm{mmol}\right), \mathrm{dppBz}$ (44 mg, $\left.0.1 \mathrm{mmol}\right), \mathrm{Ph}_{2} \mathrm{Zn}$ $(1.25 \mathrm{mmol})$ at $25^{\circ} \mathrm{C}$. Product 30 was isolated by column chromatography (hexanes:ethyl acetate 1:1) to afford $132 \mathrm{mg} \mathrm{(61 \% )} \mathrm{as} \mathrm{a} \mathrm{white} \mathrm{solid.}$

Using Ni-catalysis and in situ generation of redox-active ester. Following General Procedure F, with dehydrocholic acid (40 mg, $0.1 \mathrm{mmol}$ ), HATU (38 $\mathrm{mg}$ ), $\mathrm{Et}_{3} \mathrm{~N}(14 \mu \mathrm{L}), \mathrm{NiCl}_{2} \cdot$ glyme (4.4 mg, $\left.0.02 \mathrm{mmol}\right)$, di-tert-butylbipyridine (11 
$\mathrm{mg}, 0.04 \mathrm{mmol}), \mathrm{PhZnCl}(0.3 \mathrm{mmol})$ at $25{ }^{\circ} \mathrm{C}$. Product 30 was isolated by PTLC (hexanes:ethyl acetate 3:2) to afford $22.0 \mathrm{mg}(51 \%)$ as a white solid.

m.p. $271-273^{\circ} \mathrm{C}$ (decomposition)

$\mathbf{R}_{\boldsymbol{f}}=0.33$ (hexanes:ethyl acetate 1.25:1)

${ }^{1} \mathrm{H}$ NMR (600 MHz, $\left.\mathrm{CDCl}_{3}\right)$ ठ $7.32-7.29(\mathrm{~m}, 2 \mathrm{H}), 7.22-7.16(\mathrm{~m}, 3 \mathrm{H}), 3.00-$ $2.83(\mathrm{~m}, 3 \mathrm{H}$ ), 2.77 (ddd, $J=13.5,11.0,4.9 \mathrm{~Hz}, 1 \mathrm{H}$ ), 2.51 (ddd, $J=13.6,10.7$, $6.2 \mathrm{~Hz}, 1 \mathrm{H}), 2.40-2.21(\mathrm{~m}, 5 \mathrm{H}), 2.19-2.14(\mathrm{~m}, 2 \mathrm{H}), 2.13-2.11(\mathrm{~m}, 1 \mathrm{H})$, $2.08-1.95(\mathrm{~m}, 3 \mathrm{H}), 1.88(\mathrm{td}, J=11.3,7.0 \mathrm{~Hz}, 1 \mathrm{H}), 1.78$ (dddd, $J=13.6,11.0$, 6.2, $2.7 \mathrm{~Hz}, 1 \mathrm{H}), 1.64(\mathrm{td}, J=14.4,4.6 \mathrm{~Hz}, 1 \mathrm{H}), 1.52-1.44(\mathrm{~m}, 1 \mathrm{H}), 1.43(\mathrm{~s}$, $3 \mathrm{H}), 1.39-1.24(\mathrm{~m}, 3 \mathrm{H}), 1.09(\mathrm{~s}, 3 \mathrm{H}), 0.97(\mathrm{~d}, J=6.7 \mathrm{~Hz}, 3 \mathrm{H})$.

${ }^{13} \mathrm{C}$ NMR (151 MHz, $\left.\mathrm{CDCl}_{3}\right) \delta 211.6,208.6,208.3,142.7,127.9,127.8$, 125.1, 56.5, 51.3, 48.6, 46.4, 45.3, 45.1, 44.5, 42.4, 38.2, 37.1, 36.0, 35.6, 35.4, 34.9, 32.5, 27.3, 24.7, 21.5, 18.5, 11.4.

HRMS (ESI-TOF): calc'd for $\mathrm{C}_{29} \mathrm{H}_{39} \mathrm{O}_{3}[\mathrm{M}+\mathrm{H}]^{+} 435.2894$; found 435.2899.

\section{2-(3-Methyl-1-phenylbutyl)isoindoline-1,3-dione (31).}<smiles>CCCC(c1ccccc1)N1C(=O)c2ccccc2C1=O</smiles>

From isolated redox-active ester. Following General Procedure B, with SI-26 (40.6 mg, $0.1 \mathrm{mmol}), \mathrm{Fe}(\mathrm{acac})_{3}(7.1 \mathrm{mg}, 0.020 \mathrm{mmol}), \mathrm{dppBz}$ (10.7 mg, 0.024 $\mathrm{mmol}), \mathrm{Ph}_{2} \mathrm{Zn}(0.15 \mathrm{mmol})$ at $0{ }^{\circ} \mathrm{C}$. Product 31 was isolated by PTLC (hexanes:ethyl acetate $10: 1)$ to afford $12.1 \mathrm{mg}(41 \%)$ as a pale white solid in racemic form.

From in situ generation of redox-active ester following General Procedure C: Activation was carried out with (R)-2-(1,3-dioxoisoindolin-2-yl)-4methylpentanoic acid $(26.1 \mathrm{mg}, 0.1 \mathrm{mmol})$, HATU (38.0 $\mathrm{mg}, 0.1 \mathrm{mmo})$, and $\mathrm{Et}_{3} \mathrm{~N}(10.1 \mathrm{mg}, 0.1 \mathrm{mmol})$ in $0.2 \mathrm{~mL}$ of $\mathrm{THF}$, followed by addition of $\mathrm{Fe}(\mathrm{acac})_{3}$ 
(7.1 mg, $0.020 \mathrm{mmol}$ ) and dppBz (10.7 mg, $0.024 \mathrm{mmol}$ ) in $0.5 \mathrm{~mL}$ of toluene. $\mathrm{Ph}_{2} \mathrm{Zn}(0.15 \mathrm{mmol})$ was added at r.t. Product $\mathbf{3 1}$ was isolated by PTLC (hexanes:ethyl acetate $10: 1)$ to afford $19.3 \mathrm{mg}(66 \%)$ as a pale white solid in racemic form.

$1.0 \mathrm{Mmol}$ scale from in situ generation of redox-active ester following General Procedure C: Activation was carried out with $(R)-2-(1,3-d i o x o i s o i n d o l i n-2-y \mid)-4-$ methylpentanoic acid (261 mg, $1.0 \mathrm{mmol}$ ), HATU (380 mg, $1.0 \mathrm{mmol}$ ), and $\mathrm{Et}_{3} \mathrm{~N}(101 \mathrm{mg}, 1.0 \mathrm{mmol})$ in $2.0 \mathrm{~mL}$ of $\mathrm{THF}$, followed by addition of $\mathrm{Fe}(\mathrm{acac})_{3}$ $(71 \mathrm{mg}, 0.20 \mathrm{mmol})$ and dppBz (107 mg, $0.24 \mathrm{mmol})$ in $5.0 \mathrm{~mL}$ of toluene. $\mathrm{Ph}_{2} \mathrm{Zn}(4.6 \mathrm{~mL}, 0.33 \mathrm{M}$ in THF, $1.5 \mathrm{mmol}$ ) was added at r.t. Product 31 was isolated by PTLC (hexanes:ethyl acetate $10: 1$ ) to afford $220.9 \mathrm{mg}(75 \%)$ as a pale white solid in racemic form.

m.p. $82-83^{\circ} \mathrm{C}$

$\mathbf{R}_{\boldsymbol{f}}=0.30$ (hexanes:ethyl acetate 10:1).

${ }^{1} \mathrm{H}$ NMR (500 MHz, $\mathbf{C D C l}_{3}$ ): $\delta 7.81-7.78(\mathrm{~m}, 2 \mathrm{H}), 7.70-7.67(\mathrm{~m}, 2 \mathrm{H}), 7.55$ (d, $J=7.5 \mathrm{~Hz}, 2 \mathrm{H}$ ), 7.32 (t, $J=7.5 \mathrm{~Hz}, 2 \mathrm{H}$ ), 7.25 (t, $J=7.5 \mathrm{~Hz}, 1 \mathrm{H}$ ), 5.45 (dd, $J=10.0,6.5 \mathrm{~Hz}, 1 \mathrm{H}), 2.64-2.58(\mathrm{~m}, 1 \mathrm{H}), 2.64-2.58(\mathrm{~m}, 1 \mathrm{H}), 2.06-2.00$ $(\mathrm{m}, 1 \mathrm{H}), 1.57-1.49(\mathrm{~m}, 1 \mathrm{H}), 0.98(\mathrm{~d}, J=4.0 \mathrm{~Hz}, 3 \mathrm{H}), 0.97(\mathrm{~d}, J=4.0 \mathrm{~Hz}, 3 \mathrm{H})$. ${ }^{13} \mathrm{C}$ NMR (151 MHz, $\left.\mathrm{CDCl}_{3}\right): \delta 168.6,140.1,134.0,132.0,128.6,128.3$, $127.8,123.3,53.2,39.9,25.6,23.1,22.1$.

HRMS (ESI-TOF): calc'd for $\mathrm{C}_{19} \mathrm{H}_{19} \mathrm{NO}_{2}[\mathrm{M}+\mathrm{H}]^{+} 294.1488$; found 294.1485 .

\section{2-(1,2-diphenylethyl)isoindoline-1,3-dione (32).}<smiles>O=C1c2ccccc2C(=O)N1C(Cc1ccccc1)c1ccccc1</smiles>

32

From in situ generation of redox-active ester following General Procedure C: Activation was carried out with (R)-2-(1,3-dioxoisoindolin-2-yl)-3phenylpropanoic acid (29.5 mg, $0.1 \mathrm{mmol})$, HATU (38.0 mg, $0.1 \mathrm{mmol})$ and $\mathrm{Et}_{3} \mathrm{~N}(10.1 \mathrm{mg}, 0.1 \mathrm{mmol})$ in $0.2 \mathrm{~mL}$ of $\mathrm{THF}$, followed by addition of $\mathrm{Fe}(\mathrm{acac})_{3}$ 
(7.1 mg, $0.020 \mathrm{mmol}$ ) and dppBz (10.7 mg, $0.024 \mathrm{mmol}$ ) in $0.5 \mathrm{~mL}$ of toluene. $\mathrm{Ph}_{2} \mathrm{Zn}(0.15 \mathrm{mmol})$ was added at r.t. Product 32 was isolated by PTLC (hexanes:ethyl acetate $10: 1$ ) to afford $15.0 \mathrm{mg} \mathrm{(46 \% )}$ ) as a pale white solid in a racemic form. 
$\mathbf{R}_{\boldsymbol{f}}=0.30$ (hexanes:ethyl acetate 10:1).

${ }^{1} \mathrm{H}$ NMR $\left(500 \mathrm{MHz}, \mathrm{CDCl}_{3}\right): \delta 7.73(\mathrm{dd}, J=5.0,3.0 \mathrm{~Hz}, 2 \mathrm{H}), 7.64-7.60(\mathrm{~m}$, 4H), 7.35 (t, $J=7.5 \mathrm{~Hz}, 2 \mathrm{H}), 7.28$ (t, $J=7.0 \mathrm{~Hz}, 1 \mathrm{H}), 7.24-7.18(\mathrm{~m}, 4 \mathrm{H}), 7.12$ $(\mathrm{t}, J=7.0 \mathrm{~Hz}, 1 \mathrm{H}), 5.67(\mathrm{dd}, J=11.0,6.0 \mathrm{~Hz}, 1 \mathrm{H}), 3.99(\mathrm{dd}, J=14.0,11.5 \mathrm{~Hz}$, $1 \mathrm{H}), 3.51(\mathrm{dd}, J=14.5,6.0 \mathrm{~Hz}, 1 \mathrm{H})$.

${ }^{13} \mathrm{C}$ NMR (151 MHz, $\left.\mathrm{CDCl}_{3}\right): \delta$ 168.4, 139.5, 138.1, 134.0, 131.8, 129.0, 128.8, 128.6, 128.2, 128.1, 126.7, 123.3, 56.2, 37.2.

Spectral data are in accordance with those reported in the literature. ${ }^{16}$

((8Z,11Z)-Heptadeca-8,11-dien-1-yl)benzene (33).<smiles>C1=CCCCCC1</smiles>

33

From isolated redox-active ester. Following General Procedure B, with SI-15 (56.3 mg, $0.1 \mathrm{mmol}), \mathrm{Fe}(\mathrm{acac})_{3}(7.1 \mathrm{mg}, 0.020 \mathrm{mmol})$, dppBz (10.7 mg, 0.024 $\mathrm{mmol}), \mathrm{Ph}_{2} \mathrm{Zn}(0.15 \mathrm{mmol})$ at $0{ }^{\circ} \mathrm{C}$. Product 33 was isolated by PTLC (hexanes) to afford $21.3 \mathrm{mg}(68 \%)$ as yellowish oil.

From in situ generation of redox-active ester: Following the General Procedure C with linoleic acid (28 mg, $0.1 \mathrm{mmol}$ ), HATU (38 mg), $\mathrm{Et}_{3} \mathrm{~N}$ (14 $\mu \mathrm{L}), \mathrm{Fe}(\mathrm{acac})_{3}$ (3.5 mg, $\left.0.01 \mathrm{mmol}\right), \mathrm{dppBz}(8.9 \mathrm{mg}, 0.02 \mathrm{mmol}), \mathrm{Ph}_{2} \mathrm{Zn}(0.25$ $\mathrm{mmol}$ ) at $25{ }^{\circ} \mathrm{C}$. Product 33 was isolated by PTLC (hexanes) to afford $25 \mathrm{mg}$ $(80 \%)$ as yellow oil.

$0.5 \mathrm{Mmol}$ scale from in situ generation of redox-active ester: Following the General Procedure C with linoleic acid (140 mg, $0.05 \mathrm{mmol})$, HATU (190 mg), $\mathrm{Et}_{3} \mathrm{~N}$ (70 $\mu \mathrm{L}$ ), $\mathrm{Fe}(\mathrm{acac})_{3}$ (18 mg, $0.1 \mathrm{mmol}$ ), dppBz (44 mg, $0.2 \mathrm{mmol}$ ), $\mathrm{Ph}_{2} \mathrm{Zn}$ $(1.25 \mathrm{mmol})$ at $25^{\circ} \mathrm{C}$. Product 33 was isolated by column chromatography (hexanes) to afford $121 \mathrm{mg}(76 \%)$ as yellow oil. 
Using Ni-catalysis and in situ generation of redox-active ester. Following General Procedure F, with linoleic acid (28 mg, $0.1 \mathrm{mmol})$, HATU (38 mg), $\mathrm{Et}_{3} \mathrm{~N}(14 \mu \mathrm{L}), \mathrm{NiCl}_{2} \cdot g l y m e(4.4 \mathrm{mg}, 0.02 \mathrm{mmol})$, di-tert-butylbipyridine (11 mg, $0.04 \mathrm{mmol}), \mathrm{PhZnCl}(0.3 \mathrm{mmol})$ at $25^{\circ} \mathrm{C}$. Product 33 was isolated by PTLC (hexanes) to afford $7.0 \mathrm{mg}(23 \%)$ as a white solid.

$\mathbf{R}_{f}=0.90$ (hexanes)

${ }^{1} \mathrm{H}$ NMR (600 MHz, $\left.\mathrm{CDCl}_{3}\right): \delta 7.29-7.26(\mathrm{~m}, 2 \mathrm{H}), 7.19-7.16(\mathrm{~m}, 3 \mathrm{H}), 5.42-$ $5.32(\mathrm{~m}, 4 \mathrm{H}), 2.78(\mathrm{t}, J=7.2 \mathrm{~Hz}, 2 \mathrm{H}), 2.61(\mathrm{t}, J=7.8 \mathrm{~Hz}, 2 \mathrm{H}), 2.08-2.02(\mathrm{~m}$, $4 \mathrm{H}), 2.08-2.02(\mathrm{~m}, 4 \mathrm{H}), 1.65-1.60(\mathrm{~m}, 2 \mathrm{H}), 1.39-1.27(\mathrm{~m}, 16 \mathrm{H}), 0.90(\mathrm{t}, J$ $=6.6 \mathrm{~Hz}, 3 \mathrm{H})$.

${ }^{13} \mathrm{C}$ NMR (151 MHz, $\mathrm{CDCl}_{3}$ ): $\delta$ 143.1, 130.4, 130.3, 128.5, 128.4, 128.2, 128.1, 125.7, 36.1, 31.7, 31.7, 29.8, 29.6, 29.5, 29.5, 29.4, 27.4, 25.8, 22.7, 14.2.

MS (GCMS-CI): m/z 312, 207, 131, 117, 104, 91, 81, 67, 55.

\section{2-Phenyladamantane (34).}

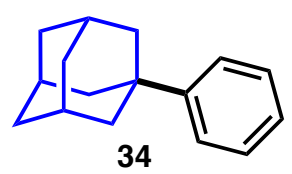

From isolated redox-active ester. Following General Procedure B, with SI-16 (33 mg, $0.1 \mathrm{mmol}), \mathrm{Fe}(\mathrm{acac})_{3}(14.1 \mathrm{mg}, 0.04 \mathrm{mmol})$, dppBz (21.4 mg, 0.048 $\mathrm{mmol})$, toluene $(0.5 \mathrm{~mL})$, and $\mathrm{Ph}_{2} \mathrm{Zn}(0.25 \mathrm{mmol})$ at $25^{\circ} \mathrm{C}$. Product 34 was isolated by PTLC (hexanes) to afford $13.1 \mathrm{mg}(62 \%)$ as a white solid.

1.0 Mmol from isolated redox-active ester: Following General Procedure B, with SI-16 (330 mg, $1.0 \mathrm{mmol}), \mathrm{Fe}(\mathrm{acac})_{3}(141 \mathrm{mg}, 0.4 \mathrm{mmol}), \mathrm{dppBz}(214$ $\mathrm{mg}, 0.48 \mathrm{mmol})$, toluene $(5 \mathrm{~mL})$, and $\mathrm{Ph}_{2} \mathrm{Zn}(2.5 \mathrm{mmol})$ at $25^{\circ} \mathrm{C}$. Product 34 was isolated by PTLC (hexanes) to afford $125 \mathrm{mg} \mathrm{(59 \% )}$ as a white solid.

From redox-active ester generated in situ with HATU: Following General Procedure C, with adamantane-1-carboxylic acid (18 $\mathrm{mg}, 0.1 \mathrm{mmol}$ ), HATU 
(38 mg, $0.1 \mathrm{mmol}), \mathrm{Et}_{3} \mathrm{~N}(14 \mu \mathrm{L}, 0.1 \mathrm{mmol}), \mathrm{Fe}(\mathrm{acac})_{3}(7.1 \mathrm{mg}, 0.02 \mathrm{mmol})$, dppBz (17.9 mg, $0.04 \mathrm{mmol}), \mathrm{Ph}_{2} \mathrm{Zn}(0.25 \mathrm{mmol})$ at $25^{\circ} \mathrm{C}$. Product 34 was isolated by PTLC (hexanes) to afford $3.2 \mathrm{mg}(15 \%)$ as a white solid.

From redox-active ester generated in situ with HBTU: Following General Procedure $\mathrm{C}$, with adamantane-1-carboxylic acid (18 $\mathrm{mg}, 0.1 \mathrm{mmol}$ ), HBTU (38 mg, $0.1 \mathrm{mmol}), \mathrm{Et}_{3} \mathrm{~N}$ (14 $\left.\mu \mathrm{L}, 0.1 \mathrm{mmol}\right), \mathrm{Fe}(\mathrm{acac})_{3}(7.1 \mathrm{mg}, 0.02 \mathrm{mmol})$, dppBz (17.9 mg, $0.04 \mathrm{mmol}), \mathrm{Ph}_{2} \mathrm{Zn}(0.25 \mathrm{mmol})$ at $25^{\circ} \mathrm{C}$. Product 34 was isolated by PTLC (hexanes) to afford $11.6 \mathrm{mg}(55 \%)$ as a white solid.

Kumada-coupling using Fe-catalysis from isolated redox-active ester. Following General Procedure D, with SI-16 (33 mg, $0.1 \mathrm{mmol}$ ), Fe(acac) 3 (35 $\mathrm{mg}, 0.1 \mathrm{mmol}), \mathrm{PhMgBr} \cdot \mathrm{LiCl}(0.3 \mathrm{mmol})$ at $25^{\circ} \mathrm{C}$. Product 34 was isolated by PTLC (hexanes) to afford $12.4 \mathrm{mg}(58 \%)$ as a white solid.

Negishi-coupling using Ni-catalysis. Following General Procedure E, with SI16 (33 mg, $0.1 \mathrm{mmol}), \mathrm{Ni}(\mathrm{acac})_{2}(5.1 \mathrm{mg}, 0.02 \mathrm{mmol}), 6,6$ '-dimethyl-2,2'bipyridine ( $3.7 \mathrm{mg}, 0.02 \mathrm{mmol}), \mathrm{PhZnCl}(0.2 \mathrm{mmol}, c=0.9 \mathrm{M}$ in THF) in acetonitrile $(0.2 \mathrm{~mL})$ at $80{ }^{\circ} \mathrm{C}$ overnight. Product 34 was isolated by PTLC (hexanes) to afford $8.1 \mathrm{mg}(38 \%)$ as a white solid.

$\mathbf{R}_{f}=0.47$ (hexanes)

${ }^{1} \mathrm{H}$ NMR $\left(600 \mathrm{MHz}, \mathrm{CDCl}_{3}\right) \delta 7.38(\mathrm{~d}, J=7.5 \mathrm{~Hz}, 2 \mathrm{H}), 7.33(\mathrm{t}, J=7.8 \mathrm{~Hz}, 2 \mathrm{H})$, $7.18(\mathrm{t}, J=7.2 \mathrm{~Hz}, 1 \mathrm{H}), 2.11(\mathrm{~m}, 3 \mathrm{H}), 1.93(\mathrm{~d}, J=3.0 \mathrm{~Hz}, 6 \mathrm{H}), 1.84-1.74(\mathrm{~m}$, $6 \mathrm{H})$.

${ }^{13} \mathrm{C}$ NMR (151 MHz, $\left.\mathrm{CDCl}_{3}\right) \delta 151.5,128.2,125.6,125.0,43.3,37.0,36.3$, 29.1.

Spectral data are in accordance with those reported in the literature. ${ }^{17}$ 
Methyl 4-phenylbicyclo[2.2.2]octane-1-carboxylate (35).

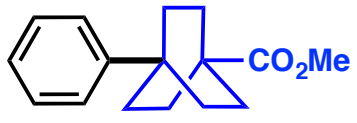

35

From isolated redox-active ester. Following General Procedure B, with SI-17 (36 mg, $0.1 \mathrm{mmol}$ ), $\mathrm{Fe}(\mathrm{acac})_{3}(14.1 \mathrm{mg}, 0.04 \mathrm{mmol}$ ), dppBz (21.4 mg, 0.048 $\mathrm{mmol})$, toluene $(0.5 \mathrm{~mL})$, and $\mathrm{Ph}_{2} \mathrm{Zn}(0.25 \mathrm{mmol})$ at $25^{\circ} \mathrm{C}$. Product 35 was isolated by PTLC (hexanes:ethyl acetate 10:1 and toluene) to afford $13.7 \mathrm{mg}$ $(56 \%)$ as a white solid.

Negishi-coupling using Ni-catalysis. Following General Procedure E, with SI17 (36 mg, $0.1 \mathrm{mmol}), \mathrm{Ni}(\mathrm{acac})_{2}(5.1 \mathrm{mg}, 0.02 \mathrm{mmol}), 6,6$ '-dimethyl-2,2'bipyridine $(3.7 \mathrm{mg}, 0.02 \mathrm{mmol}), \mathrm{PhZnCl}(0.2 \mathrm{mmol}, c=0.9 \mathrm{M}$ in THF) in acetonitrile $(0.2 \mathrm{~mL})$ at $80^{\circ} \mathrm{C}$ overnight. Product 35 was isolated by PTLC (hexanes:ethyl acetate $10: 1)$ to afford $10.6 \mathrm{mg}(43 \%)$ as a white solid.

$\mathbf{R}_{\boldsymbol{f}}=0.40$ (hexanes:ethyl acetate 10:1)

${ }^{1} \mathrm{H}$ NMR (600 MHz, $\left.\mathrm{CDCl}_{3}\right) \delta 7.34-7.28(\mathrm{~m}, 4 \mathrm{H}), 7.20-7.16(\mathrm{~m}, 1 \mathrm{H}), 3.68$ (s, 3H), $1.95-1.90(\mathrm{~m}, 6 \mathrm{H}), 1.89-1.85(\mathrm{~m}, 6 \mathrm{H})$.

${ }^{13} \mathrm{C}$ NMR (151 MHz, $\left.\mathrm{CDCl}_{3}\right) \delta 178.6,149.3,128.3,125.9,125.6,51.8,39.2$, 34.7, 31.8, 28.9.

Spectral data are in accordance with those reported in the literature. ${ }^{18}$

\section{1,4-Diphenyl-2-oxabicyclo[2.2.2]octane (36).}

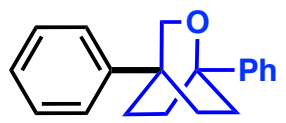

36

From isolated redox-active ester. Following General Procedure B, with SI-29 (38 mg, $0.1 \mathrm{mmol}), \mathrm{Fe}(\mathrm{acac})_{3}$ (14.1 mg, $\left.0.04 \mathrm{mmol}\right), \mathrm{dppBz}$ (21.4 mg, 0.048 mmol), toluene $(0.5 \mathrm{~mL})$, and $\mathrm{Ph}_{2} \mathrm{Zn}(0.25 \mathrm{mmol})$ at $25{ }^{\circ} \mathrm{C}$. Product 36 was 
isolated by PTLC (hexanes:ethyl acetate $4: 1$ and $\mathrm{CH}_{2} \mathrm{Cl}_{2}$ ) to afford $7.9 \mathrm{mg}$ $(30 \%)$ as a white solid.

From redox-active ester generated in situ with HATU: Following General Procedure $\mathrm{C}$, with 1-phenyl-2-oxabicyclo[2.2.2]octane-4-carboxylic acid (23 $\mathrm{mg}, 0.1 \mathrm{mmol}$ ), HATU (38 mg, $0.1 \mathrm{mmol}), \mathrm{Et}_{3} \mathrm{~N}(14 \mu \mathrm{L}, 0.1 \mathrm{mmol}), \mathrm{Fe}(\mathrm{acac})_{3}$ (7.1 mg, $0.02 \mathrm{mmol}), \mathrm{dppBz}$ (17.9 mg, $0.04 \mathrm{mmol}), \mathrm{Ph}_{2} \mathrm{Zn}(0.25 \mathrm{mmol})$ at 25 ${ }^{\circ} \mathrm{C}$. Product 36 was isolated by PTLC (hexanes: ethyl acetate $4: 1$ and $\mathrm{CH}_{2} \mathrm{Cl}_{2}$ ) to afford $6.8 \mathrm{mg}(26 \%)$ as a white solid.

Negishi-coupling using Ni-catalysis. Following General Procedure E, with SI29 (19 mg, $0.05 \mathrm{mmol}), \mathrm{Ni}(\mathrm{acac})_{2}(2.6 \mathrm{mg}, 0.01 \mathrm{mmol}), 6,6$ '-dimethyl-2,2'bipyridine $(1.8 \mathrm{mg}, 0.01 \mathrm{mmol}), \mathrm{PhZnCl}(0.1 \mathrm{mmol}, c=0.9 \mathrm{M}$ in THF) in acetonitrile $(0.1 \mathrm{~mL})$ at $80{ }^{\circ} \mathrm{C}$ overnight. Product 36 was isolated by PTLC $\left(\mathrm{CH}_{2} \mathrm{Cl}_{2}\right)$ to afford $1.4 \mathrm{mg}(11 \%)$ as a white solid.

$\mathbf{R}_{\boldsymbol{f}}=0.68\left(\mathrm{CH}_{2} \mathrm{Cl}_{2}\right)$

${ }^{1} \mathrm{H}$ NMR (600 MHz, $\left.\mathrm{CDCl}_{3}\right) \delta 7.49-7.39(\mathrm{~m}, 1 \mathrm{H}), 7.40-7.30(\mathrm{~m}, 3 \mathrm{H}), 7.26-$ $7.22(\mathrm{~m}, 1 \mathrm{H}), 4.22(\mathrm{dd}, J=1.9,1.2 \mathrm{~Hz}, 1 \mathrm{H}), 2.25-2.16(\mathrm{~m}, 3 \mathrm{H}), 2.09$ (ddt, $J=$ $11.0,7.9,1.8 \mathrm{~Hz}, 1 \mathrm{H})$.

${ }^{13} \mathrm{C}$ NMR (151 MHz, $\left.\mathrm{CDCl}_{3}\right) \delta 145.9,128.0,127.6,126.2,126.0,125.1$, 124.2, 73.7, 72.0, 35.3, 33.1, 30.8.

HRMS (ESI-TOF): calc'd for $\mathrm{C}_{19} \mathrm{H}_{21} \mathrm{O}[\mathrm{M}+\mathrm{H}]^{+} 265.1587$; found 265.1588 .

Methyl 3-phenylbicyclo[1.1.1]pentane-1-carboxylate (37).

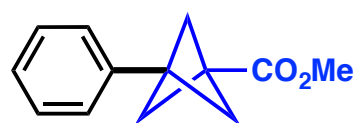

37

From isolated redox-active ester. Following General Procedure B, with SI-30 (32 mg, $0.1 \mathrm{mmol}), \mathrm{Fe}(\mathrm{acac})_{3}(14.1 \mathrm{mg}, 0.04 \mathrm{mmol}), \mathrm{dppBz}$ (21.4 mg, 0.048 $\mathrm{mmol})$, toluene $(0.5 \mathrm{~mL})$, and $\mathrm{Ph}_{2} \mathrm{Zn}(0.25 \mathrm{mmol})$ at $25^{\circ} \mathrm{C}$. Product 37 was isolated by PTLC (hexanes:ethyl acetate $1.25: 1$ ) to afford $7.1 \mathrm{mg}(35 \%)$ as a white solid. 
$\mathbf{R}_{f}=0.80$ (hexanes:ethyl acetate 1.25:1).

${ }^{1} \mathrm{H}$ NMR (600 MHz, Acetone- $d_{6}$ ) $\delta 7.33$ (ddt, $J=7.8,7.0,0.8 \mathrm{~Hz}, 2 \mathrm{H}$ ), $7.30-$ $7.24(\mathrm{~m}, 3 \mathrm{H}), 3.68(\mathrm{~s}, 3 \mathrm{H}), 2.31(\mathrm{~s}, 6 \mathrm{H})$.

${ }^{13} \mathrm{C}$ NMR (151 MHz, Acetone- $\left.d_{6}\right) \delta 169.2,139.3,127.7,126.3,125.5,53.6$, $52.4,50.4,41.0$.

Spectral data are in accordance with those reported in the literature. ${ }^{19}$ 
Methyl 4-phenylcubane-1-carboxylate (38).

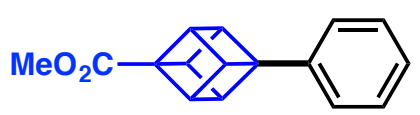

38

From isolated redox-active ester. Following General Procedure B, with SI-31 (35 mg, $0.1 \mathrm{mmol}), \mathrm{Fe}(\mathrm{acac})_{3}(14.1 \mathrm{mg}, 0.04 \mathrm{mmol}), \mathrm{dppBz}(21.4 \mathrm{mg}, 0.048$ $\mathrm{mmol})$, toluene $(0.5 \mathrm{~mL})$, and $\mathrm{Ph}_{2} \mathrm{Zn}(0.25 \mathrm{mmol})$ at $25{ }^{\circ} \mathrm{C}$. Product 38 was isolated by PTLC (hexanes: ethyl acetate $10: 1, \mathrm{CH}_{2} \mathrm{Cl}_{2}$, and toluene) to afford $6.0 \mathrm{mg}(25 \%)$ as a pale yellow solid.

From redox-active ester generated in situ with HATU, see "iterative diarylation of cubane" (Section 11)

m.p. $78-80^{\circ} \mathrm{C}$

${ }^{1} \mathrm{H}$ NMR (600 MHz, $\left.\mathrm{CDCl}_{3}\right) \delta 7.39-7.34(\mathrm{~m}, 2 \mathrm{H}), 7.23-7.18(\mathrm{~m}, 3 \mathrm{H}), 4.28-$ $4.21(\mathrm{~m}, 3 \mathrm{H}), 4.19-4.13(\mathrm{~m}, 3 \mathrm{H}), 3.74(\mathrm{~s}, 3 \mathrm{H})$.

${ }^{13} \mathrm{C}$ NMR (151 MHz, $\left.\mathrm{CDCl}_{3}\right) \delta 172.9,142.2,128.6,126.3,124.9,60.4,56.6$, $51.7,48.9,46.2$.

HRMS (ESI-TOF): calc'd for $\mathrm{C}_{16} \mathrm{H}_{15} \mathrm{O}_{2}[\mathrm{M}+\mathrm{H}]^{+}$239.1067; found 239.1068.

Cyclopropane-1,1-diyldibenzene (39).<smiles>c1ccc(C2(c3ccccc3)CC2)cc1</smiles>

39

From isolated redox-active ester. Following General Procedure B, with SI-32 (31 mg, $0.1 \mathrm{mmol}), \mathrm{Fe}(\mathrm{acac})_{3}(14.1 \mathrm{mg}, 0.04 \mathrm{mmol}), \mathrm{dppBz}(21.4 \mathrm{mg}, 0.048$ $\mathrm{mmol})$, toluene $(0.5 \mathrm{~mL})$, and $\mathrm{Ph}_{2} \mathrm{Zn}(0.25 \mathrm{mmol})$ at $25{ }^{\circ} \mathrm{C}$. Product 39 was isolated by PTLC (pentane) to afford $8.5 \mathrm{mg}(44 \%)$ as a colorless oil.

From redox-active ester generated in situ with HATU: Following General Procedure C, with 1-phenylcyclopropane-1-carboxylic acid (16 mg, $0.1 \mathrm{mmol}$ ), HATU (38 mg, $0.1 \mathrm{mmol}), \mathrm{Et}_{3} \mathrm{~N}$ (14 $\left.\mu \mathrm{L}, 0.1 \mathrm{mmol}\right), \mathrm{Fe}(\mathrm{acac})_{3}(7.1 \mathrm{mg}, 0.02$ 
mmol), dppBz (17.9 mg, $0.04 \mathrm{mmol}), \mathrm{Ph}_{2} \mathrm{Zn}(0.25 \mathrm{mmol})$ at $25^{\circ} \mathrm{C}$. Product 39 was isolated by PTLC (pentane) to afford $12.3 \mathrm{mg}(63 \%)$ as a colorless oil.

1.0 $\mathrm{Mmol}$ from redox-active ester generated in situ with HATU: Following General Procedure C, with 1-phenylcyclopropane-1-carboxylic acid (162 mg, $1.0 \mathrm{mmol}), \operatorname{HATU}(380 \mathrm{mg}, 1.0 \mathrm{mmol}), \mathrm{Et}_{3} \mathrm{~N}$ (101 mg, $\left.0.1 \mathrm{mmol}\right), \mathrm{Fe}(\mathrm{acac})_{3}$ (71 $\mathrm{mg}, 0.20 \mathrm{mmol})$ and dppBz (179 mg, $0.4 \mathrm{mmol}), \mathrm{Ph}_{2} \mathrm{Zn}(2.5 \mathrm{mmol})$ at 25 ${ }^{\circ} \mathrm{C}$. Product 39 (126.3 mg, 65\%) and biphenyl (86.7 mg) were isolated as mixture by PTLC (pentane).

Negishi-coupling using Ni-catalysis. Following General Procedure E, with SI32 (31 mg, $0.1 \mathrm{mmol}), \mathrm{Ni}(\mathrm{acac})_{2}$ (5.1 mg, $\left.0.02 \mathrm{mmol}\right)$, 6,6'-dimethyl-2,2'bipyridine (3.7 mg, $0.02 \mathrm{mmol}), \mathrm{PhZnCl}(0.2 \mathrm{mmol}, c=0.9 \mathrm{M}$ in THF) in acetonitrile $(0.2 \mathrm{~mL})$ at $80{ }^{\circ} \mathrm{C}$ overnight. Product 39 was isolated by PTLC (heptane) to afford $3.2 \mathrm{mg}(16 \%)$ as a colorless oil.

$\mathbf{R}_{f}=0.37$ (hexanes)

${ }^{1} \mathrm{H}$ NMR (600 MHz, $\left.\mathrm{CDCl}_{3}\right) \delta 7.27(\mathrm{t}, J=7.4 \mathrm{~Hz}, 4 \mathrm{H}), 7.24-7.21(\mathrm{~m}, 4 \mathrm{H})$, $7.20-7.15(\mathrm{~m}, 2 \mathrm{H}), 1.30(\mathrm{~s}, 4 \mathrm{H})$.

${ }^{13} \mathrm{C}$ NMR (151 MHz, $\mathrm{CDCl}_{3}$ ) $\delta$ 145.9, 128.6, 128.4, 126.1, 30.0, 16.6.

Spectral data are in accordance with those reported in the literature. ${ }^{20}$

\section{1-Chloro-4-(1-phenylcyclopropyl)benzene (40).}<smiles>Clc1ccc(C2(c3ccccc3)CC2)cc1</smiles>

From isolated redox-active ester. Following General Procedure B, with SI-33 (34 mg, $0.1 \mathrm{mmol}), \mathrm{Fe}(\mathrm{acac})_{3}(14.1 \mathrm{mg}, 0.04 \mathrm{mmol}), \mathrm{dppBz}(21.4 \mathrm{mg}, 0.048$ $\mathrm{mmol}), \mathrm{Ph}_{2} \mathrm{Zn}(0.25 \mathrm{mmol})$ at $25{ }^{\circ} \mathrm{C}$. Product 40 was isolated by PTLC (pentane) to afford $8.9 \mathrm{mg}(39 \%)$ as a colorless oil.

From redox-active ester generated in situ with HATU: Following General Procedure C, with 1-(4-chlorophenyl)cyclopropane-1-carboxylic acid (20 mg, 
$0.1 \mathrm{mmol}$ ), HATU (38 mg, $0.1 \mathrm{mmol}), \mathrm{Et}_{3} \mathrm{~N}$ (14 $\left.\mu \mathrm{L}, 0.1 \mathrm{mmol}\right), \mathrm{Fe}(\mathrm{acac})_{3}(7.1$ $\mathrm{mg}, 0.02 \mathrm{mmol}), \mathrm{dppBz}$ (17.9 mg, $0.04 \mathrm{mmol}), \mathrm{Ph}_{2} \mathrm{Zn}(0.25 \mathrm{mmol})$ at $25^{\circ} \mathrm{C}$. Product 40 was isolated by PTLC (pentane) to afford $14.6 \mathrm{mg}(64 \%)$ as a colorless oil.

Negishi-coupling using Ni-catalysis. Following General Procedure E, with SI33 (34 mg, $0.1 \mathrm{mmol}), \mathrm{Ni}(\mathrm{acac})_{2}(5.1 \mathrm{mg}, 0.02 \mathrm{mmol}), 6,6$ '-dimethyl-2,2'bipyridine $(3.7 \mathrm{mg}, 0.02 \mathrm{mmol}), \mathrm{PhZnCl}(0.2 \mathrm{mmol}, c=0.9 \mathrm{M}$ in THF) in acetonitrile $(0.2 \mathrm{~mL})$ at $80^{\circ} \mathrm{C}$ overnight. Product 40 was isolated by PTLC (heptane) to afford $4.4 \mathrm{mg}(19 \%)$ as a colorless oil.

$\mathbf{R}_{f}=0.43$ (hexanes)

${ }^{1} \mathrm{H}$ NMR (600 MHz, $\left.\mathrm{CDCl}_{3}\right) \delta 7.28(\mathrm{t}, J=8.5 \mathrm{~Hz}, 2 \mathrm{H}), 7.23(\mathrm{~d}, J=8.5 \mathrm{~Hz}, 2 \mathrm{H})$, $7.22-7.18(\mathrm{~m}, 3 \mathrm{H}), 7.16(\mathrm{~d}, J=8.5 \mathrm{~Hz}, 2 \mathrm{H}), 1.34-1.30(\mathrm{~m}, 2 \mathrm{H}), 1.29-1.25$ $(\mathrm{m}, 2 \mathrm{H})$.

${ }^{13} \mathrm{C}$ NMR (151 MHz, $\left.\mathrm{CDCl}_{3}\right) \delta 145.3,144.4,131.8,129.9,128.5,128.5$, 128.5, 126.3, 29.6, 16.6 .

HRMS (GCMS-CI): m/z 228 (M+), 193, 178, 165, 150

(S)-Benzyl 2-(tert-butoxycarbonylamino)-4-phenylbutanoate (42).<smiles>CC(C)(C)OC(=O)N[C@@H](CCc1ccccc1)C(=O)OCc1ccccc1</smiles>

From isolated redox-active ester. Following General Procedure B, with SI-21 (62.1 mg, $0.1 \mathrm{mmol}), \mathrm{Fe}(\mathrm{acac})_{3}(7.1 \mathrm{mg}, 0.020 \mathrm{mmol}), \mathrm{dppBz}$ (10.7 mg, 0.024 mmol), $\mathrm{Ph}_{2} \mathrm{Zn}(0.15 \mathrm{mmol})$ at r.t. Product 42 was isolated by PTLC (hexanes:ethyl acetate $6: 1)$ to afford $9.8 \mathrm{mg}(27 \%)$ as a yellowish oil.

$\mathbf{R}_{\mathbf{f}}=0.30$ (hexanes:ethyl acetate $5: 1$ ).

${ }^{1} \mathrm{H}$ NMR $\left(600 \mathrm{MHz}, \mathrm{CDCl}_{3}\right): \delta 7.38-7.32(\mathrm{~m}, 5 \mathrm{H}), 7.26(\mathrm{t}, J=7.8 \mathrm{~Hz}, 2 \mathrm{H})$, 7.18 (t, $J=7.2 \mathrm{~Hz}, 1 \mathrm{H}$ ), 7.11 (dd, $J=8.4,1.2 \mathrm{~Hz}, 2 \mathrm{H}), 5.18(\mathrm{~d}, J=12.6 \mathrm{~Hz}$, 
1H), 5.12 (d, J=12.6 Hz, 1H), 5.07 (d, J=7.2 Hz, 1H), 4.40 (br, 1H), $2.67-$ $2.56(\mathrm{~m}, 2 \mathrm{H}), 2.18-2.12(\mathrm{~m}, 1 \mathrm{H}), 1.98-1.91(\mathrm{~m}, 1 \mathrm{H}), 1.45(\mathrm{~s}, 9 \mathrm{H})$.

${ }^{13} \mathrm{C}$ NMR (151 MHz, $\left.\mathrm{CDCl}_{3}\right): \delta 172.7,155.5,141.0,135.4,128.8,128.7$, 128.6, 128.54, 128.51, 126.3, 80.1, 67.2, 53.5, 34.6, 31.7, 28.5.

Spectral data are in accordance with those reported in the literature. ${ }^{21}$ 
5-Bromo-1-phenylpentane (52)<smiles>BrCCCCCc1ccccc1</smiles>

From isolated redox-active ester. Following General Procedure B, with SI-22 (47.8 mg, $0.1 \mathrm{mmol}), \mathrm{Fe}(\mathrm{acac})_{3}$ (7.1 mg, $\left.0.02 \mathrm{mmol}\right), \mathrm{dppBz}$ (10.7 mg, 0.024 $\mathrm{mmol})$ in toluene. $\mathrm{Ph}_{2} \mathrm{Zn}(0.15 \mathrm{mmol})$ was added at $25^{\circ} \mathrm{C}$. Product 52 was isolated by PTLC (hexanes) to afford $11.8 \mathrm{mg}(52 \%)$ as transparent oil.

From 6-bromohexanoic acid following General Procedure C: Activation was carried out with 6-bromohexanoic acid (19.5 mg, $0.1 \mathrm{mmol})$, HATU (38.0 mg, $0.1 \mathrm{mmol}$ ) and $\mathrm{Et}_{3} \mathrm{~N}(10.1 \mathrm{mg}, 0.1 \mathrm{mmol})$ in $0.2 \mathrm{~mL}$ of THF, followed by $\mathrm{Fe}(\mathrm{acac})_{3}(7.1 \mathrm{mg}, 0.020 \mathrm{mmol})$ and dppBz (10.7 mg, $\left.0.024 \mathrm{mmol}\right)$ in $0.5 \mathrm{~mL}$ of toluene. $\mathrm{Ph}_{2} \mathrm{Zn}(0.15 \mathrm{mmol})$ was added at r.t. Product $\mathbf{5 2}$ was isolated by PTLC (hexanes) to afford $16.3 \mathrm{mg}(72 \%)$ as yellowish oil.

Negishi-coupling using Ni-catalysis using in-situ generated RAE. Following General Procedure F, with 6-bromohexanoic acid (19.5 mg, $0.1 \mathrm{mmol}$ ), HATU (38.0 mg, $0.1 \mathrm{mmol}$ ), $\mathrm{Et}_{3} \mathrm{~N}$ (10.1 mg, $0.1 \mathrm{mmol}$ ), NiCl $2 \cdot \mathrm{glyme}(4.4 \mathrm{mg}, 0.02$ $\mathrm{mmol})$, di-tert-butylbipyridine (11 $\mathrm{mg}, 0.04 \mathrm{mmol}), \mathrm{PhZnCl}(0.3 \mathrm{mmol})$ at 25 ${ }^{\circ} \mathrm{C}$. Product 52 and $\mathbf{5 2 -} \mathbf{C l}(10.4 \mathrm{mg}, 54 \%)$ were isolated as mixture of isomers ( $\mathrm{Br}: \mathrm{Cl}=1: 2.9 \mathrm{~mol} / \mathrm{mol}$ ) by PTLC (hexanes). The mixture contained biphenyl as impurity $(9.4 \mathrm{mg})$. 


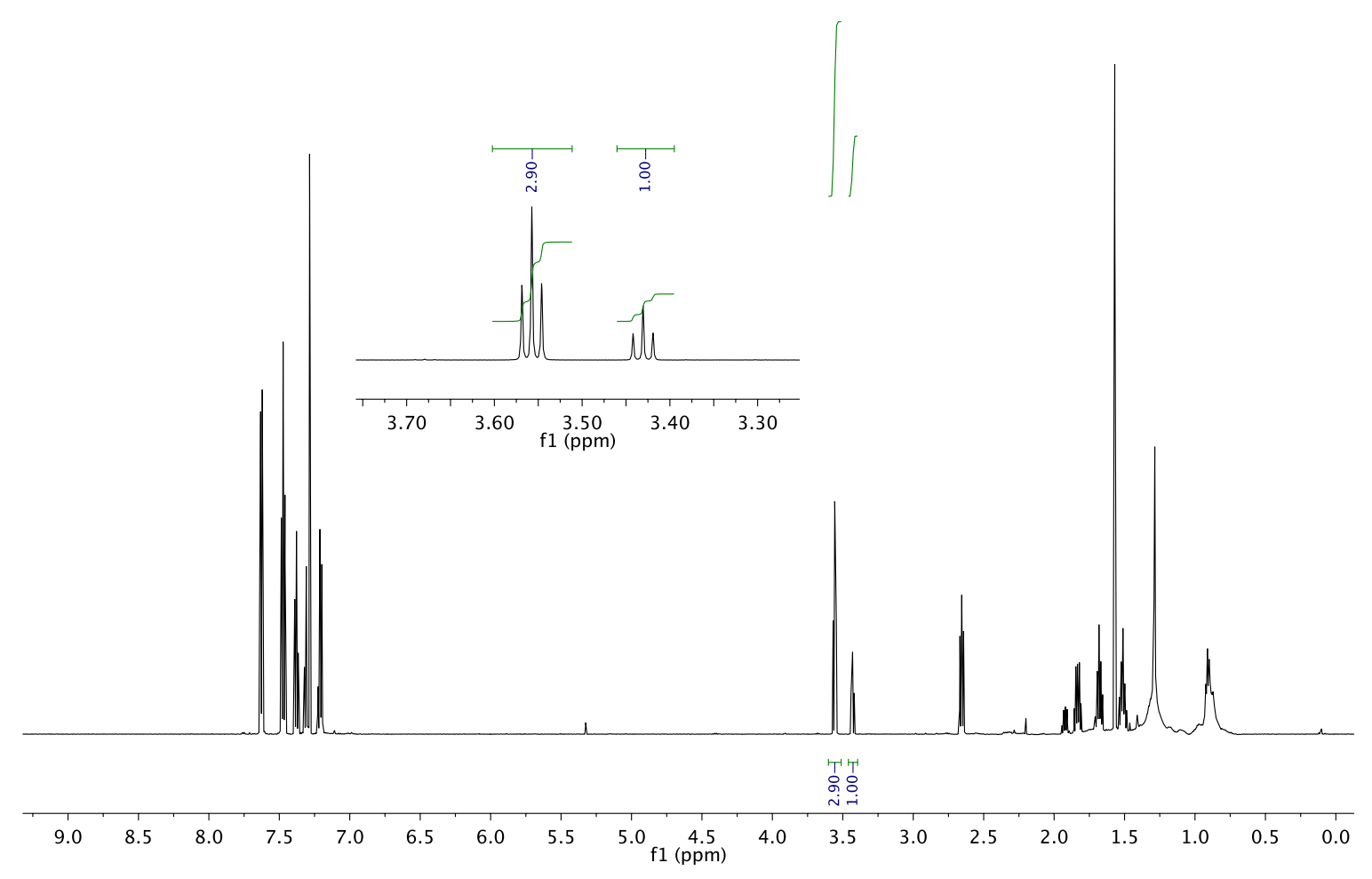

Negishi-coupling using Ni-catalysis. Following General Procedure E, with SI22 (47.8 mg, $0.1 \mathrm{mmol}$ ), $\mathrm{NiCl}_{2} \cdot$ glyme $(4.4 \mathrm{mg}, 0.02 \mathrm{mmol})$, di-tertbutylbipyridine $(11 \mathrm{mg}, 0.04 \mathrm{mmol}), \mathrm{PhZnCl}(0.3 \mathrm{mmol})$ at $25^{\circ} \mathrm{C}$. Product 52 and $52-\mathrm{Cl}(4.1 \mathrm{mg}, 22 \%)$ were isolated as mixture of isomers $(\mathrm{Br}: \mathrm{Cl}=1$ : $13.4 \mathrm{~mol} / \mathrm{mol}$ ) by PTLC (hexanes). The mixture contained biphenyl as impurity (4.4 mg). 


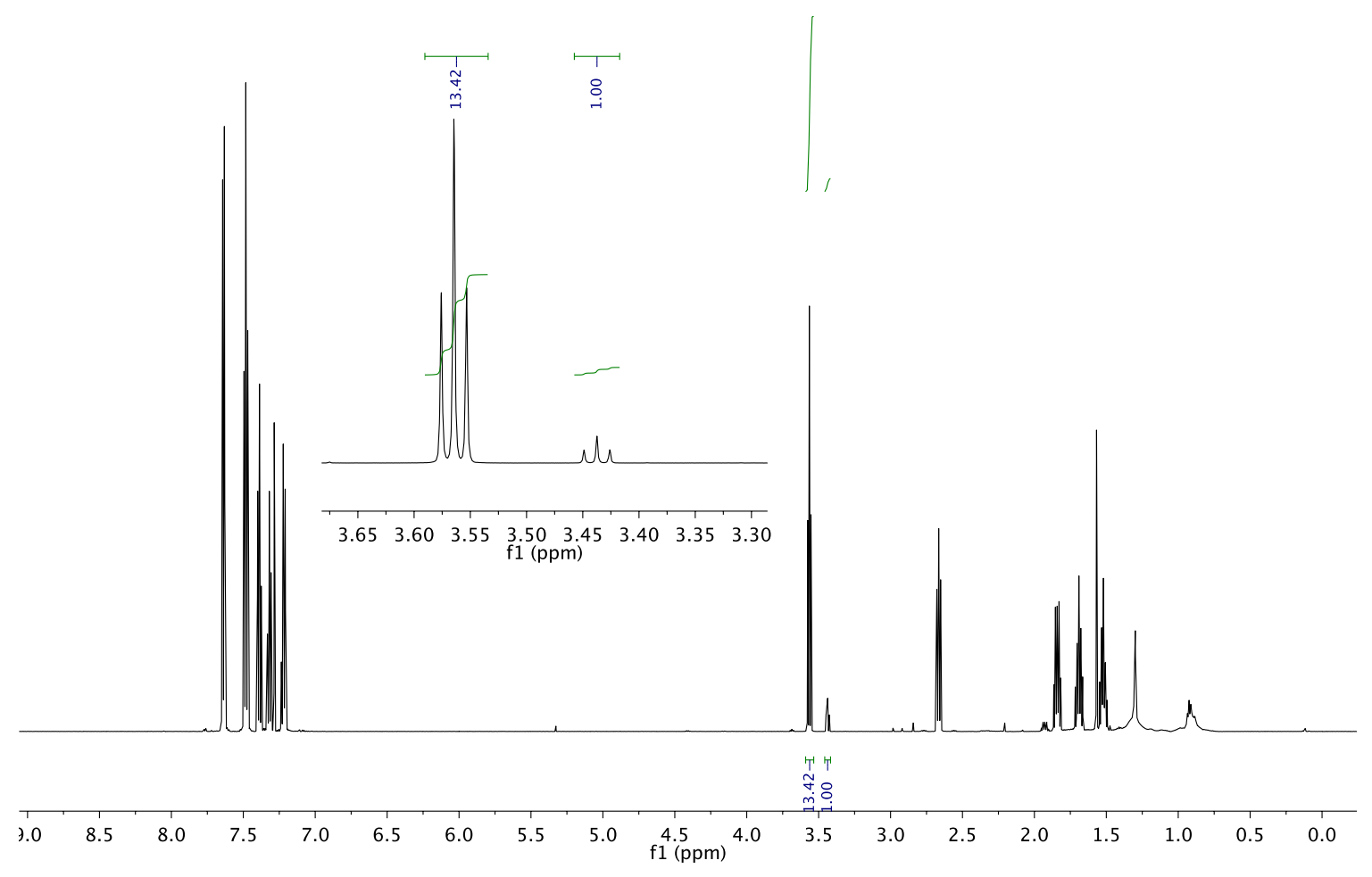

$\mathbf{R}_{f}=0.90$ (hexanes).

${ }^{1} \mathrm{H}$ NMR (600 MHz, $\left.\mathrm{CDCl}_{3}\right): \delta 7.29-7.27(\mathrm{~m}, 2 \mathrm{H}), 7.20-7.16(\mathrm{~m}, 3 \mathrm{H}), 3.40$ (t, $J=7.2 \mathrm{~Hz}, 2 \mathrm{H}), 2.63(\mathrm{t}, J=7.8 \mathrm{~Hz}, 1 \mathrm{H}), 1.92-1.87(\mathrm{~m}, 2 \mathrm{H}), 1.68-1.63$ $(\mathrm{m}, 2 \mathrm{H}), 1.51-1.46(\mathrm{~m}, 2 \mathrm{H})$.

${ }^{13} \mathrm{C}$ NMR (151 MHz, $\left.\mathrm{CDCl}_{3}\right): \delta 142.5,128.5,128.5,125.9,35.9,33.9,32.8$, 30.8, 28.0.

Spectral data are in accordance with those reported in the literature. ${ }^{22}$ 


\section{Procedure for a mock-medicinal chemistry gram-scale Fe-catalyzed}

Negishi cross-coupling with redox-active esters generated in situ.

In a $50 \mathrm{~mL}$ round-bottomed flask, 1-tosylpiperidine-4-carboxylic acid 46 (1.42 $\mathrm{g}, 5.0 \mathrm{mmol})$, HATU $(1.90 \mathrm{~g}, 5.0 \mathrm{mmol})$ were weighed. The flask was equipped with a rubber septum, evacuated and refilled with $\operatorname{Ar} 3$ times. Then, $\mathrm{Et}_{3} \mathrm{~N}(0.69 \mathrm{~mL}, 5.0 \mathrm{mmol})$ followed by THF $(10 \mathrm{~mL})$ were added via syringe under Ar. The mixture was stirred for $2 \mathrm{~h}$ at room temperature (usually, the solution becomes clear after this time). In a separate round-bottomed flask, $\mathrm{Fe}(\mathrm{acac})_{3}(177 \mathrm{mg}, 0.5 \mathrm{mmol})$ and dppBz (446 mg, $\left.1.0 \mathrm{mmol}\right)$ were weighed. The flask was evacuated and backfilled with Ar 3 times. Then, THF (15 mL) was added to form a red solution. The solution was stirred until all solids were soluble (20 $\mathrm{min}$ ). At this point, the mixture was transferred to the redox-active ester flask in one portion at $0{ }^{\circ} \mathrm{C}$, followed by the addition of $\mathrm{Ph}_{2} \mathrm{Zn}(36.8 \mathrm{~mL}$, $0.34 \mathrm{M}, 12.5 \mathrm{mmol}, 2.5$ equiv.) via syringe in one portion at the same temperature. The mixture was warmed to room temperature immediately after the addition of the $\mathrm{Ph}_{2} \mathrm{Zn}$ and stirred for $1 \mathrm{~h}$. The mixture was quenched with $1 \mathrm{~N} \mathrm{HCl}$ and diluted with diethyl ether. The aqueous layer was extracted with additional diethyl ether ( 3 times) and the combined organic layers were washed with $3 \mathrm{M} \mathrm{NaOH}$, separated dried over $\mathrm{Na}_{2} \mathrm{SO}_{4}$ anhydrous, filtered and evaporated to dryness. Pure product 13 (1.08 g, 69\% yield) was obtained after column chromatography (hexanes:ethyl acetate 8:2). 


\subsection{Graphical Guide for the Fe-catalyzed Negishi coupling at gram scale.}
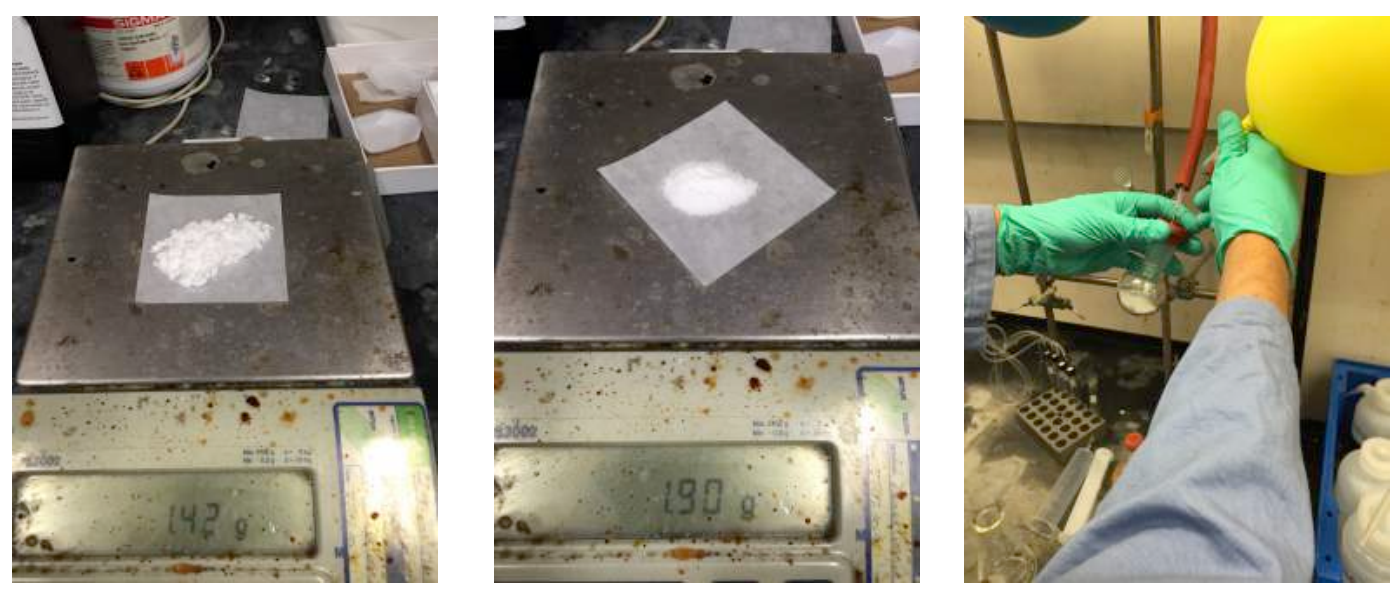

Left: 4-tosylpiperidine-1-carboxylic acid weight. Center: HATU weight. Right: evacuation and backfilling with Ar of the HATU/carboxylic acid mixture.
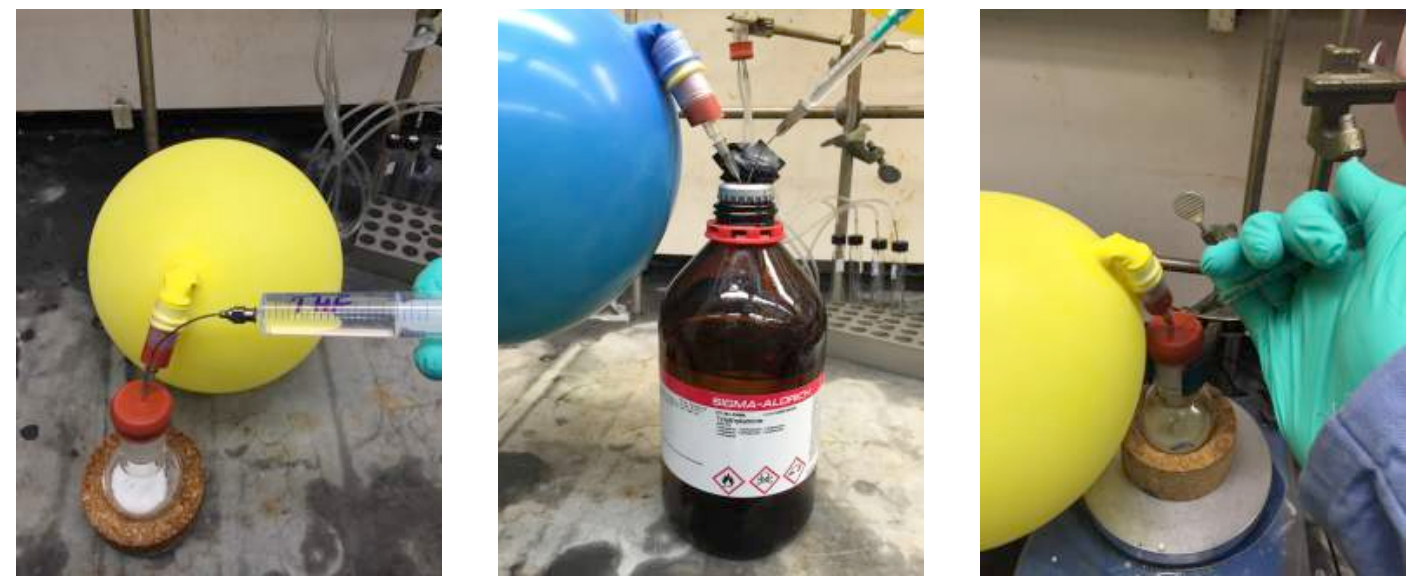

Left: addition of THF. Center: triethylamine anhydrous. Right: addition of triethylamine.
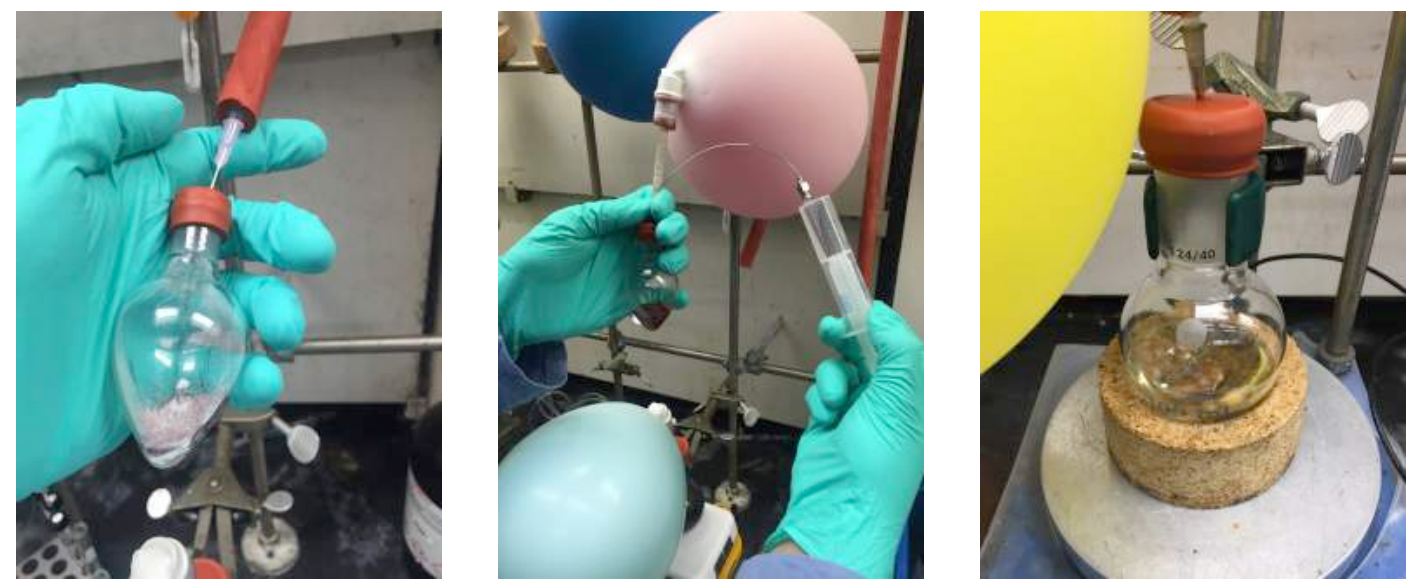

Left: $\mathrm{Fe}(\mathrm{acac})_{3}$ and $\mathrm{dppBz}$ in a flask. Evacuated and backfilled with Ar. Center: addition of THF. Right: redox-active ester after $2 \mathrm{~h}$ at r.t. 

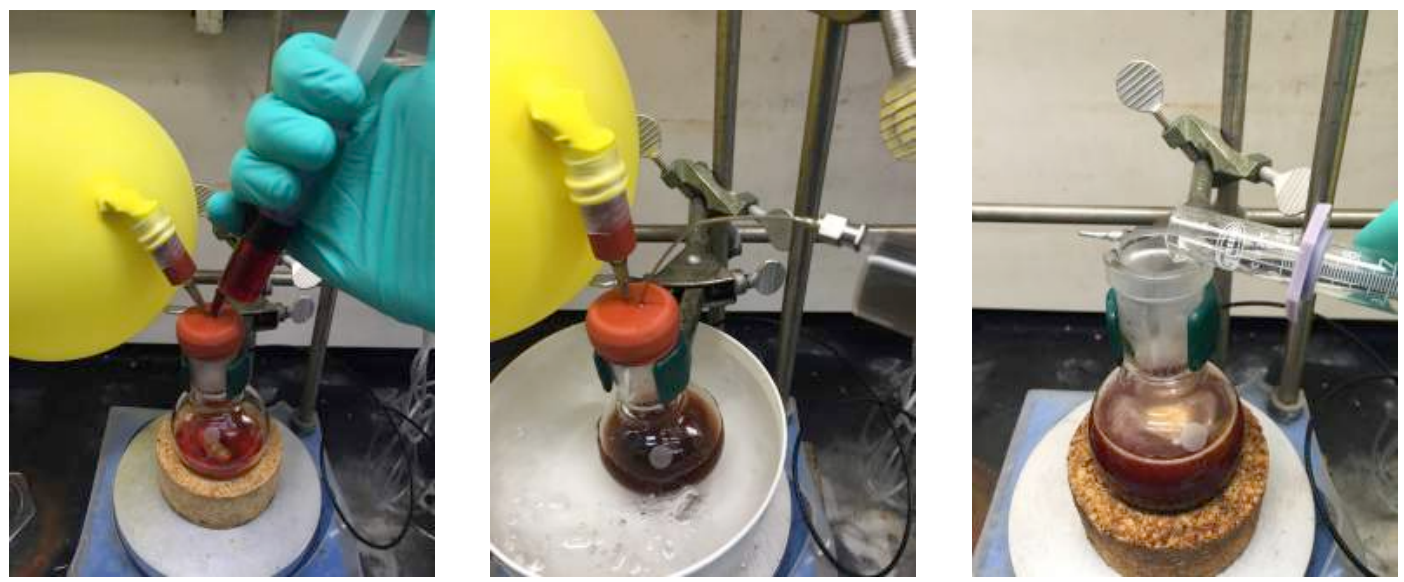

Left: addition of $\mathrm{Fe} /$ ligand solution. Center: addition of the organozinc at $0{ }^{\circ} \mathrm{C}$. Right: quench the reaction after $1 \mathrm{~h}$ with $1 \mathrm{M} \mathrm{HCl}$.
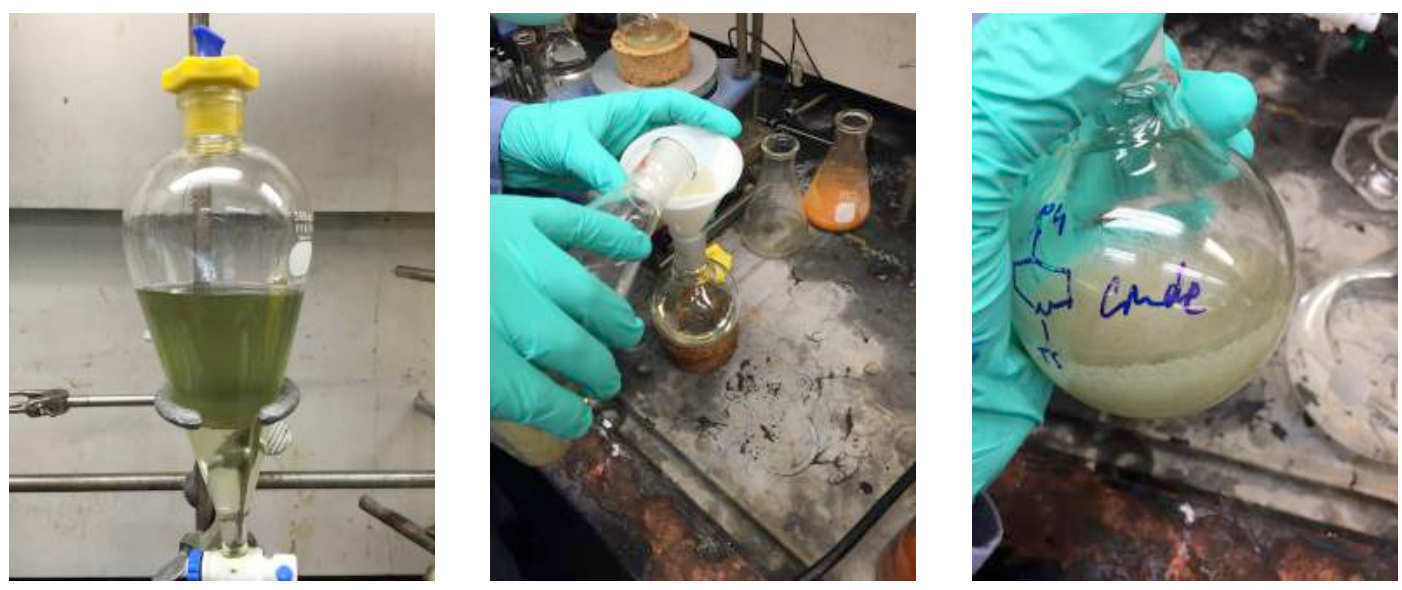

Left: dilution with diethyl ether or ethyl acetate and extract. Center: filtration of the drying agent. Right: crude reaction mixture after solvent evaporation.
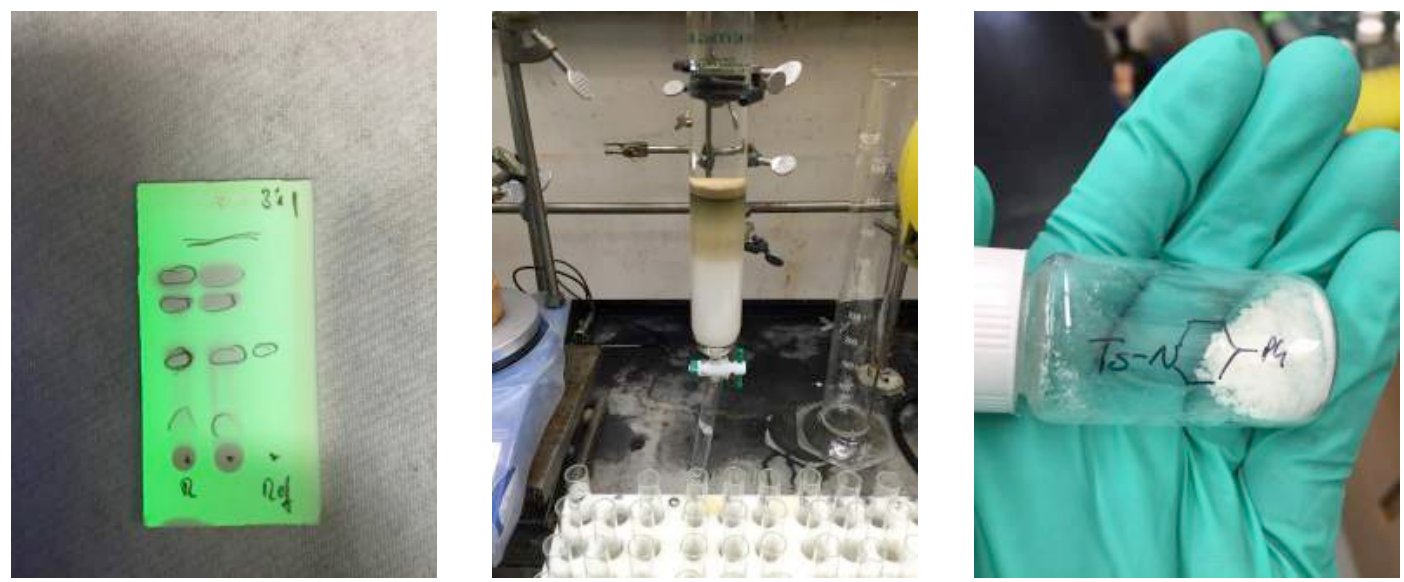

Left: TLC plate of the reaction (left: reaction mixture; center: cross-spot; right: pure material).

Center: column chromatography. Right: isolated pure material. 


\section{Procedure for the mock-process chemistry scale-up and isolation of the Fe-catalyzed Negishi cross-coupling.}

In a $100 \mathrm{~mL}$ 3-necked flask equipped with a double manifold and a temperature sensor, 1-tosylpiperidine-4-carboxylic acid 46 (1.42 g, $5.0 \mathrm{mmol}$ ), HATU $(1.90 \mathrm{~g}, 5.0 \mathrm{mmol})$ were weighed. The flask was equipped with a rubber septum, evacuated and refilled with $\mathrm{Ar} 3$ times. Then, $\mathrm{Et}_{3} \mathrm{~N}(0.69 \mathrm{~mL}$, $5.0 \mathrm{mmol}$ ) followed by THF (10 mL) were added via syringe under Ar. The mixture was stirred for 2 hours at room temperature (the solution becomes clear after this time). In a separate 2-necked flask equipped with a double manifold, $\mathrm{Fe}(\mathrm{acac})_{3}(18 \mathrm{mg}, 0.05 \mathrm{mmol})$ and dppBz $(27 \mathrm{mg}, 0.06 \mathrm{mmol})$ were weighed. The flask was evacuated and backfilled with $\operatorname{Ar} 3$ times. Then, THF $(15 \mathrm{~mL})$ was added to form a red solution. The solution was stirred until all solids were soluble (10 $\mathrm{min})$. At this point, the mixture was transferred to the redox-active ester vial in one portion at the same temperature. The mixture was cooled at $0{ }^{\circ} \mathrm{C}$, followed by the addition of $\mathrm{Ph}_{2} \mathrm{Zn}(37.9 \mathrm{~mL}, 0.33 \mathrm{M}, 12.5$ $\mathrm{mmol}, 2.5$ equiv.) via syringe in one portion at the same temperature (internal temperature rose from 1.5 to $19.0{ }^{\circ} \mathrm{C}$ after addition of $\mathrm{Ph}_{2} \mathrm{Zn}$ ). The mixture was stirred at room temperature for $1 \mathrm{~h}$. The mixture was quenched with $3 \mathrm{M}$ $\mathrm{HCl}\left(20 \mathrm{~mL}\right.$ ) after cooling to $3.4{ }^{\circ} \mathrm{C}$ and THF was removed in vacuo. The aqueous layer was extracted with toluene $(25 \mathrm{~mL})$, the organic layers were washed with water $(20 \mathrm{~mL} \times 2)$ and $2 \mathrm{M} \mathrm{NaOH}(20 \mathrm{~mL})$, and evaporated to 10 $\mathrm{mL}$. To the residue, 2-PrOH $(20 \mathrm{~mL})$ was added dropwise and evaporated to $10 \mathrm{~mL}$ (The process of addition of 2-PrOH and evaporation was repeated 2 times). To the resulting mixture 2-PrOH $(15 \mathrm{~mL})$ was added at $50{ }^{\circ} \mathrm{C}$ and stirred for $1 \mathrm{~h}$ at the same temperature. After this time, stirred at room temperature for $30 \mathrm{~min}$. Then, filtered through a sintered funnel followed by a 2-PrOH (10 mL) wash and dry under vacuum. Product 13 was obtained as a white crystalline solid ( $0.96 \mathrm{~g}, 61 \%$ yield, $99 \mathrm{wt} \%$ purity). 


\subsection{Graphical guide for the mock-process chemistry scale-up and isolation of the Fe-catalyzed Negishi cross-coupling.}

For graphical support for carrying out the reaction on large scale, see Section 12.1.
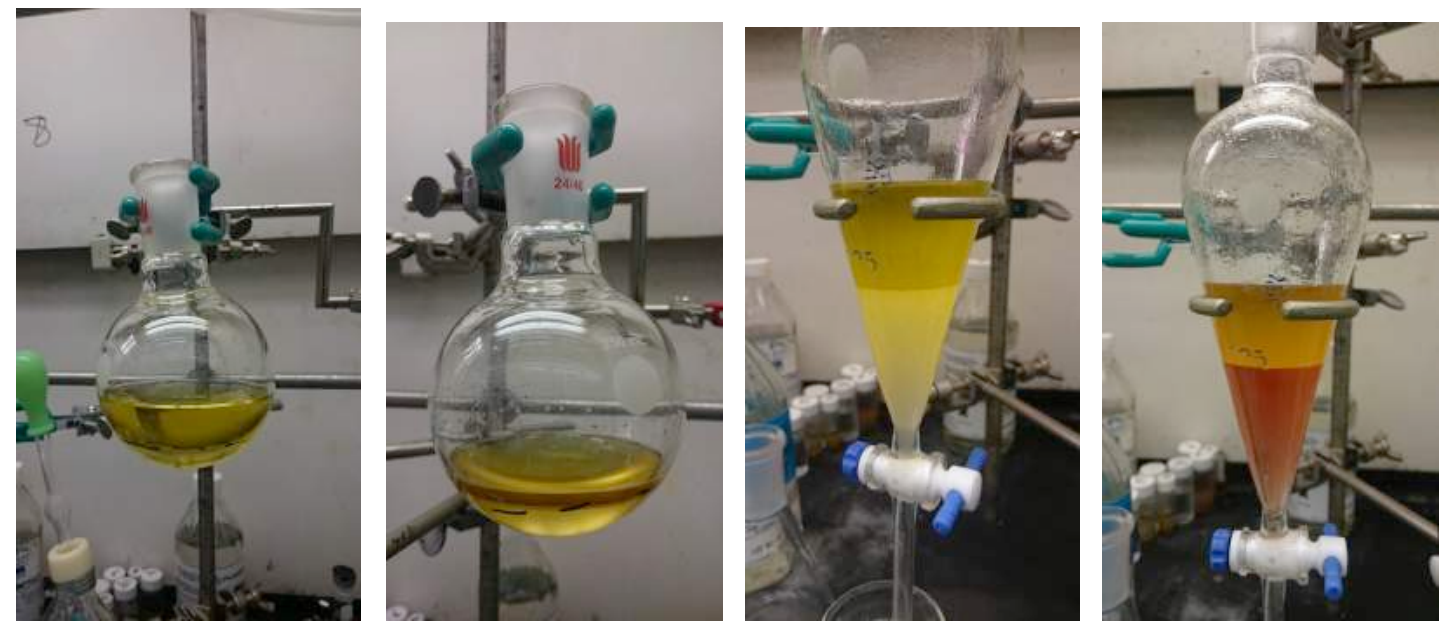

Left: reaction mixture after quench with $\mathrm{HCl}$. Center-left: after removal of THF. Center-right: addition of toluene and extraction. Right: initial wash with water $(\mathrm{pH}=0)$.
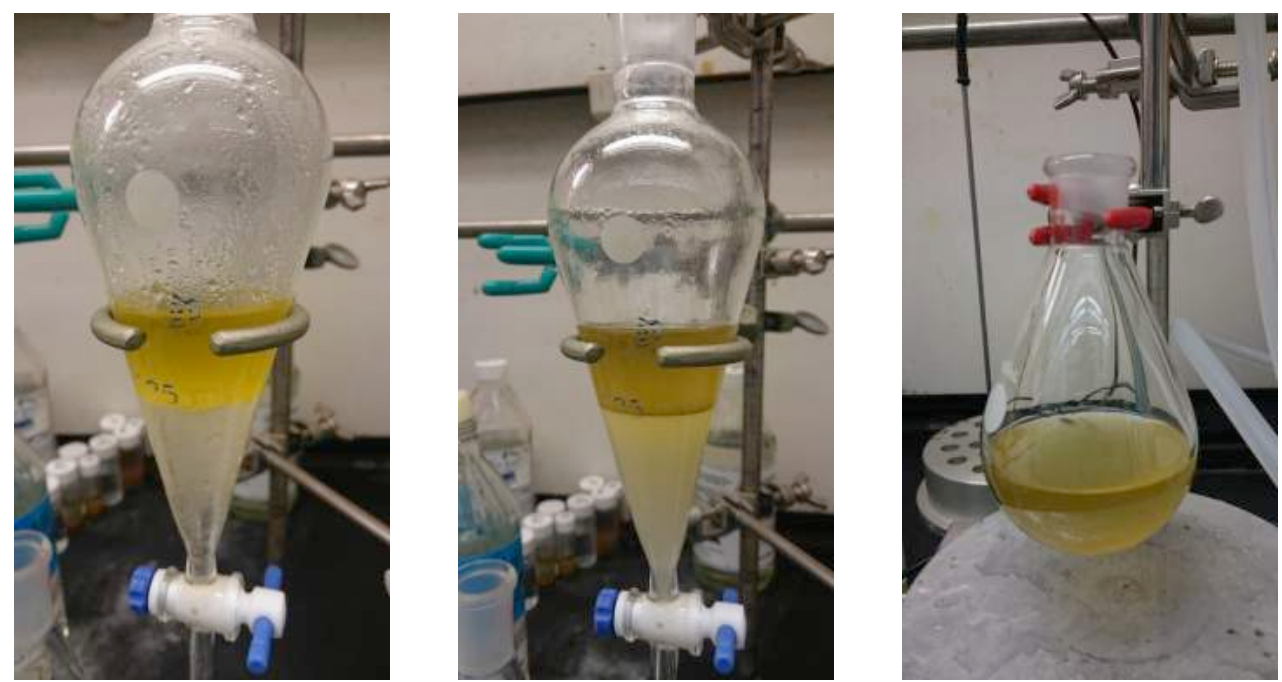

Left: second wash with water $(\mathrm{pH}=3)$. Center: wash with $2 \mathrm{M} \mathrm{NaOH}$. Right: organic solution after separation of aqueous layer. 


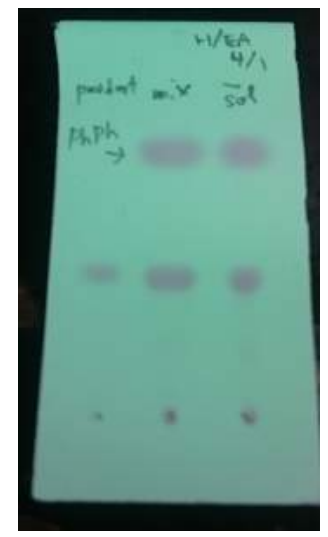

TLC plate (left: pure material; center: cross-spot; right: organic solution after washing).
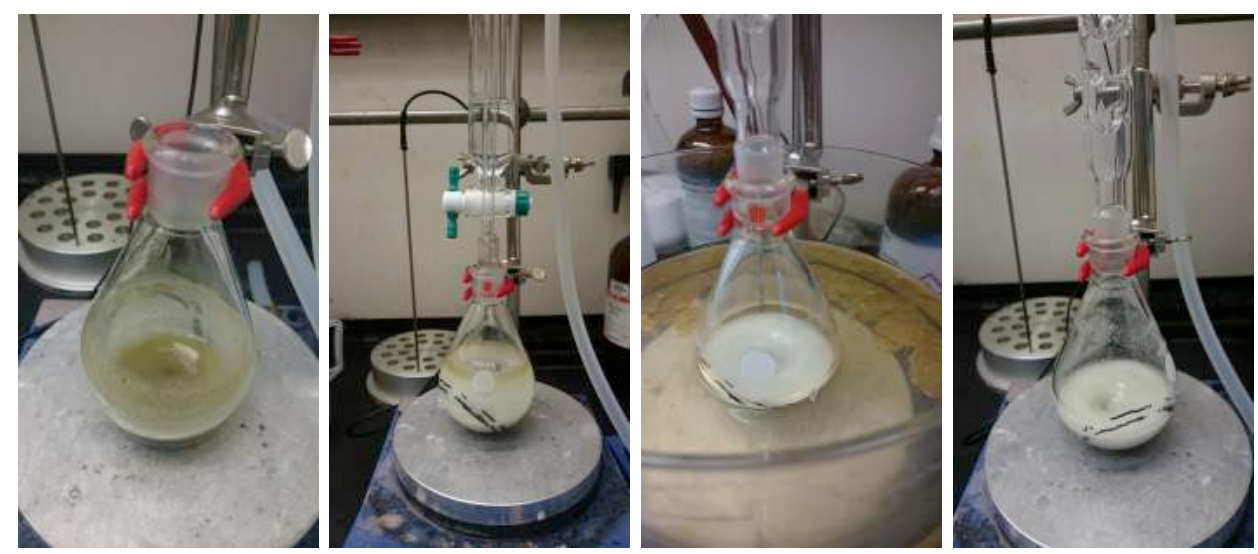

Left: concentration to $10 \mathrm{~mL}$ of toluene. Center-left: dropwise addition of 2-PrOH using an addition funnel. Center-right: stirring the system at $50^{\circ} \mathrm{C}$. Right: after cooling down and crystallization.
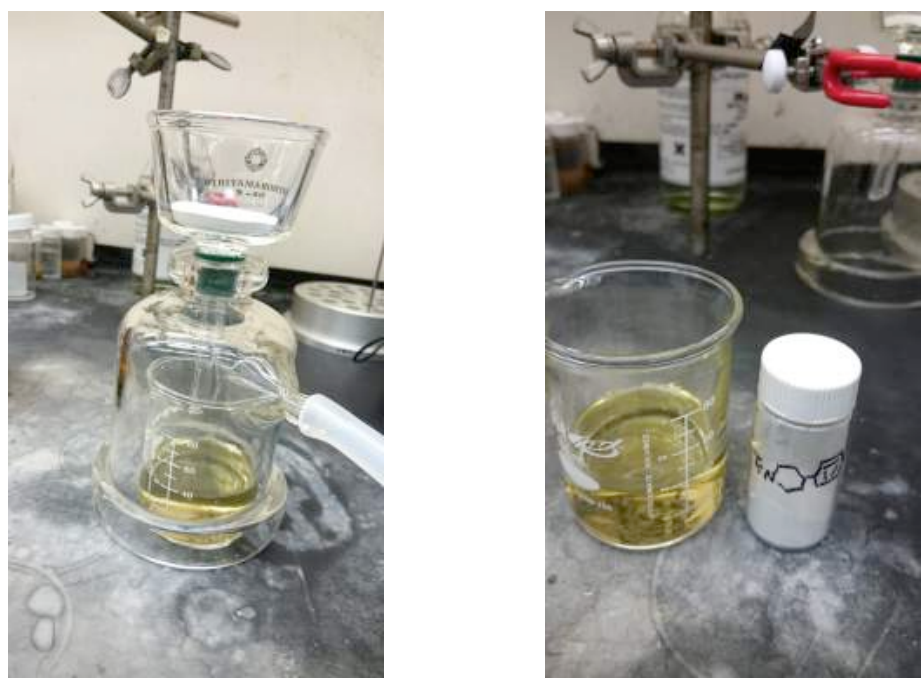

Left: filtration using a sintered funnel. Right: isolated crystalline material and mother liquor. 


\section{Troubleshooting \& FAQ}

\section{When do I choose Fe- over Ni-catalysis?}

Fe-catalysis has proven particularly effective for the arylation of tertiary bridge-head carbons, which are inaccessable using Ni-catalysis. In the Fecatalyzed reaction, HATU or HBTU activation afforded comparable yields to using isolated RAE. In contrast, in the case of Ni-catalyzed arylation, activation using HATU or HBTU provided workable yields only for $\alpha$ heteroatom containing acids, e.g. proline. Moreover, the kinetic profile established faster reaction rates for Fe over $\mathrm{Ni}$.

What can I do if I observe significant amounts of H-atom abstraction in the reaction.

We observed that the use of toluene as a co-solvent limited the formation of by-products originating from $\mathrm{H}$-atom abstraction by the alkyl radical generated. These by-products were primarily observed in reactions of tertiary carboxylic acids.

\section{Is the HATU-protocol or the protocol using isolated redox-active esters best for my purpose?}

Typically, primary and secondary carboxylic acids performed equally well under conditions. However, in certain cases either HATU or isolated RAE showed superior performance. We recommend to the use of HATU as the standard procedure. If yields are not satisfactory it might be worth looking into the use of isolated RAE.

In couplings of certain tertiary carboxylic acids (e.g. adamantane) the use of HATU led to deminished yields accompanied by formation of Minisci byproducts. Switching to HBTU restored the yield of cross-coupling and suppressed the formation of these by-products. 


\section{Which conditions should I choose for my particular reaction?}

Conditions differ depending on whether isolated ester or in situ activation will be used, and on the type of RAE (primary, secondary, tertiary or amino acid). We have devised a decision tree to aid practitioners in selecting optimal Fecatalyzed cross-coupling conditions for their particular problem (see Chapter 10)

\section{Can I employ any tertiary carboxylic acid in this reaction?}

Currently, our Fe-protocol is limited to bridge-head carboxylic acids and 1phenylcyclopropyl-1-carboxylic acids. Compounds structurally similar to pivalic acid or 1-methylcyclohexyl-1-carboxylic acid were found to be incompetent reaction partners.

\section{Is it necessary to wait for two hours for the HATU activation?}

Compared to the traditional activation of carboxylic acids in DMF, this process is relatively slow in THF. Furthermore low solubility of some carboxylic acids in THF can lead to prolonged activation times. Across a wide range of different carboxylic acids we have found that two hours reaction time reliably afford full conversion to the RAE. This process can be accelerated by gentle heating $\left(50^{\circ} \mathrm{C}\right)$ of the activation reaction.

\section{How important is the solvent quality?}

All our experiments were performed with high-quality THF free of stabilizers. We have previously found that solvents containing stabilizers can completely shut down cross-coupling of RAE. Hence, we highly recommend avoiding stabilized solvents. 


\section{Can I use other Fe-sources in this reaction?}

We have found, that other iron (III) sources are also competent in this reaction. However, we recommend the use of $\mathrm{Fe}(\mathrm{acac})_{3}$, anhydrous $\mathrm{FeCl}_{3}$ or $\mathrm{FeCl}_{3}$ hydrate for best performance.

\section{How long do I have to stir my reaction to reach full conversion?}

The reactions were found to be extremely fast. Our kinetic experiment showed, that full conversion was already reached after 5 minutes. We ran our reactions with a standardized reaction time of one hour to ensure reproducibility across the wide range of acids tested. In our experience, longer reaction times do not affect the outcome of the reactions.

\section{Can I use Fe-catalysis for the coupling of two primary centers?}

Unfortunately, at the current stage coupling of two primary centers is not viable. We have observed the formation of large amounts $\beta$-hydride elimination of both coupling partners. We highly recommend the use of our complementary method using Ni-catalysis.

\section{Which procedure should I follow to prepare the dppBz ligand?}

We have performed experiments with either commercial or in-house prepared $\mathrm{dppBz}$ without noticing any differences in performance. For the preparation of dppBz, see Ref 23.

\section{How long do I have to stir the Fe/ligand solution?}

We have stirred the solution at least for 5 minutes. We suggest stirring until all components are dissolved. In cases were $40 \mathrm{~mol} \%$ of ligand were used a small quantity of solid remained undissolved. 
Can I syringe-pump the diarylzinc solution?

Rapid addition of the arylzinc reagent is critical for the success of the reaction. We do not recommend the use of syringe pumps.

\section{Can I use arylzinc chloride in place of diarylzinc?}

For primary and secondary carboxylic acids, arylzinc chlorides are competent coupling partners in the Fe-catalyzed couplings, albeit in slightly diminished yields. Our attempts to couple tertiary carboxylic acids afforded only trace amounts of product.

Where can I find addition information and a comprehensive visual guide for the preparation of RAE and HATU activation?

In the three publications preceeding this report we have given detailed information on the preparation of RAE, see Ref 1 and'2.

Which by-products should I expect in this reaction?

The main by-product originating from the arylzinc reagent are its homodimerization product along with protodemetalation. The major side product formed from RAE is the hydrolysis product, which in turn is the starting carboxylic acid.

Other by-products observed in variable amounts are $\beta$-hydride elimination of the RAE and hydrogen abstraction by the alkyl radical. 
15. Mechanistic investigation.<smiles>O=C(CC1CC1)ON1C(=O)c2c(Cl)c(Cl)c(Cl)c(Cl)c2C1=O</smiles><smiles>C=CCCc1ccccc1</smiles>

$57 \%$ GC yield exclusive isomer observed

The reaction was carried out following the General Procedure B with SI-20 (38 $\mathrm{mg}, 0.1 \mathrm{mmol}$ ). After $1 \mathrm{~h}$, dodecane (internal standard, $0.1 \mathrm{mmol}, 23 \mu \mathrm{L}$ ) was then added to the mixture and an aliquot was analyzed by GC-FID. Correction factor for SI-36 versus dodecane is 0.98 .

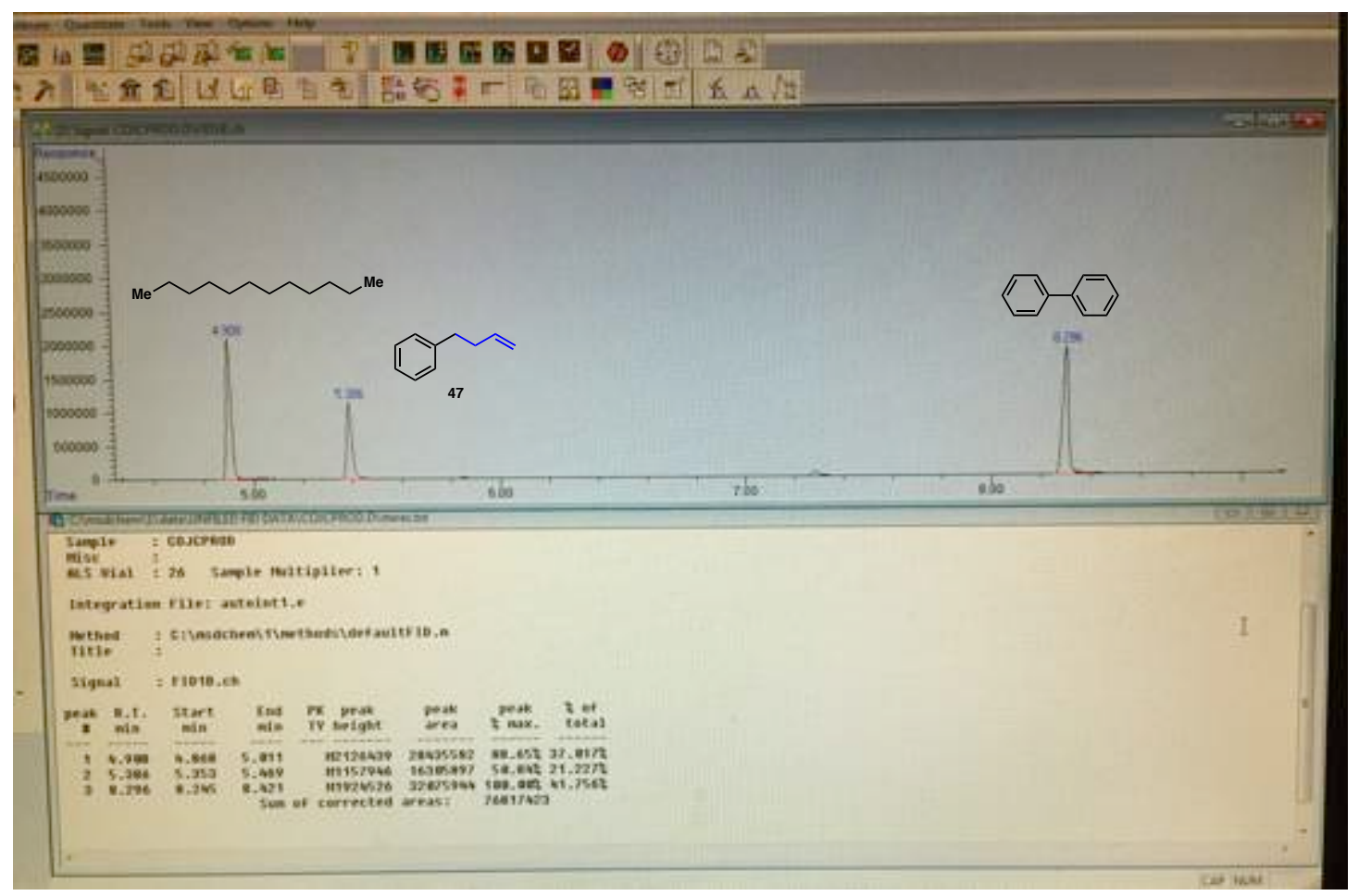

GC-FID of the crude reaction mixture. 


\section{Iterative diarylation of cubane carboxylic acid.}

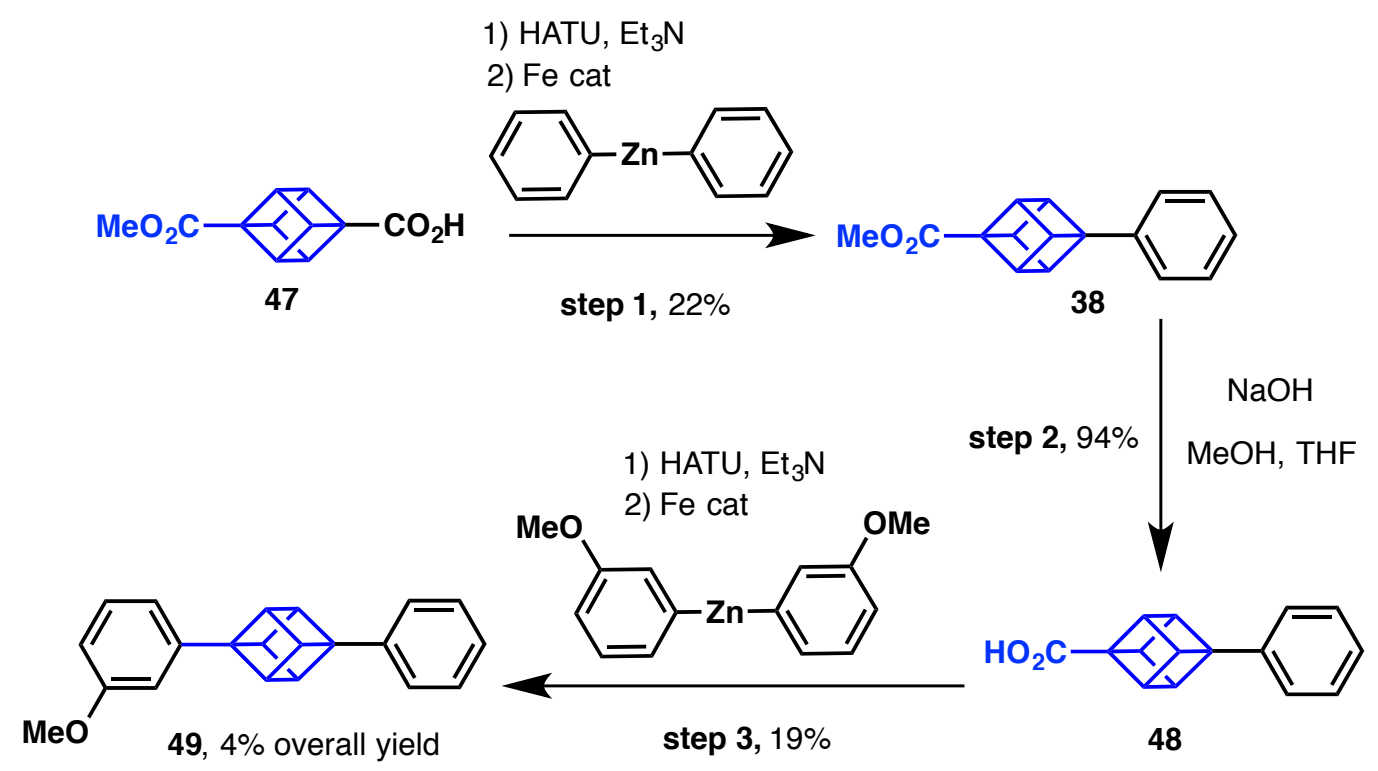

Step 1. In a $100 \mathrm{~mL}$ round bottom flask, 4-(methoxycarbonyl)cubane-1carboxylic acid (47) (825 mg, $4.0 \mathrm{mmol})$, HATU (1.52 g, $4.0 \mathrm{mmol}$ ) were weighed. The flask was sealed, evacuated and refilled with Ar 3 times. Then, $\mathrm{Et}_{3} \mathrm{~N}(0.55 \mathrm{~mL}, 4.0 \mathrm{mmol})$ followed by THF ( $8 \mathrm{~mL}$ ) were added via syringe under Ar. The mixture was stirred for 2 hours at room temperature. In another $25 \mathrm{~mL}$ round bottom flask, $\mathrm{Fe}(\mathrm{acac})_{3}(283 \mathrm{mg}, 0.8 \mathrm{mmol})$ and dppBz $(714 \mathrm{mg}$, $1.6 \mathrm{mmol}$ ) were weighed. The vial evacuated and backfilled with $\operatorname{Ar} 3$ times. Then, THF (12 mL) was added to form a red solution. The solution was stirred until all solids were soluble (10 $\mathrm{min})$. At this point, the mixture was transferred to the redox-active ester vial in one portion at $0{ }^{\circ} \mathrm{C}$, followed by the addition of $\mathrm{Ph}_{2} \mathrm{Zn}$ (23.8 mL, $0.42 \mathrm{M}, 10.0 \mathrm{mmol}, 2.5$ equiv.) via syringe in one portion. The mixture was stirred for $1 \mathrm{~h}$ at r.t. before quench with $1 \mathrm{~N} \mathrm{HCl}$ and diluted with diethyl ether and extracted (3 times). The organic layer was separated and dried over $\mathrm{Na}_{2} \mathrm{SO}_{4}$ anhydrous, filtered and evaporated to dryness. Pure product 38 (215 $\mathrm{mg}, 22 \%)$ was obtained after column chromatography hexanes:ethyl acetate 15:1 and subsequent crystallization from $\mathrm{MeOH}: \mathrm{H}_{2} \mathrm{O}$.

Step 2. In a $25 \mathrm{~mL}$ round bottom flask, ester $38(97 \mathrm{mg}, 0.41 \mathrm{mmol})$ was dissolved in THF (4 mL). Then, a solution of $\mathrm{NaOH}$ in $\mathrm{MeOH}(0.49 \mathrm{~mL}, 1 \mathrm{M}$, 
0.49 mmol, 1.2 equiv.) was added. The mixture was stirred at $25^{\circ} \mathrm{C}$ for $16 \mathrm{~h}$ before evaporate to dryness. The crude was then redissolved in $\mathrm{H}_{2} \mathrm{O}$, washed with $\mathrm{CH}_{2} \mathrm{Cl}_{2}$ (2 times), and the $\mathrm{pH}$ adjusted to $1-2$ with $2 \mathrm{M} \mathrm{HCl}$. The mixture was further extracted with $\mathrm{CH}_{2} \mathrm{Cl}_{2}$, dried with $\mathrm{MgSO}_{4}$ anhydrous, filtered and evaporated to afford pure acid 48 (86 $\mathrm{mg}, 94 \%)$ without further purification. Spectroscopic data for $\mathbf{4 8}$ matches those reported in the literature. ${ }^{24}$

Step 3. Compound 49 was prepared following General Procedure C, using 48 (67 mg, $0.3 \mathrm{mmol}), \mathrm{Fe}(\mathrm{acac})_{3}$ (21 mg, $\left.0.06 \mathrm{mmol}\right), \mathrm{dppBz}$ (54 mg, $0.12 \mathrm{mmol}$ ), bis(3-methoxyphenyl)zinc (2.27 mL, $0.33 \mathrm{M}, 0.75 \mathrm{mmol}, 2.5$ equiv.). Compound 49 was obtained in pure form (16.5 mg, 19\%) after two PTLC (hexanes:toluene 4:1 and hexanes: $\mathrm{CH}_{2} \mathrm{Cl}_{2}$ 4:1).

\section{4-Phenylcubane-1-carboxylic acid (48). ${ }^{25}$}

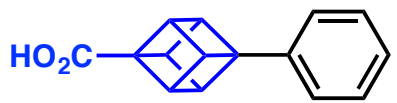

48

${ }^{1} \mathrm{H}$ NMR (600 MHz, $\left.\mathrm{CDCl}_{3}\right) \delta 7.39-7.35(\mathrm{~m}, 2 \mathrm{H}), 7.25-7.19(\mathrm{~m}, 3 \mathrm{H}), 4.35-$ $4.25(\mathrm{~m}, 3 \mathrm{H}), 4.25-4.14(\mathrm{~m}, 3 \mathrm{H})$.

${ }^{13} \mathrm{C}$ NMR (151 MHz, $\left.\mathrm{CDCl}_{3}\right) \delta 178.3,142.0,128.6,126.4,124.9,77.4,77.2$, $76.9,60.4,56.3,48.9,46.2$.

\section{1-(3-Methoxyphenyl)-4-phenylcubane (49).}

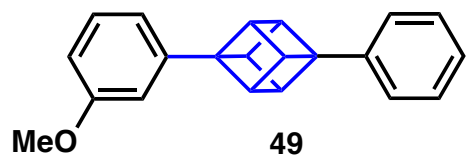

m.p. $79-81^{\circ} \mathrm{C}$

$\mathbf{R}_{f}=0.56$ (hexanes/ethyl acetate 15:1)

${ }^{1} \mathbf{H}$ NMR (600 MHz, $\mathrm{CDCl}_{3}$ ) $\delta 7.41-7.36$ (m, 2H), 7.31 (ddd, $J=8.1,7.5,0.5$ $\mathrm{Hz}, 1 \mathrm{H}), 7.30-7.25$ (m, 2H), $7.25-7.19(\mathrm{~m}, 1 \mathrm{H}), 6.87$ (ddd, J = 7.5, 1.5, 0.9 $\mathrm{Hz}, 1 \mathrm{H}), 6.80$ (dd, J=2.6, $1.5 \mathrm{~Hz}, 1 \mathrm{H}), 6.77$ (ddd, $J=8.2,2.6,1.0 \mathrm{~Hz}, 1 \mathrm{H}$ ), $4.18-4.13(\mathrm{~m}, 6 \mathrm{H}), 3.85(\mathrm{~s}, 3 \mathrm{H})$. 
${ }^{13} \mathrm{C}$ NMR (151 MHz, $\left.\mathrm{CDCl}_{3}\right) \delta 160.0,145.0,143.2,129.6,128.6,126.0$, $124.9,117.3,111.4,110.6,77.4,77.2,76.9,61.03,61.98,55.4,47.9$

HRMS (ESI-TOF) calc'd for $\mathrm{C}_{21} \mathrm{H}_{19} \mathrm{O}[\mathrm{M}+\mathrm{H}]^{+} 287.1430$ found 287.1432.

1-Phenyl-4-(3-(trifluoromethyl)phenyl)cubane (50)

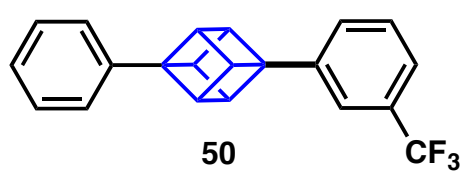

Compound $\mathbf{5 0}$ was prepared via the above mentioned three-step procedure, only differing in step 3.

Following General Procedure C, using $48(22 \mathrm{mg}, 70 \%$ wt by NMR, 0.07 $\mathrm{mmol}), \mathrm{Fe}(\mathrm{acac})_{3}(7 \mathrm{mg}, 0.02 \mathrm{mmol}), \mathrm{dppBz}$ (18 mg, $\left.0.04 \mathrm{mmol}\right)$, bis(3benzotrifluoride)zinc ( $1.32 \mathrm{~mL}, 0.19 \mathrm{M}, 0.25 \mathrm{mmol}, 2.5$ equiv.). Compound 50 was obtained in pure form $(4.0 \mathrm{mg}, 18 \%)$ after two PTLC (hexanes and $\mathrm{CH}_{2} \mathrm{Cl}_{2}$ ).

m.p. $60-62^{\circ} \mathrm{C}$.

${ }^{1} \mathrm{H}-\mathrm{NMR}\left(600 \mathrm{MHz}, \mathrm{CDCl}_{3}\right)$ ठ $7.55-7.45(\mathrm{~m}, 4 \mathrm{H}), 7.44-7.38(\mathrm{~m}, 2 \mathrm{H}), 7.31-$ $7.28(\mathrm{~m}, 2 \mathrm{H}), 7.27-7.23(\mathrm{~m}, 1 \mathrm{H}), 4.21(\mathrm{~s}, 6 \mathrm{H})$.

${ }^{13} \mathrm{C}-\mathrm{NMR}\left(151 \mathrm{MHz}, \mathrm{CDCl}_{3}\right.$ ) $\delta$ 143.5, 142.3, 130.3 (q, $J=31.8 \mathrm{~Hz}$ ), 128.4, 128.0, 127.8, 125.6, 123.0, 123.9 (q, $J=272.4 \mathrm{~Hz}), 122.2$ (q, $J=3.4 \mathrm{~Hz}$ ), $121.2(q, J=3.8 \mathrm{~Hz}), 60.5,60.0,47.3,47.2$.

${ }^{19} \mathrm{~F}-\mathrm{NMR}\left(376 \mathrm{MHz}, \mathrm{CDCl}_{3}\right) \delta-62.8$. 


\subsection{Graphical guide for the diarylation of cubane carboxylic acid.}

\section{Step 1. First arylation.}
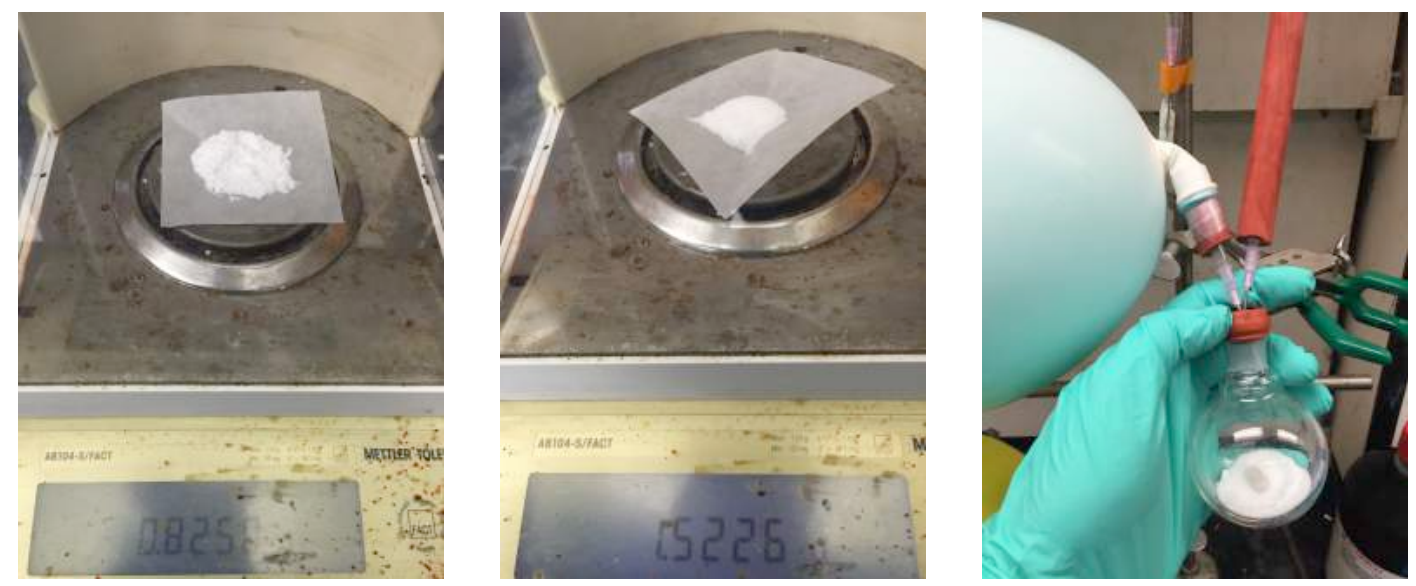

Left: carboxylic acid weight. Center: HATU weight. Right: carboxylic acid and HATU in a flask, evacuated and backfilled with Ar.
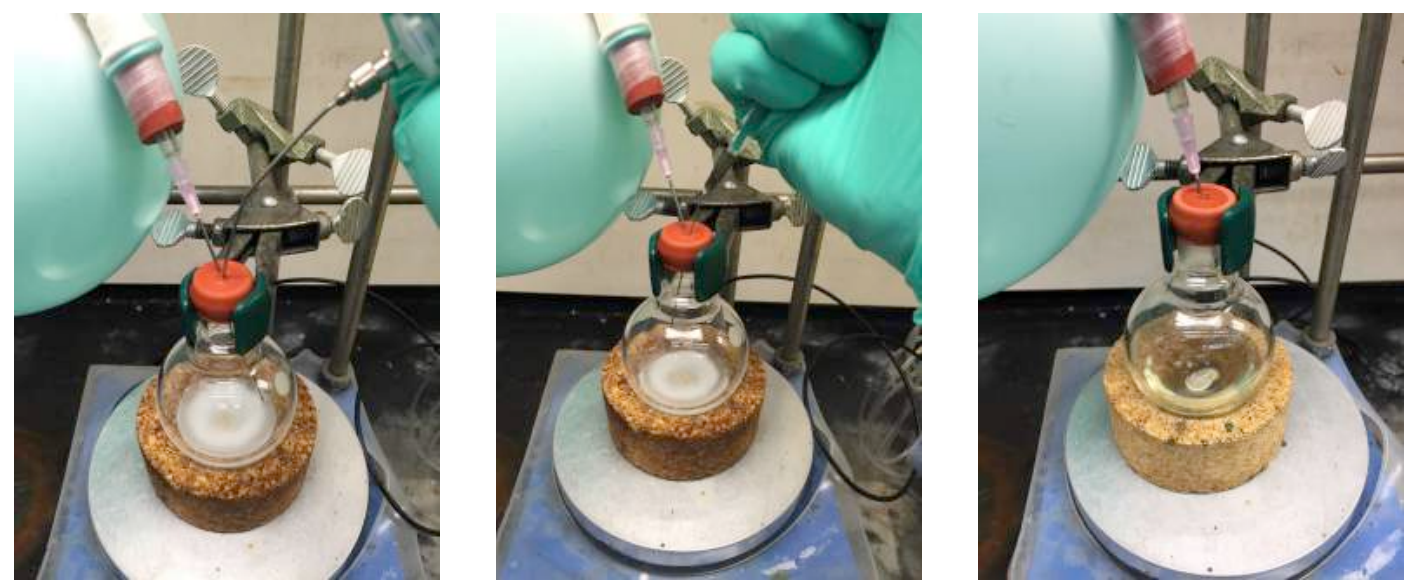

Left: addition of THF. Center: addition of triethylamine. Right: activation after 2 hours at rt. 

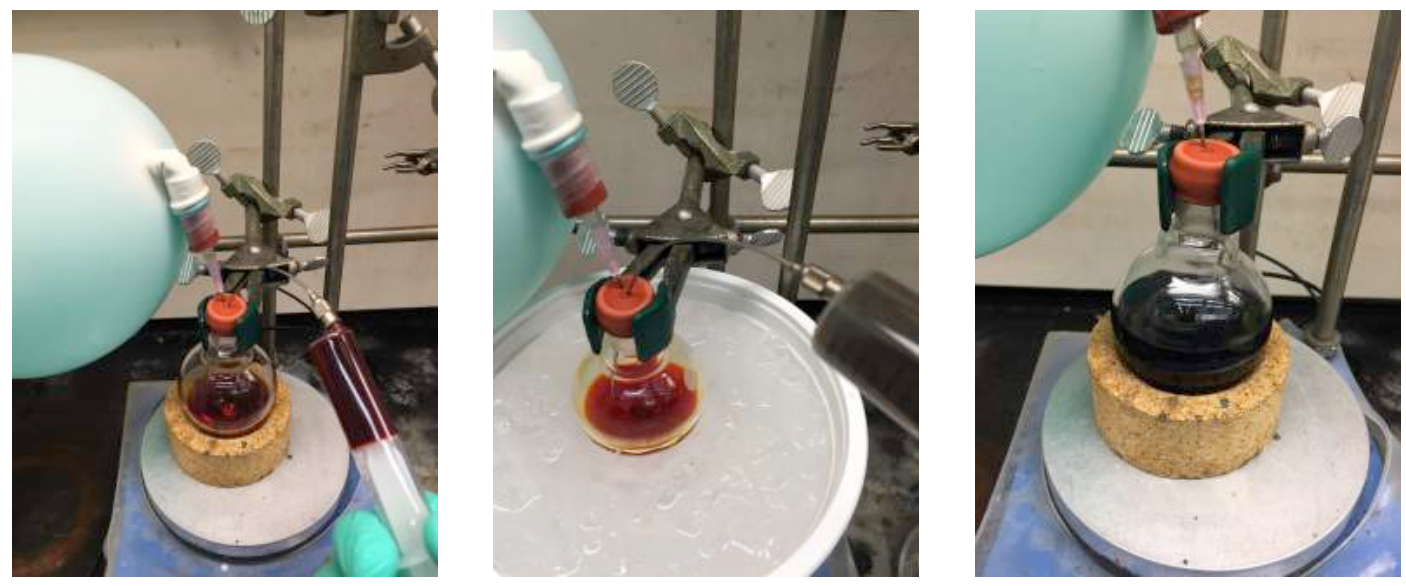

Left: addition of $\mathrm{Fe} /$ ligand solution. Center: addition of the organozinc at $0{ }^{\circ} \mathrm{C}$. Right: reaction after $1 \mathrm{~h}$ at r.t.
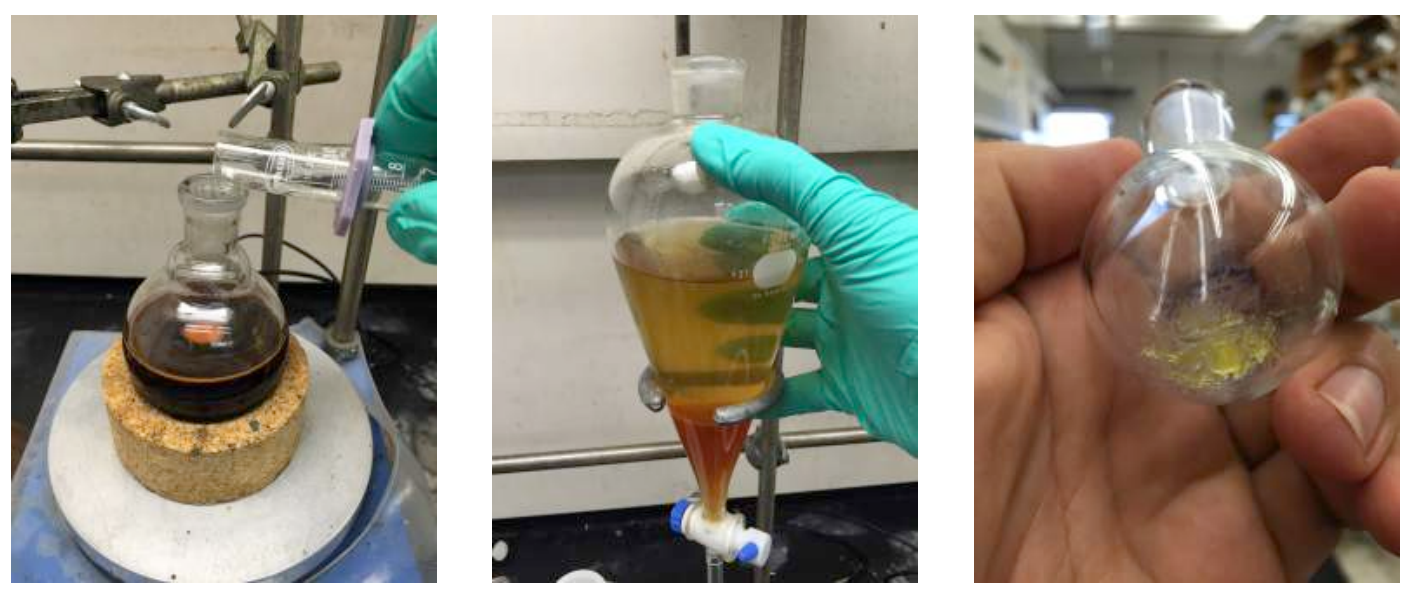

Left: quench of the reaction with $1 \mathrm{M} \mathrm{HCl}$. Center: dilution and extraction of the organic layer.

Right: pure product after column chromatography.

\section{Step 2: hydrolysis.}
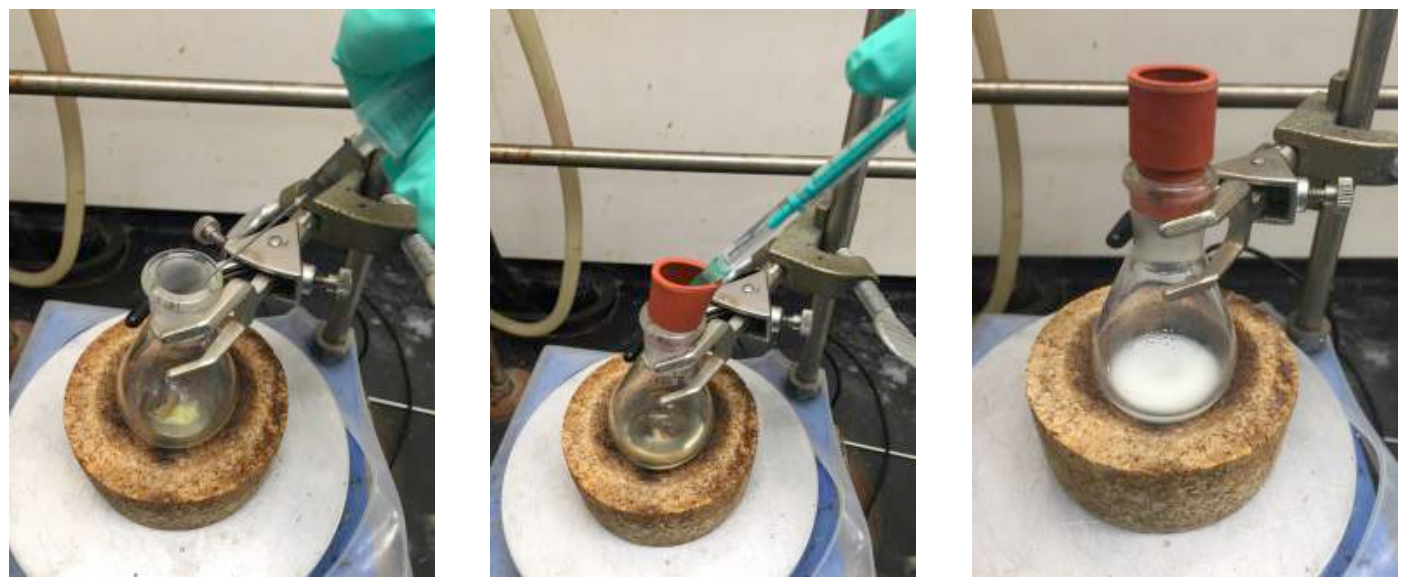

Left: THF is added to the ester in an open flask. Center: addition of $\mathrm{NaOH}$ in $\mathrm{MeOH}$. Right: after stirring at r.t. for $16 \mathrm{~h}$. 

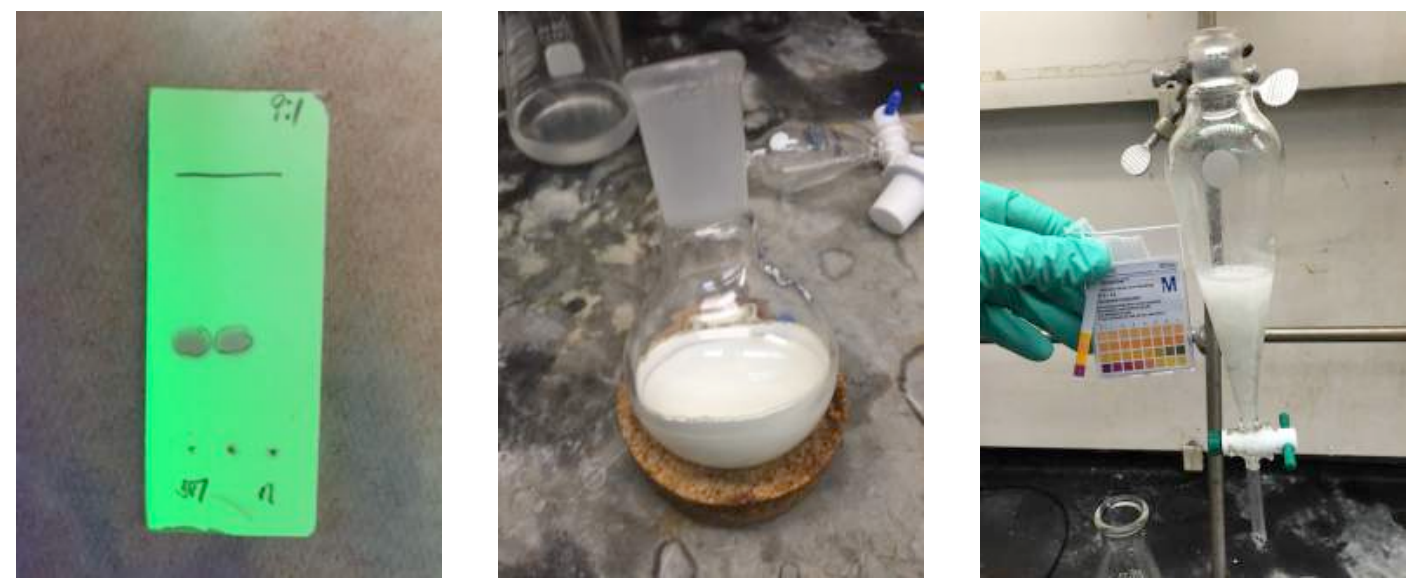

Left: TLC plate of the reaction mixture (left: starting material; center: cross-spot; right: reaction mixture). Center: evaporation to dryness. Right. $\mathrm{pH}$ adjusted with $1 \mathrm{M} \mathrm{HCl}$ in a separating funnel after dissolving with $\mathrm{CH}_{2} \mathrm{Cl}_{2}$.
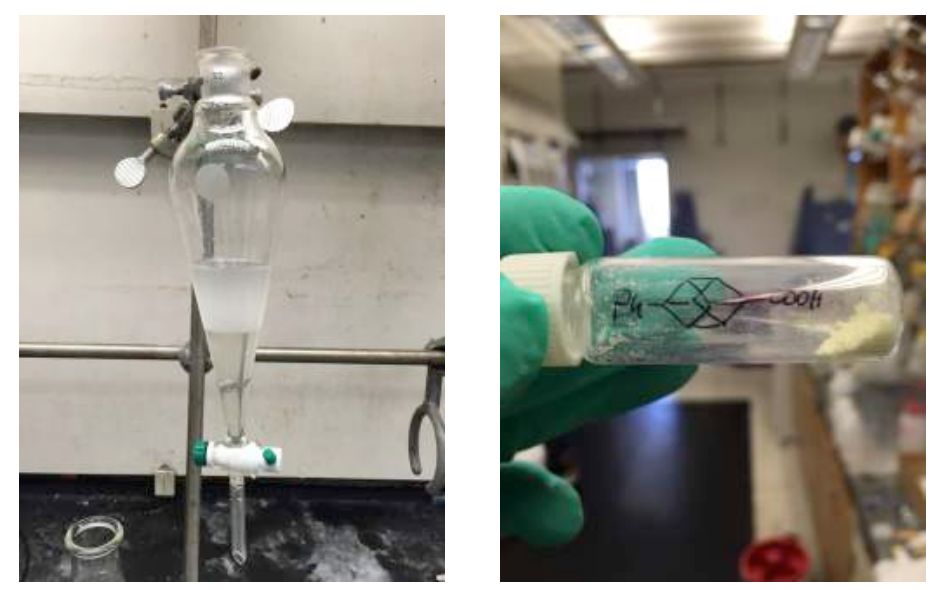

Left: two phases. Right: pure product after evaporation of the organic layer.

\section{Step 3. Second arylation}
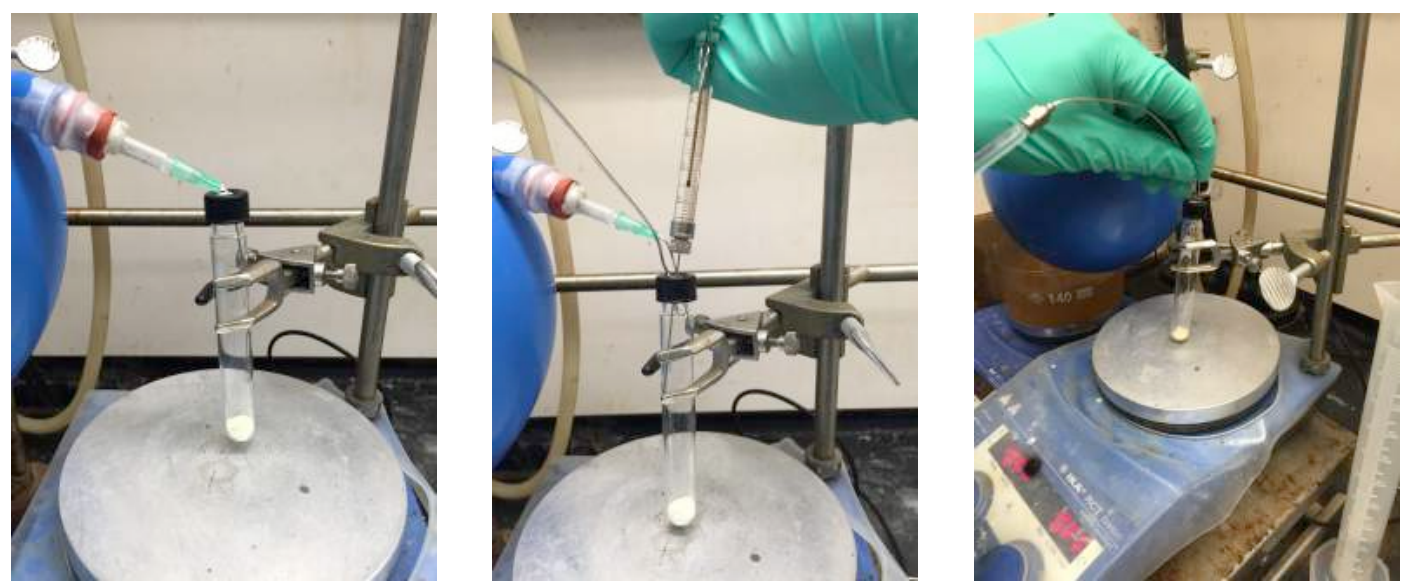

Left: starting carboxylic acid and HATU in aculture tube under Ar. Center: addition of triethylamine. Right: addition of THF. 

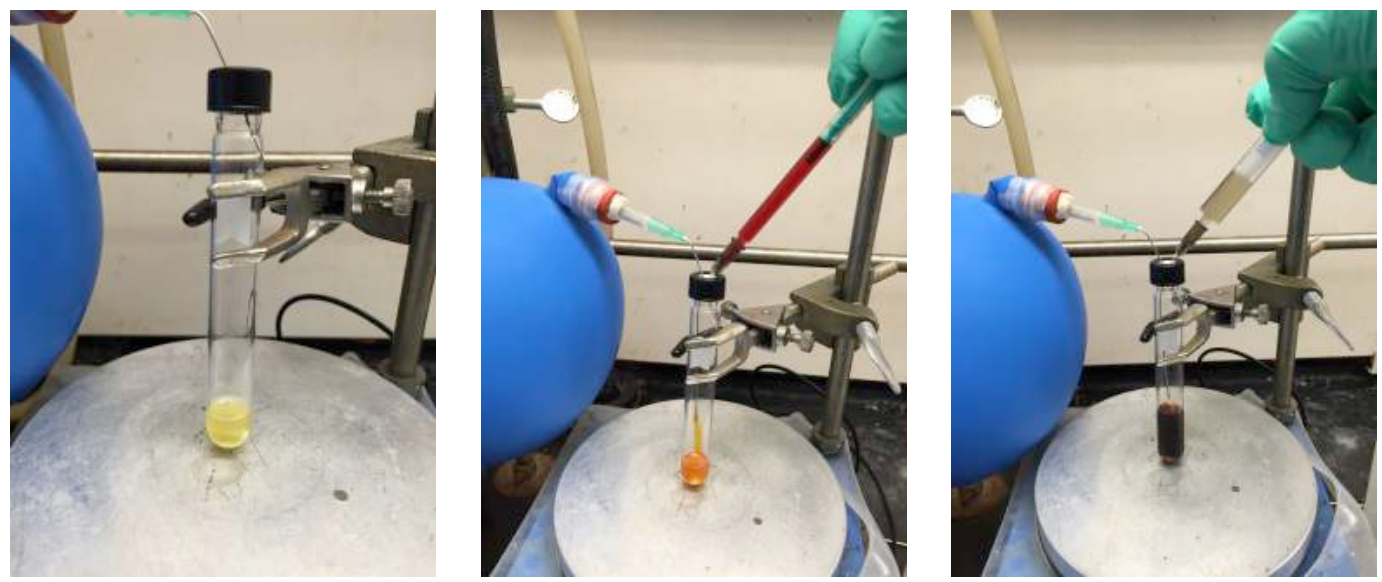

Left: reaction tube after $2 \mathrm{~h}$ activation at r.t. Center: addition of Fe/ligand solution. Right: addition of the organozinc reagent at r.t. in one portion.
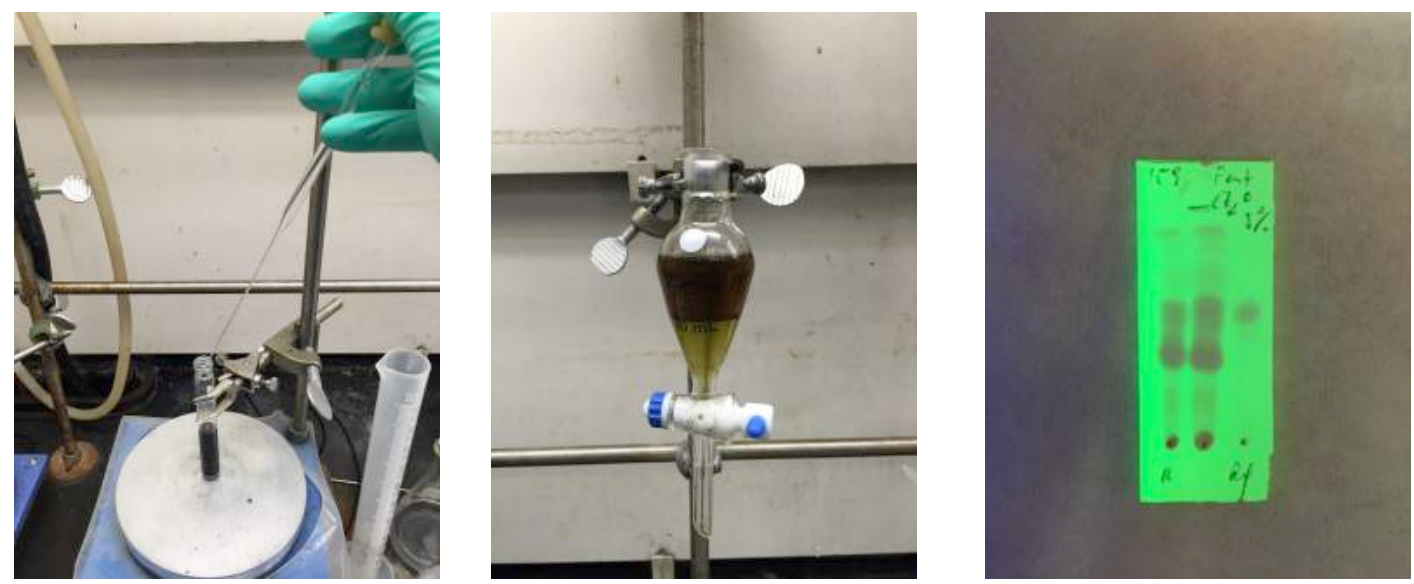

Left: quench of the reaction mixture after $1 \mathrm{~h}$ with $1 \mathrm{~N} \mathrm{HCl}$. Center: extraction after dilution with diethyl ether. Right: TLC plate of the crude (left: reaction mixture; center: cross-spot; right: pure product).

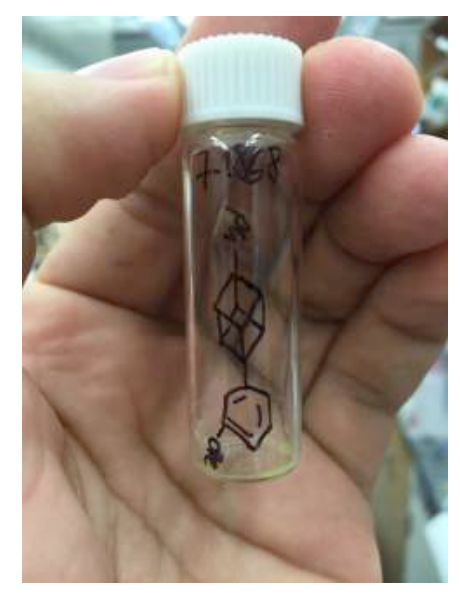

Final product after PTLC. 
17. NMR spectra
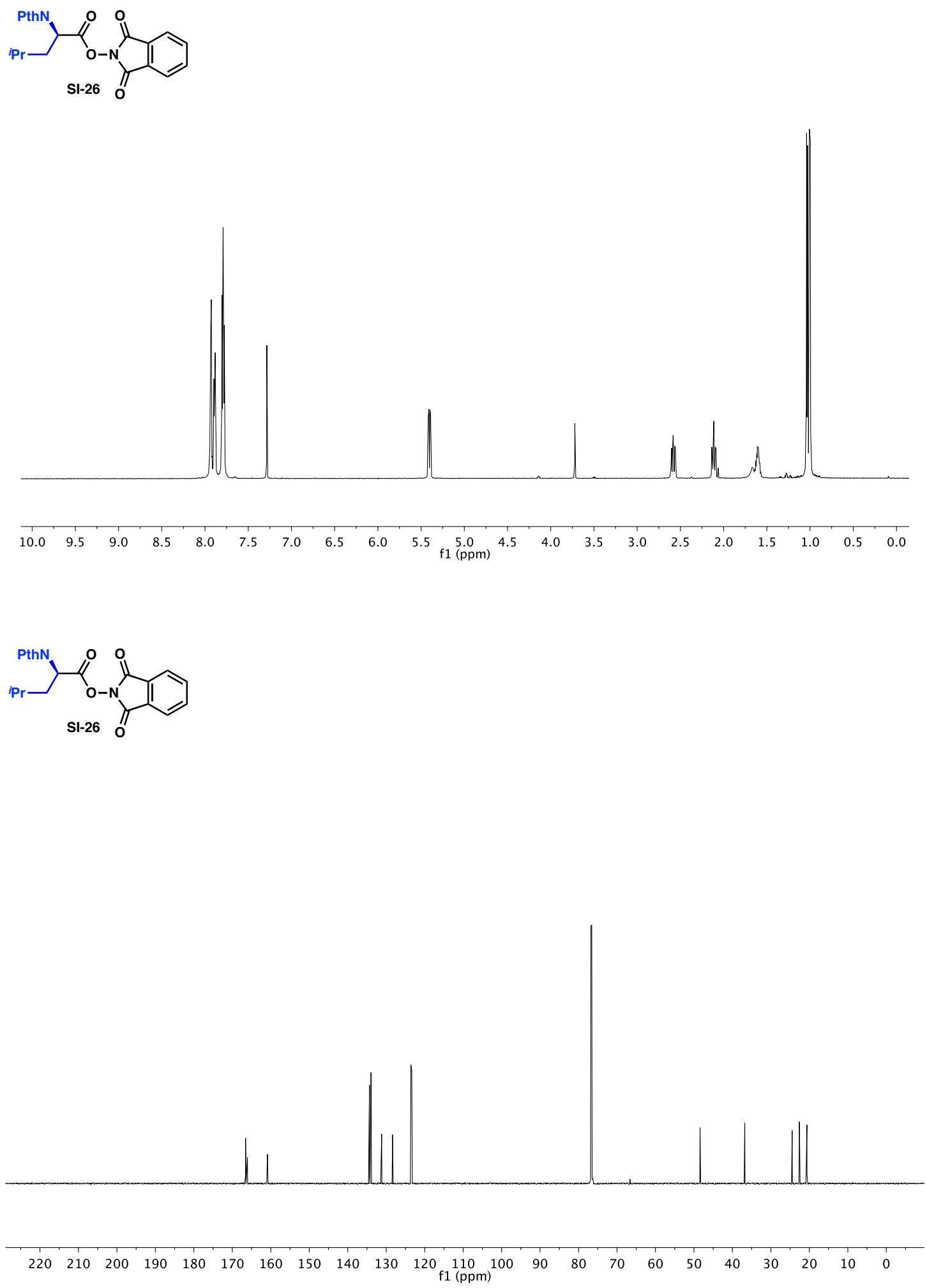

S87 

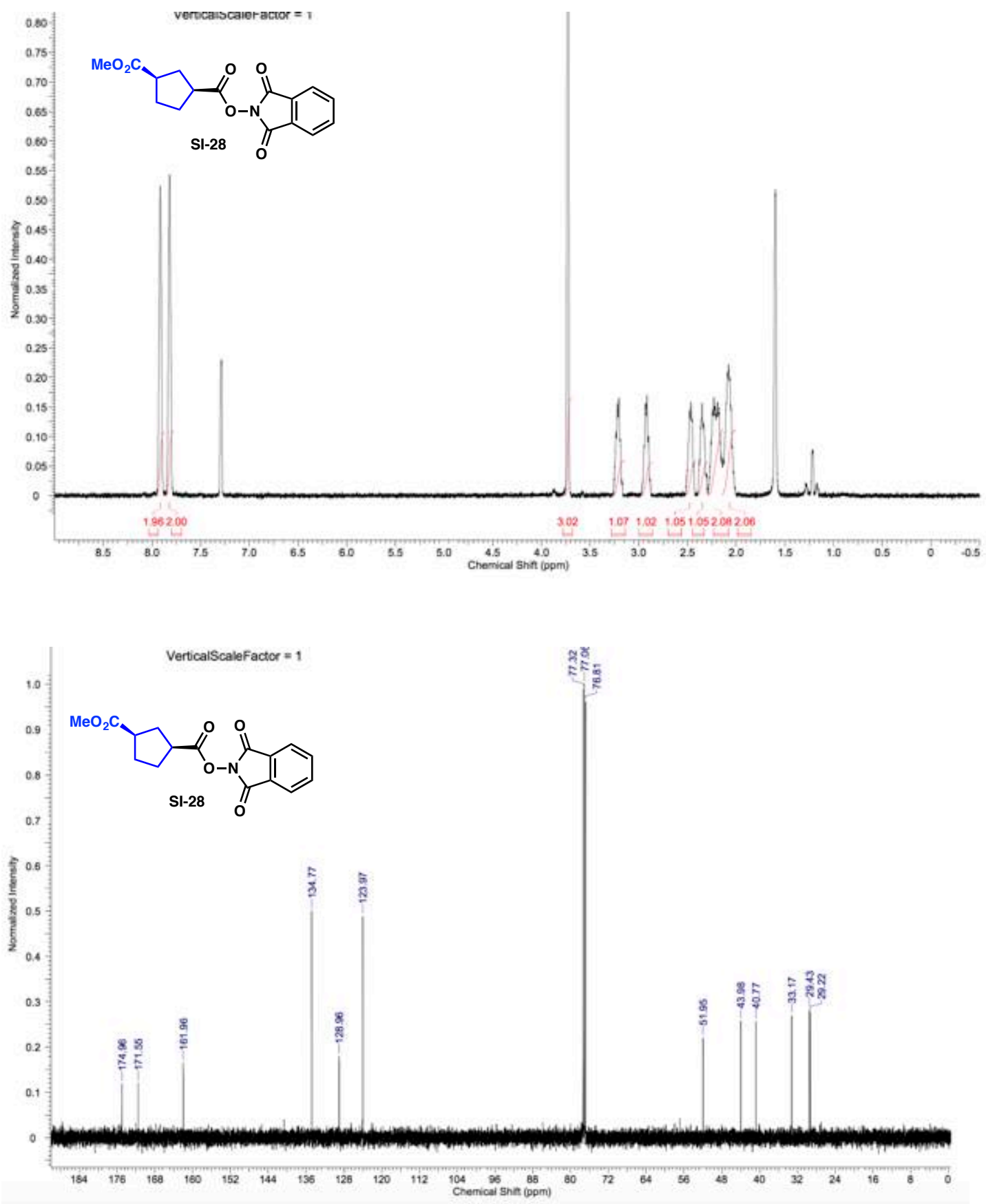

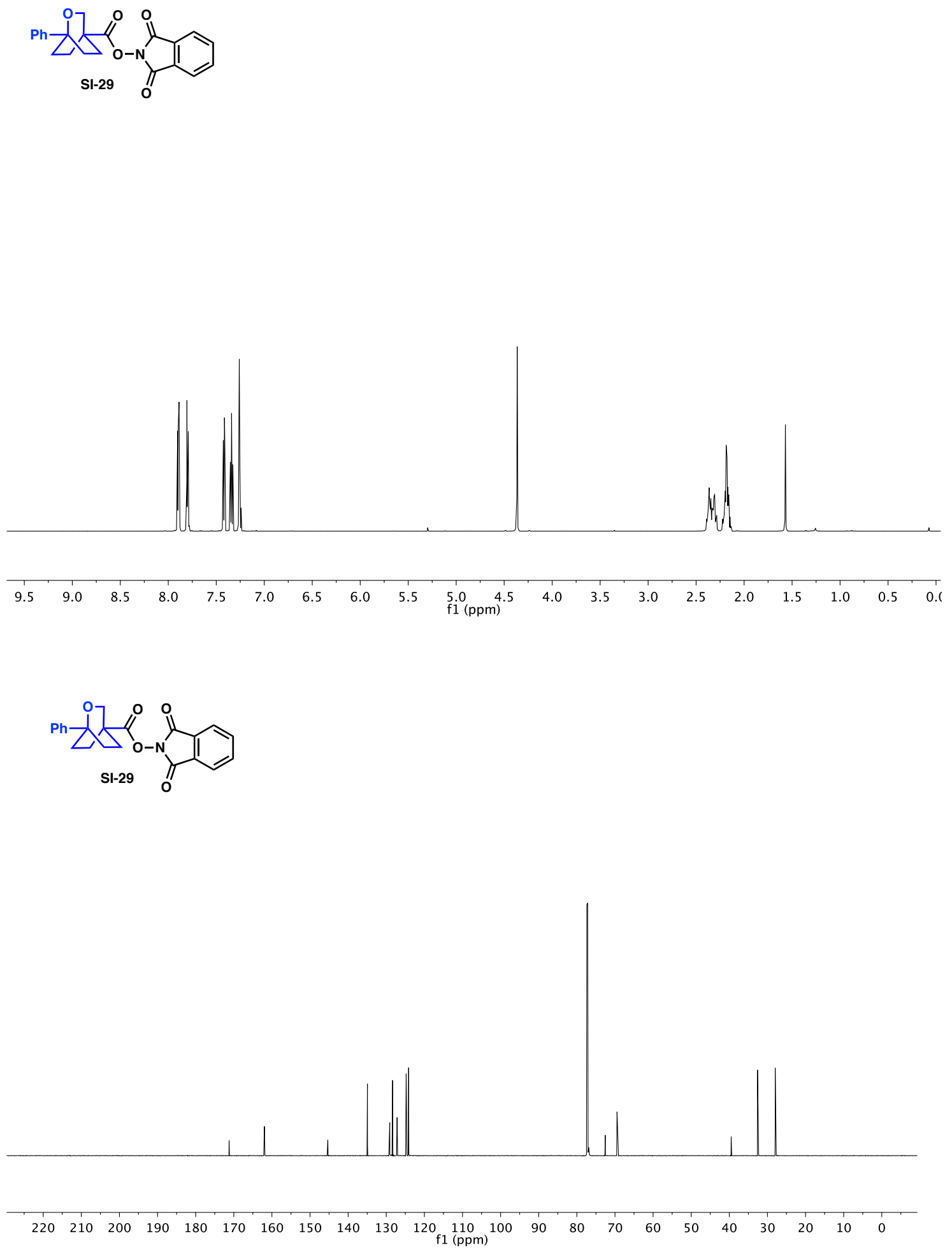

S89 

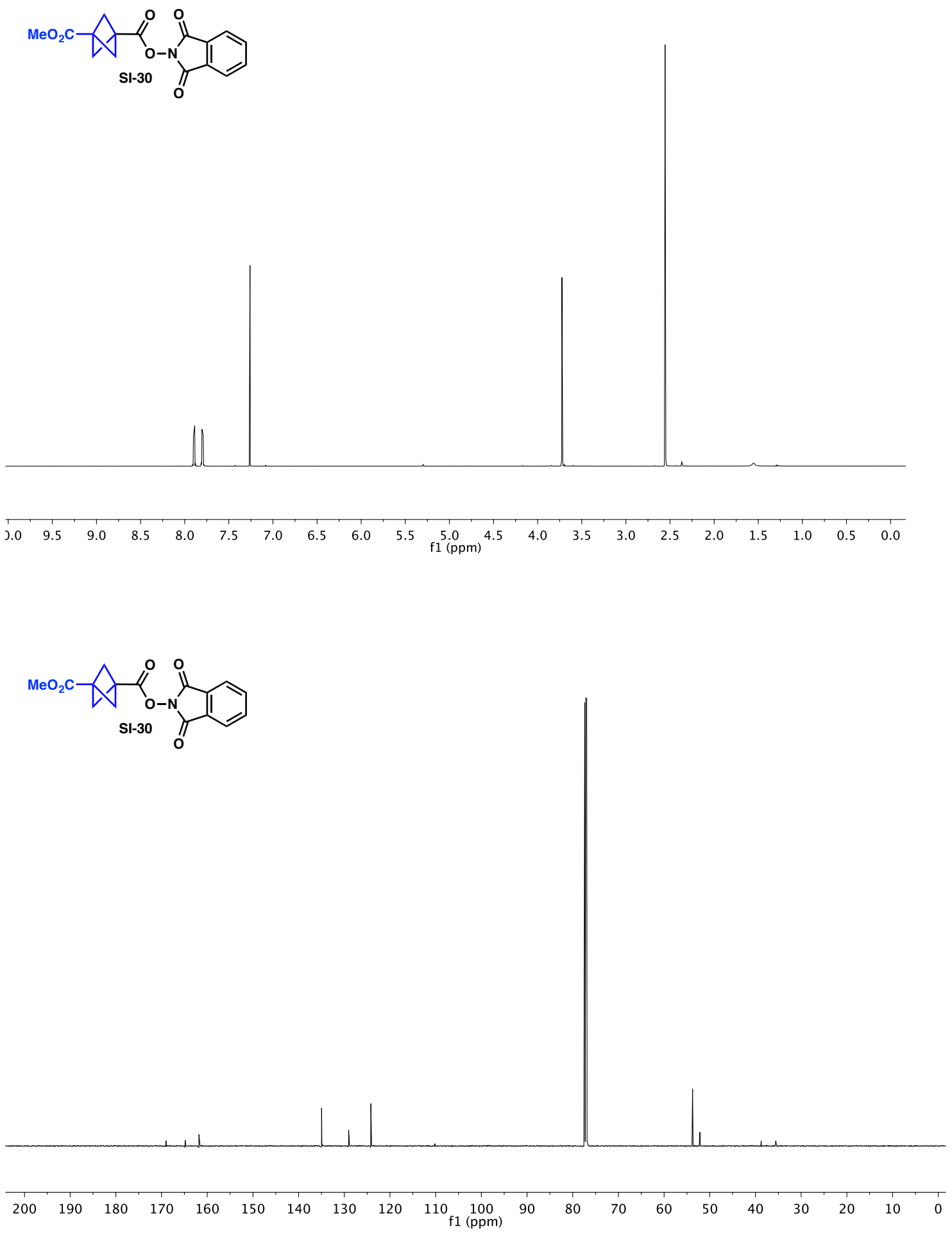

S90 

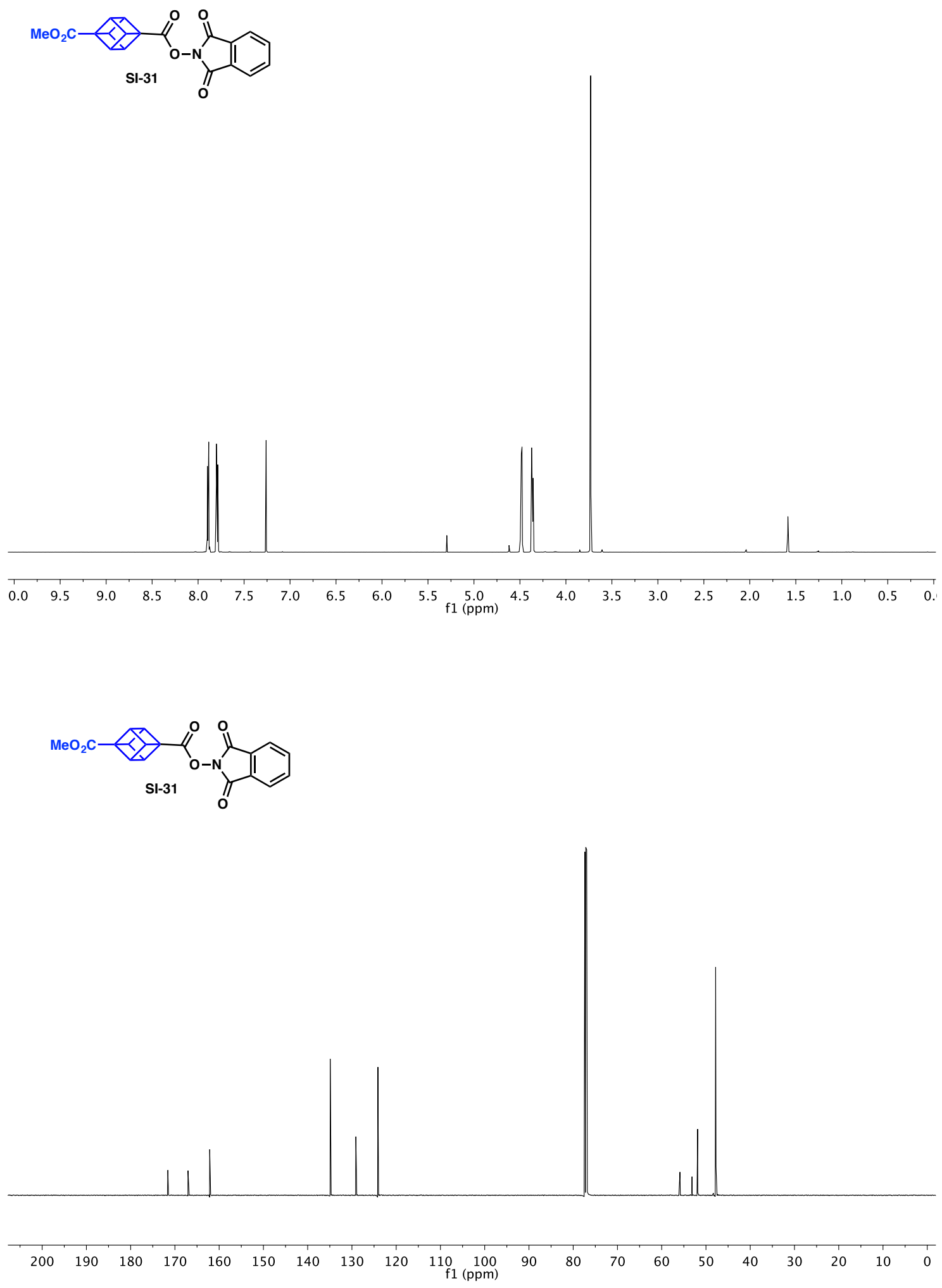

S91 

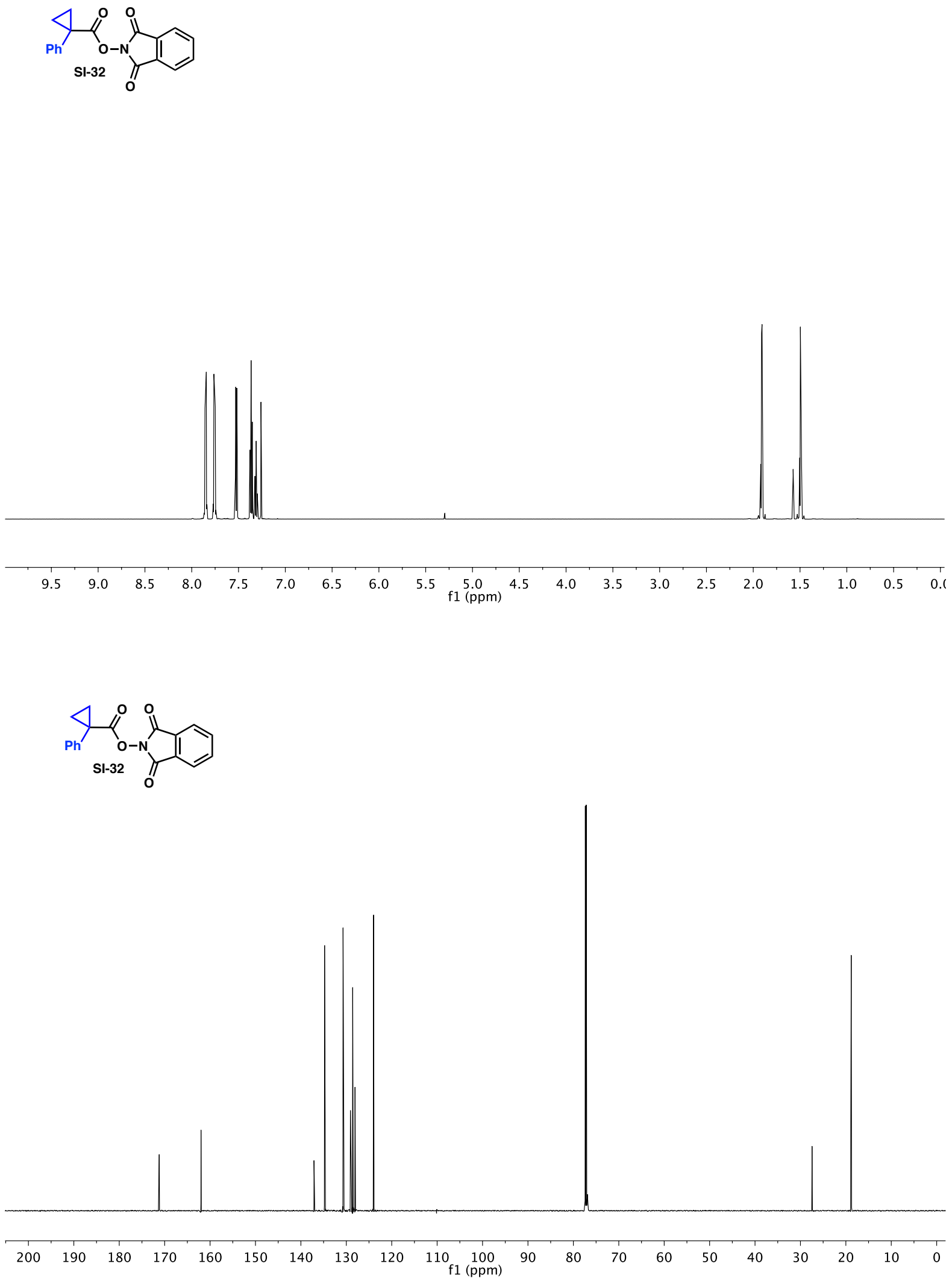

S92 

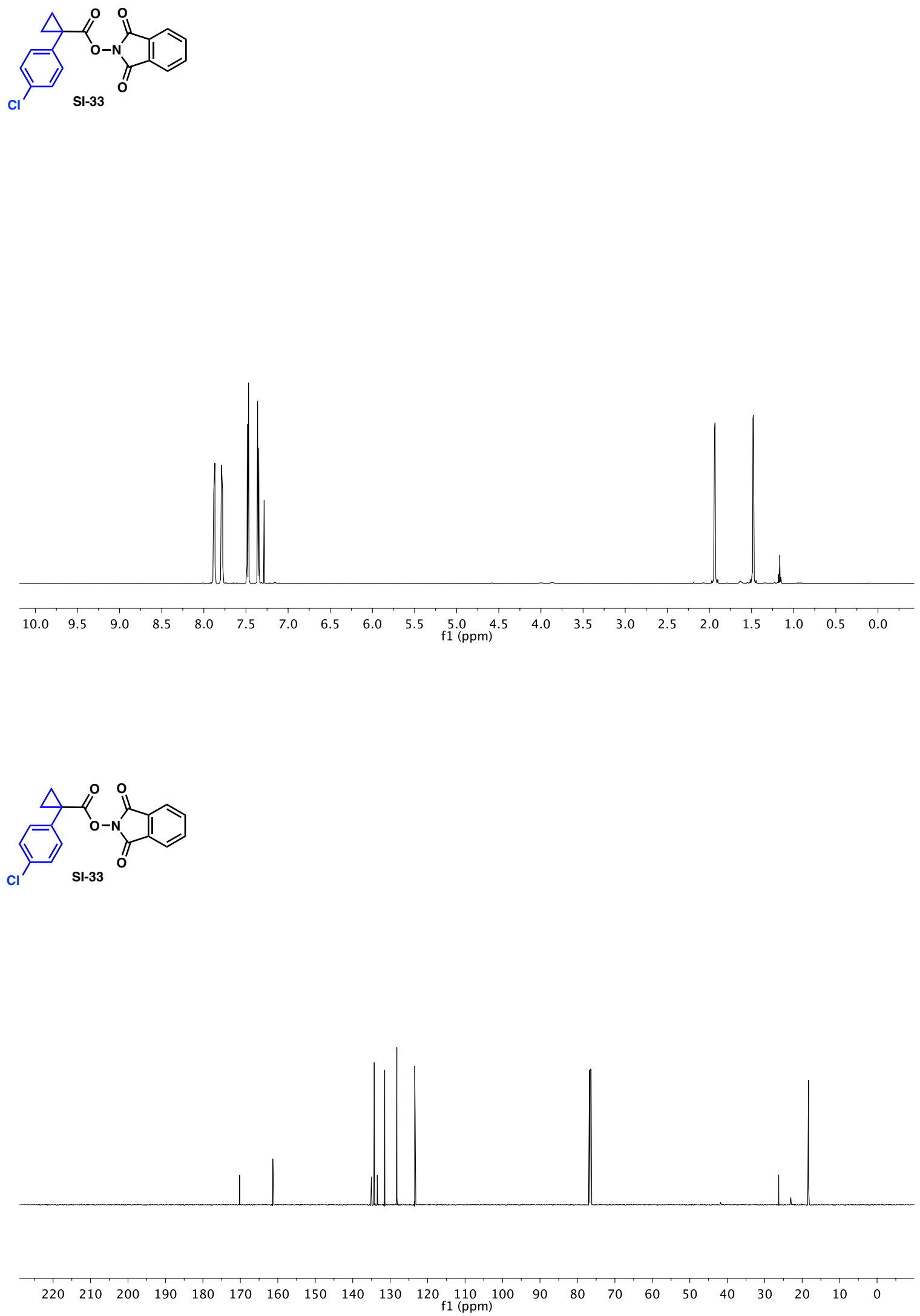

S93 

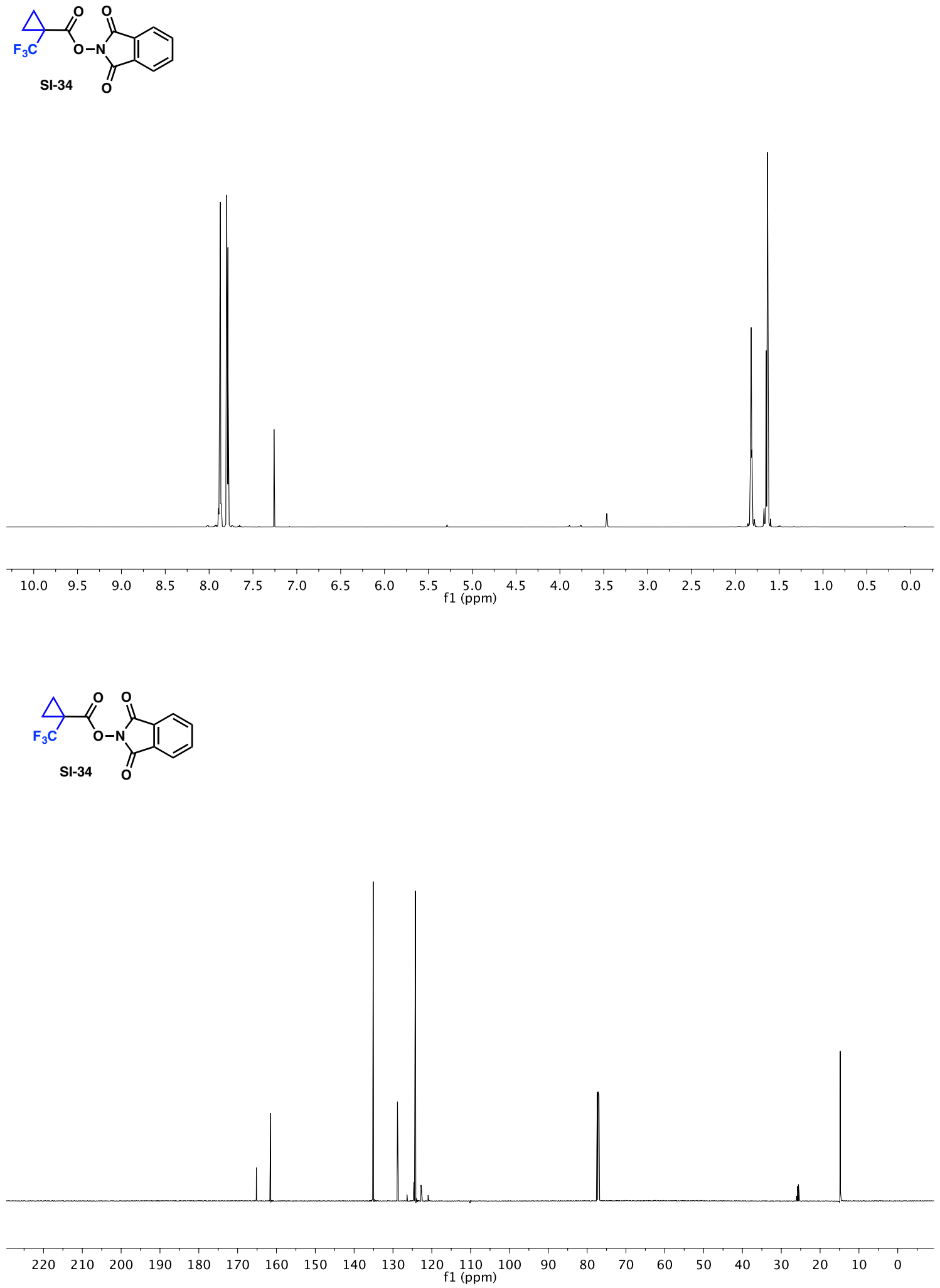

S94 

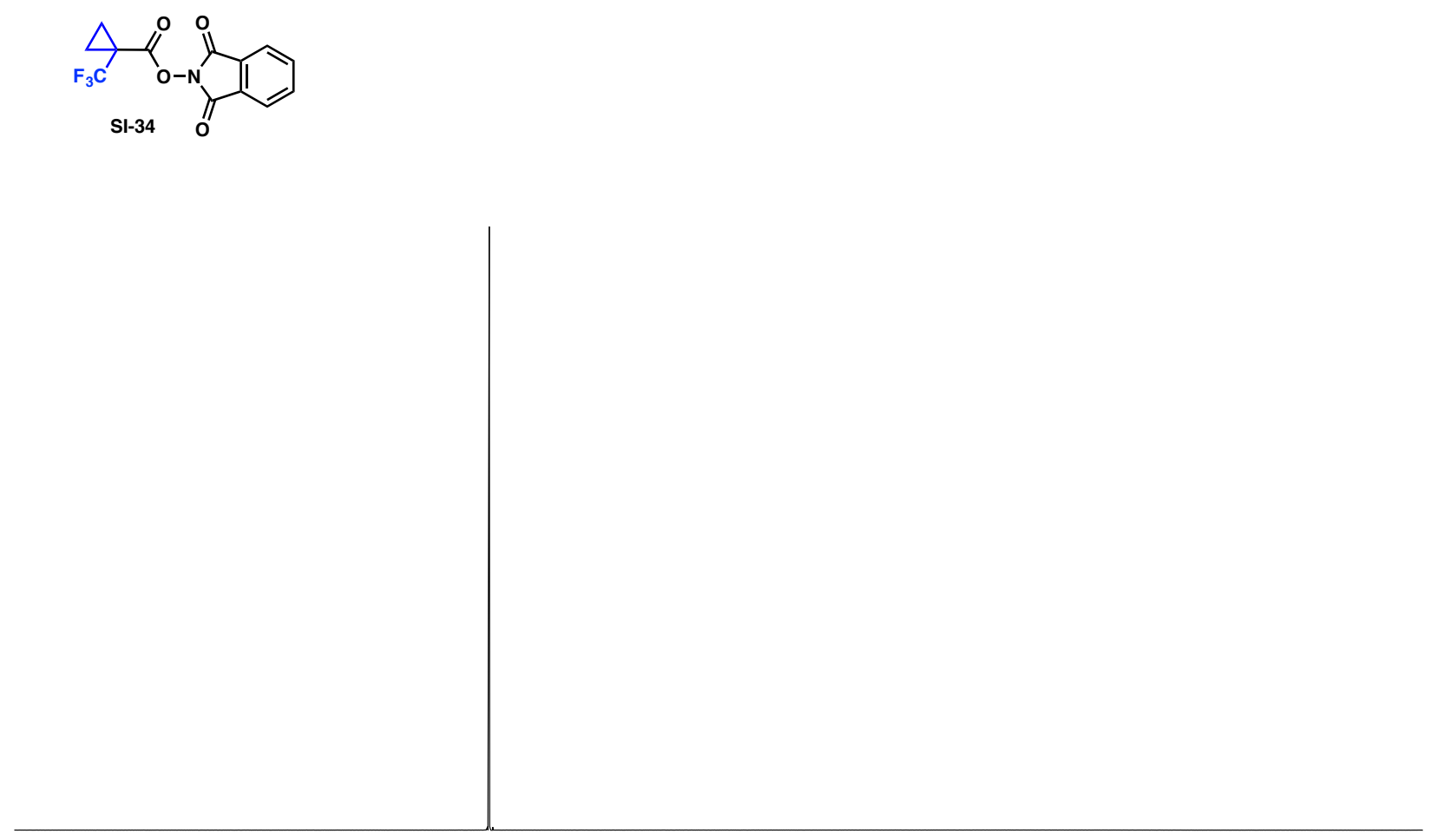

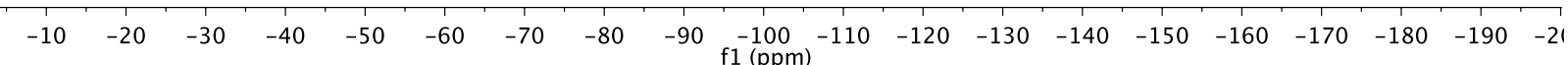



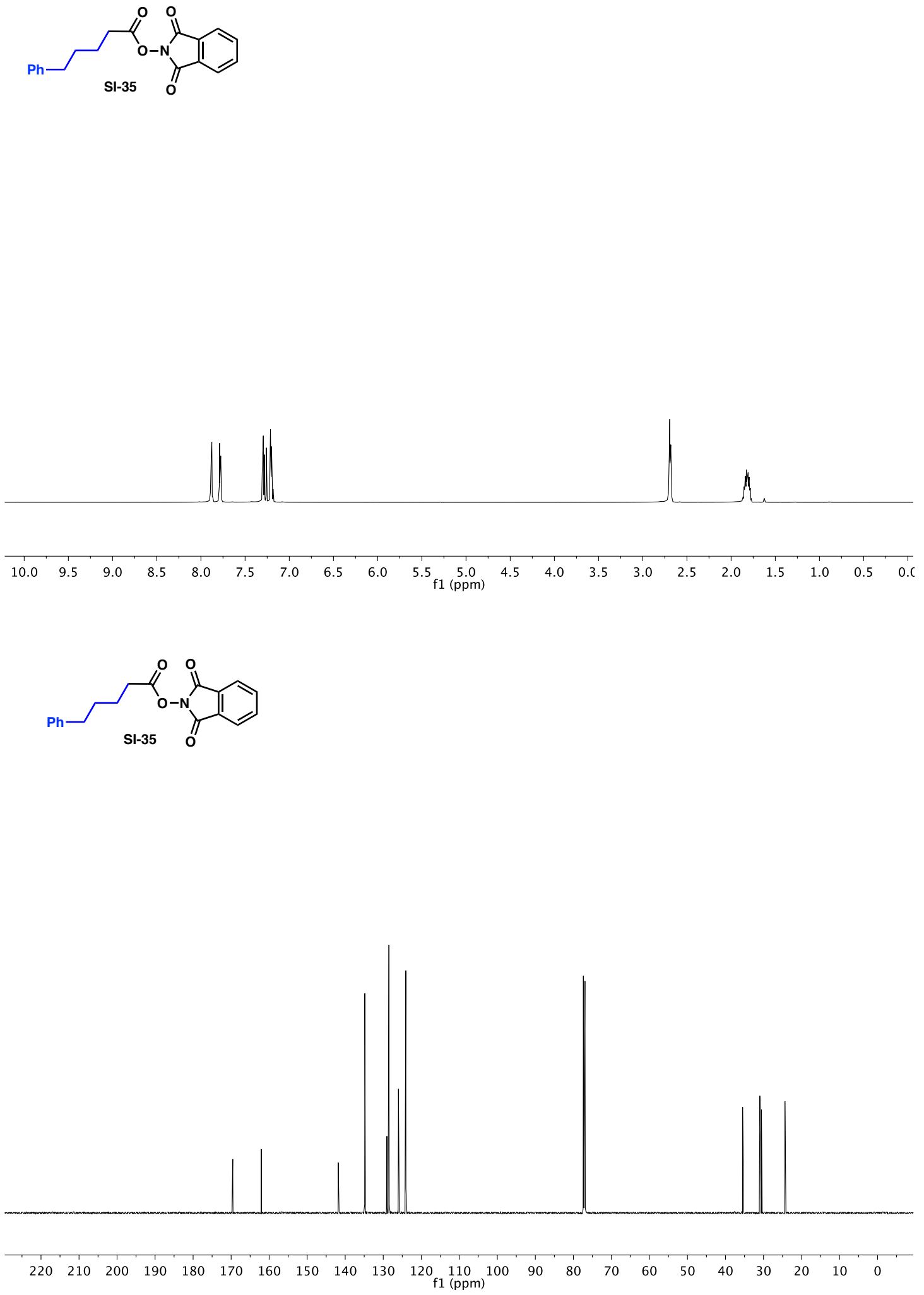

S96 

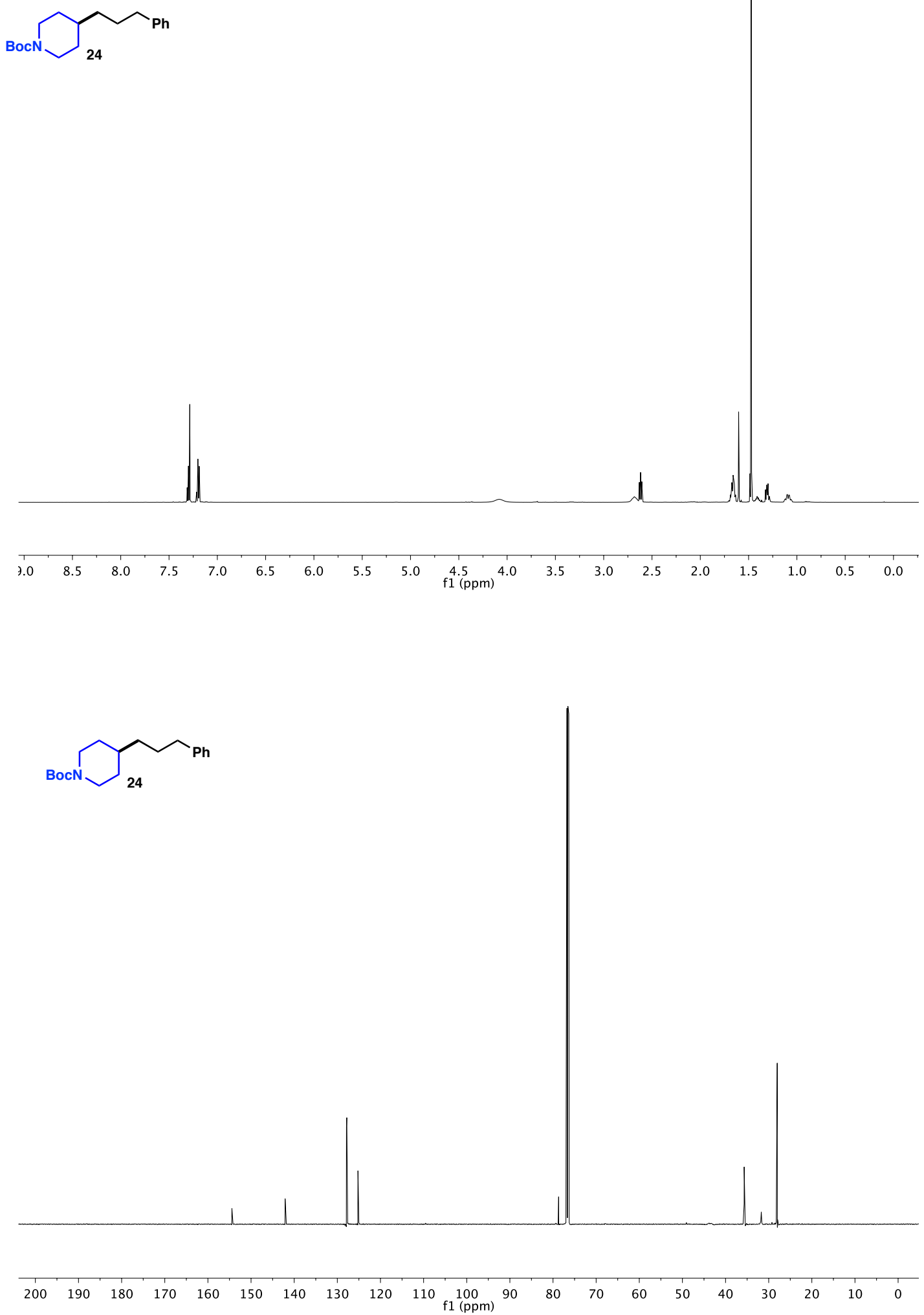

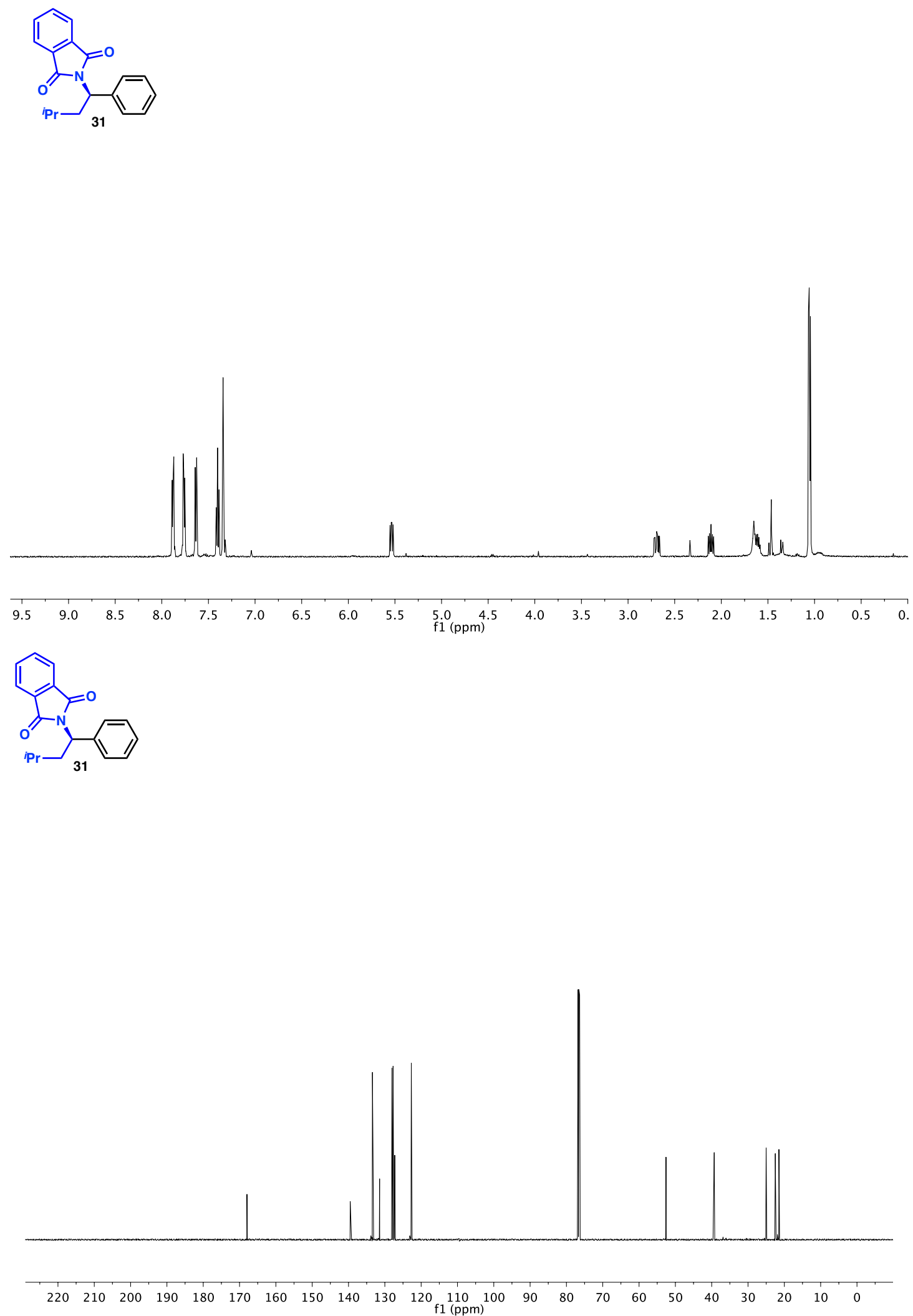


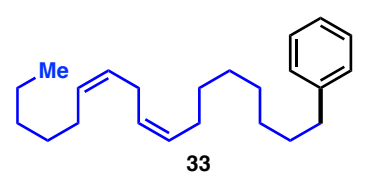

33

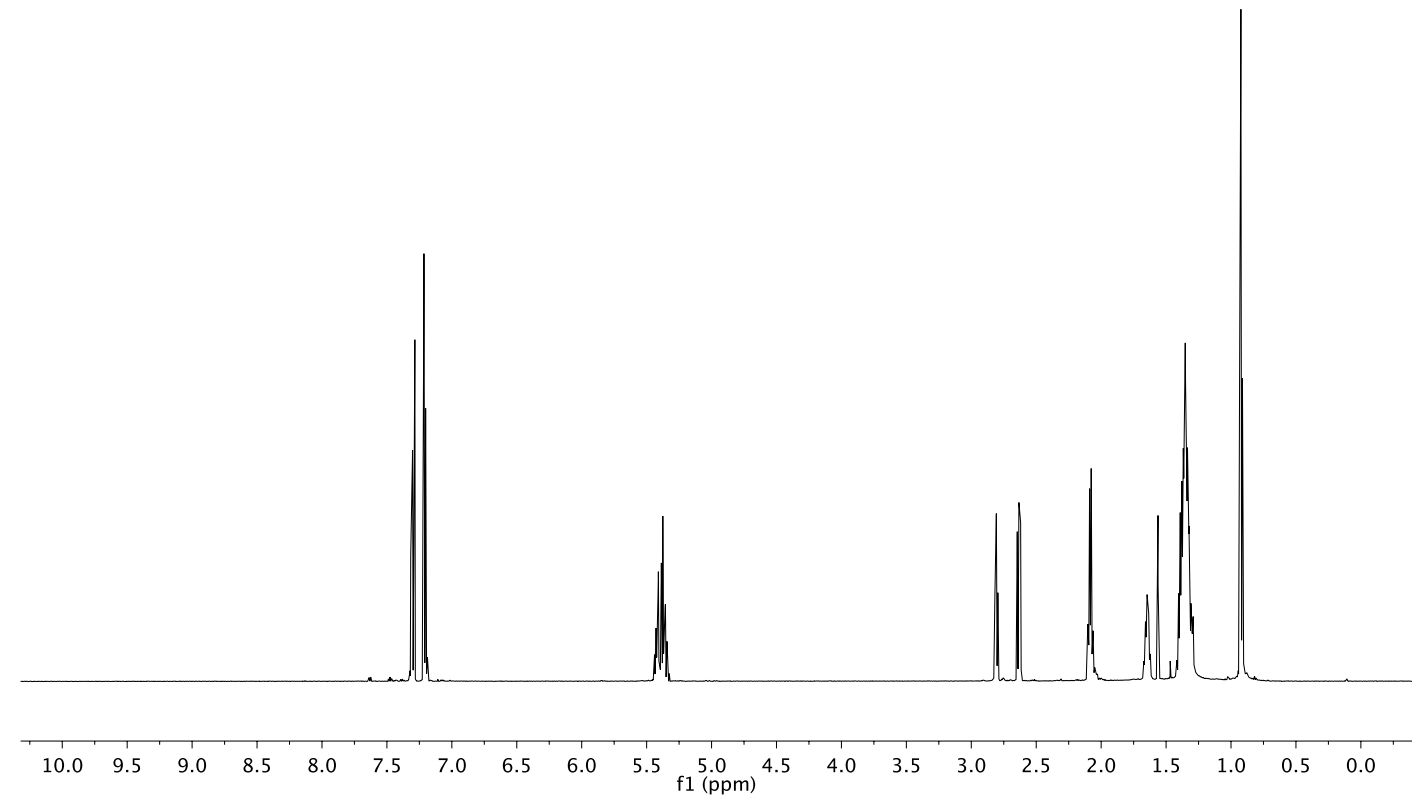

$P_{33}^{M e}$

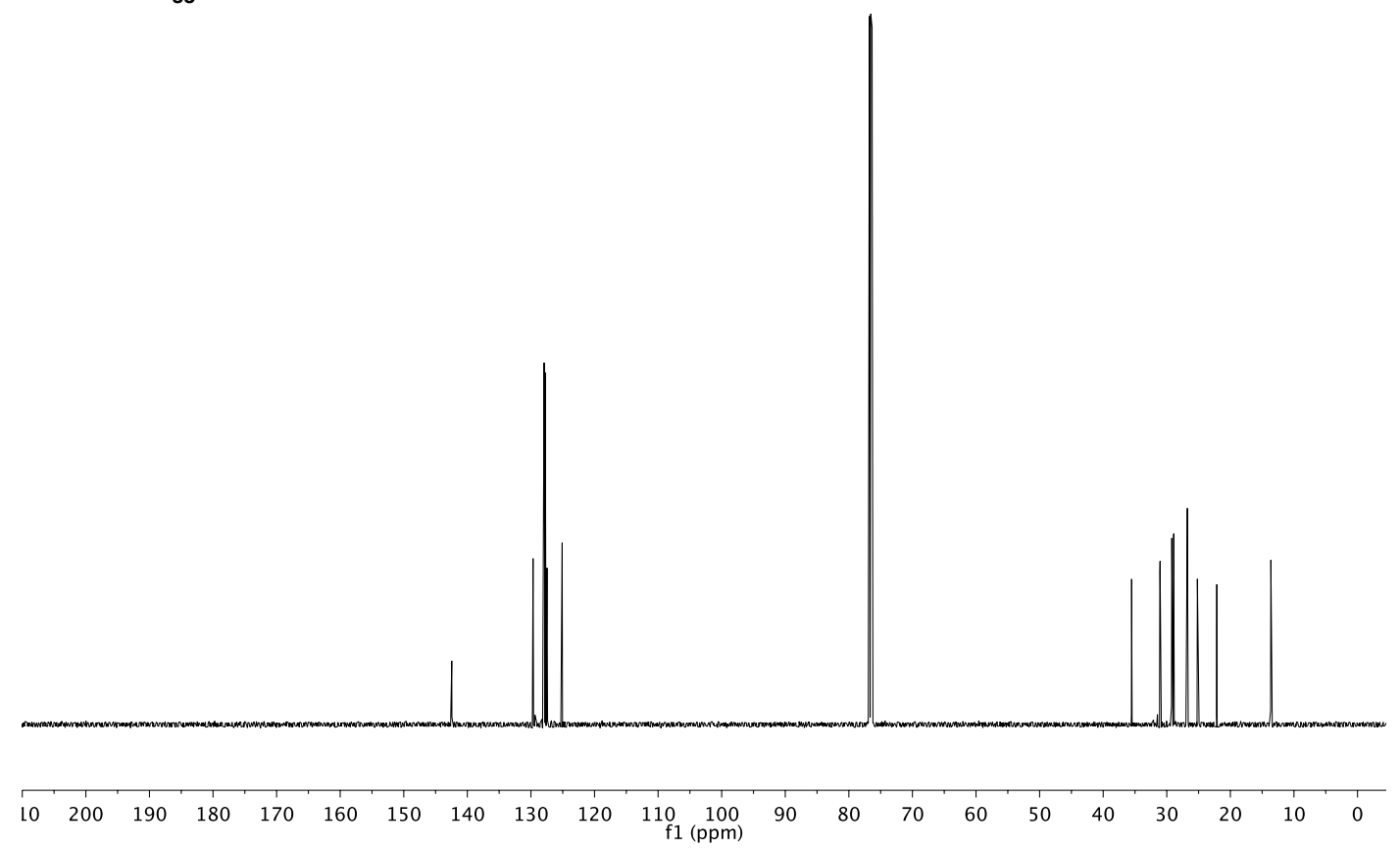




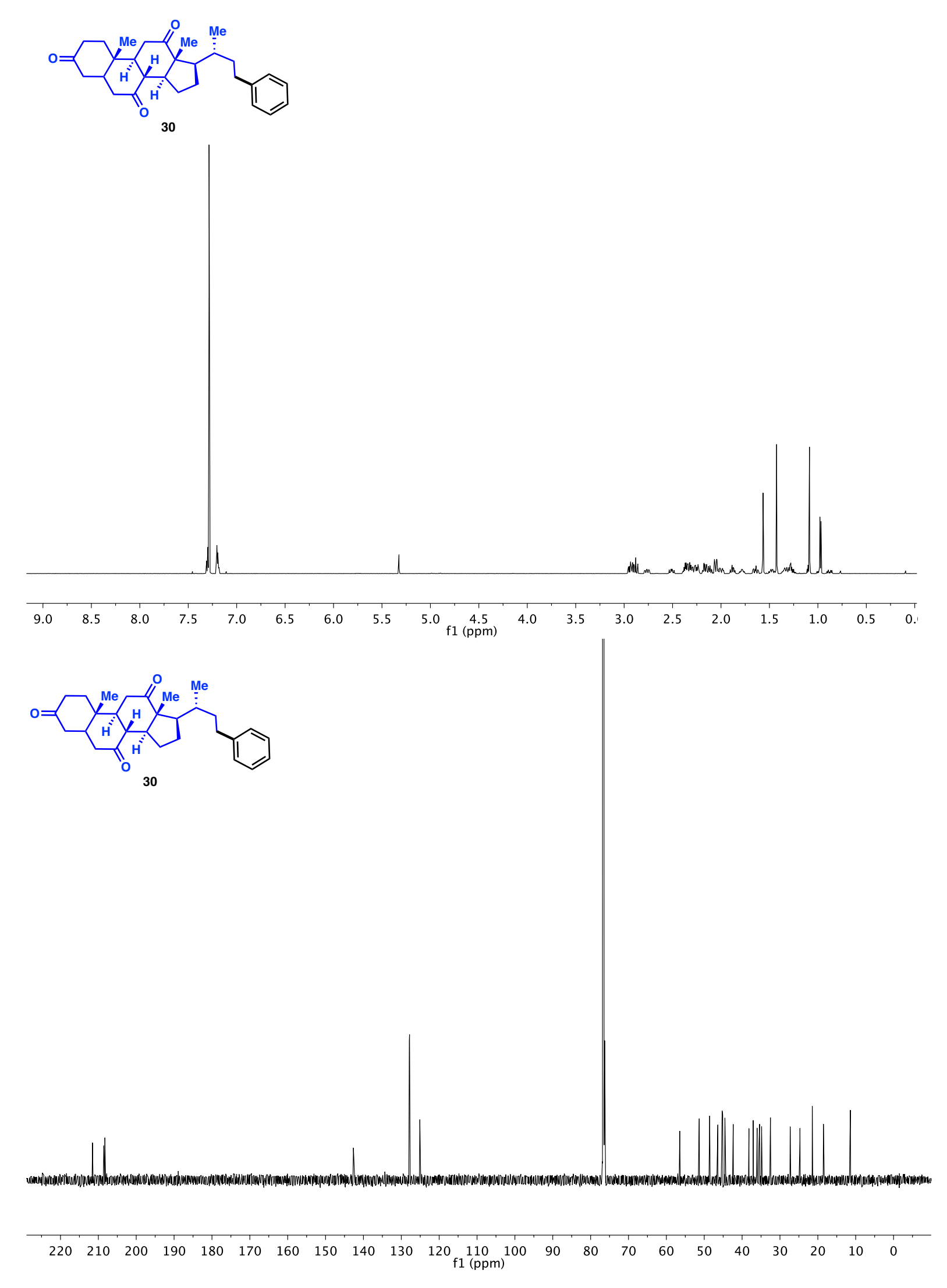

S100 

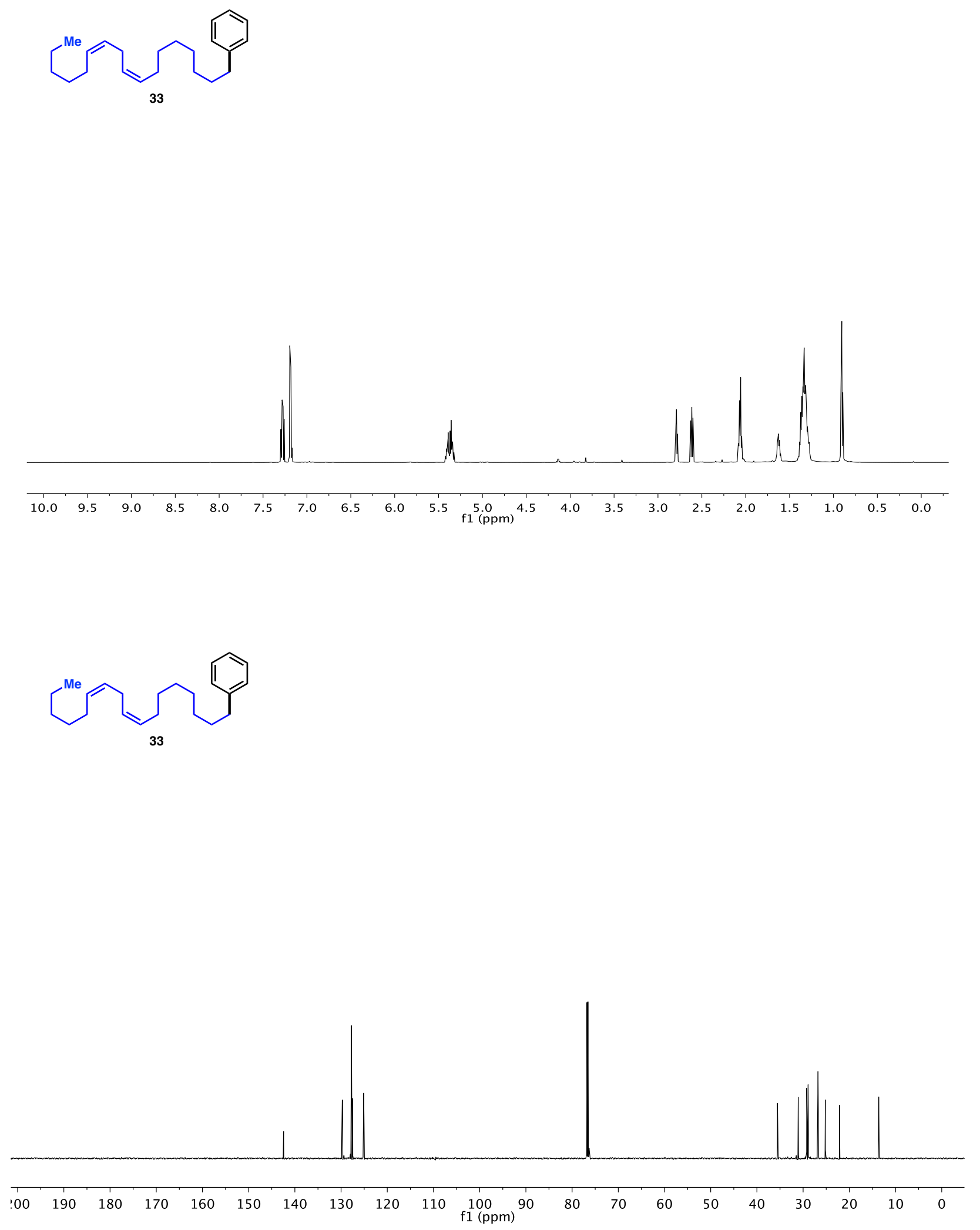


\section{Q}
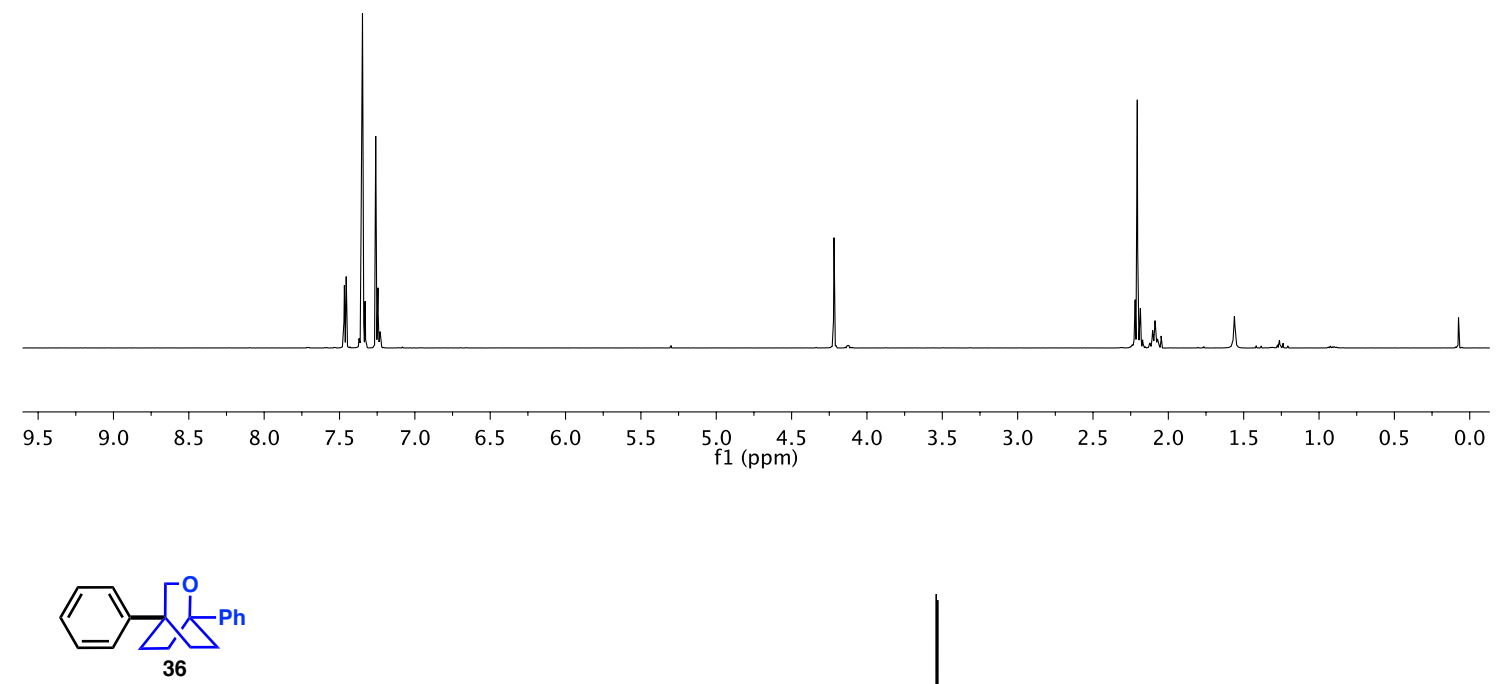

36

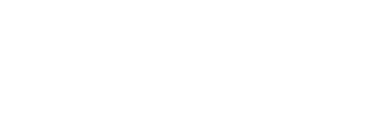



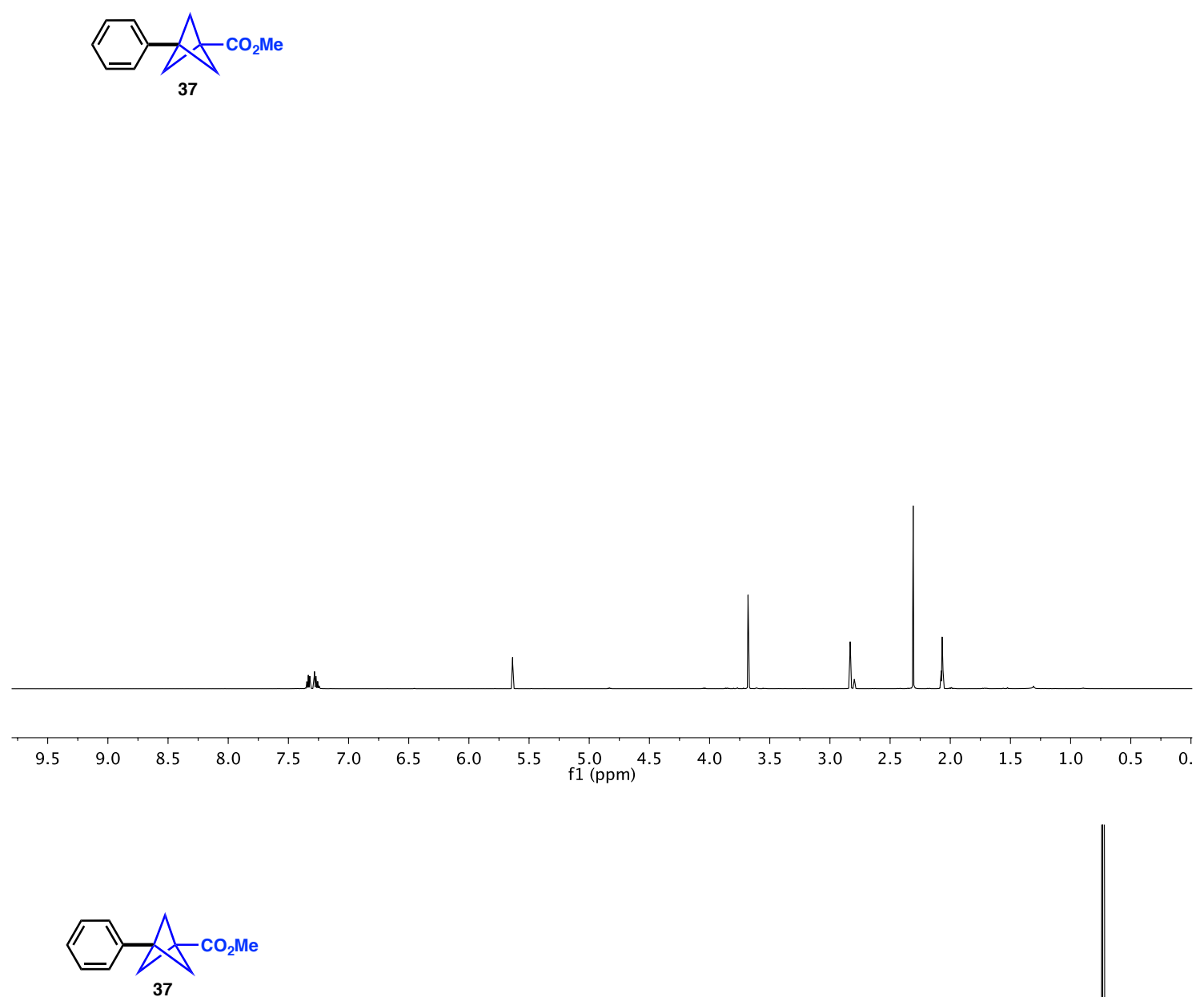

(ppm)

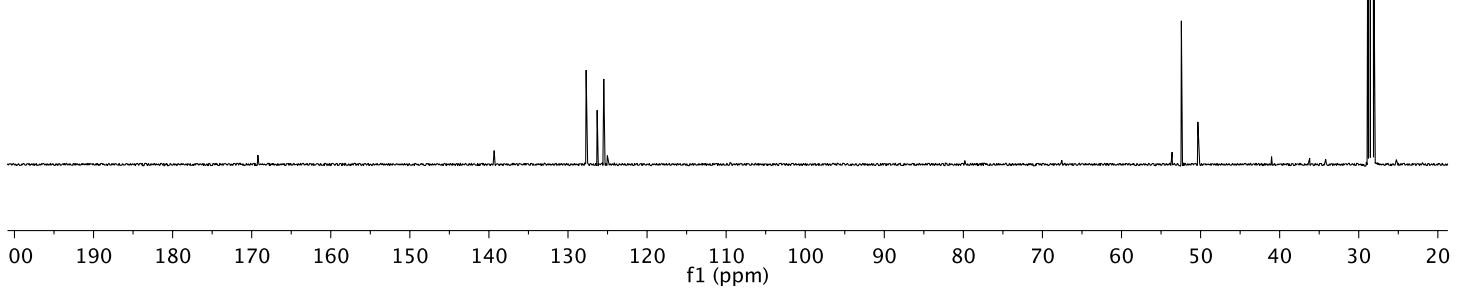



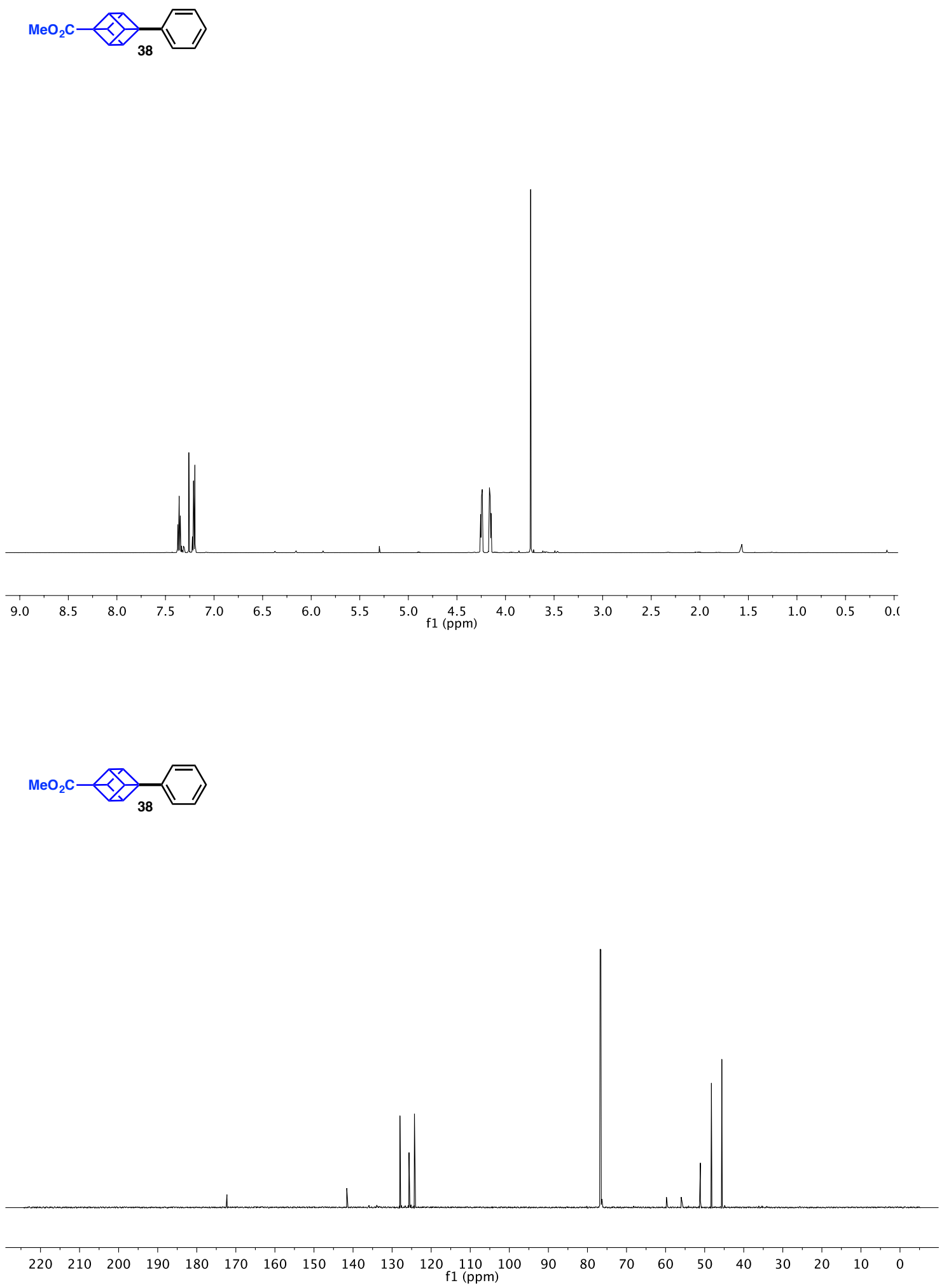

S104 
<smiles>c1ccc(C2(c3ccccc3)CC2)cc1</smiles>

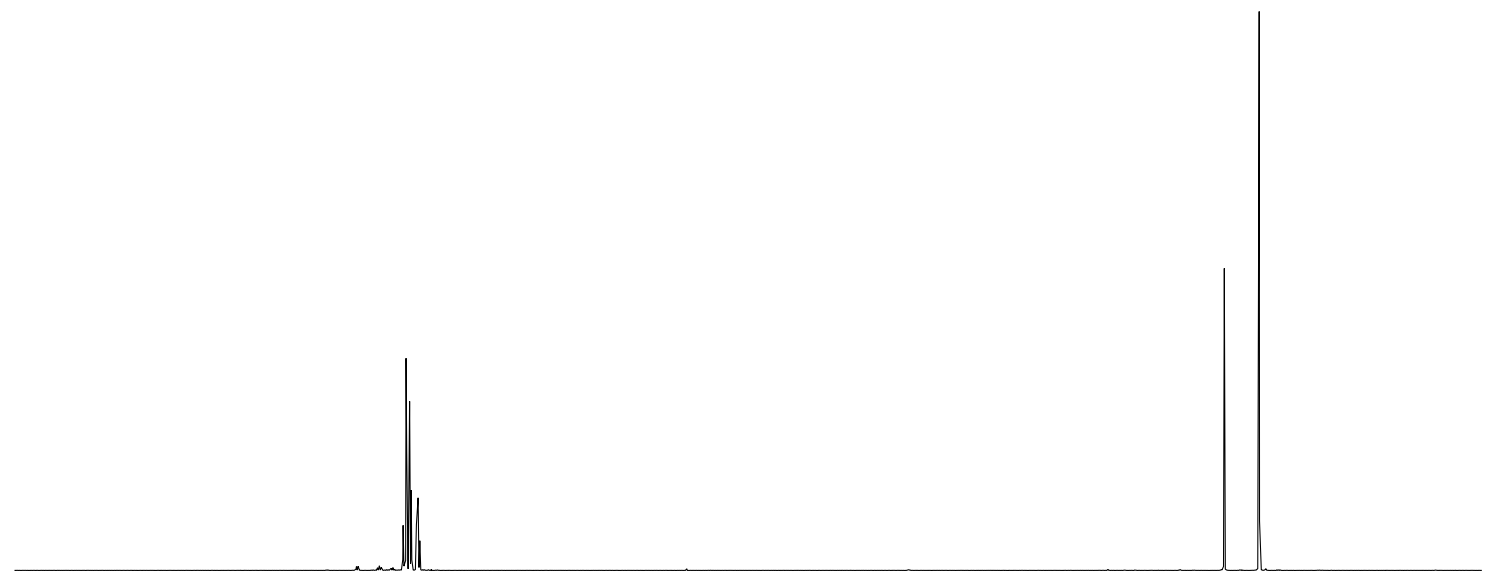

$\begin{array}{lllllllllllllllllllll}9.5 & 9.0 & 8.5 & 8.0 & 7.5 & 7.0 & 6.5 & 6.0 & 5.5 & \begin{array}{c}5.0 \\ \mathrm{f}(\mathrm{ppm})\end{array} & 4.5 & 4.0 & 3.5 & 3.0 & 2.5 & 2.0 & 1.5 & 1.0 & 0.5 & 0.0\end{array}$<smiles>c1ccc(C2(c3ccccc3)CC2)cc1</smiles>

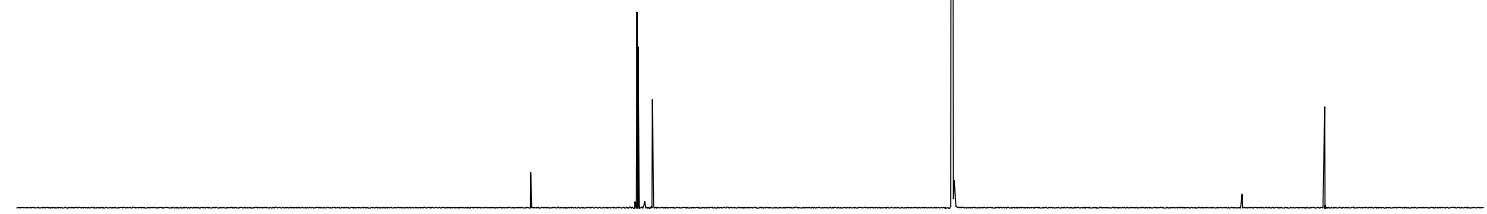

$\begin{array}{lllllllllllllllllllllll}220 & 210 & 200 & 190 & 180 & 170 & 160 & 150 & 140 & 130 & 120 & 110 & 100 & 90 & 80 & 70 & 60 & 50 & 40 & 30 & 20 & 10 & 0\end{array}$ 

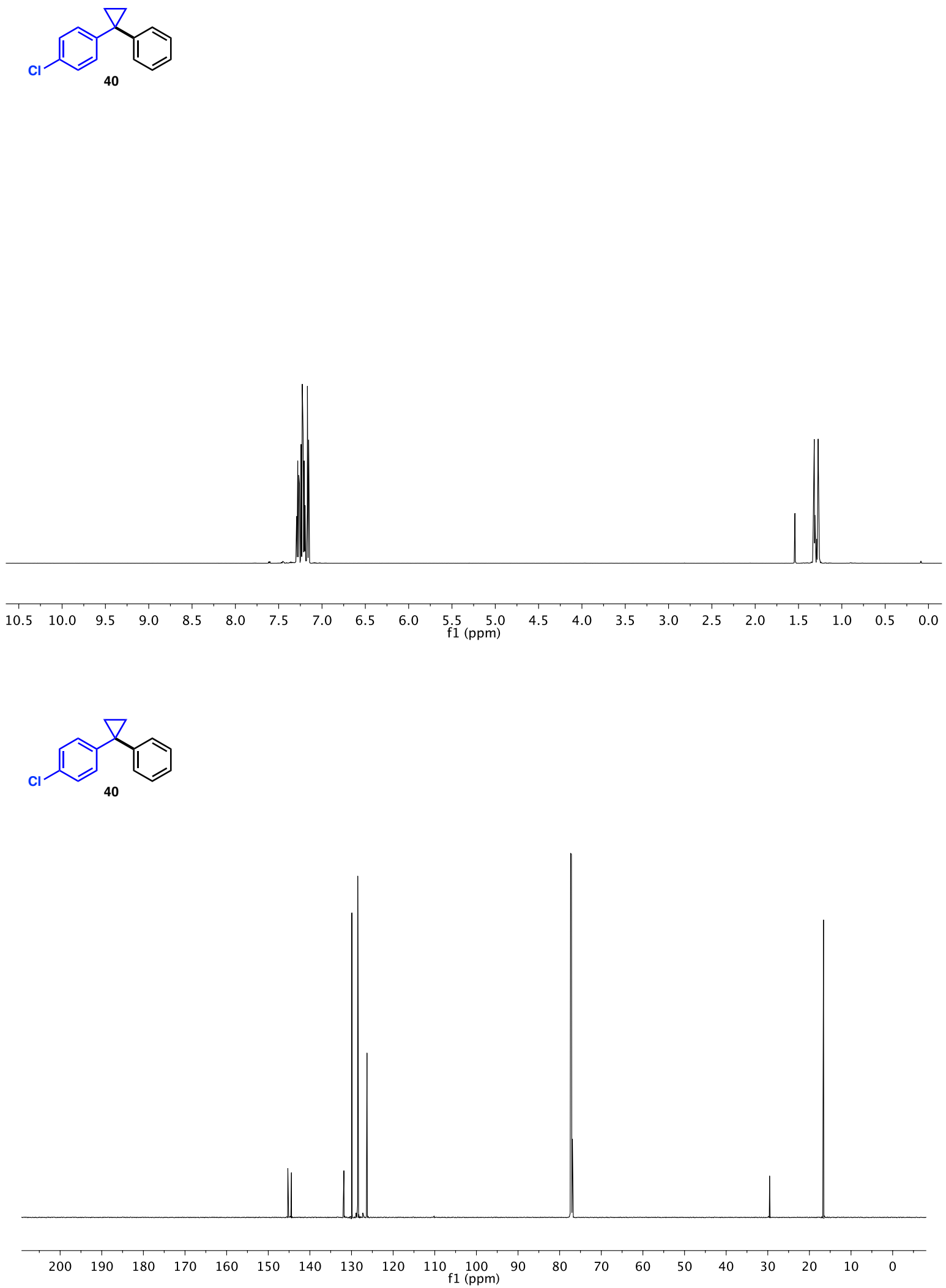

S106 

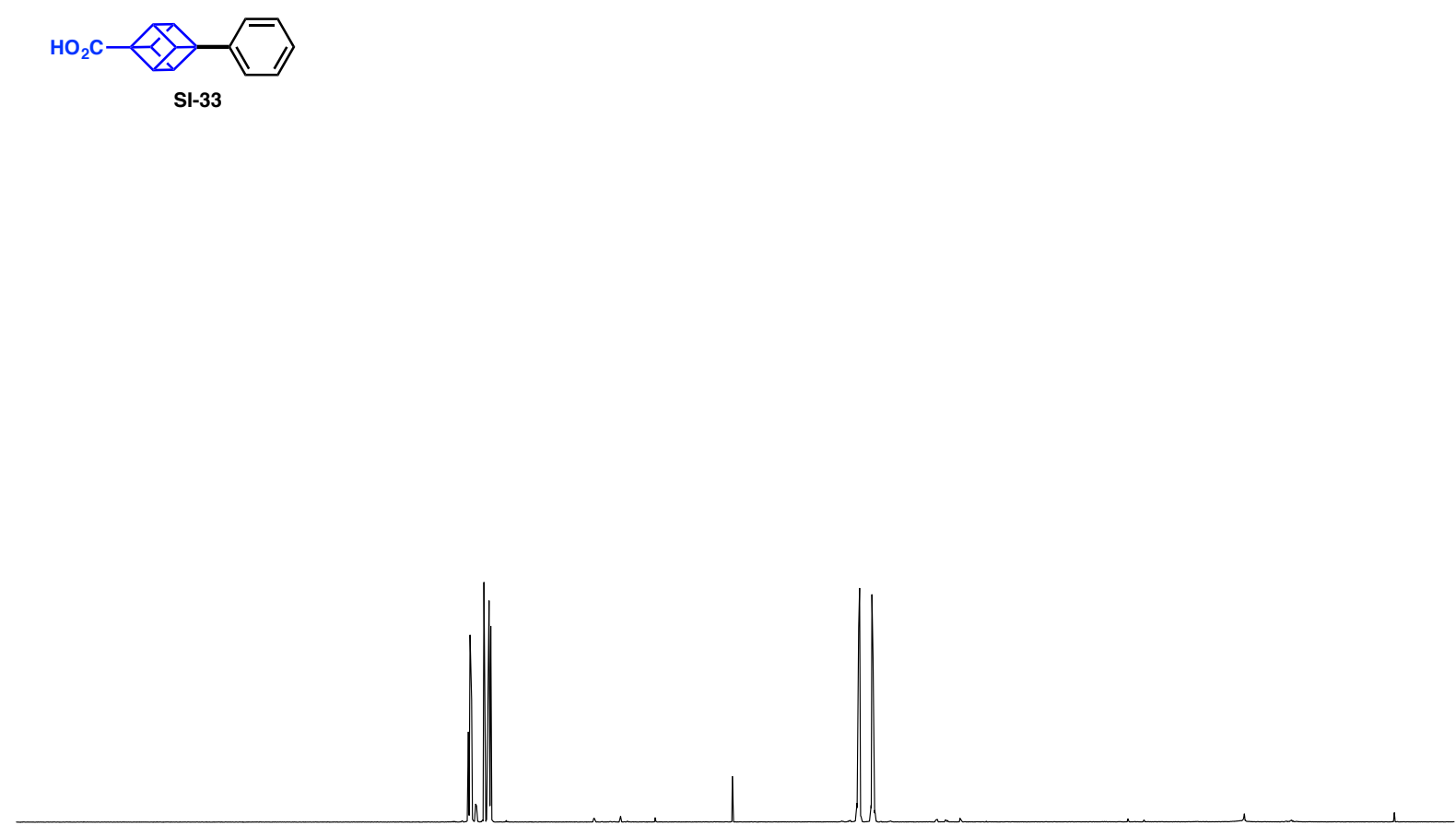

$\begin{array}{lllllllllllllllllllll}10.5 & 10.0 & 9.5 & 9.0 & 8.5 & 8.0 & 7.5 & 7.0 & 6.5 & 6.0 & \begin{array}{l}5.5 \\ \mathrm{fi}(\mathrm{ppm})\end{array} & \begin{array}{ll}5.0 \\ 4.5\end{array} & 4.0 & 3.5 & 3.0 & 2.5 & 2.0 & 1.5 & 1.0 & 0.5 & 0.0\end{array}$

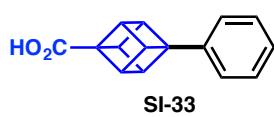

SI-33

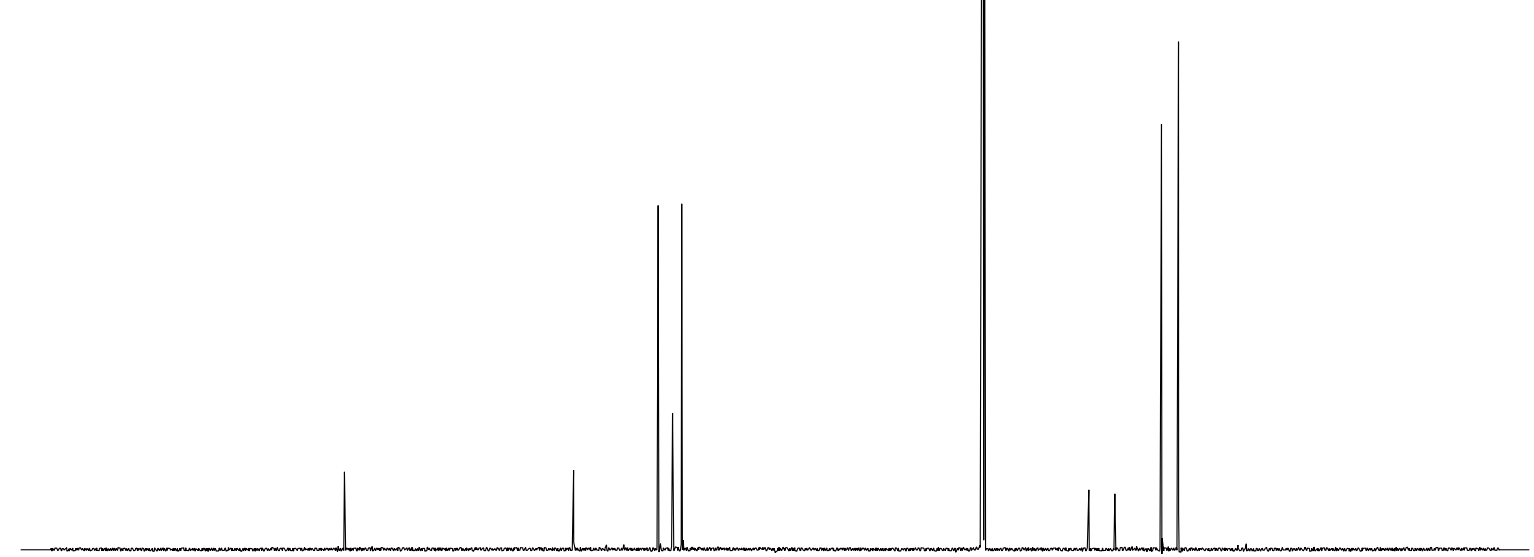

$\begin{array}{lllllllllllllllllllllll}220 & 210 & 200 & 190 & 180 & 170 & 160 & 150 & 140 & 130 & 120 & 110 & 100 & 90 & 80 & 70 & 60 & 50 & 40 & 30 & 20 & 10 & 0\end{array}$ 


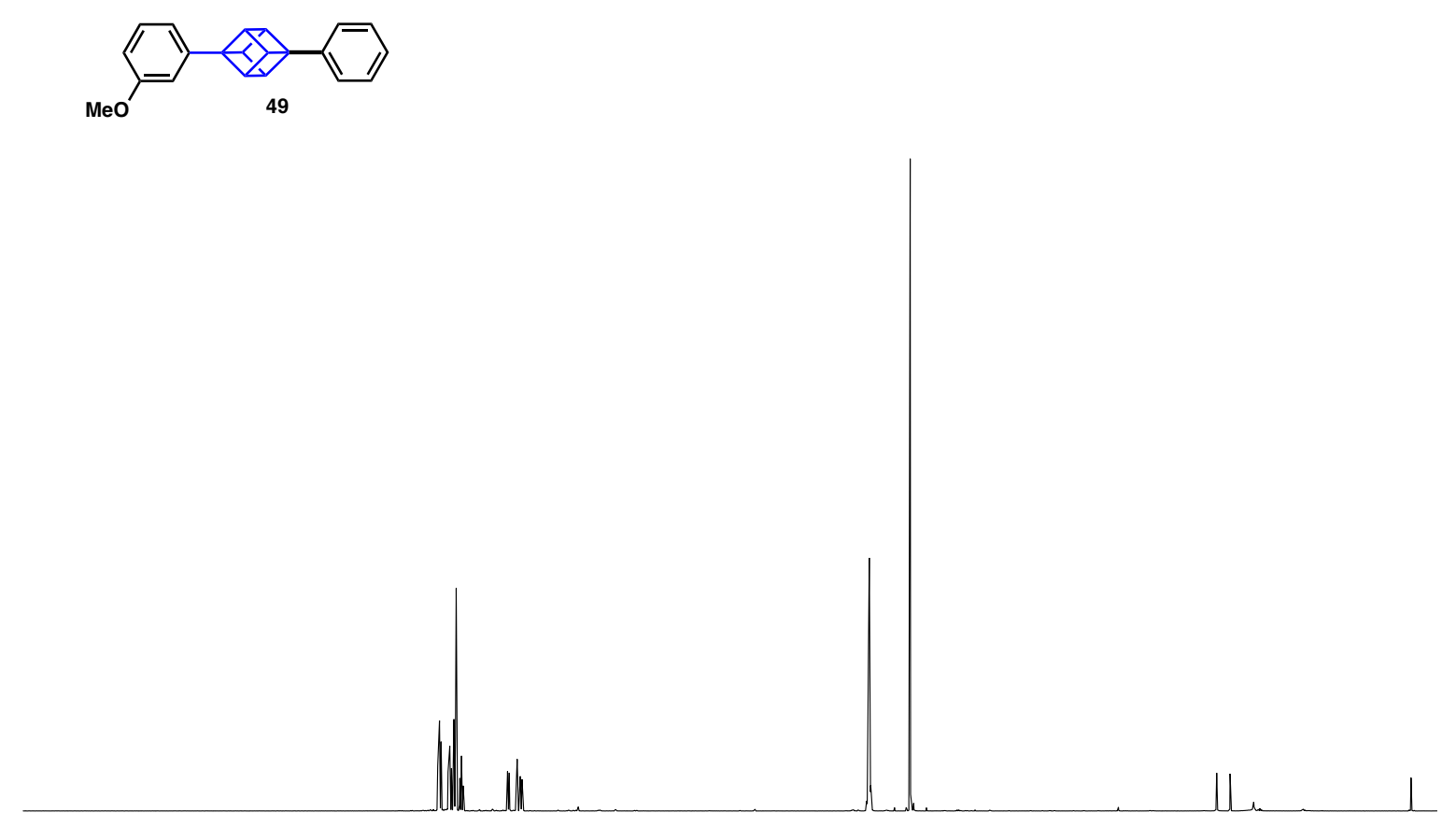

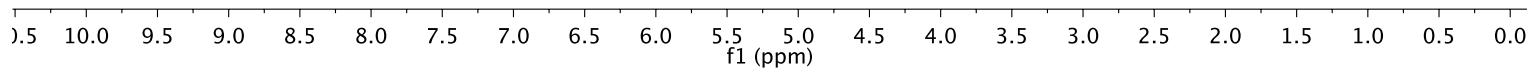
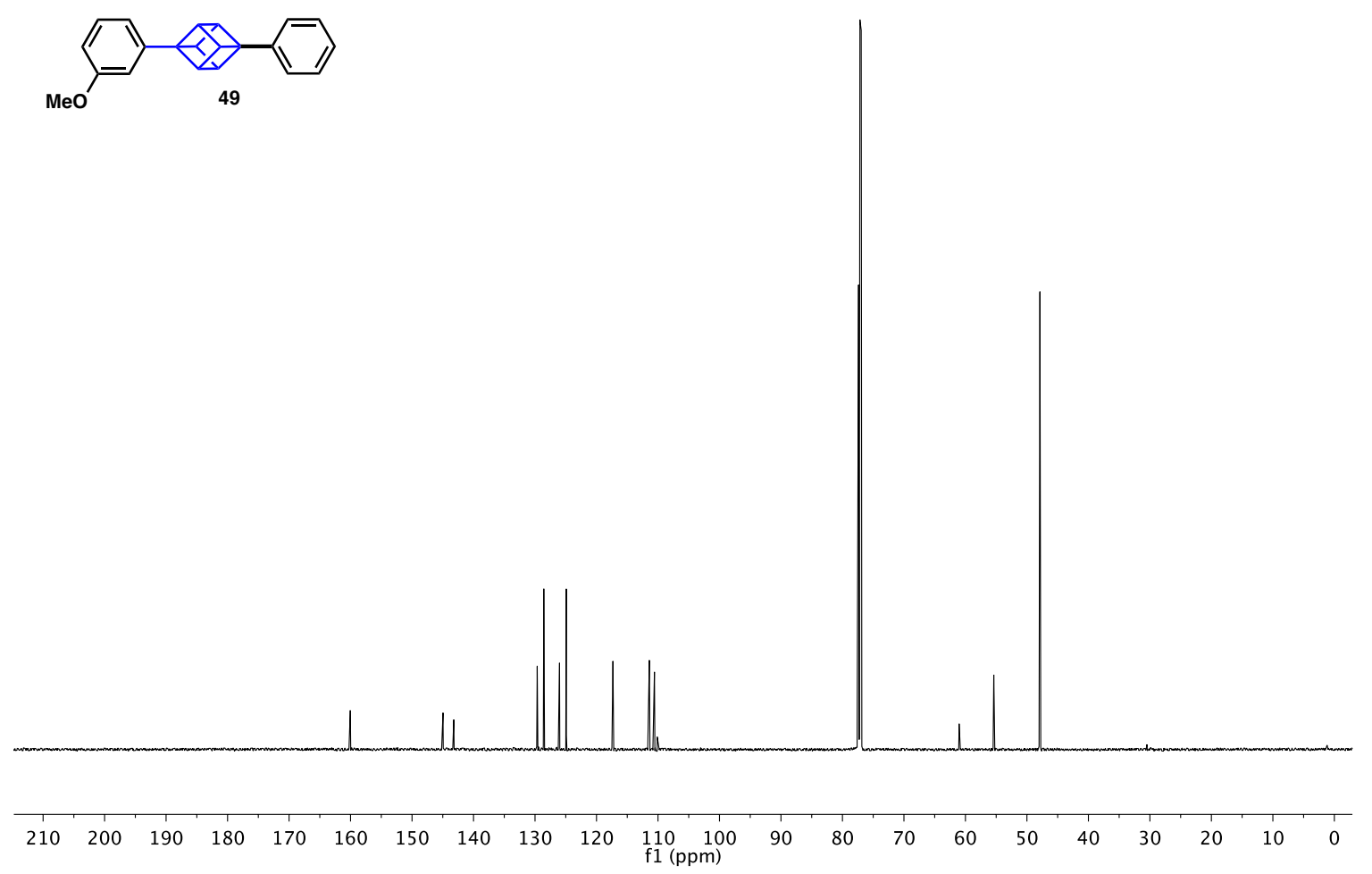

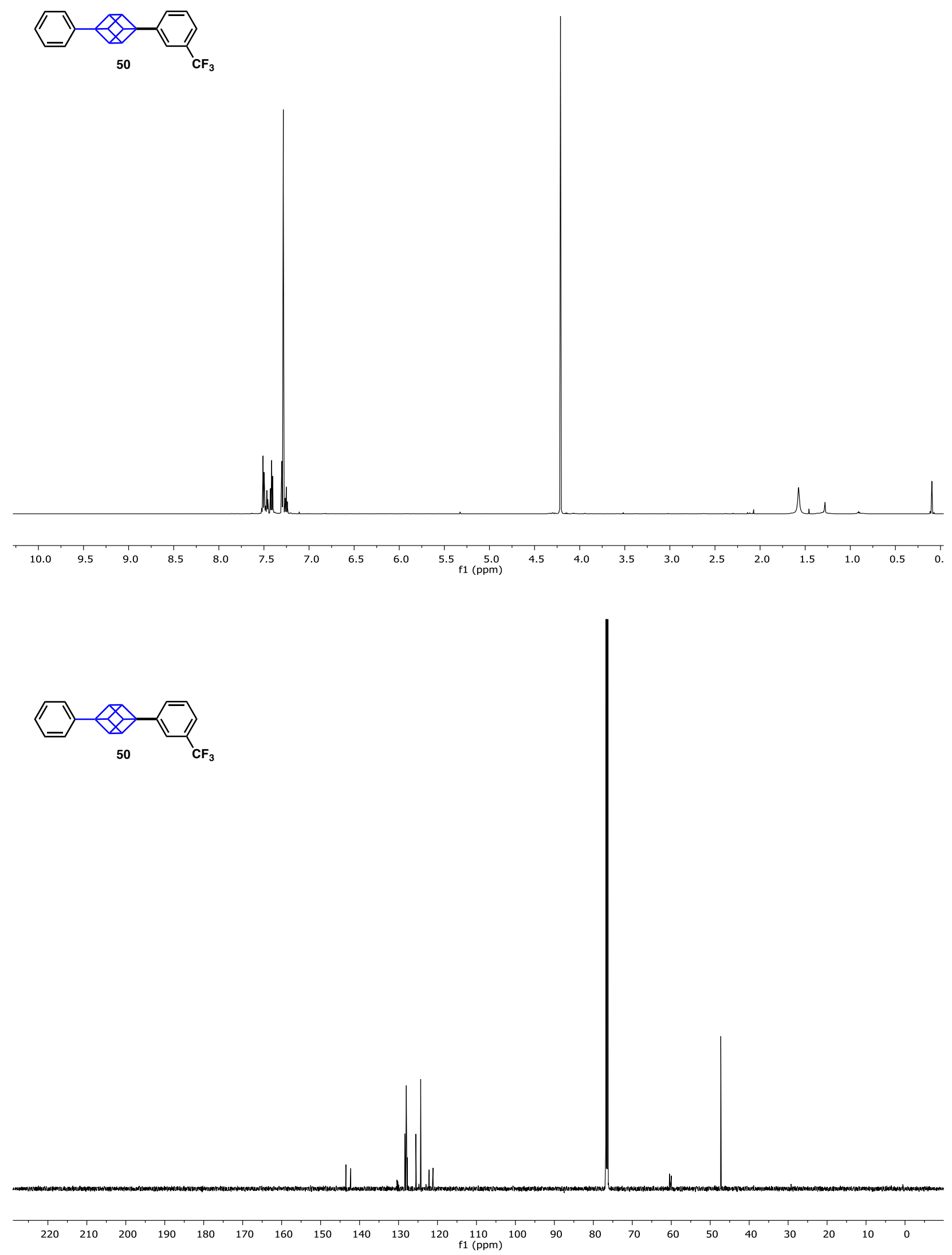

S109 

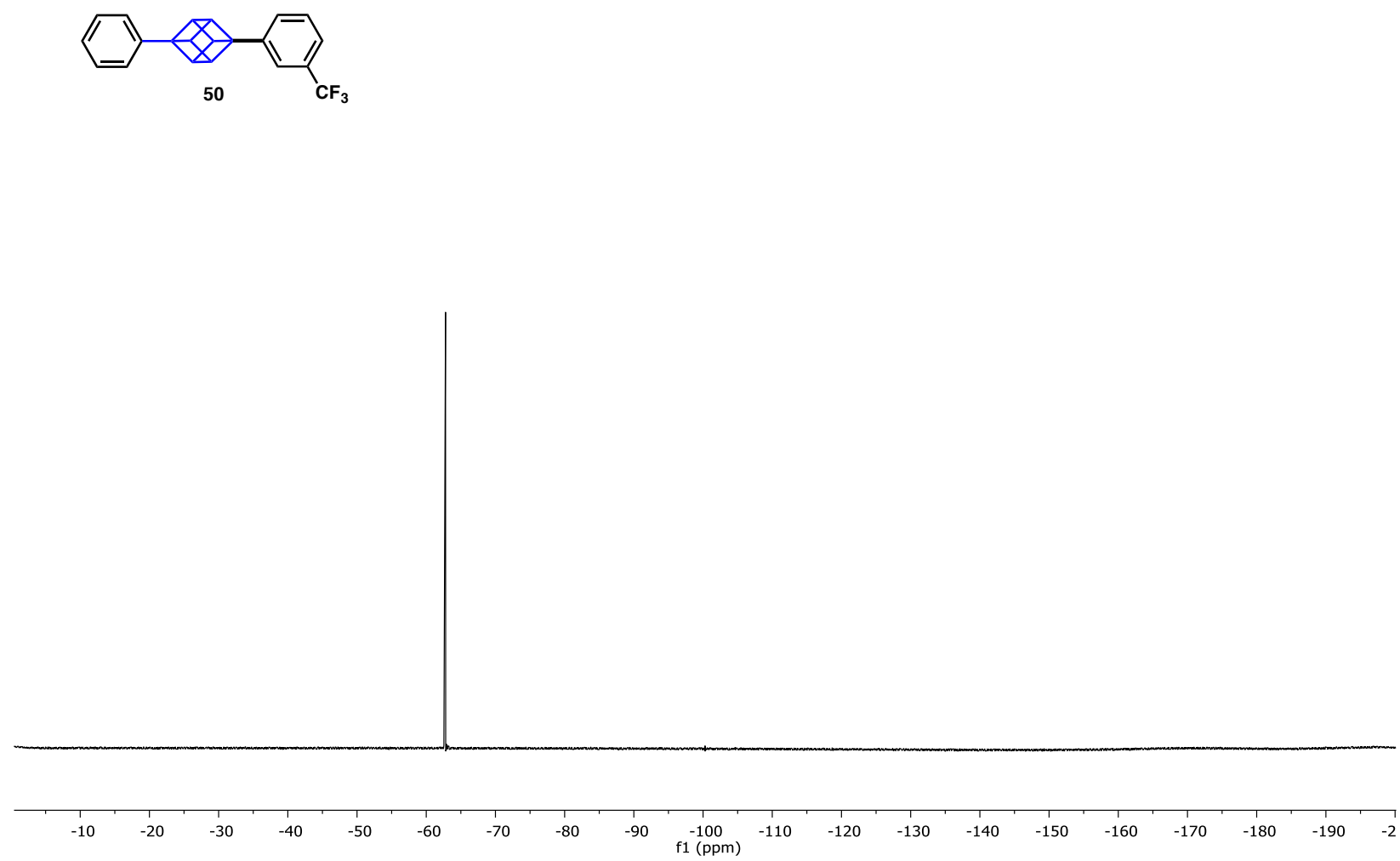


\section{Crystallographic data}

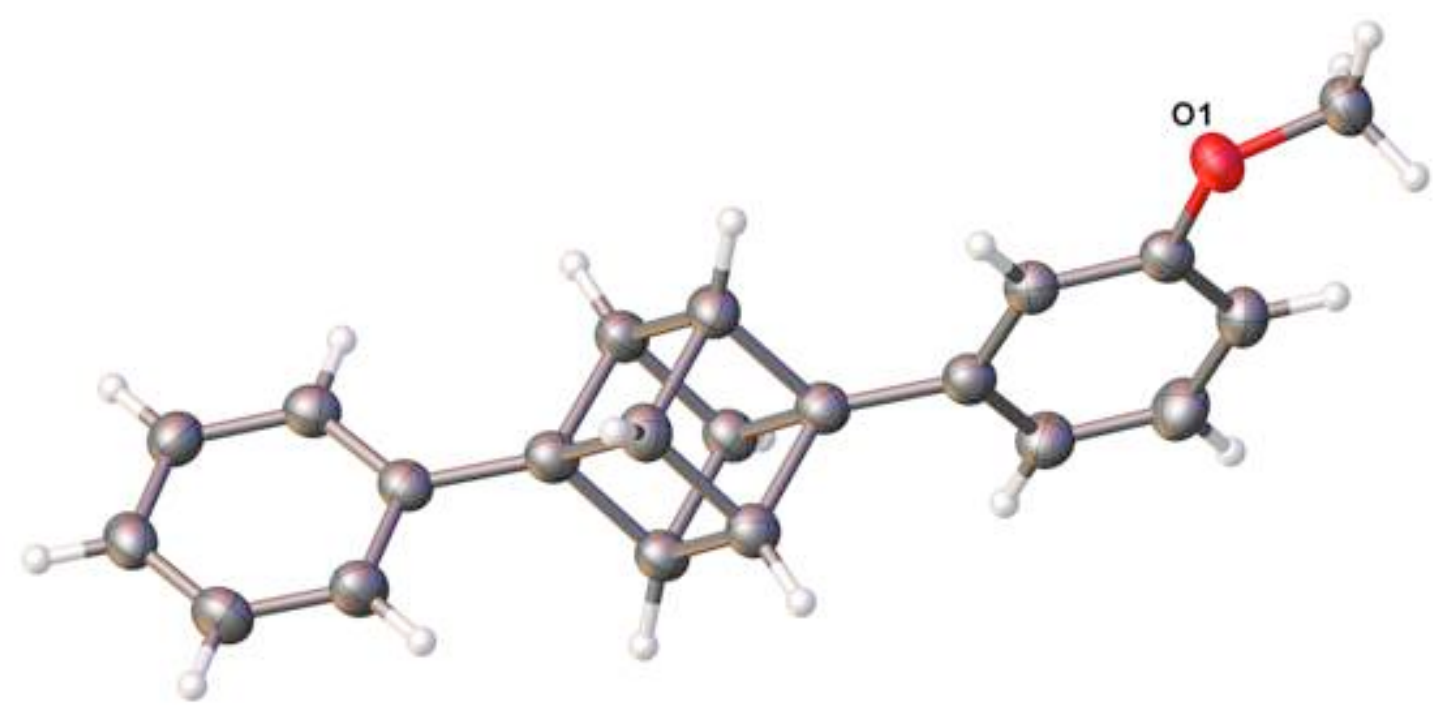

Table 1. Crystal data and structure refinement for 49.

Identification code

LAU159

Empirical formula

C21 H18 O

Formula weight

286.35

Temperature

$100.0 \mathrm{~K}$

Wavelength

$1.54178 \AA$

Crystal system

Monoclinic

Space group

C $2 / \mathrm{c}$

Unit cell dimensions

$\mathrm{a}=17.8244(5) \AA$

$\alpha=90^{\circ}$.

$\mathrm{b}=7.5148(2) \AA$

$\mathrm{c}=22.2760(6) \AA$

Volume

2940.35(14) $\AA^{3}$

$\mathrm{Z}$

8

Density (calculated)

Absorption coefficient

$1.294 \mathrm{Mg} / \mathrm{m}^{3}$

$\mathrm{F}(000)$

$0.600 \mathrm{~mm}^{-1}$

1216

Crystal size

Theta range for data collection

$0.3 \times 0.27 \times 0.14 \mathrm{~mm}^{3}$

4.028 to $68.247^{\circ}$.

Index ranges

Reflections collected

$-21<=\mathrm{h}<=21,-8<=\mathrm{k}<=8,-25<=1<=26$

Independent reflections

Completeness to theta $=67.679^{\circ}$

Absorption correction

Max. and min. transmission

Refinement method

Data / restraints / parameters

Goodness-of-fit on $\mathrm{F}^{2}$

11451

$2647[\mathrm{R}($ int $)=0.0297]$

$98.8 \%$

Semi-empirical from equivalents

0.1665 and 0.0667

Full-matrix least-squares on $\mathrm{F}^{2}$

2647 / 0 / 200

1.055

Final $R$ indices [I $>2 \operatorname{sigma}(\mathrm{I})]$

$\mathrm{R}$ indices (all data)

Extinction coefficient

Largest diff. peak and hole

$\mathrm{R} 1=0.0392, \mathrm{wR} 2=0.1052$

$\mathrm{R} 1=0.0425, \mathrm{wR} 2=0.1081$

$\mathrm{n} / \mathrm{a}$

0.219 and -0.186 e. $\AA^{-3}$

$\beta=99.790(2)^{\circ}$.

$\gamma=90^{\circ}$. 
Table 2. Atomic coordinates $\left(\mathrm{x} 10^{4}\right)$ and equivalent isotropic displacement parameters $\left(\AA^{2} \times 10^{3}\right)$ for baran588. $\mathrm{U}(\mathrm{eq})$ is defined as one third of the trace of the orthogonalized $\mathrm{U}^{\mathrm{ij}}$ tensor.

\begin{tabular}{|c|c|c|c|c|}
\hline & $\mathrm{x}$ & $\mathrm{y}$ & z & $\mathrm{U}(\mathrm{eq})$ \\
\hline $\mathrm{O}(1)$ & $8887(1)$ & $8365(1)$ & $7273(1)$ & $35(1)$ \\
\hline $\mathrm{C}(1)$ & $5377(1)$ & $289(2)$ & $3392(1)$ & $36(1)$ \\
\hline$C(2)$ & $5649(1)$ & 1953(2) & $3274(1)$ & $40(1)$ \\
\hline$C(3)$ & $5917(1)$ & $3103(2)$ & $3749(1)$ & $37(1)$ \\
\hline $\mathrm{C}(4)$ & $5924(1)$ & $2598(2)$ & $4354(1)$ & $31(1)$ \\
\hline $\mathrm{C}(5)$ & $6232(1)$ & $3846(2)$ & $4852(1)$ & $31(1)$ \\
\hline$C(6)$ & $5874(1)$ & $5705(2)$ & $4976(1)$ & $31(1)$ \\
\hline$C(7)$ & $6672(1)$ & $6599(2)$ & $5047(1)$ & $32(1)$ \\
\hline $\mathrm{C}(8)$ & 6811(1) & 6181(2) & $5756(1)$ & $31(1)$ \\
\hline $\mathrm{C}(9)$ & $7105(1)$ & $7418(2)$ & $6264(1)$ & $31(1)$ \\
\hline$C(10)$ & $7868(1)$ & $7410(2)$ & $6535(1)$ & $31(1)$ \\
\hline $\mathrm{C}(11)$ & $8125(1)$ & $8509(2)$ & 7031(1) & $31(1)$ \\
\hline$C(12)$ & 9132(1) & $9162(2)$ & $7857(1)$ & $38(1)$ \\
\hline$C(13)$ & $6602(1)$ & $8557(2)$ & 6494(1) & $37(1)$ \\
\hline$C(14)$ & $6865(1)$ & $9649(2)$ & 6983(1) & $40(1)$ \\
\hline$C(15)$ & $7626(1)$ & $9642(2)$ & $7258(1)$ & $36(1)$ \\
\hline$C(16)$ & $6370(1)$ & $3448(2)$ & $5554(1)$ & $33(1)$ \\
\hline $\mathrm{C}(17)$ & 7172(1) & 4351(2) & $5625(1)$ & $34(1)$ \\
\hline$C(18)$ & $7036(1)$ & $4769(2)$ & $4927(1)$ & $33(1)$ \\
\hline C(19) & 6004(1) & $5288(2)$ & $5672(1)$ & $32(1)$ \\
\hline$C(20)$ & $5375(1)$ & $-218(2)$ & $3988(1)$ & $36(1)$ \\
\hline$C(21)$ & $5650(1)$ & $924(2)$ & $4466(1)$ & $33(1)$ \\
\hline
\end{tabular}


Table 3. Bond lengths $[\AA]$ and angles $\left[{ }^{\circ}\right]$ for baran 588 .

\begin{tabular}{|c|c|}
\hline $\mathrm{O}(1)-\mathrm{C}(11)$ & $1.3766(15)$ \\
\hline $\mathrm{O}(1)-\mathrm{C}(12)$ & $1.4313(15)$ \\
\hline $\mathrm{C}(1)-\mathrm{H}(1)$ & 0.9500 \\
\hline$C(1)-C(2)$ & $1.382(2)$ \\
\hline$C(1)-C(20)$ & $1.3835(19)$ \\
\hline $\mathrm{C}(2)-\mathrm{H}(2)$ & 0.9500 \\
\hline$C(2)-C(3)$ & $1.3868(19)$ \\
\hline C(3)-H(3) & 0.9500 \\
\hline$C(3)-C(4)$ & $1.3969(18)$ \\
\hline$C(4)-C(5)$ & $1.4861(18)$ \\
\hline $\mathrm{C}(4)-\mathrm{C}(21)$ & $1.3875(19)$ \\
\hline$C(5)-C(6)$ & $1.5787(18)$ \\
\hline$C(5)-C(16)$ & $1.5690(17)$ \\
\hline $\mathrm{C}(5)-\mathrm{C}(18)$ & $1.5761(17)$ \\
\hline $\mathrm{C}(6)-\mathrm{H}(6)$ & 1.0000 \\
\hline$C(6)-C(7)$ & $1.5567(17)$ \\
\hline $\mathrm{C}(6)-\mathrm{C}(19)$ & $1.5595(17)$ \\
\hline $\mathrm{C}(7)-\mathrm{H}(7)$ & 1.0000 \\
\hline$C(7)-C(8)$ & $1.5886(17)$ \\
\hline $\mathrm{C}(7)-\mathrm{C}(18)$ & $1.5628(18)$ \\
\hline $\mathrm{C}(8)-\mathrm{C}(9)$ & $1.4883(18)$ \\
\hline C(8)-C(17) & $1.5666(18)$ \\
\hline C(8)-C(19) & $1.5694(17)$ \\
\hline C(9)-C(10) & $1.3913(17)$ \\
\hline $\mathrm{C}(9)-\mathrm{C}(13)$ & $1.3985(18)$ \\
\hline $\mathrm{C}(10)-\mathrm{H}(10)$ & 0.9500 \\
\hline $\mathrm{C}(10)-\mathrm{C}(11)$ & $1.3929(18)$ \\
\hline $\mathrm{C}(11)-\mathrm{C}(15)$ & $1.3876(19)$ \\
\hline $\mathrm{C}(12)-\mathrm{H}(12 \mathrm{~A})$ & 0.9800 \\
\hline $\mathrm{C}(12)-\mathrm{H}(12 \mathrm{~B})$ & 0.9800 \\
\hline $\mathrm{C}(12)-\mathrm{H}(12 \mathrm{C})$ & 0.9800 \\
\hline C(13)-H(13) & 0.9500 \\
\hline$C(13)-C(14)$ & $1.381(2)$ \\
\hline $\mathrm{C}(14)-\mathrm{H}(14)$ & 0.9500 \\
\hline$C(14)-C(15)$ & $1.3917(19)$ \\
\hline $\mathrm{C}(15)-\mathrm{H}(15)$ & 0.9500 \\
\hline
\end{tabular}




\begin{tabular}{|c|c|}
\hline C(16)-H(16) & 1.0000 \\
\hline$C(16)-C(17)$ & $1.5658(18)$ \\
\hline$C(16)-C(19)$ & $1.5695(18)$ \\
\hline $\mathrm{C}(17)-\mathrm{H}(17)$ & 1.0000 \\
\hline$C(17)-C(18)$ & $1.5646(18)$ \\
\hline C(18)-H(18) & 1.0000 \\
\hline C(19)-H(19) & 1.0000 \\
\hline $\mathrm{C}(20)-\mathrm{H}(20)$ & 0.9500 \\
\hline$C(20)-C(21)$ & $1.3895(19)$ \\
\hline $\mathrm{C}(21)-\mathrm{H}(21)$ & 0.9500 \\
\hline $\mathrm{C}(11)-\mathrm{O}(1)-\mathrm{C}(12)$ & $116.77(10)$ \\
\hline $\mathrm{C}(2)-\mathrm{C}(1)-\mathrm{H}(1)$ & 120.3 \\
\hline$C(2)-C(1)-C(20)$ & $119.32(12)$ \\
\hline $\mathrm{C}(20)-\mathrm{C}(1)-\mathrm{H}(1)$ & 120.3 \\
\hline $\mathrm{C}(1)-\mathrm{C}(2)-\mathrm{H}(2)$ & 119.8 \\
\hline $\mathrm{C}(1)-\mathrm{C}(2)-\mathrm{C}(3)$ & $120.39(12)$ \\
\hline $\mathrm{C}(3)-\mathrm{C}(2)-\mathrm{H}(2)$ & 119.8 \\
\hline $\mathrm{C}(2)-\mathrm{C}(3)-\mathrm{H}(3)$ & 119.6 \\
\hline $\mathrm{C}(2)-\mathrm{C}(3)-\mathrm{C}(4)$ & $120.75(13)$ \\
\hline $\mathrm{C}(4)-\mathrm{C}(3)-\mathrm{H}(3)$ & 119.6 \\
\hline$C(3)-C(4)-C(5)$ & $119.40(12)$ \\
\hline$C(21)-C(4)-C(3)$ & $118.33(12)$ \\
\hline$C(21)-C(4)-C(5)$ & $122.26(11)$ \\
\hline$C(4)-C(5)-C(6)$ & $125.42(10)$ \\
\hline$C(4)-C(5)-C(16)$ & $126.88(11)$ \\
\hline$C(4)-C(5)-C(18)$ & $124.76(11)$ \\
\hline$C(16)-C(5)-C(6)$ & $89.68(9)$ \\
\hline$C(16)-C(5)-C(18)$ & $89.66(9)$ \\
\hline$C(18)-C(5)-C(6)$ & $88.81(9)$ \\
\hline $\mathrm{C}(5)-\mathrm{C}(6)-\mathrm{H}(6)$ & 125.0 \\
\hline$C(7)-C(6)-C(5)$ & $90.61(9)$ \\
\hline $\mathrm{C}(7)-\mathrm{C}(6)-\mathrm{H}(6)$ & 125.0 \\
\hline$C(7)-C(6)-C(19)$ & $90.42(9)$ \\
\hline$C(19)-C(6)-C(5)$ & $89.97(9)$ \\
\hline $\mathrm{C}(19)-\mathrm{C}(6)-\mathrm{H}(6)$ & 125.0 \\
\hline $\mathrm{C}(6)-\mathrm{C}(7)-\mathrm{H}(7)$ & 125.3 \\
\hline$C(6)-C(7)-C(8)$ & $90.04(9)$ \\
\hline
\end{tabular}




\begin{tabular}{|c|c|}
\hline$C(6)-C(7)-C(18)$ & $90.09(9)$ \\
\hline $\mathrm{C}(8)-\mathrm{C}(7)-\mathrm{H}(7)$ & 125.3 \\
\hline $\mathrm{C}(18)-\mathrm{C}(7)-\mathrm{H}(7)$ & 125.3 \\
\hline$C(18)-C(7)-C(8)$ & $89.83(9)$ \\
\hline $\mathrm{C}(9)-\mathrm{C}(8)-\mathrm{C}(7)$ & $127.39(11)$ \\
\hline$C(9)-C(8)-C(17)$ & $125.98(10)$ \\
\hline C(9)-C(8)-C(19) & $123.72(11)$ \\
\hline$C(17)-C(8)-C(7)$ & $89.29(9)$ \\
\hline$C(17)-C(8)-C(19)$ & 89.93(9) \\
\hline$C(19)-C(8)-C(7)$ & $88.90(9)$ \\
\hline$C(10)-C(9)-C(8)$ & $121.30(11)$ \\
\hline$C(10)-C(9)-C(13)$ & $118.92(12)$ \\
\hline $\mathrm{C}(13)-\mathrm{C}(9)-\mathrm{C}(8)$ & $119.70(11)$ \\
\hline C(9)-C(10)-H(10) & 119.7 \\
\hline$C(9)-C(10)-C(11)$ & $120.50(12)$ \\
\hline $\mathrm{C}(11)-\mathrm{C}(10)-\mathrm{H}(10)$ & 119.7 \\
\hline $\mathrm{O}(1)-\mathrm{C}(11)-\mathrm{C}(10)$ & $115.64(11)$ \\
\hline $\mathrm{O}(1)-\mathrm{C}(11)-\mathrm{C}(15)$ & $123.79(11)$ \\
\hline$C(15)-C(11)-C(10)$ & $120.57(12)$ \\
\hline $\mathrm{O}(1)-\mathrm{C}(12)-\mathrm{H}(12 \mathrm{~A})$ & 109.5 \\
\hline $\mathrm{O}(1)-\mathrm{C}(12)-\mathrm{H}(12 \mathrm{~B})$ & 109.5 \\
\hline $\mathrm{O}(1)-\mathrm{C}(12)-\mathrm{H}(12 \mathrm{C})$ & 109.5 \\
\hline $\mathrm{H}(12 \mathrm{~A})-\mathrm{C}(12)-\mathrm{H}(12 \mathrm{~B})$ & 109.5 \\
\hline $\mathrm{H}(12 \mathrm{~A})-\mathrm{C}(12)-\mathrm{H}(12 \mathrm{C})$ & 109.5 \\
\hline $\mathrm{H}(12 \mathrm{~B})-\mathrm{C}(12)-\mathrm{H}(12 \mathrm{C})$ & 109.5 \\
\hline $\mathrm{C}(9)-\mathrm{C}(13)-\mathrm{H}(13)$ & 120.0 \\
\hline$C(14)-C(13)-C(9)$ & $120.04(12)$ \\
\hline $\mathrm{C}(14)-\mathrm{C}(13)-\mathrm{H}(13)$ & 120.0 \\
\hline $\mathrm{C}(13)-\mathrm{C}(14)-\mathrm{H}(14)$ & 119.3 \\
\hline$C(13)-C(14)-C(15)$ & $121.35(12)$ \\
\hline $\mathrm{C}(15)-\mathrm{C}(14)-\mathrm{H}(14)$ & 119.3 \\
\hline$C(11)-C(15)-C(14)$ & $118.61(12)$ \\
\hline $\mathrm{C}(11)-\mathrm{C}(15)-\mathrm{H}(15)$ & 120.7 \\
\hline $\mathrm{C}(14)-\mathrm{C}(15)-\mathrm{H}(15)$ & 120.7 \\
\hline $\mathrm{C}(5)-\mathrm{C}(16)-\mathrm{H}(16)$ & 125.2 \\
\hline$C(5)-C(16)-C(19)$ & $89.97(9)$ \\
\hline$C(17)-C(16)-C(5)$ & $90.18(9)$ \\
\hline $\mathrm{C}(17)-\mathrm{C}(16)-\mathrm{H}(16)$ & 125.2 \\
\hline
\end{tabular}




\begin{tabular}{|c|c|}
\hline$C(17)-C(16)-C(19)$ & $89.96(9)$ \\
\hline $\mathrm{C}(19)-\mathrm{C}(16)-\mathrm{H}(16)$ & 125.2 \\
\hline $\mathrm{C}(8)-\mathrm{C}(17)-\mathrm{H}(17)$ & 125.0 \\
\hline$C(16)-C(17)-C(8)$ & $90.16(9)$ \\
\hline $\mathrm{C}(16)-\mathrm{C}(17)-\mathrm{H}(17)$ & 125.0 \\
\hline $\mathrm{C}(18)-\mathrm{C}(17)-\mathrm{C}(8)$ & $90.57(10)$ \\
\hline$C(18)-C(17)-C(16)$ & $90.20(9)$ \\
\hline $\mathrm{C}(18)-\mathrm{C}(17)-\mathrm{H}(17)$ & 125.0 \\
\hline $\mathrm{C}(5)-\mathrm{C}(18)-\mathrm{H}(18)$ & 125.1 \\
\hline $\mathrm{C}(7)-\mathrm{C}(18)-\mathrm{C}(5)$ & $90.49(9)$ \\
\hline $\mathrm{C}(7)-\mathrm{C}(18)-\mathrm{C}(17)$ & $90.30(10)$ \\
\hline $\mathrm{C}(7)-\mathrm{C}(18)-\mathrm{H}(18)$ & 125.1 \\
\hline $\mathrm{C}(17)-\mathrm{C}(18)-\mathrm{C}(5)$ & $89.96(9)$ \\
\hline $\mathrm{C}(17)-\mathrm{C}(18)-\mathrm{H}(18)$ & 125.1 \\
\hline$C(6)-C(19)-C(8)$ & $90.65(9)$ \\
\hline$C(6)-C(19)-C(16)$ & $90.37(9)$ \\
\hline $\mathrm{C}(6)-\mathrm{C}(19)-\mathrm{H}(19)$ & 125.0 \\
\hline$C(8)-C(19)-C(16)$ & $89.93(9)$ \\
\hline $\mathrm{C}(8)-\mathrm{C}(19)-\mathrm{H}(19)$ & 125.0 \\
\hline $\mathrm{C}(16)-\mathrm{C}(19)-\mathrm{H}(19)$ & 125.0 \\
\hline $\mathrm{C}(1)-\mathrm{C}(20)-\mathrm{H}(20)$ & 119.8 \\
\hline $\mathrm{C}(1)-\mathrm{C}(20)-\mathrm{C}(21)$ & $120.45(13)$ \\
\hline $\mathrm{C}(21)-\mathrm{C}(20)-\mathrm{H}(20)$ & 119.8 \\
\hline$C(4)-C(21)-C(20)$ & $120.76(12)$ \\
\hline $\mathrm{C}(4)-\mathrm{C}(21)-\mathrm{H}(21)$ & 119.6 \\
\hline $\mathrm{C}(20)-\mathrm{C}(21)-\mathrm{H}(21)$ & 119.6 \\
\hline
\end{tabular}

Symmetry transformations used to generate equivalent atoms:

Table 4. Anisotropic displacement parameters $\left(\AA^{2} \times 10^{3}\right)$ for baran588. The anisotropic displacement factor exponent takes the form: $-2 \pi^{2}\left[h^{2} a^{* 2} U^{11}+\ldots+2 h k a^{*} b^{*} U^{12}\right]$

\begin{tabular}{lllllll}
\hline & $\mathrm{U}^{11}$ & $\mathrm{U}^{22}$ & $\mathrm{U}^{33}$ & $\mathrm{U}^{23}$ & $\mathrm{U}^{13}$ & $\mathrm{U}^{12}$ \\
\hline $\mathrm{O}(1)$ & $30(1)$ & $41(1)$ & $32(1)$ & $-5(1)$ & $1(1)$ & $-2(1)$ \\
$\mathrm{C}(1)$ & $32(1)$ & $38(1)$ & $36(1)$ & $-6(1)$ & $1(1)$ & $4(1)$ \\
$\mathrm{C}(2)$ & $42(1)$ & $45(1)$ & $30(1)$ & $2(1)$ & $4(1)$ & $0(1)$
\end{tabular}




\begin{tabular}{lllllll}
$\mathrm{C}(3)$ & $38(1)$ & $36(1)$ & $37(1)$ & $4(1)$ & $4(1)$ & $-4(1)$ \\
$\mathrm{C}(4)$ & $24(1)$ & $34(1)$ & $33(1)$ & $1(1)$ & $2(1)$ & $1(1)$ \\
$\mathrm{C}(5)$ & $28(1)$ & $33(1)$ & $32(1)$ & $2(1)$ & $3(1)$ & $-1(1)$ \\
$\mathrm{C}(6)$ & $28(1)$ & $33(1)$ & $31(1)$ & $2(1)$ & $2(1)$ & $-1(1)$ \\
$\mathrm{C}(7)$ & $28(1)$ & $33(1)$ & $34(1)$ & $1(1)$ & $4(1)$ & $-2(1)$ \\
$\mathrm{C}(8)$ & $26(1)$ & $33(1)$ & $33(1)$ & $1(1)$ & $2(1)$ & $1(1)$ \\
$\mathrm{C}(9)$ & $31(1)$ & $30(1)$ & $32(1)$ & $3(1)$ & $4(1)$ & $-1(1)$ \\
$\mathrm{C}(10)$ & $30(1)$ & $32(1)$ & $31(1)$ & $1(1)$ & $7(1)$ & $1(1)$ \\
$\mathrm{C}(11)$ & $31(1)$ & $32(1)$ & $31(1)$ & $3(1)$ & $4(1)$ & $-2(1)$ \\
$\mathrm{C}(12)$ & $40(1)$ & $39(1)$ & $33(1)$ & $-4(1)$ & $0(1)$ & $-4(1)$ \\
$\mathrm{C}(13)$ & $30(1)$ & $36(1)$ & $43(1)$ & $-1(1)$ & $3(1)$ & $3(1)$ \\
$\mathrm{C}(14)$ & $38(1)$ & $36(1)$ & $47(1)$ & $-5(1)$ & $10(1)$ & $5(1)$ \\
$\mathrm{C}(15)$ & $40(1)$ & $33(1)$ & $36(1)$ & $-5(1)$ & $6(1)$ & $-2(1)$ \\
$\mathrm{C}(16)$ & $32(1)$ & $32(1)$ & $32(1)$ & $2(1)$ & $1(1)$ & $-2(1)$ \\
$\mathrm{C}(17)$ & $30(1)$ & $33(1)$ & $36(1)$ & $-3(1)$ & $0(1)$ & $2(1)$ \\
$\mathrm{C}(18)$ & $27(1)$ & $36(1)$ & $36(1)$ & $-4(1)$ & $5(1)$ & $-2(1)$ \\
$\mathrm{C}(19)$ & $28(1)$ & $35(1)$ & $31(1)$ & $1(1)$ & $3(1)$ & $-1(1)$ \\
$\mathrm{C}(20)$ & $35(1)$ & $31(1)$ & $41(1)$ & $1(1)$ & $4(1)$ & $1(1)$ \\
$\mathrm{C}(21)$ & $31(1)$ & $35(1)$ & $33(1)$ & $3(1)$ & $4(1)$ & $0(1)$ \\
& & & & & & \\
\hline
\end{tabular}


Table 5. Hydrogen coordinates ( x 104) and isotropic displacement parameters $\left(\AA^{2} \times 10^{3}\right)$ for baran 588 .

\begin{tabular}{lrrrl}
\hline & $\mathrm{x}$ & $\mathrm{y}$ & $\mathrm{z}$ & $\mathrm{U}(\mathrm{eq})$ \\
& & & & \\
$\mathrm{H}(1)$ & 5193 & -498 & 3066 & 43 \\
$\mathrm{H}(2)$ & 5652 & 2311 & 2866 & 47 \\
$\mathrm{H}(3)$ & 6099 & 4248 & 3663 & 45 \\
$\mathrm{H}(6)$ & 5395 & 6206 & 4737 & 37 \\
$\mathrm{H}(7)$ & 6784 & 7759 & 4860 & 38 \\
$\mathrm{H}(10)$ & 8217 & 6648 & 6381 & 37 \\
$\mathrm{H}(12 \mathrm{~A})$ & 9063 & 10454 & 7825 & 57 \\
$\mathrm{H}(12 \mathrm{~B})$ & 9671 & 8890 & 7997 & 57 \\
$\mathrm{H}(12 \mathrm{C})$ & 8829 & 8686 & 8149 & 57 \\
$\mathrm{H}(13)$ & 6079 & 8578 & 6313 & 44 \\
$\mathrm{H}(14)$ & 6518 & 10422 & 7134 & 48 \\
$\mathrm{H}(15)$ & 7801 & 10398 & 7595 & 44 \\
$\mathrm{H}(16)$ & 6258 & 2293 & 5743 & 39 \\
$\mathrm{H}(17)$ & 7652 & 3848 & 5862 & 40 \\
$\mathrm{H}(18)$ & 7418 & 4581 & 4652 & 40 \\
$\mathrm{H}(19)$ & 5620 & 5479 & 5945 & 38 \\
$\mathrm{H}(20)$ & 5184 & -1355 & 4072 & 43 \\
$\mathrm{H}(21)$ & 5650 & 555 & 4874 & 40 \\
\hline & & & & \\
\hline
\end{tabular}




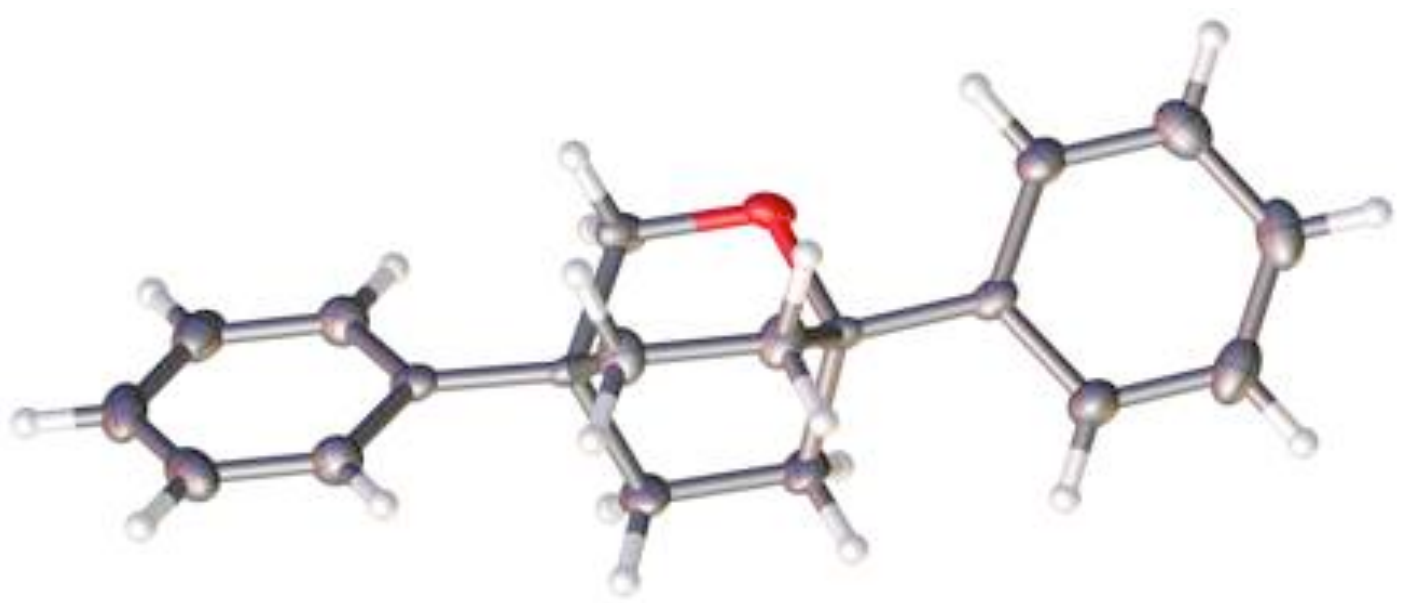

Table 1. Crystal data and structure refinement for $\mathbf{3 6}$.

Report date

Identification code

Empirical formula

Molecular formula

Formula weight

Temperature

Wavelength

Crystal system

Space group

Unit cell dimensions

Volume

Z

Density (calculated)

Absorption coefficient

$\mathrm{F}(000)$

Crystal size

Crystal color, habit

Theta range for data collection

Index ranges

Reflections collected

Independent reflections

Completeness to theta $=25.242^{\circ}$

Absorption correction

Max. and min. transmission

Refinement method
2016-06-27

baran 589

C19 H20 O

2(C9.5 H10 O0.5)

264.35

$100.0 \mathrm{~K}$

$0.71073 \AA$

Monoclinic

P $12 / \mathrm{c} 1$

$\mathrm{a}=18.758(3) \AA$

$\alpha=90^{\circ}$.

$\mathrm{b}=5.7669(8) \AA$

$\beta=110.826(4)^{\circ}$.

$\mathrm{c}=13.563(2) \AA$

$\gamma=90^{\circ}$.

1371.4(4) $\AA^{3}$

4

$1.280 \mathrm{Mg} / \mathrm{m}^{3}$

$0.077 \mathrm{~mm}^{-1}$

568

$0.33 \times 0.18 \times 0.15 \mathrm{~mm}^{3}$

colourless block

1.161 to $25.679^{\circ}$.

$-22<=\mathrm{h}<=22,-6<=\mathrm{k}<=7,-16<=\mathrm{l}<=16$

10661

$2580[\mathrm{R}(\mathrm{int})=0.0468]$

$99.8 \%$

None

0.4906 and 0.3378

Full-matrix least-squares on $\mathrm{F}^{2}$ 
Data / restraints / parameters

Goodness-of-fit on $\mathrm{F}^{2}$

Final $\mathrm{R}$ indices [I $>2 \operatorname{sigma}(\mathrm{I})]$

$\mathrm{R}$ indices (all data)

Extinction coefficient

Largest diff. peak and hole
$2580 / 24 / 188$

1.089

$\mathrm{R} 1=0.0819, \mathrm{wR} 2=0.2146$

$\mathrm{R} 1=0.0909, \mathrm{wR} 2=0.2226$

$\mathrm{n} / \mathrm{a}$

0.725 and -0.417 e. $\AA^{-3}$

Table 2. Atomic coordinates $\left(\mathrm{x} 10^{4}\right)$ and equivalent isotropic displacement parameters $\left(\AA^{2} \times 10^{3}\right)$ for Baran589. $\mathrm{U}(\mathrm{eq})$ is defined as one third of the trace of the orthogonalized $\mathrm{U}^{\mathrm{ij}}$ tensor.

\begin{tabular}{|c|c|c|c|c|}
\hline & $\mathrm{x}$ & $\mathrm{y}$ & z & $\mathrm{U}(\mathrm{eq})$ \\
\hline $\mathrm{O}(1)$ & $9634(4)$ & $6210(20)$ & $7676(9)$ & $19(2)$ \\
\hline $\mathrm{C}(1)$ & $9826(8)$ & $6230(40)$ & $7856(14)$ & $19(2)$ \\
\hline $\mathrm{C}(2)$ & $10273(2)$ & $2640(7)$ & $8662(3)$ & $25(1)$ \\
\hline$C(3)$ & $9180(2)$ & $2550(7)$ & $6959(3)$ & $23(1)$ \\
\hline $\mathrm{C}(4)$ & $9530(2)$ & $3854(6)$ & $8004(3)$ & $17(1)$ \\
\hline$C(5)$ & $8961(2)$ & $4067(6)$ & $8566(2)$ & $18(1)$ \\
\hline$C(6)$ & $8867(2)$ & $2278(7)$ & $9201(3)$ & $26(1)$ \\
\hline$C(7)$ & $8330(2)$ & $2465(8)$ & $9696(3)$ & $32(1)$ \\
\hline $\mathrm{C}(8)$ & $7880(2)$ & $4411(8)$ & $9560(3)$ & $32(1)$ \\
\hline$C(9)$ & $7963(2)$ & $6197(7)$ & $8934(3)$ & $31(1)$ \\
\hline$C(10)$ & $8497(2)$ & $6035(7)$ & $8437(3)$ & $24(1)$ \\
\hline $\mathrm{O}(2)$ & $5367(8)$ & $4190(40)$ & $3009(17)$ & $31(2)$ \\
\hline $\mathrm{C}(11)$ & $5215(14)$ & $4190(60)$ & $3010(30)$ & $31(2)$ \\
\hline$C(12)$ & $5804(2)$ & $495(8)$ & $2708(3)$ & $33(1)$ \\
\hline $\mathrm{C}(13)$ & $4756(2)$ & $696(7)$ & $3429(3)$ & $30(1)$ \\
\hline$C(14)$ & $5493(2)$ & $1788(7)$ & $3446(3)$ & $21(1)$ \\
\hline$C(15)$ & $6062(2)$ & $2000(7)$ & $4575(3)$ & $23(1)$ \\
\hline$C(16)$ & $6567(2)$ & $211(7)$ & $5001(3)$ & $26(1)$ \\
\hline$C(17)$ & $7064(2)$ & $275(8)$ & $6041(3)$ & $31(1)$ \\
\hline$C(18)$ & $7065(2)$ & $2160(8)$ & $6671(3)$ & $31(1)$ \\
\hline $\mathrm{C}(19)$ & $6566(2)$ & $3967(7)$ & $6249(3)$ & $32(1)$ \\
\hline$C(20)$ & $6066(2)$ & $3878(7)$ & $5207(3)$ & $30(1)$ \\
\hline
\end{tabular}


Table 3. Bond lengths $[\AA]$ and angles $\left[^{\circ}\right]$ for Baran589.

\begin{tabular}{|c|c|}
\hline $\begin{array}{l}\mathrm{O}(1)-\mathrm{C}(4) \\
\mathrm{C}(1)-\mathrm{O}(1) \# 1\end{array}$ & $\begin{array}{l}1.465(14) \\
1.437(10)\end{array}$ \\
\hline $\mathrm{C}(1)-\mathrm{H}(1 \mathrm{~A})$ & $0.999(16)$ \\
\hline $\mathrm{C}(1)-\mathrm{H}(1 \mathrm{~B})$ & $0.980(18)$ \\
\hline$C(1)-C(4)$ & $1.52(2)$ \\
\hline $\mathrm{C}(2)-\mathrm{H}(2 \mathrm{~A})$ & 0.9900 \\
\hline $\mathrm{C}(2)-\mathrm{H}(2 \mathrm{~B})$ & 0.9900 \\
\hline $\mathrm{C}(2)-\mathrm{C}(3) \# 1$ & $1.544(5)$ \\
\hline$C(2)-C(4)$ & $1.531(4)$ \\
\hline$C(3)-C(2) \# 1$ & $1.544(5)$ \\
\hline $\mathrm{C}(3)-\mathrm{H}(3 \mathrm{~A})$ & 0.9900 \\
\hline $\mathrm{C}(3)-\mathrm{H}(3 \mathrm{~B})$ & 0.9900 \\
\hline$C(3)-C(4)$ & $1.531(4)$ \\
\hline$C(4)-C(5)$ & $1.521(5)$ \\
\hline$C(5)-C(6)$ & $1.395(5)$ \\
\hline$C(5)-C(10)$ & $1.402(5)$ \\
\hline $\mathrm{C}(6)-\mathrm{H}(6)$ & 0.9500 \\
\hline$C(6)-C(7)$ & $1.399(6)$ \\
\hline $\mathrm{C}(7)-\mathrm{H}(7)$ & 0.9500 \\
\hline$C(7)-C(8)$ & $1.377(6)$ \\
\hline $\mathrm{C}(8)-\mathrm{H}(8)$ & 0.9500 \\
\hline $\mathrm{C}(8)-\mathrm{C}(9)$ & $1.378(6)$ \\
\hline $\mathrm{C}(9)-\mathrm{H}(9)$ & 0.9500 \\
\hline $\mathrm{C}(9)-\mathrm{C}(10)$ & $1.396(5)$ \\
\hline $\mathrm{C}(10)-\mathrm{H}(10)$ & 0.9500 \\
\hline $\mathrm{O}(2)-\mathrm{C}(14)$ & $1.49(2)$ \\
\hline $\mathrm{C}(11)-\mathrm{O}(2) \# 2$ & $1.428(12)$ \\
\hline $\mathrm{C}(11)-\mathrm{H}(11 \mathrm{~A})$ & $0.99(3)$ \\
\hline $\mathrm{C}(11)-\mathrm{H}(11 \mathrm{~B})$ & $1.00(3)$ \\
\hline$C(11)-C(14)$ & $1.52(4)$ \\
\hline $\mathrm{C}(12)-\mathrm{H}(12 \mathrm{~A})$ & 0.9900 \\
\hline $\mathrm{C}(12)-\mathrm{H}(12 \mathrm{~B})$ & 0.9900 \\
\hline$C(12)-C(13) \# 2$ & $1.531(5)$ \\
\hline$C(12)-C(14)$ & $1.520(5)$ \\
\hline $\mathrm{C}(13)-\mathrm{C}(12) \# 2$ & $1.531(5)$ \\
\hline $\mathrm{C}(13)-\mathrm{H}(13 \mathrm{~A})$ & 0.9900 \\
\hline $\mathrm{C}(13)-\mathrm{H}(13 \mathrm{~B})$ & 0.9900 \\
\hline$C(13)-C(14)$ & $1.513(5)$ \\
\hline
\end{tabular}




\begin{tabular}{|c|c|}
\hline$C(14)-C(15)$ & $1.528(5)$ \\
\hline $\mathrm{C}(15)-\mathrm{C}(16)$ & $1.382(5)$ \\
\hline$C(15)-C(20)$ & $1.380(6)$ \\
\hline $\mathrm{C}(16)-\mathrm{H}(16)$ & 0.9500 \\
\hline$C(16)-C(17)$ & $1.387(5)$ \\
\hline $\mathrm{C}(17)-\mathrm{H}(17)$ & 0.9500 \\
\hline$C(17)-C(18)$ & $1.381(6)$ \\
\hline C(18)-H(18) & 0.9500 \\
\hline C(18)-C(19) & $1.382(6)$ \\
\hline C(19)-H(19) & 0.9500 \\
\hline$C(19)-C(20)$ & $1.392(5)$ \\
\hline $\mathrm{C}(20)-\mathrm{H}(20)$ & 0.9500 \\
\hline $\mathrm{O}(1) \# 1-\mathrm{C}(1)-\mathrm{C}(4)$ & $114.6(18)$ \\
\hline $\mathrm{H}(1 \mathrm{~A})-\mathrm{C}(1)-\mathrm{H}(1 \mathrm{~B})$ & $107.7(19)$ \\
\hline $\mathrm{C}(4)-\mathrm{C}(1)-\mathrm{H}(1 \mathrm{~A})$ & $107.7(12)$ \\
\hline $\mathrm{C}(4)-\mathrm{C}(1)-\mathrm{H}(1 \mathrm{~B})$ & $108.8(16)$ \\
\hline $\mathrm{H}(2 \mathrm{~A})-\mathrm{C}(2)-\mathrm{H}(2 \mathrm{~B})$ & 108.2 \\
\hline $\mathrm{C}(3) \# 1-\mathrm{C}(2)-\mathrm{H}(2 \mathrm{~A})$ & 109.7 \\
\hline $\mathrm{C}(3) \# 1-\mathrm{C}(2)-\mathrm{H}(2 \mathrm{~B})$ & 109.7 \\
\hline $\mathrm{C}(4)-\mathrm{C}(2)-\mathrm{H}(2 \mathrm{~A})$ & 109.7 \\
\hline $\mathrm{C}(4)-\mathrm{C}(2)-\mathrm{H}(2 \mathrm{~B})$ & 109.7 \\
\hline $\mathrm{C}(4)-\mathrm{C}(2)-\mathrm{C}(3) \# 1$ & $109.9(3)$ \\
\hline $\mathrm{C}(2) \# 1-\mathrm{C}(3)-\mathrm{H}(3 \mathrm{~A})$ & 109.7 \\
\hline $\mathrm{C}(2) \# 1-\mathrm{C}(3)-\mathrm{H}(3 \mathrm{~B})$ & 109.7 \\
\hline $\mathrm{H}(3 \mathrm{~A})-\mathrm{C}(3)-\mathrm{H}(3 \mathrm{~B})$ & 108.2 \\
\hline $\mathrm{C}(4)-\mathrm{C}(3)-\mathrm{C}(2) \# 1$ & $109.9(3)$ \\
\hline $\mathrm{C}(4)-\mathrm{C}(3)-\mathrm{H}(3 \mathrm{~A})$ & 109.7 \\
\hline $\mathrm{C}(4)-\mathrm{C}(3)-\mathrm{H}(3 \mathrm{~B})$ & 109.7 \\
\hline $\mathrm{O}(1)-\mathrm{C}(4)-\mathrm{C}(2)$ & $114.2(4)$ \\
\hline $\mathrm{O}(1)-\mathrm{C}(4)-\mathrm{C}(3)$ & $103.5(5)$ \\
\hline $\mathrm{O}(1)-\mathrm{C}(4)-\mathrm{C}(5)$ & $105.7(5)$ \\
\hline$C(1)-C(4)-C(2)$ & $101.0(6)$ \\
\hline$C(1)-C(4)-C(3)$ & $112.1(7)$ \\
\hline $\mathrm{C}(1)-\mathrm{C}(4)-\mathrm{C}(5)$ & $110.4(8)$ \\
\hline$C(3)-C(4)-C(2)$ & $108.5(3)$ \\
\hline$C(5)-C(4)-C(2)$ & $113.6(3)$ \\
\hline$C(5)-C(4)-C(3)$ & $110.9(3)$ \\
\hline$C(6)-C(5)-C(4)$ & $120.9(3)$ \\
\hline$C(6)-C(5)-C(10)$ & $117.8(3)$ \\
\hline
\end{tabular}




\begin{tabular}{|c|c|}
\hline$C(10)-C(5)-C(4)$ & $121.2(3)$ \\
\hline $\mathrm{C}(5)-\mathrm{C}(6)-\mathrm{H}(6)$ & 119.7 \\
\hline$C(5)-C(6)-C(7)$ & $120.6(4)$ \\
\hline $\mathrm{C}(7)-\mathrm{C}(6)-\mathrm{H}(6)$ & 119.7 \\
\hline $\mathrm{C}(6)-\mathrm{C}(7)-\mathrm{H}(7)$ & 119.7 \\
\hline$C(8)-C(7)-C(6)$ & $120.7(4)$ \\
\hline $\mathrm{C}(8)-\mathrm{C}(7)-\mathrm{H}(7)$ & 119.7 \\
\hline $\mathrm{C}(7)-\mathrm{C}(8)-\mathrm{H}(8)$ & 120.2 \\
\hline$C(7)-C(8)-C(9)$ & $119.6(4)$ \\
\hline $\mathrm{C}(9)-\mathrm{C}(8)-\mathrm{H}(8)$ & 120.2 \\
\hline $\mathrm{C}(8)-\mathrm{C}(9)-\mathrm{H}(9)$ & 119.8 \\
\hline $\mathrm{C}(8)-\mathrm{C}(9)-\mathrm{C}(10)$ & $120.3(4)$ \\
\hline $\mathrm{C}(10)-\mathrm{C}(9)-\mathrm{H}(9)$ & 119.8 \\
\hline $\mathrm{C}(5)-\mathrm{C}(10)-\mathrm{H}(10)$ & 119.5 \\
\hline$C(9)-C(10)-C(5)$ & $120.9(3)$ \\
\hline $\mathrm{C}(9)-\mathrm{C}(10)-\mathrm{H}(10)$ & 119.5 \\
\hline $\mathrm{O}(2) \# 2-\mathrm{C}(11)-\mathrm{C}(14)$ & $114(3)$ \\
\hline $\mathrm{H}(11 \mathrm{~A})-\mathrm{C}(11)-\mathrm{H}(11 \mathrm{~B})$ & $107(3)$ \\
\hline $\mathrm{C}(14)-\mathrm{C}(11)-\mathrm{H}(11 \mathrm{~A})$ & $109(3)$ \\
\hline $\mathrm{C}(14)-\mathrm{C}(11)-\mathrm{H}(11 \mathrm{~B})$ & $109(2)$ \\
\hline $\mathrm{H}(12 \mathrm{~A})-\mathrm{C}(12)-\mathrm{H}(12 \mathrm{~B})$ & 108.2 \\
\hline $\mathrm{C}(13) \# 2-\mathrm{C}(12)-\mathrm{H}(12 \mathrm{~A})$ & 109.7 \\
\hline $\mathrm{C}(13) \# 2-\mathrm{C}(12)-\mathrm{H}(12 \mathrm{~B})$ & 109.7 \\
\hline $\mathrm{C}(14)-\mathrm{C}(12)-\mathrm{H}(12 \mathrm{~A})$ & 109.7 \\
\hline $\mathrm{C}(14)-\mathrm{C}(12)-\mathrm{H}(12 \mathrm{~B})$ & 109.7 \\
\hline$C(14)-C(12)-C(13) \# 2$ & $109.8(3)$ \\
\hline$C(12) \# 2-C(13)-H(13 A)$ & 109.6 \\
\hline $\mathrm{C}(12) \# 2-\mathrm{C}(13)-\mathrm{H}(13 \mathrm{~B})$ & 109.6 \\
\hline $\mathrm{H}(13 \mathrm{~A})-\mathrm{C}(13)-\mathrm{H}(13 \mathrm{~B})$ & 108.2 \\
\hline $\mathrm{C}(14)-\mathrm{C}(13)-\mathrm{C}(12) \# 2$ & $110.1(3)$ \\
\hline $\mathrm{C}(14)-\mathrm{C}(13)-\mathrm{H}(13 \mathrm{~A})$ & 109.6 \\
\hline $\mathrm{C}(14)-\mathrm{C}(13)-\mathrm{H}(13 \mathrm{~B})$ & 109.6 \\
\hline $\mathrm{O}(2)-\mathrm{C}(14)-\mathrm{C}(12)$ & $103.8(9)$ \\
\hline $\mathrm{O}(2)-\mathrm{C}(14)-\mathrm{C}(13)$ & $111.4(6)$ \\
\hline $\mathrm{O}(2)-\mathrm{C}(14)-\mathrm{C}(15)$ & $106.2(8)$ \\
\hline$C(11)-C(14)-C(15)$ & $109.6(12)$ \\
\hline $\mathrm{C}(12)-\mathrm{C}(14)-\mathrm{C}(11)$ & $110.6(13)$ \\
\hline$C(12)-C(14)-C(15)$ & $113.8(3)$ \\
\hline $\mathrm{C}(13)-\mathrm{C}(14)-\mathrm{C}(11)$ & $100.7(10)$ \\
\hline$C(13)-C(14)-C(12)$ & $110.4(3)$ \\
\hline
\end{tabular}




$\begin{array}{ll}\mathrm{C}(13)-\mathrm{C}(14)-\mathrm{C}(15) & 110.9(3) \\ \mathrm{C}(16)-\mathrm{C}(15)-\mathrm{C}(14) & 119.4(3) \\ \mathrm{C}(20)-\mathrm{C}(15)-\mathrm{C}(14) & 122.3(3) \\ \mathrm{C}(20)-\mathrm{C}(15)-\mathrm{C}(16) & 118.3(3) \\ \mathrm{C}(15)-\mathrm{C}(16)-\mathrm{H}(16) & 119.4 \\ \mathrm{C}(15)-\mathrm{C}(16)-\mathrm{C}(17) & 121.2(4) \\ \mathrm{C}(17)-\mathrm{C}(16)-\mathrm{H}(16) & 119.4 \\ \mathrm{C}(16)-\mathrm{C}(17)-\mathrm{H}(17) & 119.9 \\ \mathrm{C}(18)-\mathrm{C}(17)-\mathrm{C}(16) & 120.2(4) \\ \mathrm{C}(18)-\mathrm{C}(17)-\mathrm{H}(17) & 119.9 \\ \mathrm{C}(17)-\mathrm{C}(18)-\mathrm{H}(18) & 120.5 \\ \mathrm{C}(17)-\mathrm{C}(18)-\mathrm{C}(19) & 119.1(4) \\ \mathrm{C}(19)-\mathrm{C}(18)-\mathrm{H}(18) & 120.5 \\ \mathrm{C}(18)-\mathrm{C}(19)-\mathrm{H}(19) & 119.8 \\ \mathrm{C}(18)-\mathrm{C}(19)-\mathrm{C}(20) & 120.3(4) \\ \mathrm{C}(20)-\mathrm{C}(19)-\mathrm{H}(19) & 119.8 \\ \mathrm{C}(15)-\mathrm{C}(20)-\mathrm{C}(19) & 120.9(4) \\ \mathrm{C}(15)-\mathrm{C}(20)-\mathrm{H}(20) & 119.6 \\ \mathrm{C}(19)-\mathrm{C}(20)-\mathrm{H}(20) & 119.6 \\ & \end{array}$

Table 1. Crystal data and structure refinement for $\mathbf{5 0 .}$

Report date

Identification code

Empirical formula

Molecular formula

Formula weight

Temperature

Wavelength

Crystal system

Space group
2016-06-28

baran 585

C21 H15 F3

C21 H15 F3

324.33

$125 \mathrm{~K}$

$1.54178 \AA$

Orthorhombic

Pna21 
Unit cell dimensions

Volume

Z

Density (calculated)

Absorption coefficient

$\mathrm{F}(000)$

Crystal size

Crystal color, habit

Theta range for data collection

Index ranges

Reflections collected

Independent reflections

Completeness to theta $=67.679^{\circ}$

Absorption correction

Max. and min. transmission

Refinement method

Data / restraints / parameters

Goodness-of-fit on $\mathrm{F}^{2}$

Final R indices [I $>2 \operatorname{sigma}(\mathrm{I})]$

$\mathrm{R}$ indices (all data)

Absolute structure parameter

Extinction coefficient

Largest diff. peak and hole

$$
\begin{array}{ll}
\mathrm{a}=16.6380(7) \AA & \alpha=90^{\circ} . \\
\mathrm{b}=5.4810(3) \AA & \beta=90^{\circ} . \\
\mathrm{c}=33.4803(15) \AA & \gamma=90^{\circ} .
\end{array}
$$$$
\text { 3053.2(3) } \AA^{3}
$$$$
8
$$

$1.411 \mathrm{Mg} / \mathrm{m}^{3}$

$0.889 \mathrm{~mm}^{-1}$

1344

$0.3 \times 0.07 \times 0.07 \mathrm{~mm}^{3}$

colorless needle

2.639 to $68.749^{\circ}$.

$-19<=\mathrm{h}<=17,-6<=\mathrm{k}<=6,-40<=\mathrm{l}<=40$

38783

$5611[\mathrm{R}(\mathrm{int})=0.0706]$

$99.8 \%$

Semi-empirical from equivalents

0.6612 and 0.5284

Full-matrix least-squares on $\mathrm{F}^{2}$

$5611 / 1 / 434$

1.050

$\mathrm{R} 1=0.0481, \mathrm{wR} 2=0.1186$

$\mathrm{R} 1=0.0572, \mathrm{wR} 2=0.1244$

0.5

$0.0012(2)$

0.261 and -0.229 e. $\AA^{-3}$

Table 2. Atomic coordinates $\left(\mathrm{x} 10^{4}\right)$ and equivalent isotropic displacement parameters $\left(\AA^{2} \times 10^{3}\right)$ for Baran585_a. U(eq) is defined as one third of the trace of the orthogonalized $U^{i j}$ tensor.

\begin{tabular}{lcccc}
\hline & $\mathrm{x}$ & $\mathrm{y}$ & $\mathrm{z}$ & $\mathrm{U}(\mathrm{eq})$ \\
\hline $\mathrm{F}\left(1^{\prime}\right)$ & $4979(3)$ & $5347(7)$ & $4816(1)$ & $69(1)$ \\
$\mathrm{F}\left(2^{\prime}\right)$ & $5487(2)$ & $8574(7)$ & $5084(1)$ & $57(1)$ \\
$\mathrm{F}\left(3^{\prime}\right)$ & $4251(2)$ & $7691(6)$ & $5171(1)$ & $54(1)$ \\
$\mathrm{C}\left(1^{\prime}\right)$ & $4988(3)$ & $6669(10)$ & $5149(2)$ & $38(1)$ \\
$\mathrm{C}\left(2^{\prime}\right)$ & $5197(3)$ & $5252(9)$ & $5506(1)$ & $25(1)$ \\
$\mathrm{C}\left(3^{\prime}\right)$ & $5891(2)$ & $5666(8)$ & $5721(1)$ & $23(1)$
\end{tabular}




\begin{tabular}{|c|c|c|c|c|}
\hline$C\left(4^{\prime}\right)$ & 6099(2) & $4204(8)$ & $6048(1)$ & 19(1) \\
\hline$C\left(5^{\prime}\right)$ & $5582(2)$ & $2315(7)$ & $6154(1)$ & $22(1)$ \\
\hline$C\left(6^{\prime}\right)$ & $4880(3)$ & 1896(9) & $5942(1)$ & $28(1)$ \\
\hline$C\left(7^{\prime}\right)$ & 4683(3) & $3334(9)$ & $5615(1)$ & $29(1)$ \\
\hline$C\left(8^{\prime}\right)$ & $6874(2)$ & $4608(7)$ & $6259(1)$ & $20(1)$ \\
\hline $\mathrm{C}\left(9^{\prime}\right)$ & $7232(2)$ & 7157(8) & $6370(1)$ & $24(1)$ \\
\hline$C\left(10^{\prime}\right)$ & $7477(2)$ & $6168(8)$ & $6786(1)$ & $24(1)$ \\
\hline $\mathrm{C}\left(11^{\prime}\right)$ & $7126(2)$ & $3600(8)$ & $6678(1)$ & $23(1)$ \\
\hline$C\left(12^{\prime}\right)$ & $7959(2)$ & 2835(8) & $6506(1)$ & $24(1)$ \\
\hline$C\left(13^{\prime}\right)$ & $8323(2)$ & $5380(7)$ & $6615(1)$ & $20(1)$ \\
\hline$C\left(14^{\prime}\right)$ & $8065(2)$ & $6397(8)$ & $6197(1)$ & $25(1)$ \\
\hline$C\left(15^{\prime}\right)$ & $7718(2)$ & $3830(8)$ & $6089(1)$ & $25(1)$ \\
\hline$C\left(16^{\prime}\right)$ & 9111(2) & $5711(7)$ & $6819(1)$ & $19(1)$ \\
\hline$C\left(17^{\prime}\right)$ & $9603(2)$ & $7724(8)$ & $6739(1)$ & $23(1)$ \\
\hline$C\left(18^{\prime}\right)$ & $10325(2)$ & $8016(9)$ & $6937(1)$ & $25(1)$ \\
\hline$C\left(19^{\prime}\right)$ & $10579(2)$ & $6329(8)$ & $7216(1)$ & $25(1)$ \\
\hline$C\left(20^{\prime}\right)$ & $10103(3)$ & $4311(8)$ & 7293(1) & $24(1)$ \\
\hline$C\left(21^{\prime}\right)$ & $9375(2)$ & $4005(8)$ & $7097(1)$ & $22(1)$ \\
\hline $\mathrm{F}(1)$ & 7371(3) & $341(7)$ & $5242(1)$ & $72(1)$ \\
\hline $\mathrm{F}(2)$ & $6865(2)$ & $3564(7)$ & $4980(1)$ & $59(1)$ \\
\hline $\mathrm{F}(3)$ & $8089(2)$ & 2696(6) & $4886(1)$ & $52(1)$ \\
\hline $\mathrm{C}(1)$ & $7359(3)$ & $1715(10)$ & $4913(2)$ & $36(1)$ \\
\hline$C(2)$ & $7142(3)$ & $259(9)$ & $4552(1)$ & $27(1)$ \\
\hline$C(3)$ & $6440(2)$ & $718(8)$ & $4341(1)$ & $25(1)$ \\
\hline $\mathrm{C}(4)$ & $6236(2)$ & $-726(7)$ & 4014(1) & $21(1)$ \\
\hline$C(5)$ & $6749(2)$ & $-2597(9)$ & $3899(1)$ & $25(1)$ \\
\hline$C(6)$ & $7456(3)$ & $-3030(9)$ & 4111(1) & $31(1)$ \\
\hline$C(7)$ & 7652(3) & $-1620(9)$ & $4436(1)$ & $32(1)$ \\
\hline $\mathrm{C}(8)$ & $5461(2)$ & $-306(8)$ & $3805(1)$ & $22(1)$ \\
\hline $\mathrm{C}(9)$ & $5077(2)$ & $2236(8)$ & $3706(1)$ & $22(1)$ \\
\hline$C(10)$ & $4856(2)$ & $1296(7)$ & $3278(1)$ & $21(1)$ \\
\hline $\mathrm{C}(11)$ & $5222(2)$ & $-1251(8)$ & $3377(1)$ & $24(1)$ \\
\hline $\mathrm{C}(12)$ & $4389(2)$ & $-2113(8)$ & $3540(1)$ & $25(1)$ \\
\hline$C(13)$ & $4005(2)$ & $407(7)$ & $3436(1)$ & $21(1)$ \\
\hline$C(14)$ & $4242(2)$ & $1367(8)$ & $3864(1)$ & $23(1)$ \\
\hline$C(15)$ & $4609(3)$ & $-1178(8)$ & $3965(1)$ & $26(1)$ \\
\hline$C(16)$ & $3231(2)$ & $694(8)$ & $3221(1)$ & $22(1)$ \\
\hline $\mathrm{C}(17)$ & 2711(3) & $2629(8)$ & $3289(1)$ & $27(1)$ \\
\hline
\end{tabular}




\begin{tabular}{lllll}
$\mathrm{C}(18)$ & $2002(3)$ & $2856(8)$ & $3076(2)$ & $30(1)$ \\
$\mathrm{C}(19)$ & $1798(3)$ & $1126(9)$ & $2789(1)$ & $30(1)$ \\
$\mathrm{C}(20)$ & $2303(3)$ & $-850(9)$ & $2721(1)$ & $28(1)$ \\
$\mathrm{C}(21)$ & $3003(3)$ & $-1064(8)$ & $2938(1)$ & $24(1)$ \\
\hline
\end{tabular}

Table 3. Bond lengths $[\AA]$ and angles $\left[{ }^{\circ}\right]$ for Baran585_a.

\begin{tabular}{|c|c|}
\hline $\mathrm{F}\left(1^{\prime}\right)-\mathrm{C}\left(1^{\prime}\right)$ & $1.329(6)$ \\
\hline $\mathrm{F}\left(2^{\prime}\right)-\mathrm{C}\left(1^{\prime}\right)$ & $1.352(6)$ \\
\hline $\mathrm{F}\left(3^{\prime}\right)-\mathrm{C}\left(1^{\prime}\right)$ & $1.351(6)$ \\
\hline$C\left(1^{\prime}\right)-C\left(2^{\prime}\right)$ & $1.468(6)$ \\
\hline $\mathrm{C}\left(2^{\prime}\right)-\mathrm{C}\left(3^{\prime}\right)$ & $1.378(6)$ \\
\hline$C\left(2^{\prime}\right)-C\left(7^{\prime}\right)$ & $1.403(6)$ \\
\hline $\mathrm{C}\left(3^{\prime}\right)-\mathrm{H}\left(3^{\prime}\right)$ & 0.9500 \\
\hline$C\left(3^{\prime}\right)-C\left(4^{\prime}\right)$ & $1.399(6)$ \\
\hline $\mathrm{C}\left(4^{\prime}\right)-\mathrm{C}\left(5^{\prime}\right)$ & $1.393(6)$ \\
\hline$C\left(4^{\prime}\right)-C\left(8^{\prime}\right)$ & $1.487(5)$ \\
\hline $\mathrm{C}\left(5^{\prime}\right)-\mathrm{H}\left(5^{\prime}\right)$ & 0.9500 \\
\hline$C\left(5^{\prime}\right)-C\left(6^{\prime}\right)$ & $1.387(6)$ \\
\hline $\mathrm{C}\left(6^{\prime}\right)-\mathrm{H}\left(6^{\prime}\right)$ & 0.9500 \\
\hline $\mathrm{C}\left(6^{\prime}\right)-\mathrm{C}\left(7^{\prime}\right)$ & $1.388(6)$ \\
\hline $\mathrm{C}\left(7^{\prime}\right)-\mathrm{H}\left(7^{\prime}\right)$ & 0.9500 \\
\hline $\mathrm{C}\left(8^{\prime}\right)-\mathrm{C}\left(9^{\prime}\right)$ & $1.563(6)$ \\
\hline$C\left(8^{\prime}\right)-C\left(11^{\prime}\right)$ & $1.563(6)$ \\
\hline $\mathrm{C}\left(8^{\prime}\right)-\mathrm{C}\left(15^{\prime}\right)$ & $1.575(6)$ \\
\hline $\mathrm{C}\left(9^{\prime}\right)-\mathrm{H}\left(9^{\prime}\right)$ & 1.0000 \\
\hline $\mathrm{C}\left(9^{\prime}\right)-\mathrm{C}\left(10^{\prime}\right)$ & $1.551(6)$ \\
\hline $\mathrm{C}\left(9^{\prime}\right)-\mathrm{C}\left(14^{\prime}\right)$ & $1.558(6)$ \\
\hline $\mathrm{C}\left(10^{\prime}\right)-\mathrm{H}\left(10^{\prime}\right)$ & 1.0000 \\
\hline $\mathrm{C}\left(10^{\prime}\right)-\mathrm{C}\left(11^{\prime}\right)$ & $1.566(6)$ \\
\hline $\mathrm{C}\left(10^{\prime}\right)-\mathrm{C}\left(13^{\prime}\right)$ & $1.578(6)$ \\
\hline $\mathrm{C}\left(11^{\prime}\right)-\mathrm{H}\left(11^{\prime}\right)$ & 1.0000 \\
\hline $\mathrm{C}\left(11^{\prime}\right)-\mathrm{C}\left(12^{\prime}\right)$ & $1.556(6)$ \\
\hline $\mathrm{C}\left(12^{\prime}\right)-\mathrm{H}\left(12^{\prime}\right)$ & 1.0000 \\
\hline$C\left(12^{\prime}\right)-C\left(13^{\prime}\right)$ & $1.564(6)$ \\
\hline $\mathrm{C}\left(12^{\prime}\right)-\mathrm{C}\left(15^{\prime}\right)$ & $1.552(6)$ \\
\hline
\end{tabular}




\begin{tabular}{|c|c|}
\hline C(13')-C(14') & $1.566(6)$ \\
\hline $\mathrm{C}\left(13^{\prime}\right)-\mathrm{C}\left(16^{\prime}\right)$ & $1.490(5)$ \\
\hline $\mathrm{C}\left(14^{\prime}\right)-\mathrm{H}\left(14^{\prime}\right)$ & 1.0000 \\
\hline$C\left(14^{\prime}\right)-C\left(15^{\prime}\right)$ & $1.563(6)$ \\
\hline $\mathrm{C}\left(15^{\prime}\right)-\mathrm{H}\left(15^{\prime}\right)$ & 1.0000 \\
\hline $\mathrm{C}\left(16^{\prime}\right)-\mathrm{C}\left(17^{\prime}\right)$ & $1.399(6)$ \\
\hline $\mathrm{C}\left(16^{\prime}\right)-\mathrm{C}\left(21^{\prime}\right)$ & $1.391(6)$ \\
\hline $\mathrm{C}\left(17^{\prime}\right)-\mathrm{H}\left(17^{\prime}\right)$ & 0.9500 \\
\hline $\mathrm{C}\left(17^{\prime}\right)-\mathrm{C}\left(18^{\prime}\right)$ & $1.381(6)$ \\
\hline $\mathrm{C}\left(18^{\prime}\right)-\mathrm{H}\left(18^{\prime}\right)$ & 0.9500 \\
\hline $\mathrm{C}\left(18^{\prime}\right)-\mathrm{C}\left(19^{\prime}\right)$ & $1.380(6)$ \\
\hline C(19')-H(19') & 0.9500 \\
\hline $\mathrm{C}\left(19^{\prime}\right)-\mathrm{C}\left(20^{\prime}\right)$ & $1.385(6)$ \\
\hline $\mathrm{C}\left(20^{\prime}\right)-\mathrm{H}\left(20^{\prime}\right)$ & 0.9500 \\
\hline $\mathrm{C}\left(20^{\prime}\right)-\mathrm{C}\left(21^{\prime}\right)$ & $1.388(6)$ \\
\hline $\mathrm{C}\left(21^{\prime}\right)-\mathrm{H}\left(21^{\prime}\right)$ & 0.9500 \\
\hline $\mathrm{F}(1)-\mathrm{C}(1)$ & $1.335(6)$ \\
\hline $\mathrm{F}(2)-\mathrm{C}(1)$ & $1.323(6)$ \\
\hline $\mathrm{F}(3)-\mathrm{C}(1)$ & $1.331(6)$ \\
\hline$C(1)-C(2)$ & $1.491(6)$ \\
\hline$C(2)-C(3)$ & $1.390(6)$ \\
\hline$C(2)-C(7)$ & $1.389(7)$ \\
\hline $\mathrm{C}(3)-\mathrm{H}(3)$ & 0.9500 \\
\hline$C(3)-C(4)$ & $1.392(6)$ \\
\hline$C(4)-C(5)$ & $1.389(6)$ \\
\hline$C(4)-C(8)$ & $1.486(5)$ \\
\hline $\mathrm{C}(5)-\mathrm{H}(5)$ & 0.9500 \\
\hline$C(5)-C(6)$ & $1.395(6)$ \\
\hline $\mathrm{C}(6)-\mathrm{H}(6)$ & 0.9500 \\
\hline$C(6)-C(7)$ & $1.373(6)$ \\
\hline $\mathrm{C}(7)-\mathrm{H}(7)$ & 0.9500 \\
\hline $\mathrm{C}(8)-\mathrm{C}(9)$ & $1.568(6)$ \\
\hline $\mathrm{C}(8)-\mathrm{C}(11)$ & $1.573(5)$ \\
\hline $\mathrm{C}(8)-\mathrm{C}(15)$ & $1.590(6)$ \\
\hline $\mathrm{C}(9)-\mathrm{H}(9)$ & 1.0000 \\
\hline C(9)-C(10) & $1.567(5)$ \\
\hline $\mathrm{C}(9)-\mathrm{C}(14)$ & $1.563(6)$ \\
\hline C(10)-H(10) & 1.0000 \\
\hline
\end{tabular}




\begin{tabular}{|c|c|}
\hline $\mathrm{C}(10)-\mathrm{C}(11)$ & $1.559(6)$ \\
\hline$C(10)-C(13)$ & $1.588(6)$ \\
\hline $\mathrm{C}(11)-\mathrm{H}(11)$ & 1.0000 \\
\hline $\mathrm{C}(11)-\mathrm{C}(12)$ & $1.563(6)$ \\
\hline $\mathrm{C}(12)-\mathrm{H}(12)$ & 1.0000 \\
\hline$C(12)-C(13)$ & $1.561(6)$ \\
\hline$C(12)-C(15)$ & $1.555(6)$ \\
\hline$C(13)-C(14)$ & $1.579(5)$ \\
\hline$C(13)-C(16)$ & $1.483(5)$ \\
\hline $\mathrm{C}(14)-\mathrm{H}(14)$ & 1.0000 \\
\hline C(14)-C(15) & $1.559(6)$ \\
\hline C(15)-H(15) & 1.0000 \\
\hline$C(16)-C(17)$ & $1.389(6)$ \\
\hline$C(16)-C(21)$ & $1.403(6)$ \\
\hline $\mathrm{C}(17)-\mathrm{H}(17)$ & 0.9500 \\
\hline C(17)-C(18) & $1.383(6)$ \\
\hline $\mathrm{C}(18)-\mathrm{H}(18)$ & 0.9500 \\
\hline C(18)-C(19) & $1.393(7)$ \\
\hline C(19)-H(19) & 0.9500 \\
\hline$C(19)-C(20)$ & $1.389(7)$ \\
\hline $\mathrm{C}(20)-\mathrm{H}(20)$ & 0.9500 \\
\hline$C(20)-C(21)$ & $1.376(6)$ \\
\hline $\mathrm{C}(21)-\mathrm{H}(21)$ & 0.9500 \\
\hline $\mathrm{F}\left(1^{\prime}\right)-\mathrm{C}\left(1^{\prime}\right)-\mathrm{F}\left(2^{\prime}\right)$ & $107.0(4)$ \\
\hline $\mathrm{F}\left(1^{\prime}\right)-\mathrm{C}\left(1^{\prime}\right)-\mathrm{F}\left(3^{\prime}\right)$ & $105.1(4)$ \\
\hline $\mathrm{F}\left(1^{\prime}\right)-\mathrm{C}\left(1^{\prime}\right)-\mathrm{C}\left(2^{\prime}\right)$ & $113.5(4)$ \\
\hline $\mathrm{F}\left(2^{\prime}\right)-\mathrm{C}\left(1^{\prime}\right)-\mathrm{C}\left(2^{\prime}\right)$ & $113.2(4)$ \\
\hline $\mathrm{F}\left(3^{\prime}\right)-\mathrm{C}\left(1^{\prime}\right)-\mathrm{F}\left(2^{\prime}\right)$ & $104.2(4)$ \\
\hline $\mathrm{F}\left(3^{\prime}\right)-\mathrm{C}\left(1^{\prime}\right)-\mathrm{C}\left(2^{\prime}\right)$ & $113.0(4)$ \\
\hline $\mathrm{C}\left(3^{\prime}\right)-\mathrm{C}\left(2^{\prime}\right)-\mathrm{C}\left(1^{\prime}\right)$ & $122.4(4)$ \\
\hline$C\left(3^{\prime}\right)-C\left(2^{\prime}\right)-C\left(7^{\prime}\right)$ & $120.0(4)$ \\
\hline $\mathrm{C}\left(7^{\prime}\right)-\mathrm{C}\left(2^{\prime}\right)-\mathrm{C}\left(1^{\prime}\right)$ & $117.5(4)$ \\
\hline $\mathrm{C}\left(2^{\prime}\right)-\mathrm{C}\left(3^{\prime}\right)-\mathrm{H}\left(3^{\prime}\right)$ & 119.3 \\
\hline $\mathrm{C}\left(2^{\prime}\right)-\mathrm{C}\left(3^{\prime}\right)-\mathrm{C}\left(4^{\prime}\right)$ & $121.4(4)$ \\
\hline $\mathrm{C}\left(4^{\prime}\right)-\mathrm{C}\left(3^{\prime}\right)-\mathrm{H}\left(3^{\prime}\right)$ & 119.3 \\
\hline$C\left(3^{\prime}\right)-C\left(4^{\prime}\right)-C\left(8^{\prime}\right)$ & $120.1(4)$ \\
\hline$C\left(5^{\prime}\right)-C\left(4^{\prime}\right)-C\left(3^{\prime}\right)$ & $118.2(4)$ \\
\hline
\end{tabular}




\begin{tabular}{|c|c|}
\hline $\mathrm{C}\left(5^{\prime}\right)-\mathrm{C}\left(4^{\prime}\right)-\mathrm{C}\left(8^{\prime}\right)$ & $121.7(4)$ \\
\hline $\mathrm{C}\left(4^{\prime}\right)-\mathrm{C}\left(5^{\prime}\right)-\mathrm{H}\left(5^{\prime}\right)$ & 119.6 \\
\hline$C\left(6^{\prime}\right)-C\left(5^{\prime}\right)-C\left(4^{\prime}\right)$ & $120.8(4)$ \\
\hline $\mathrm{C}\left(6^{\prime}\right)-\mathrm{C}\left(5^{\prime}\right)-\mathrm{H}\left(5^{\prime}\right)$ & 119.6 \\
\hline $\mathrm{C}\left(5^{\prime}\right)-\mathrm{C}\left(6^{\prime}\right)-\mathrm{H}\left(6^{\prime}\right)$ & 119.7 \\
\hline $\mathrm{C}\left(5^{\prime}\right)-\mathrm{C}\left(6^{\prime}\right)-\mathrm{C}\left(7^{\prime}\right)$ & $120.6(4)$ \\
\hline $\mathrm{C}\left(7^{\prime}\right)-\mathrm{C}\left(6^{\prime}\right)-\mathrm{H}\left(6^{\prime}\right)$ & 119.7 \\
\hline $\mathrm{C}\left(2^{\prime}\right)-\mathrm{C}\left(7^{\prime}\right)-\mathrm{H}\left(7^{\prime}\right)$ & 120.5 \\
\hline$C\left(6^{\prime}\right)-C\left(7^{\prime}\right)-C\left(2^{\prime}\right)$ & $119.0(4)$ \\
\hline $\mathrm{C}\left(6^{\prime}\right)-\mathrm{C}\left(7^{\prime}\right)-\mathrm{H}\left(7^{\prime}\right)$ & 120.5 \\
\hline $\mathrm{C}\left(4^{\prime}\right)-\mathrm{C}\left(8^{\prime}\right)-\mathrm{C}\left(9^{\prime}\right)$ & $125.2(4)$ \\
\hline $\mathrm{C}\left(4^{\prime}\right)-\mathrm{C}\left(8^{\prime}\right)-\mathrm{C}\left(11^{\prime}\right)$ & $127.4(3)$ \\
\hline $\mathrm{C}\left(4^{\prime}\right)-\mathrm{C}\left(8^{\prime}\right)-\mathrm{C}\left(15^{\prime}\right)$ & $124.1(3)$ \\
\hline $\mathrm{C}\left(9^{\prime}\right)-\mathrm{C}\left(8^{\prime}\right)-\mathrm{C}\left(11^{\prime}\right)$ & $90.1(3)$ \\
\hline $\mathrm{C}\left(9^{\prime}\right)-\mathrm{C}\left(8^{\prime}\right)-\mathrm{C}\left(15^{\prime}\right)$ & $89.3(3)$ \\
\hline $\mathrm{C}\left(11^{\prime}\right)-\mathrm{C}\left(8^{\prime}\right)-\mathrm{C}\left(15^{\prime}\right)$ & $89.3(3)$ \\
\hline $\mathrm{C}\left(8^{\prime}\right)-\mathrm{C}\left(9^{\prime}\right)-\mathrm{H}\left(9^{\prime}\right)$ & 125.0 \\
\hline $\mathrm{C}\left(10^{\prime}\right)-\mathrm{C}\left(9^{\prime}\right)-\mathrm{C}\left(8^{\prime}\right)$ & $90.0(3)$ \\
\hline $\mathrm{C}\left(10^{\prime}\right)-\mathrm{C}\left(9^{\prime}\right)-\mathrm{H}\left(9^{\prime}\right)$ & 125.0 \\
\hline $\mathrm{C}\left(10^{\prime}\right)-\mathrm{C}\left(9^{\prime}\right)-\mathrm{C}\left(14^{\prime}\right)$ & $90.3(3)$ \\
\hline $\mathrm{C}\left(14^{\prime}\right)-\mathrm{C}\left(9^{\prime}\right)-\mathrm{C}\left(8^{\prime}\right)$ & $90.7(3)$ \\
\hline $\mathrm{C}\left(14^{\prime}\right)-\mathrm{C}\left(9^{\prime}\right)-\mathrm{H}\left(9^{\prime}\right)$ & 125.0 \\
\hline $\mathrm{C}\left(9^{\prime}\right)-\mathrm{C}\left(10^{\prime}\right)-\mathrm{H}\left(10^{\prime}\right)$ & 125.1 \\
\hline $\mathrm{C}\left(9^{\prime}\right)-\mathrm{C}\left(10^{\prime}\right)-\mathrm{C}\left(11^{\prime}\right)$ & $90.4(3)$ \\
\hline$C\left(9^{\prime}\right)-C\left(10^{\prime}\right)-C\left(13^{\prime}\right)$ & $90.3(3)$ \\
\hline $\mathrm{C}\left(11^{\prime}\right)-\mathrm{C}\left(10^{\prime}\right)-\mathrm{H}\left(10^{\prime}\right)$ & 125.1 \\
\hline$C\left(11^{\prime}\right)-C\left(10^{\prime}\right)-C\left(13^{\prime}\right)$ & $90.2(3)$ \\
\hline $\mathrm{C}\left(13^{\prime}\right)-\mathrm{C}\left(10^{\prime}\right)-\mathrm{H}\left(10^{\prime}\right)$ & 125.1 \\
\hline $\mathrm{C}\left(8^{\prime}\right)-\mathrm{C}\left(11^{\prime}\right)-\mathrm{C}\left(10^{\prime}\right)$ & $89.5(3)$ \\
\hline $\mathrm{C}\left(8^{\prime}\right)-\mathrm{C}\left(11^{\prime}\right)-\mathrm{H}\left(11^{\prime}\right)$ & 125.4 \\
\hline $\mathrm{C}\left(10^{\prime}\right)-\mathrm{C}\left(11^{\prime}\right)-\mathrm{H}\left(11^{\prime}\right)$ & 125.4 \\
\hline $\mathrm{C}\left(12^{\prime}\right)-\mathrm{C}\left(11^{\prime}\right)-\mathrm{C}\left(8^{\prime}\right)$ & $90.3(3)$ \\
\hline $\mathrm{C}\left(12^{\prime}\right)-\mathrm{C}\left(11^{\prime}\right)-\mathrm{C}\left(10^{\prime}\right)$ & $89.8(3)$ \\
\hline $\mathrm{C}\left(12^{\prime}\right)-\mathrm{C}\left(11^{\prime}\right)-\mathrm{H}\left(11^{\prime}\right)$ & 125.4 \\
\hline $\mathrm{C}\left(11^{\prime}\right)-\mathrm{C}\left(12^{\prime}\right)-\mathrm{H}\left(12^{\prime}\right)$ & 125.0 \\
\hline $\mathrm{C}\left(11^{\prime}\right)-\mathrm{C}\left(12^{\prime}\right)-\mathrm{C}\left(13^{\prime}\right)$ & $91.0(3)$ \\
\hline $\mathrm{C}\left(13^{\prime}\right)-\mathrm{C}\left(12^{\prime}\right)-\mathrm{H}\left(12^{\prime}\right)$ & 125.0 \\
\hline $\mathrm{C}\left(15^{\prime}\right)-\mathrm{C}\left(12^{\prime}\right)-\mathrm{C}\left(11^{\prime}\right)$ & $90.4(3)$ \\
\hline
\end{tabular}




\begin{tabular}{|c|c|}
\hline $\mathrm{C}\left(15^{\prime}\right)-\mathrm{C}\left(12^{\prime}\right)-\mathrm{H}\left(12^{\prime}\right)$ & 125.0 \\
\hline $\mathrm{C}\left(15^{\prime}\right)-\mathrm{C}\left(12^{\prime}\right)-\mathrm{C}\left(13^{\prime}\right)$ & $89.8(3)$ \\
\hline $\mathrm{C}\left(12^{\prime}\right)-\mathrm{C}\left(13^{\prime}\right)-\mathrm{C}\left(10^{\prime}\right)$ & $89.0(3)$ \\
\hline $\mathrm{C}\left(12^{\prime}\right)-\mathrm{C}\left(13^{\prime}\right)-\mathrm{C}\left(14^{\prime}\right)$ & $90.2(3)$ \\
\hline $\mathrm{C}\left(14^{\prime}\right)-\mathrm{C}\left(13^{\prime}\right)-\mathrm{C}\left(10^{\prime}\right)$ & $89.0(3)$ \\
\hline $\mathrm{C}\left(16^{\prime}\right)-\mathrm{C}\left(13^{\prime}\right)-\mathrm{C}\left(10^{\prime}\right)$ & $125.9(3)$ \\
\hline $\mathrm{C}\left(16^{\prime}\right)-\mathrm{C}\left(13^{\prime}\right)-\mathrm{C}\left(12^{\prime}\right)$ & $123.8(3)$ \\
\hline $\mathrm{C}\left(16^{\prime}\right)-\mathrm{C}\left(13^{\prime}\right)-\mathrm{C}\left(14^{\prime}\right)$ & $127.3(4)$ \\
\hline $\mathrm{C}\left(9^{\prime}\right)-\mathrm{C}\left(14^{\prime}\right)-\mathrm{C}\left(13^{\prime}\right)$ & $90.5(3)$ \\
\hline $\mathrm{C}\left(9^{\prime}\right)-\mathrm{C}\left(14^{\prime}\right)-\mathrm{H}\left(14^{\prime}\right)$ & 125.4 \\
\hline $\mathrm{C}\left(9^{\prime}\right)-\mathrm{C}\left(14^{\prime}\right)-\mathrm{C}\left(15^{\prime}\right)$ & $89.9(3)$ \\
\hline $\mathrm{C}\left(13^{\prime}\right)-\mathrm{C}\left(14^{\prime}\right)-\mathrm{H}\left(14^{\prime}\right)$ & 125.4 \\
\hline $\mathrm{C}\left(15^{\prime}\right)-\mathrm{C}\left(14^{\prime}\right)-\mathrm{C}\left(13^{\prime}\right)$ & $89.3(3)$ \\
\hline $\mathrm{C}\left(15^{\prime}\right)-\mathrm{C}\left(14^{\prime}\right)-\mathrm{H}\left(14^{\prime}\right)$ & 125.4 \\
\hline $\mathrm{C}\left(8^{\prime}\right)-\mathrm{C}\left(15^{\prime}\right)-\mathrm{H}\left(15^{\prime}\right)$ & 125.1 \\
\hline $\mathrm{C}\left(12^{\prime}\right)-\mathrm{C}\left(15^{\prime}\right)-\mathrm{C}\left(8^{\prime}\right)$ & $90.0(3)$ \\
\hline $\mathrm{C}\left(12^{\prime}\right)-\mathrm{C}\left(15^{\prime}\right)-\mathrm{C}\left(14^{\prime}\right)$ & $90.7(3)$ \\
\hline $\mathrm{C}\left(12^{\prime}\right)-\mathrm{C}\left(15^{\prime}\right)-\mathrm{H}\left(15^{\prime}\right)$ & 125.1 \\
\hline $\mathrm{C}\left(14^{\prime}\right)-\mathrm{C}\left(15^{\prime}\right)-\mathrm{C}\left(8^{\prime}\right)$ & $90.1(3)$ \\
\hline $\mathrm{C}\left(14^{\prime}\right)-\mathrm{C}\left(15^{\prime}\right)-\mathrm{H}\left(15^{\prime}\right)$ & 125.1 \\
\hline $\mathrm{C}\left(17^{\prime}\right)-\mathrm{C}\left(16^{\prime}\right)-\mathrm{C}\left(13^{\prime}\right)$ & $121.6(4)$ \\
\hline $\mathrm{C}\left(21^{\prime}\right)-\mathrm{C}\left(16^{\prime}\right)-\mathrm{C}\left(13^{\prime}\right)$ & $120.1(4)$ \\
\hline $\mathrm{C}\left(21^{\prime}\right)-\mathrm{C}\left(16^{\prime}\right)-\mathrm{C}\left(17^{\prime}\right)$ & $118.3(4)$ \\
\hline $\mathrm{C}\left(16^{\prime}\right)-\mathrm{C}\left(17^{\prime}\right)-\mathrm{H}\left(17^{\prime}\right)$ & 119.7 \\
\hline $\mathrm{C}\left(18^{\prime}\right)-\mathrm{C}\left(17^{\prime}\right)-\mathrm{C}\left(16^{\prime}\right)$ & $120.5(4)$ \\
\hline $\mathrm{C}\left(18^{\prime}\right)-\mathrm{C}\left(17^{\prime}\right)-\mathrm{H}\left(17^{\prime}\right)$ & 119.7 \\
\hline $\mathrm{C}\left(17^{\prime}\right)-\mathrm{C}\left(18^{\prime}\right)-\mathrm{H}\left(18^{\prime}\right)$ & 119.6 \\
\hline $\mathrm{C}\left(19^{\prime}\right)-\mathrm{C}\left(18^{\prime}\right)-\mathrm{C}\left(17^{\prime}\right)$ & $120.9(4)$ \\
\hline $\mathrm{C}\left(19^{\prime}\right)-\mathrm{C}\left(18^{\prime}\right)-\mathrm{H}\left(18^{\prime}\right)$ & 119.6 \\
\hline $\mathrm{C}\left(18^{\prime}\right)-\mathrm{C}\left(19^{\prime}\right)-\mathrm{H}\left(19^{\prime}\right)$ & 120.4 \\
\hline $\mathrm{C}\left(18^{\prime}\right)-\mathrm{C}\left(19^{\prime}\right)-\mathrm{C}\left(20^{\prime}\right)$ & $119.1(4)$ \\
\hline $\mathrm{C}\left(20^{\prime}\right)-\mathrm{C}\left(19^{\prime}\right)-\mathrm{H}\left(19^{\prime}\right)$ & 120.4 \\
\hline $\mathrm{C}\left(19^{\prime}\right)-\mathrm{C}\left(20^{\prime}\right)-\mathrm{H}\left(20^{\prime}\right)$ & 119.8 \\
\hline $\mathrm{C}\left(19^{\prime}\right)-\mathrm{C}\left(20^{\prime}\right)-\mathrm{C}\left(21^{\prime}\right)$ & $120.5(4)$ \\
\hline $\mathrm{C}\left(21^{\prime}\right)-\mathrm{C}\left(20^{\prime}\right)-\mathrm{H}\left(20^{\prime}\right)$ & 119.8 \\
\hline $\mathrm{C}\left(16^{\prime}\right)-\mathrm{C}\left(21^{\prime}\right)-\mathrm{H}\left(21^{\prime}\right)$ & 119.7 \\
\hline $\mathrm{C}\left(20^{\prime}\right)-\mathrm{C}\left(21^{\prime}\right)-\mathrm{C}\left(16^{\prime}\right)$ & $120.7(4)$ \\
\hline $\mathrm{C}\left(20^{\prime}\right)-\mathrm{C}\left(21^{\prime}\right)-\mathrm{H}\left(21^{\prime}\right)$ & 119.7 \\
\hline
\end{tabular}




\begin{tabular}{|c|c|}
\hline $\mathrm{F}(1)-\mathrm{C}(1)-\mathrm{C}(2)$ & $111.7(5)$ \\
\hline $\mathrm{F}(2)-\mathrm{C}(1)-\mathrm{F}(1)$ & $107.5(4)$ \\
\hline $\mathrm{F}(2)-\mathrm{C}(1)-\mathrm{F}(3)$ & $105.5(5)$ \\
\hline $\mathrm{F}(2)-\mathrm{C}(1)-\mathrm{C}(2)$ & $113.5(4)$ \\
\hline $\mathrm{F}(3)-\mathrm{C}(1)-\mathrm{F}(1)$ & $105.6(4)$ \\
\hline $\mathrm{F}(3)-\mathrm{C}(1)-\mathrm{C}(2)$ & $112.5(4)$ \\
\hline$C(3)-C(2)-C(1)$ & $121.2(4)$ \\
\hline$C(7)-C(2)-C(1)$ & $118.4(4)$ \\
\hline $\mathrm{C}(7)-\mathrm{C}(2)-\mathrm{C}(3)$ & $120.3(4)$ \\
\hline $\mathrm{C}(2)-\mathrm{C}(3)-\mathrm{H}(3)$ & 119.9 \\
\hline$C(2)-C(3)-C(4)$ & $120.1(4)$ \\
\hline $\mathrm{C}(4)-\mathrm{C}(3)-\mathrm{H}(3)$ & 119.9 \\
\hline $\mathrm{C}(3)-\mathrm{C}(4)-\mathrm{C}(8)$ & $119.6(4)$ \\
\hline$C(5)-C(4)-C(3)$ & $119.2(4)$ \\
\hline$C(5)-C(4)-C(8)$ & 121.1(4) \\
\hline $\mathrm{C}(4)-\mathrm{C}(5)-\mathrm{H}(5)$ & 119.9 \\
\hline$C(4)-C(5)-C(6)$ & $120.1(4)$ \\
\hline $\mathrm{C}(6)-\mathrm{C}(5)-\mathrm{H}(5)$ & 119.9 \\
\hline $\mathrm{C}(5)-\mathrm{C}(6)-\mathrm{H}(6)$ & 119.7 \\
\hline$C(7)-C(6)-C(5)$ & $120.5(4)$ \\
\hline $\mathrm{C}(7)-\mathrm{C}(6)-\mathrm{H}(6)$ & 119.7 \\
\hline $\mathrm{C}(2)-\mathrm{C}(7)-\mathrm{H}(7)$ & 120.2 \\
\hline$C(6)-C(7)-C(2)$ & $119.6(4)$ \\
\hline $\mathrm{C}(6)-\mathrm{C}(7)-\mathrm{H}(7)$ & 120.2 \\
\hline $\mathrm{C}(4)-\mathrm{C}(8)-\mathrm{C}(9)$ & $126.2(3)$ \\
\hline $\mathrm{C}(4)-\mathrm{C}(8)-\mathrm{C}(11)$ & $126.8(3)$ \\
\hline $\mathrm{C}(4)-\mathrm{C}(8)-\mathrm{C}(15)$ & $124.6(3)$ \\
\hline $\mathrm{C}(9)-\mathrm{C}(8)-\mathrm{C}(11)$ & $89.9(3)$ \\
\hline $\mathrm{C}(9)-\mathrm{C}(8)-\mathrm{C}(15)$ & $88.6(3)$ \\
\hline$C(11)-C(8)-C(15)$ & $89.0(3)$ \\
\hline $\mathrm{C}(8)-\mathrm{C}(9)-\mathrm{H}(9)$ & 125.0 \\
\hline $\mathrm{C}(10)-\mathrm{C}(9)-\mathrm{C}(8)$ & $89.8(3)$ \\
\hline $\mathrm{C}(10)-\mathrm{C}(9)-\mathrm{H}(9)$ & 125.0 \\
\hline $\mathrm{C}(14)-\mathrm{C}(9)-\mathrm{C}(8)$ & $91.1(3)$ \\
\hline $\mathrm{C}(14)-\mathrm{C}(9)-\mathrm{H}(9)$ & 125.0 \\
\hline $\mathrm{C}(14)-\mathrm{C}(9)-\mathrm{C}(10)$ & $90.1(3)$ \\
\hline $\mathrm{C}(9)-\mathrm{C}(10)-\mathrm{H}(10)$ & 125.1 \\
\hline $\mathrm{C}(9)-\mathrm{C}(10)-\mathrm{C}(13)$ & $90.3(3)$ \\
\hline
\end{tabular}




\begin{tabular}{|c|c|}
\hline $\mathrm{C}(11)-\mathrm{C}(10)-\mathrm{C}(9)$ & $90.4(3)$ \\
\hline $\mathrm{C}(11)-\mathrm{C}(10)-\mathrm{H}(10)$ & 125.1 \\
\hline $\mathrm{C}(11)-\mathrm{C}(10)-\mathrm{C}(13)$ & $90.1(3)$ \\
\hline $\mathrm{C}(13)-\mathrm{C}(10)-\mathrm{H}(10)$ & 125.1 \\
\hline $\mathrm{C}(8)-\mathrm{C}(11)-\mathrm{H}(11)$ & 125.2 \\
\hline $\mathrm{C}(10)-\mathrm{C}(11)-\mathrm{C}(8)$ & $89.9(3)$ \\
\hline $\mathrm{C}(10)-\mathrm{C}(11)-\mathrm{H}(11)$ & 125.2 \\
\hline $\mathrm{C}(10)-\mathrm{C}(11)-\mathrm{C}(12)$ & $90.0(3)$ \\
\hline $\mathrm{C}(12)-\mathrm{C}(11)-\mathrm{C}(8)$ & $90.4(3)$ \\
\hline $\mathrm{C}(12)-\mathrm{C}(11)-\mathrm{H}(11)$ & 125.2 \\
\hline $\mathrm{C}(11)-\mathrm{C}(12)-\mathrm{H}(12)$ & 124.8 \\
\hline $\mathrm{C}(13)-\mathrm{C}(12)-\mathrm{C}(11)$ & $91.0(3)$ \\
\hline $\mathrm{C}(13)-\mathrm{C}(12)-\mathrm{H}(12)$ & 124.8 \\
\hline $\mathrm{C}(15)-\mathrm{C}(12)-\mathrm{C}(11)$ & $90.6(3)$ \\
\hline $\mathrm{C}(15)-\mathrm{C}(12)-\mathrm{H}(12)$ & 124.8 \\
\hline $\mathrm{C}(15)-\mathrm{C}(12)-\mathrm{C}(13)$ & $90.5(3)$ \\
\hline $\mathrm{C}(12)-\mathrm{C}(13)-\mathrm{C}(10)$ & $88.9(3)$ \\
\hline $\mathrm{C}(12)-\mathrm{C}(13)-\mathrm{C}(14)$ & $89.4(3)$ \\
\hline$C(14)-C(13)-C(10)$ & $88.8(3)$ \\
\hline$C(16)-C(13)-C(10)$ & $125.4(3)$ \\
\hline$C(16)-C(13)-C(12)$ & $123.9(3)$ \\
\hline$C(16)-C(13)-C(14)$ & $128.5(3)$ \\
\hline $\mathrm{C}(9)-\mathrm{C}(14)-\mathrm{C}(13)$ & $90.8(3)$ \\
\hline $\mathrm{C}(9)-\mathrm{C}(14)-\mathrm{H}(14)$ & 125.2 \\
\hline $\mathrm{C}(13)-\mathrm{C}(14)-\mathrm{H}(14)$ & 125.2 \\
\hline $\mathrm{C}(15)-\mathrm{C}(14)-\mathrm{C}(9)$ & $89.9(3)$ \\
\hline$C(15)-C(14)-C(13)$ & $89.7(3)$ \\
\hline $\mathrm{C}(15)-\mathrm{C}(14)-\mathrm{H}(14)$ & 125.2 \\
\hline $\mathrm{C}(8)-\mathrm{C}(15)-\mathrm{H}(15)$ & 125.1 \\
\hline $\mathrm{C}(12)-\mathrm{C}(15)-\mathrm{C}(8)$ & $90.0(3)$ \\
\hline $\mathrm{C}(12)-\mathrm{C}(15)-\mathrm{C}(14)$ & $90.3(3)$ \\
\hline $\mathrm{C}(12)-\mathrm{C}(15)-\mathrm{H}(15)$ & 125.1 \\
\hline $\mathrm{C}(14)-\mathrm{C}(15)-\mathrm{C}(8)$ & $90.4(3)$ \\
\hline $\mathrm{C}(14)-\mathrm{C}(15)-\mathrm{H}(15)$ & 125.1 \\
\hline$C(17)-C(16)-C(13)$ & $122.8(4)$ \\
\hline$C(17)-C(16)-C(21)$ & $117.8(4)$ \\
\hline $\mathrm{C}(21)-\mathrm{C}(16)-\mathrm{C}(13)$ & $119.3(4)$ \\
\hline $\mathrm{C}(16)-\mathrm{C}(17)-\mathrm{H}(17)$ & 119.5 \\
\hline
\end{tabular}




$\begin{array}{ll}\mathrm{C}(18)-\mathrm{C}(17)-\mathrm{C}(16) & 121.0(4) \\ \mathrm{C}(18)-\mathrm{C}(17)-\mathrm{H}(17) & 119.5 \\ \mathrm{C}(17)-\mathrm{C}(18)-\mathrm{H}(18) & 119.9 \\ \mathrm{C}(17)-\mathrm{C}(18)-\mathrm{C}(19) & 120.2(4) \\ \mathrm{C}(19)-\mathrm{C}(18)-\mathrm{H}(18) & 119.9 \\ \mathrm{C}(18)-\mathrm{C}(19)-\mathrm{H}(19) & 120.1 \\ \mathrm{C}(20)-\mathrm{C}(19)-\mathrm{C}(18) & 119.7(4) \\ \mathrm{C}(20)-\mathrm{C}(19)-\mathrm{H}(19) & 120.1 \\ \mathrm{C}(19)-\mathrm{C}(20)-\mathrm{H}(20) & 120.3 \\ \mathrm{C}(21)-\mathrm{C}(20)-\mathrm{C}(19) & 119.5(4) \\ \mathrm{C}(21)-\mathrm{C}(20)-\mathrm{H}(20) & 120.3 \\ \mathrm{C}(16)-\mathrm{C}(21)-\mathrm{H}(21) & 119.1 \\ \mathrm{C}(20)-\mathrm{C}(21)-\mathrm{C}(16) & 121.8(4) \\ \mathrm{C}(20)-\mathrm{C}(21)-\mathrm{H}(21) & 119.1\end{array}$


Table 4. Anisotropic displacement parameters $\left(\AA^{2} \times 10^{3}\right)$ for Baran585_a. The anisotropic displacement factor exponent takes the form: $-2 \pi^{2}\left[h^{2} a^{* 2} U^{11}+\ldots+2 h k a^{*} b^{*} U^{12}\right]$

\begin{tabular}{|c|c|c|c|c|c|c|}
\hline & $\mathrm{U}^{11}$ & $\mathrm{U}^{22}$ & $\mathrm{U}^{33}$ & $\mathrm{U}^{23}$ & $\mathrm{U}^{13}$ & $\mathrm{U}^{12}$ \\
\hline $\mathrm{F}\left(1^{\prime}\right)$ & $110(3)$ & $74(2)$ & $22(2)$ & $5(2)$ & $-19(2)$ & $15(2)$ \\
\hline$F\left(2^{\prime}\right)$ & $48(2)$ & $73(2)$ & $50(2)$ & $35(2)$ & $-13(1)$ & $-16(2)$ \\
\hline $\mathrm{F}\left(3^{\prime}\right)$ & $38(2)$ & $61(2)$ & $62(2)$ & $23(2)$ & $-19(2)$ & $10(2)$ \\
\hline$C\left(1^{\prime}\right)$ & $37(3)$ & $45(3)$ & $31(3)$ & $11(2)$ & $-11(2)$ & $1(2)$ \\
\hline$C\left(2^{\prime}\right)$ & $19(2)$ & $37(3)$ & $18(2)$ & $3(2)$ & $-5(2)$ & $-1(2)$ \\
\hline$C\left(3^{\prime}\right)$ & $21(2)$ & $28(2)$ & $20(2)$ & 1(2) & $-1(2)$ & $-1(2)$ \\
\hline$C\left(4^{\prime}\right)$ & $14(2)$ & $26(2)$ & $17(2)$ & $-2(2)$ & $-1(1)$ & $1(2)$ \\
\hline$C\left(5^{\prime}\right)$ & $23(2)$ & $26(2)$ & $16(2)$ & $2(2)$ & $-4(2)$ & $-3(2)$ \\
\hline$C\left(6^{\prime}\right)$ & $21(2)$ & $38(2)$ & $23(2)$ & $4(2)$ & $-4(2)$ & $-7(2)$ \\
\hline$C\left(7^{\prime}\right)$ & $19(2)$ & $46(3)$ & $21(2)$ & $1(2)$ & $-8(2)$ & $-5(2)$ \\
\hline $\mathrm{C}\left(8^{\prime}\right)$ & $14(2)$ & $22(2)$ & $24(2)$ & $-4(2)$ & $-3(2)$ & $0(2)$ \\
\hline $\mathrm{C}\left(9^{\prime}\right)$ & $18(2)$ & $20(2)$ & $34(2)$ & $-3(2)$ & $-6(2)$ & $0(2)$ \\
\hline $\mathrm{C}\left(10^{\prime}\right)$ & $16(2)$ & $34(2)$ & $23(2)$ & $-9(2)$ & $-4(2)$ & $2(2)$ \\
\hline$C\left(11^{\prime}\right)$ & $14(2)$ & $32(2)$ & $22(2)$ & $2(2)$ & $-6(2)$ & $-4(2)$ \\
\hline $\mathrm{C}\left(12^{\prime}\right)$ & $19(2)$ & $25(2)$ & $29(2)$ & $-4(2)$ & $-4(2)$ & $-1(2)$ \\
\hline$C\left(13^{\prime}\right)$ & $14(2)$ & $22(2)$ & $24(2)$ & $-4(2)$ & $-2(2)$ & $1(2)$ \\
\hline$C\left(14^{\prime}\right)$ & $20(2)$ & $29(2)$ & $26(2)$ & $0(2)$ & $-3(2)$ & $-1(2)$ \\
\hline$C\left(15^{\prime}\right)$ & $16(2)$ & $36(2)$ & $23(2)$ & $-9(2)$ & $-2(2)$ & $-1(2)$ \\
\hline$C\left(16^{\prime}\right)$ & $15(2)$ & $23(2)$ & $18(2)$ & $-6(2)$ & $-1(1)$ & $2(2)$ \\
\hline$C\left(17^{\prime}\right)$ & $18(2)$ & $23(2)$ & $27(2)$ & $-1(2)$ & $-4(2)$ & $3(2)$ \\
\hline $\mathrm{C}\left(18^{\prime}\right)$ & $20(2)$ & $29(2)$ & $26(2)$ & $0(2)$ & $-2(2)$ & $-1(2)$ \\
\hline $\mathrm{C}\left(19^{\prime}\right)$ & $16(2)$ & 41(3) & $17(2)$ & $-7(2)$ & $-2(1)$ & $-4(2)$ \\
\hline $\mathrm{C}\left(20^{\prime}\right)$ & $25(2)$ & $34(2)$ & $14(2)$ & $2(2)$ & $-1(2)$ & $0(2)$ \\
\hline$C\left(21^{\prime}\right)$ & $18(2)$ & $28(2)$ & $20(2)$ & $-4(2)$ & $1(2)$ & $-2(2)$ \\
\hline $\mathrm{F}(1)$ & $113(3)$ & $81(3)$ & $21(2)$ & $-1(2)$ & $-22(2)$ & $-16(2)$ \\
\hline$F(2)$ & $50(2)$ & $79(3)$ & $49(2)$ & $-44(2)$ & $-16(1)$ & $17(2)$ \\
\hline $\mathrm{F}(3)$ & $38(2)$ & $58(2)$ & $61(2)$ & $-20(2)$ & $-21(2)$ & $-1(2)$ \\
\hline $\mathrm{C}(1)$ & $30(3)$ & $51(3)$ & $28(2)$ & $-12(3)$ & $-12(2)$ & $6(2)$ \\
\hline $\mathrm{C}(2)$ & $23(2)$ & $41(3)$ & $17(2)$ & $1(2)$ & $-2(2)$ & $-2(2)$ \\
\hline$C(3)$ & $21(2)$ & $34(2)$ & $20(2)$ & $-3(2)$ & $-2(2)$ & $3(2)$ \\
\hline $\mathrm{C}(4)$ & $20(2)$ & $29(2)$ & $14(2)$ & $2(2)$ & $0(1)$ & $1(2)$ \\
\hline $\mathrm{C}(5)$ & $21(2)$ & $33(2)$ & $21(2)$ & $-6(2)$ & $-3(2)$ & $-1(2)$ \\
\hline $\mathrm{C}(6)$ & $23(2)$ & $39(3)$ & $31(2)$ & $-4(2)$ & $-2(2)$ & $10(2)$ \\
\hline
\end{tabular}




\begin{tabular}{lllllll}
$\mathrm{C}(7)$ & $19(2)$ & $52(3)$ & $24(2)$ & $0(2)$ & $-8(2)$ & $9(2)$ \\
$\mathrm{C}(8)$ & $21(2)$ & $27(2)$ & $17(2)$ & $-4(2)$ & $-2(2)$ & $0(2)$ \\
$\mathrm{C}(9)$ & $21(2)$ & $24(2)$ & $21(2)$ & $-1(2)$ & $-4(2)$ & $-4(2)$ \\
$\mathrm{C}(10)$ & $19(2)$ & $25(2)$ & $20(2)$ & $3(2)$ & $0(2)$ & $0(2)$ \\
$\mathrm{C}(11)$ & $19(2)$ & $28(2)$ & $23(2)$ & $-3(2)$ & $-4(2)$ & $2(2)$ \\
$\mathrm{C}(12)$ & $22(2)$ & $22(2)$ & $30(3)$ & $5(2)$ & $-11(2)$ & $-1(2)$ \\
$\mathrm{C}(13)$ & $18(2)$ & $23(2)$ & $23(2)$ & $3(2)$ & $0(2)$ & $-1(2)$ \\
$\mathrm{C}(14)$ & $18(2)$ & $34(2)$ & $18(2)$ & $-2(2)$ & $-1(2)$ & $2(2)$ \\
$\mathrm{C}(15)$ & $20(2)$ & $36(2)$ & $21(2)$ & $7(2)$ & $-4(2)$ & $-1(2)$ \\
$\mathrm{C}(16)$ & $17(2)$ & $28(2)$ & $19(2)$ & $7(2)$ & $0(2)$ & $-4(2)$ \\
$\mathrm{C}(17)$ & $25(2)$ & $27(2)$ & $28(2)$ & $0(2)$ & $-6(2)$ & $1(2)$ \\
$\mathrm{C}(18)$ & $26(2)$ & $29(2)$ & $36(3)$ & $8(2)$ & $-3(2)$ & $5(2)$ \\
$\mathrm{C}(19)$ & $19(2)$ & $41(3)$ & $30(2)$ & $12(2)$ & $-7(2)$ & $-2(2)$ \\
$\mathrm{C}(20)$ & $26(2)$ & $38(2)$ & $19(2)$ & $0(2)$ & $-2(2)$ & $0(2)$ \\
$\mathrm{C}(21)$ & $21(2)$ & $32(2)$ & $19(2)$ & $2(2)$ & $-1(2)$ & $3(2)$ \\
\hline
\end{tabular}


Table 5. Hydrogen coordinates ( $\left.\mathrm{x} 10^{4}\right)$ and isotropic displacement parameters $\left(\AA^{2} \times 10^{3}\right)$ for Baran585_a.

\begin{tabular}{|c|c|c|c|c|}
\hline & $\mathrm{x}$ & $\mathrm{y}$ & $\mathrm{z}$ & $\mathrm{U}(\mathrm{eq})$ \\
\hline $\mathrm{H}\left(3^{\prime}\right)$ & 6235 & 6970 & 5646 & 28 \\
\hline $\mathrm{H}\left(5^{\prime}\right)$ & 5711 & 1301 & 6375 & 26 \\
\hline $\mathrm{H}\left(6^{\prime}\right)$ & 4531 & 612 & 6020 & 33 \\
\hline $\mathrm{H}\left(7^{\prime}\right)$ & 4207 & 3025 & 5466 & 35 \\
\hline $\mathrm{H}\left(9^{\prime}\right)$ & 6961 & 8758 & 6320 & 29 \\
\hline $\mathrm{H}\left(10^{\prime}\right)$ & 7390 & 7037 & 7045 & 29 \\
\hline $\mathrm{H}\left(11^{\prime}\right)$ & 6782 & 2577 & 6856 & 27 \\
\hline $\mathrm{H}\left(12^{\prime}\right)$ & 8227 & 1230 & 6556 & 29 \\
\hline$H\left(14^{\prime}\right)$ & 8408 & 7424 & 6019 & 30 \\
\hline$H\left(15^{\prime}\right)$ & 7807 & 2962 & 5830 & 30 \\
\hline $\mathrm{H}\left(17^{\prime}\right)$ & 9439 & 8899 & 6547 & 27 \\
\hline $\mathrm{H}\left(18^{\prime}\right)$ & 10651 & 9397 & 6880 & 30 \\
\hline $\mathrm{H}\left(19^{\prime}\right)$ & 11074 & 6549 & 7352 & 30 \\
\hline $\mathrm{H}\left(20^{\prime}\right)$ & 10276 & 3127 & 7482 & 29 \\
\hline $\mathrm{H}\left(21^{\prime}\right)$ & 9053 & 2617 & 7154 & 27 \\
\hline $\mathrm{H}(3)$ & 6098 & 2020 & 4419 & 30 \\
\hline $\mathrm{H}(5)$ & 6618 & -3585 & 3675 & 30 \\
\hline $\mathrm{H}(6)$ & 7805 & -4309 & 4031 & 37 \\
\hline $\mathrm{H}(7)$ & 8133 & -1928 & 4581 & 38 \\
\hline $\mathrm{H}(9)$ & 5329 & 3852 & 3766 & 27 \\
\hline $\mathrm{H}(10)$ & 4948 & 2218 & 3024 & 26 \\
\hline $\mathrm{H}(11)$ & 5578 & -2218 & 3196 & 28 \\
\hline $\mathrm{H}(12)$ & 4138 & -3732 & 3481 & 30 \\
\hline $\mathrm{H}(14)$ & 3883 & 2330 & 4045 & 28 \\
\hline $\mathrm{H}(15)$ & 4517 & -2099 & 4219 & 31 \\
\hline $\mathrm{H}(17)$ & 2843 & 3814 & 3486 & 32 \\
\hline $\mathrm{H}(18)$ & 1653 & 4194 & 3126 & 36 \\
\hline $\mathrm{H}(19)$ & 1316 & 1297 & 2639 & 36 \\
\hline $\mathrm{H}(20)$ & 2167 & -2045 & 2527 & 33 \\
\hline $\mathrm{H}(21)$ & 3340 & -2438 & 2895 & 29 \\
\hline
\end{tabular}


Symmetry transformations used to generate equivalent atoms:

$\# 1-\mathrm{x}+2, \mathrm{y},-\mathrm{z}+3 / 2 \quad \# 2-\mathrm{x}+1, \mathrm{y},-\mathrm{z}+1 / 2$

Table 4. Anisotropic displacement parameters $\left(\AA^{2} \times 10^{3}\right)$ for Baran589. The anisotropic displacement factor exponent takes the form: $-2 \pi^{2}\left[h^{2} a^{* 2} U^{11}+\ldots+2 h k a^{*} b^{*} U^{12}\right]$

\begin{tabular}{|c|c|c|c|c|c|c|}
\hline & $\mathrm{U}^{11}$ & $U^{22}$ & $\mathrm{U}^{33}$ & $\mathrm{U}^{23}$ & $\mathrm{U}^{13}$ & $\mathrm{U}^{12}$ \\
\hline $\mathrm{O}(1)$ & 14(4) & $15(2)$ & $25(4)$ & $-1(2)$ & 2(3) & 2(3) \\
\hline$C(1)$ & 14(4) & $15(2)$ & $25(4)$ & $-1(2)$ & 2(3) & 2(3) \\
\hline$C(2)$ & $24(2)$ & $33(2)$ & $15(2)$ & $8(2)$ & $5(1)$ & $4(2)$ \\
\hline$C(3)$ & $24(2)$ & $30(2)$ & $13(2)$ & $-4(2)$ & $2(1)$ & $-7(2)$ \\
\hline$C(4)$ & $22(2)$ & $10(2)$ & $14(2)$ & $-1(1)$ & $0(1)$ & $0(1)$ \\
\hline$C(5)$ & $23(2)$ & $16(2)$ & $11(2)$ & $-1(1)$ & $2(1)$ & $-2(1)$ \\
\hline$C(6)$ & $31(2)$ & $23(2)$ & $22(2)$ & $2(2)$ & $9(2)$ & $1(2)$ \\
\hline$C(7)$ & $34(2)$ & $41(2)$ & $20(2)$ & $0(2)$ & $9(2)$ & $-12(2)$ \\
\hline $\mathrm{C}(8)$ & $25(2)$ & $53(3)$ & $18(2)$ & $-10(2)$ & $7(2)$ & $-5(2)$ \\
\hline$C(9)$ & $26(2)$ & $36(2)$ & $27(2)$ & $-10(2)$ & $6(2)$ & $0(2)$ \\
\hline$C(10)$ & $25(2)$ & $27(2)$ & $17(2)$ & $2(2)$ & $4(2)$ & $1(1)$ \\
\hline $\mathrm{O}(2)$ & $37(5)$ & $19(2)$ & $32(2)$ & $-3(1)$ & $7(4)$ & $-2(4)$ \\
\hline$C(11)$ & $37(5)$ & $19(2)$ & $32(2)$ & $-3(1)$ & 7(4) & $-2(4)$ \\
\hline $\mathrm{C}(12)$ & $33(2)$ & $36(2)$ & $28(2)$ & $-3(2)$ & $8(2)$ & $9(2)$ \\
\hline $\mathrm{C}(13)$ & $33(2)$ & $31(2)$ & $28(2)$ & $4(2)$ & $11(2)$ & $-4(2)$ \\
\hline$C(14)$ & $25(2)$ & $21(2)$ & $17(2)$ & $2(1)$ & $6(2)$ & $3(1)$ \\
\hline$C(15)$ & $24(2)$ & $28(2)$ & $17(2)$ & $-1(2)$ & $7(1)$ & $-4(2)$ \\
\hline $\mathrm{C}(16)$ & $31(2)$ & $26(2)$ & $22(2)$ & $-1(2)$ & $8(2)$ & $1(2)$ \\
\hline$C(17)$ & $28(2)$ & $30(2)$ & $33(2)$ & $2(2)$ & $7(2)$ & $2(2)$ \\
\hline$C(18)$ & $29(2)$ & $41(2)$ & $22(2)$ & $4(2)$ & $7(2)$ & $-6(2)$ \\
\hline C(19) & $42(2)$ & $28(2)$ & $25(2)$ & $-7(2)$ & $10(2)$ & $-5(2)$ \\
\hline$C(20)$ & $30(2)$ & $31(2)$ & $28(2)$ & $-3(2)$ & $9(2)$ & $-3(2)$ \\
\hline
\end{tabular}


Table 5. Hydrogen coordinates ( $\left.\mathrm{x} 10^{4}\right)$ and isotropic displacement parameters $\left(\AA^{2} \mathrm{x} 10^{3}\right)$ for Baran589.

\begin{tabular}{|c|c|c|c|c|}
\hline & $\mathrm{x}$ & $\mathrm{y}$ & $\mathrm{z}$ & $\mathrm{U}(\mathrm{eq})$ \\
\hline $\mathrm{H}(1 \mathrm{~A})$ & 9378 & 7190 & 7435 & 23 \\
\hline $\mathrm{H}(1 \mathrm{~B})$ & 10058 & 6965 & 8547 & 23 \\
\hline $\mathrm{H}(2 \mathrm{~A})$ & 10160 & 1046 & 8835 & 30 \\
\hline $\mathrm{H}(2 \mathrm{~B})$ & 10517 & 3492 & 9332 & 30 \\
\hline $\mathrm{H}(3 \mathrm{~A})$ & 8686 & 3265 & 6536 & 28 \\
\hline $\mathrm{H}(3 \mathrm{~B})$ & 9085 & 916 & 7099 & 28 \\
\hline $\mathrm{H}(6)$ & 9171 & 920 & 9298 & 31 \\
\hline $\mathrm{H}(7)$ & 8275 & 1236 & 10130 & 38 \\
\hline $\mathrm{H}(8)$ & 7515 & 4523 & 9896 & 38 \\
\hline $\mathrm{H}(9)$ & 7654 & 7544 & 8839 & 37 \\
\hline $\mathrm{H}(10)$ & 8548 & 7276 & 8006 & 29 \\
\hline $\mathrm{H}(11 \mathrm{~A})$ & 5023 & 5007 & 3507 & 37 \\
\hline $\mathrm{H}(11 \mathrm{~B})$ & 5658 & 5094 & 2974 & 37 \\
\hline $\mathrm{H}(12 \mathrm{~A})$ & 5881 & -1159 & 2915 & 39 \\
\hline $\mathrm{H}(12 \mathrm{~B})$ & 6304 & 1156 & 2762 & 39 \\
\hline $\mathrm{H}(13 \mathrm{~A})$ & 4528 & 1657 & 3845 & 37 \\
\hline $\mathrm{H}(13 \mathrm{~B})$ & 4860 & -863 & 3754 & 37 \\
\hline $\mathrm{H}(16)$ & 6575 & -1088 & 4574 & 32 \\
\hline $\mathrm{H}(17)$ & 7403 & -981 & 6322 & 37 \\
\hline $\mathrm{H}(18)$ & 7405 & 2213 & 7383 & 37 \\
\hline H(19) & 6564 & 5278 & 6673 & 39 \\
\hline $\mathrm{H}(20)$ & 5723 & 5126 & 4928 & 36 \\
\hline
\end{tabular}




\section{References}

\section{For full description of Ref. 10 of the manuscript, see}

(10a) Chalmers, B. A.; Xing, H.; Houston, S.; Clark, C.; Ghassabian, S.; Kuo, A.; Cao, B.; Reitsma, A.; Murray, C.-E. P.; Stok, J. E.; Boyle, G. M.; Pierce, C. J.; Littler, S. W.; Winkler, D. A.; Bernhardt, P. V.; Pasay, C.; De Voss, J. J.; McCarthy, J.; Parsons, P. G.; Walter, G. H.; Smith, M. T.; Cooper, H. M.; Nilsson, S. K.; Tsanaktsidis, J.; Savage, G. P.; Williams, C. M., Angew. Chem. Int. Ed. 2016, 55, 35803585 .

(10b) Stepan, A. F.; Subramanyam, C.; Efremov, I. V.; Dutra, J. K.; O'Sullivan, T. J.; DiRico, K. J.; McDonald, W. S.; Won, A.; Dorff, P. H.; Nolan, C. E.; Becker, S. L.; Pustilnik, L. R.; Riddell, D. R.; Kauffman, G. W.; Kormos, B. L.; Zhang, L.; Lu, Y.; Capetta, S. H.; Green, M. E.; Karki, K.; Sibley, E.; Atchison, K. P.; Hallgren, A. J.; Oborski, C. E.; Robshaw, A. E.; Sneed, B.; O'Donnell, C. J., J. Med. Chem. 2012, 55, 3414-3424.

(1) Cornella, J.; Edwards, J. T.; Qin, T.; Kawamura, S.; Wang, J.; Pan, C. -M.; Gianatassio, R.; Schmidt, M.; Eastgate, M. D.; Baran, P. S. J. Am. Chem. Soc, 2016, 138, 2174.

(2) Qin, T.; Cornella, J.; Li, C.; Malins, L.; Edwards, J. T.; Kawamura, S.; Maxwell, B. D.; Eastgate, M. D.; Baran, P. S. Science, 2016, 352, 801.

(3) Wang, J.; Qin, T.; Chen, T. -G.; Wimmer, L.; Edwards, J. T.; Cornella, J.;Vokits, B.; Shaw, S. A.; Baran, P. S. Angew. Chem. Int. Ed. 2016, 55, 9676.

(4) Huihui, K. M. M.; Caputo, J. A.; Melchor, Z.; Olivares, A. M.; Spiewak, A. M.; Johnson, K. A.; DiBenedetto, T. A.; Kim, S.; Ackerman, L. K. G.; Weix, D. J., J. Am. Chem. Soc. 2016, 138, 5016-5019.

(5) Liu, D.; Li, Y.; Liu, C.; Lei, A.; Qi, X.; Lan, Y., Org. Lett. 2015, 17, 998 - 1001.

(6) Yasuda, S.; Yorimitsu, H.; Oshima, K., Bull. Chem. Soc. Jap. 2008, 81, 287 - 290.

(7) O'Sullivan, S.; Doni, E.; Tuttle, T.; Murphy, J. A., Angew. Chem. Int. Ed. 2014, 53, 474 - 478.

(8) Akiyama, R.; Kobayashi, S., J. Am. Chem. Soc. 2003, 125, 3412-3413.

(9) Benati, L.; Leardini, R.; Minozzi, M.; Nanni, D.; Spagnolo, P.; Strazzari, S.; Zanardi, G., Org. Lett. 2002, 4, 3079-3081.

(10) Grunewald, G. L.; Ye, Q., J. Org. Chem. 1988, 53, 4021 - 4026.

(11) Soule, J.-F.; Miyamura, H.; Kobayashi, S., J. Am. Chem. Soc. 2013, 135, 10602 - 10605.

(12) Mitchell, T. A.; Bode, J. W., J. Am. Chem. Soc. 2009, 131, 18057-18059.

(13) Shen, Z.-L.; Goh, K. K. K.; Yang, Y.-S.; Lai, Y.-C.; Wong, C. H. A.; Cheong, H.-L.; Loh, T.-P., Angew. Chem. Int. Ed. 2011, 50, 511 - 514.

(14) Lu, Z.; Fu, G. C., Angew. Chem. Int. Ed. 2010, 49, 6676.

(15) Maity, P. K.; Faisal, S.; Rolfe, A.; Stoianova, D.; Hanson, P. R., J. Org. Chem. 2015, 80, 99429950.

(16) Suau, R.; Garcia-Segura, R.; Sanchez-Sanchez, C.; Perez-Inestrosa, E.; Pedraza, A. M., Tetrahedron 2003, 59, 2913-2919.

(17) Dunsford, J. J.; Clark, E. R.; Ingleson, M. J., Angew. Chem. Int. Ed. 2015, 54, 5688 - 5692. 
(18) Birch, A. M.; Birtles, S.; Buckett, L. K.; Kemmitt, P. D.; Smith, G. J.; Smith, T. J. D.; Turnbull, A. V.; Wang, S. J. Y., J. Med. Chem. 2009, 52, 1558 - 1568.

(19) Kaszynski, P.; Friedli, A. C.; Michl, J., J. Am. Chem. Soc. 1992, 114, 601 - 620.

(20) Cheng, D.; Huang, D.; Shi, Y., Org. Biomol. Chem. 2013, 11, 5588 - 5591.

(21) Jackson, R. F. W.; Moore, R. J.; Dexter, C. S.; Elliott, J.; Mowbray, C. E., J. Org. Chem. 1998, 63, 7875-7884.

(22) Pouliot, M.-F.; Mahe, O.; Hamel, J.-D.; Desroches, J.; Paquin, J.-F., Org. Lett. 2012, 14, 54285431.

(23) Baker, B. A.; Boskovic, Z. V.; Lipshutz, B. H., Org. Lett. 2008, 10, 289-292.

(24) Della, E. W.; Head, N. J.; Mallon, P.; Walton, J. C., J. Am. Chem. Soc. 1992, 114, 10730 - 10738.

(25) Wlochal, J.; Davies, R. D. M.; Burton, J. Org. Lett. 2014, 16, 4094. 